\title{
RAYLEIGH FLOW OF TWO-PHASE NITROUS OXIDE AS A HYBRID ROCKET NOZZLE COOLANT
}

\author{
A Thesis \\ presented to \\ the Faculty of California Polytechnic State University, \\ San Luis Obispo

\begin{abstract}
In Partial Fulfillment
of the Requirements for the Degree

Master of Science in Mechanical Engineering
\end{abstract}

by

Lauren May Nelson

September 2009 
(C) 2009

Lauren May Nelson

ALL RIGHTS RESERVED 


\section{COMMITTEE MEMBERSHIP}

TITLE:

Rayleigh Flow of Two-Phase Nitrous Oxide as a Hybrid

Rocket Nozzle Coolant
AUTHOR:
Lauren May Nelson

DATE SUBMITTED: $\quad$ September 2009

COMMITTEE CHAIR: $\quad$ Dr. Patrick Lemieux, Associate Professor

COMMITTEE MEMBER: Dr. William Murray, Professor

COMMITTEE MEMBER: Dr. Joseph Mello, Professor 


\begin{abstract}
Rayleigh Flow of Two-Phase Nitrous Oxide as a Hybrid Rocket Nozzle Coolant Lauren May Nelson
\end{abstract}

The Mechanical Engineering Department at California Polytechnic State University in San Luis Obispo currently maintains a lab-scale hybrid rocket motor for which nitrous oxide is utilized as the oxidizer in the combustion system. Because of its availability, the same two-phase (gas and liquid) nitrous oxide that is used in the combustion system is also routed around the throat of the hybrid rocket's converging-diverging nozzle as a coolant. While this coolant system has proven effective empirically in previous tests, the physics behind the flow of the two-phase mixture is largely unexplained. This thesis provides a method for predicting some of its behavior by modeling it using the classic gas dynamics scenarios of Rayleigh and Fanno flows which refer to one-dimensional, compressible, inviscid flow in a constant area duct with heat addition and friction. The two-phase model produced utilizes a separated phase with interface exchange model for predicting whether or not dryout occurs. The Shah correlation is used to predict heat transfer coefficients in the nucleate boiling regime. The homogeneous flow model is utilized to predict pressure drop. It is proposed that a Dittus-Boelter based correlation much like that of Groeneveld be developed for modeling heat transfer coefficients upon the collection of sufficient data.

Data was collected from a series of tests on the hybrid rocket nozzle to validate this model. The tests were first run for the simplified case of an ideal gas (helium) coolant to 
verify the experimental setup and promote confidence in subsequent two-phase experimental results. The results of these tests showed good agreement with a combined Rayleigh-Fanno model with a few exceptions including: (1) reduced experimental gas pressure and temperature in the annulus entrance and exit regions compared to the model and (2) reduced experimentally measured copper temperatures uniformly through the annulus. These discrepancies are likely explained by the geometry of the flowpath and location of the copper thermocouples respectively. Next, a series of two-phase cooled experiments were run. Similar trends were seen to the helium experiment with regards to entrance and exit regions. The two-phase Rayleigh homogeneous flow model underpredicted pressure drop presumably due to the inviscid assumption. Ambiguity was observed in the fluid temperature measurements but the trend seemed to suggest that mild thermal non-equilibrium existed. In both cases, the dryout model predicted that mist flow (a post-CHF regime) occurred over most of the annulus.

Several modifications should be implemented in future endeavors. These include: (1) collecting more data to produce a heat transfer coefficient correlation specific to the nitrous oxide system of interest, (2) accounting for thermal non-equilibrium, (3) accounting for entrance and exit effects, and (4) developing a two-phase Fanno model. 


\section{ACKNOWLEDGMENTS}

I would like to thank Dr's Lemieux, Murray and Mello for their guidance on this project. Also, special thanks to Terry Cooke and Jim Gerhardt for their time devoted to setting up and running the experiments. Finally, thanks to Peter Brennen for his work preceding this thesis. 


\section{TABLE OF CONTENTS}

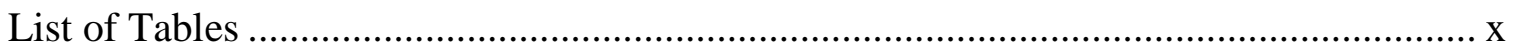

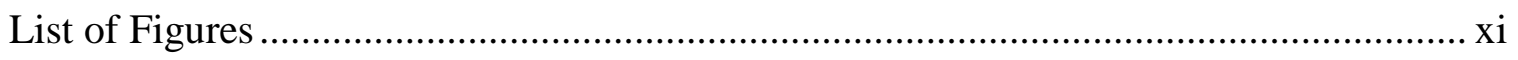

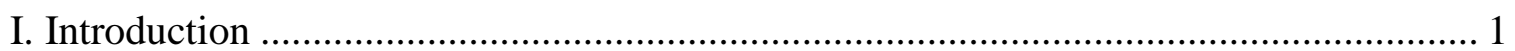

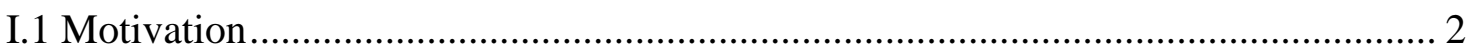

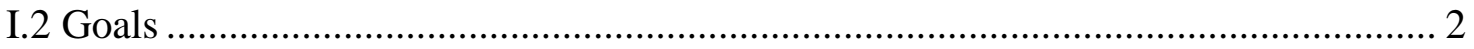

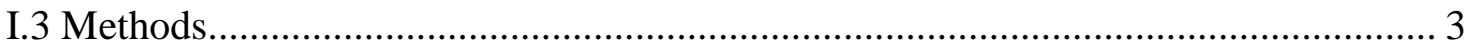

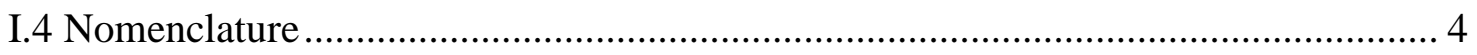

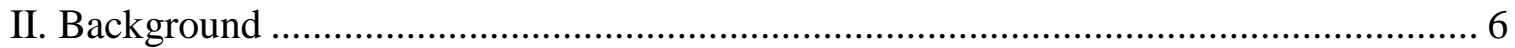

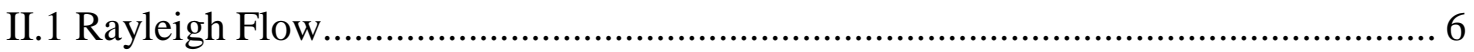

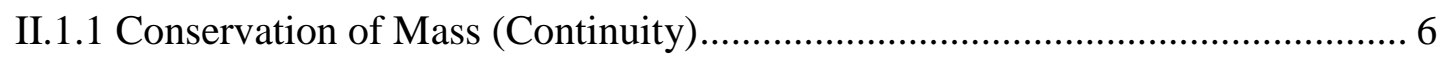

II.1.2 Conservation of Momentum and the Rayleigh Line ........................................ 7

II.1.3 Conservation of Energy ...................................................................... 9

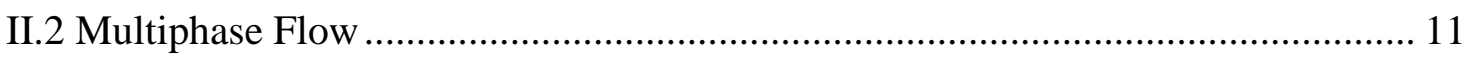

II.2.1 Two-Phase Governing Equations ............................................................ 13

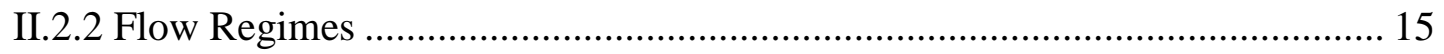

II.2.3 Critical Heat Flux and Dryout....................................................................... 17

II.2.4 Thermal Equilibrium................................................................................. 21

II.2.5 Pre-Existing Two-phase Heat Transfer Models................................................ 22

II.3 Previous Hybrid Rocket Testing at Cal Poly ..................................................... 25

II.3.1 Hot Side Heat Transfer Characteristics............................................................ 33

II.3.2 Biot Number Analysis.............................................................................. 42

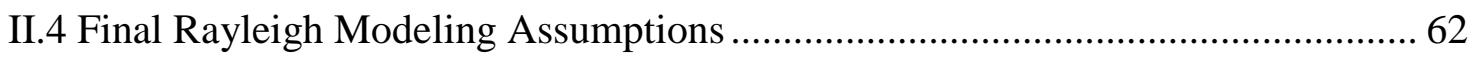

III: The Rayleigh Experiment Design and Preparation....................................................... 64

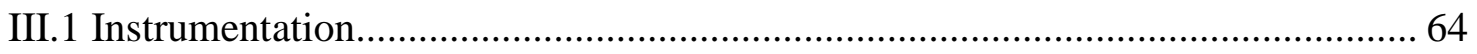

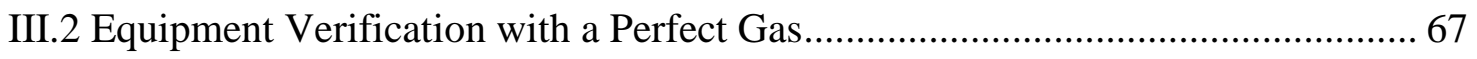

III.2.1 Experimental Design .............................................................................. 68

III.2.2 Helium Perfect Gas Modeling .................................................................... 75

III.2.3 Helium Modeling Results ......................................................................... 83 


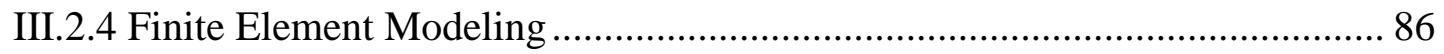

III.2.5 Ideal Gas Experiment Setup ……………………....................................... 90

III.2.6 Fanno Flow Model..................................................................................... 98

III.2.7 Helium Experiments ................................................................................. 99

III.2.8 Ideal Gas Results Discussion.................................................................... 113

III.2.9 Ideal Gas Conclusions ......................................................................... 118

IV: Two-Phase Coolant Modeling and Experiments ....................................................... 119

IV.1 Preliminary Modeling and Problem Setup...................................................... 120

IV.1.1 Fluid Properties ........................................................................................ 121

IV.1.2 System Schematic and Description .......................................................... 123

IV.2 Homogeneous Flow Model....................................................................... 125

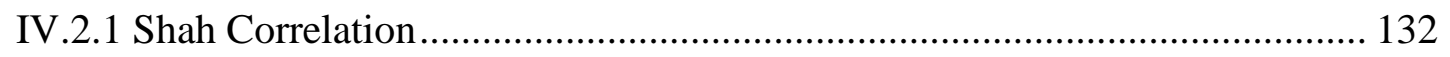

IV.2.2 Dryout Prediction ................................................................................... 137

IV.2.3 Post-Superheat Modeling ........................................................................... 138

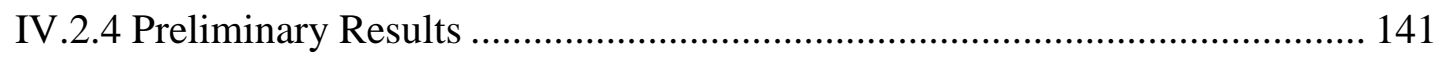

IV.3 Revellin-Thome Dryout Model.................................................................. 145

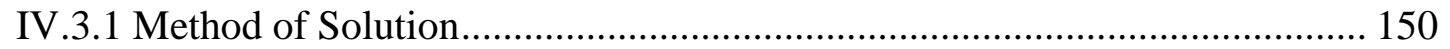

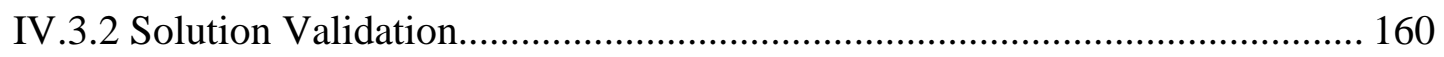

IV.3.3 Application to Nitrous Oxide ................................................................... 164

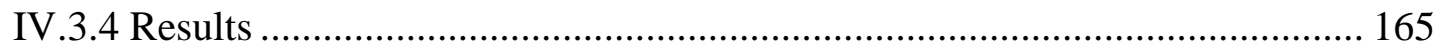

IV.4 Final Two-Phase Modeling ......................................................................... 169

IV.4.1 Groeneveld Correlation .......................................................................... 172

IV.4.2 Final Two-Phase Model Results............................................................ 173

IV.5 Two-Phase Nitrous Oxide Experiments........................................................... 175

IV.5.1 Preliminary Analysis .............................................................................. 176

IV.5.2 First Nitrous Oxide Cooled Experiment Results........................................... 177

IV.5.3 Second Nitrous Oxide Cooled Experiment Results ..................................... 184

IV.6 Two-Phase Results Discussion ..................................................................... 189

IV.6.1 Input Parameter Selection ....................................................................... 190

IV.6.2 Coolant Temperature Discrepancies ......................................................... 192

IV.7 Two-Phase Modeling Conclusions .................................................................. 192 
V. Conclusions and Recommendations 


\section{LIST OF TABLES}

Table 1. Experimental cold side heat transfer coefficients in BTU/in ${ }^{2}$ secR...................60

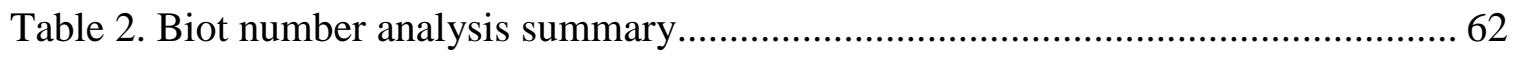

Table 3. Perfect gas cooling capacity analysis and physical properties summary........... 74

Table 4. Solenoid choking experimental and predicted mass flow rates ....................... 95

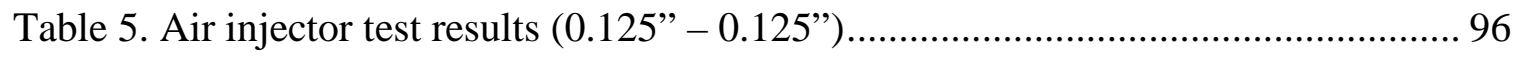

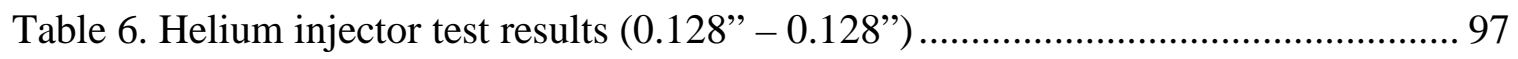

Table 7. Modeling technique as a function of flow regime ....................................... 120

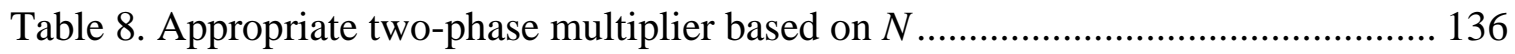

Table 9. Nitrous oxide Rayleigh experiment test conditions ................................... 176 


\section{LIST OF FIGURES}

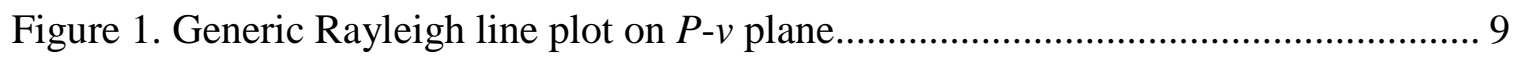

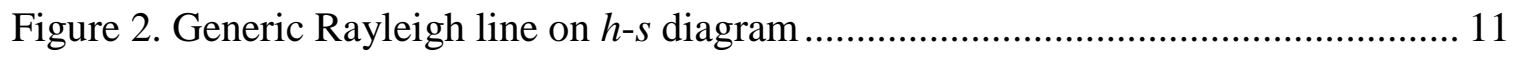

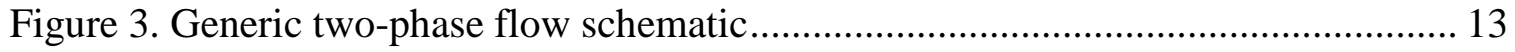

Figure 4. Typical flow regimes encountered in horizontal flow (4) ............................ 16

Figure 5. Typical vertical two-phase flow regimes (4) ......................................... 16

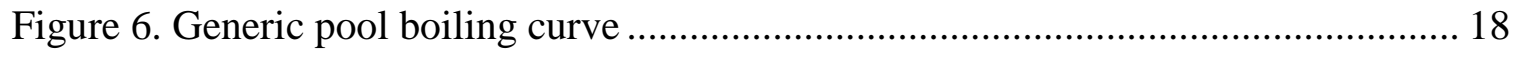

Figure 7. Flow boiling curve at constant mass flux and pressure (5) .......................... 19

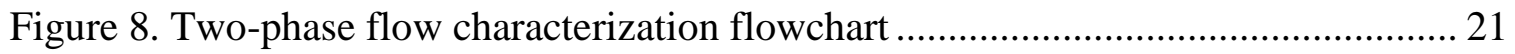

Figure 9. Top down schematic of two flow-paths nozzle geometry ............................ 26

Figure 10. Top down view of the original nozzle (left) and the Rayleigh nozzle (right). Red lines represent measurement stations..................................26

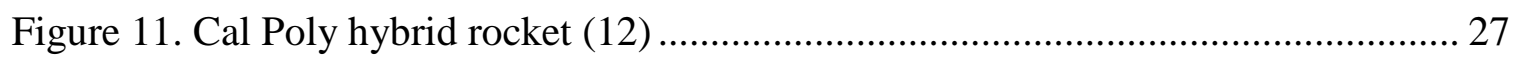

Figure 12. Cal Poly cooled converging-diverging nozzle (12) ................................... 27

Figure 13. Top-down image of the two flow-paths nozzle and instrumentation (12)..... 28

Figure 14. Rayleigh nozzle copper throat insert.................................................. 29

Figure 15. Sample data output from Cal Poly hybrid rocket tests ................................ 30

Figure 16. Top down schematic of the two flow-paths nozzle................................... 31

Figure 17. Copper temperatures through annulus in previous two-phase cooled tests..... 32

Figure 18. Fluid temperatures through annulus in previous two-phase cooled tests ........ 32

Figure 19. Bartz heat transfer coefficient distribution through the converging-

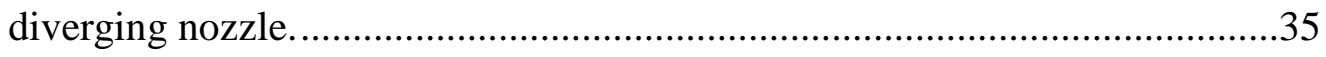

Figure 20. Control volume for radiation heat transfer in nozzle throat .......................... 37 
Figure 21. Biot analyses necessary to fully characterize copper ring ........................... 44

Figure 22. One-dimensional modeling of copper ring in radial Biot analyses (12) ........ 46

Figure 23. Low radial Biot number assumption (12) .............................................. 46

Figure 24. Schematic of high Biot number scenario (12) ......................................... 49

Figure 25. Radial hot side Biot numbers ......................................................... 51

Figure 26. Radial cold side Biot numbers ........................................................ 51

Figure 27. Two flow-paths nozzle top down schematic ......................................... 52

Figure 28. Circumferential Biot number control volume ........................................ 55

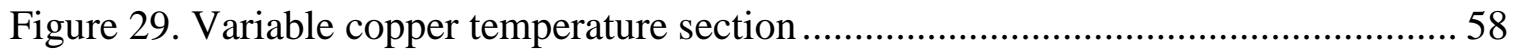

Figure 30. Experimental cold side heat transfer coefficients................................... 59

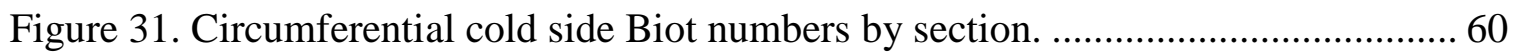

Figure 32. Circumferential hot side Biot numbers by section.................................. 61

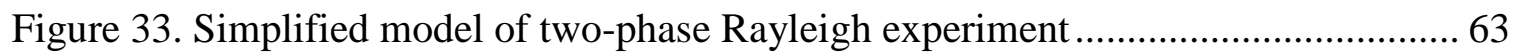

Figure 34. Modifications from the two flow-paths (left) to the Rayleigh nozzles

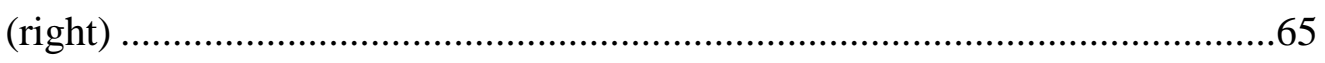

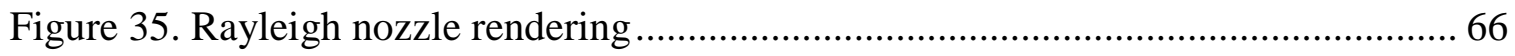

Figure 36. Location of coolant pressure transducer and coolant/copper thermocouples.. 67

Figure 37. Previous firing conditions for all recorded Plexiglas ${ }^{\circledR} / \mathrm{N}_{2} \mathrm{O}$ tests at Cal

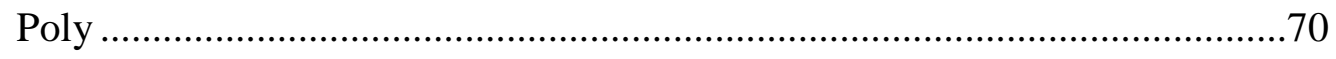

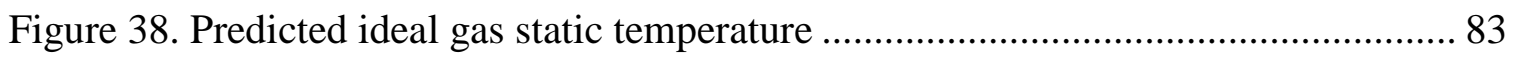

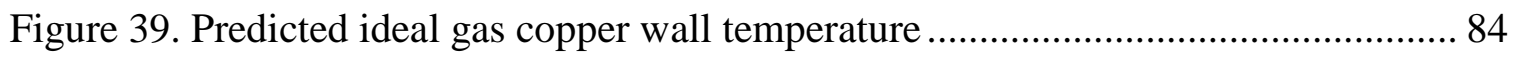

Figure 40. Predicted ideal gas static pressure ..................................................... 84

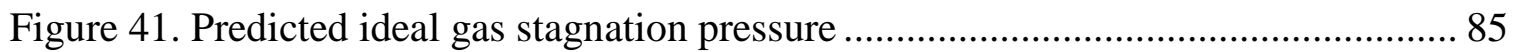




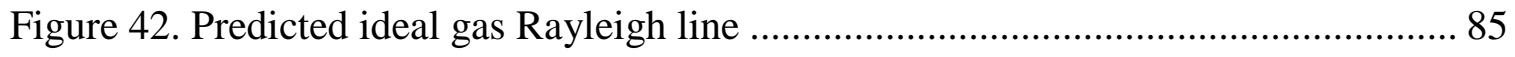

Figure 43. Predicted ideal gas Mach number.......................................................... 86

Figure 44. Temperature contour plot in degrees Fahrenheit of the copper insert............ 87

Figure 45. Comparing FE and analytical modeling predicted copper temperatures......... 88

Figure 46. Simplified helium coolant system schematic ....................................... 90

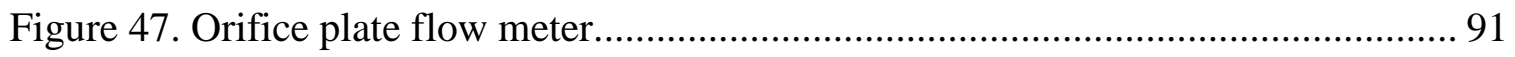

Figure 48. Correcting for ground-looping in gas and copper temperature measurements ............................................................................... 100

Figure 49. Helium test 1: transient pressure profiles in the steady state region

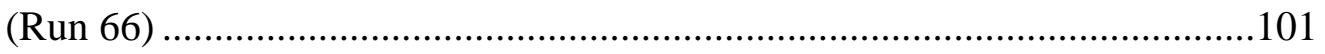

Figure 50. Helium test 1: transient copper temperature profiles in the steady state

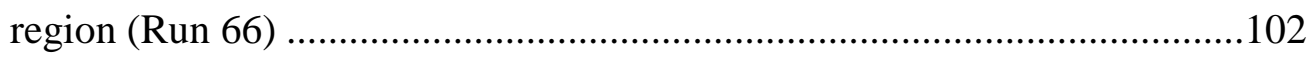

Figure 51. Helium test 1: transient gas temperature profiles in the steady state region (Run 66)

Figure 52. Helium test 1: transient mass flow rate profile in the steady state region..... 103

Figure 53. Helium test 1: predicted and measured average steady-state pressures ........ 104

Figure 54. Helium test 2: predicted and measured average gas temperatures ............... 105

Figure 55. Helium test 1: predicted and measured steady state wall temperatures ........ 105

Figure 56. Helium test 1: predicted and measured copper wall temperature increase ... 106

Figure 57. Helium test 1: predicted and experimental coolant heat transfer coefficients

Figure 58. Helium test 2: transient pressure profiles in the steady state region (Run 67) .108 
Figure 59. Helium test 2: transient copper temperature profiles in the steady state region (Run 67) .108

Figure 60. Helium test 2: transient gas temperature profiles in the steady state region (Run 67) 109

Figure 61. Helium test 2: mass flow rate profile in the steady state region 109

Figure 62. Helium test 2: predicted and measured average steady-state pressures ........ 110

Figure 63. Helium test 2: predicted and measured average gas temperatures 111

Figure 64. Helium test 2: predicted and measured steady state wall temperatures 111

Figure 65. Helium test 2: predicted and measured copper wall temperature increase ... 112 Figure 66. Helium test 2: predicted and experimental coolant heat transfer coefficients

Figure 67. Nitrous oxide cooled hot fire results (test 1 on the left, test 2 on the right) .. 114

Figure 68. Radial copper temperature distribution in degrees Fahrenheit (17) 115

Figure 69. Helium test 1: experimentally measured copper temperature 117

Figure 70. Helium test 2: experimentally measured copper temperature 117

Figure 71. Simplified model of two-phase Rayleigh flow 120

Figure 72. Similarity of specific heats of $\mathrm{CO}_{2}$ and $\mathrm{N}_{2} \mathrm{O}$

Figure 73. Percent difference between specific heats of $\mathrm{CO}_{2}$ and $\mathrm{N}_{2} \mathrm{O}$ 123

Figure 74. Coolant system schematic 124

Figure 75. Isenthalpic throttling process across inlet injector 124

Figure 76. Annulus cross-section with (right) and without (left) 1-D flow assumption. .126

Figure 77. Vapor quality as predicted by the homogeneous flow model for SOC 142 
Figure 78. Fluid temperature as predicted by homogeneous flow model for SOC 143

Figure 79. Copper wall temperature as predicted by homogeneous model for SOC ..... 143

Figure 80. Fluid pressure as predicted by the homogeneous model for SOC................ 144

Figure 81. Predicted coolant heat transfer coefficient for SOC .................................. 144

Figure 82. Annular separated flow model control volume (10)................................ 146

Figure 83. Interfacial wave dryout in annular flow regime ................................... 150

Figure 84. Discretized channel for numerical solution of separated flow ODEs ........... 150

Figure 85. Liquid film profile predicted Revellin and Thome (10)............................ 161

Figure 86. Liquid film profile predicted by Taylor series approximation .................... 161

Figure 87. Liquid and vapor velocities predicted by Revellin and Thome (10) ............ 162

Figure 88. Liquid and vapor velocities predicted by Taylor series approximation ........ 162

Figure 89. Liquid and vapor pressures predicted by Revellin and Thome (10) ............ 163

Figure 90. Liquid and vapor pressures predicted by Taylor series approximation......... 163

Figure 91. Physical two-phase injector (top) and two-phase injector model (bottom)... 165

Figure 92. Predicted Static fluid pressures through injector and annulus until dryout... 166

Figure 93. Predicted quality through injector and annulus until dryout ....................... 167

Figure 94. Mist flow (in contrast to previously discussed flow regimes) (23) .............. 168

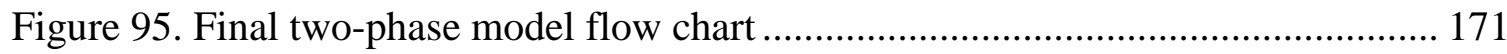

Figure 96. Mixture temperatures as predicted by the two-phase model ...................... 174

Figure 97. Mixture pressure as predicted by the two-phase model ............................ 174

Figure 98. Quality as predicted by the two-phase model........................................ 175

Figure 99. Nitrous oxide test 1: transient copper temperatures (Run 68) ...................... 177

Figure 100. Nitrous oxide test 1: transient annulus pressures (Run 68) ........................ 178 
Figure 101. Nitrous oxide test 1: transient fluid temperatures (Run 68) 178

Figure 102. Nitrous oxide test 1: transient scale and flow meter mass flow rates

(Run 68)

Figure 103. Thermocouple 3 behaves as expected without a heat load 180

Figure 104. $\mathrm{N}_{2} \mathrm{O}$ test 1: steady state fluid pressures 181

Figure $105 . \mathrm{N}_{2} \mathrm{O}$ test 1: steady state fluid temperature. 182

Figure $106 . \mathrm{N}_{2} \mathrm{O}$ test 1 : steady state copper temperatures 182

Figure 107. $\mathrm{N}_{2} \mathrm{O}$ test 1: measured and predicted heat transfer coefficients. 183

Figure 108. $\mathrm{N}_{2} \mathrm{O}$ test 1 : thermodynamic quality through the annulus 183

Figure 109. $\mathrm{N}_{2} \mathrm{O}$ test 2: transient coolant pressures in steady state region (Run 69)..... 184

Figure 110. $\mathrm{N}_{2} \mathrm{O}$ test 2: transient copper temperatures in steady state region (Run 69) 185

Figure 111. $\mathrm{N}_{2} \mathrm{O}$ test 2: transient coolant temperatures in steady state region (Run 69) 185

Figure 112. $\mathrm{N}_{2} \mathrm{O}$ test 2: transient mass flow rate in steady state region (Run 69) ......... 186

Figure $113 . \mathrm{N}_{2} \mathrm{O}$ test 2: steady state coolant pressures 187

Figure $114 . \mathrm{N}_{2} \mathrm{O}$ test 2 : steady state coolant temperatures 187

Figure $115 . \mathrm{N}_{2} \mathrm{O}$ test 2 : steady state copper temperatures 188

Figure 116. $\mathrm{N}_{2} \mathrm{O}$ test 2: measured and predicted coolant heat transfer coefficients ..... 188

Figure $117 . \mathrm{N}_{2} \mathrm{O}$ test 2 : thermodynamic quality 189

Figure 118. $\mathrm{N}_{2} \mathrm{O}$ test 1: coolant pressures with second measurement as input. 191

Figure 119. $\mathrm{N}_{2} \mathrm{O}$ test 2: coolant pressures with second measurement as input. 191 


\section{INTRODUCTION}

The Mechanical Engineering Department at California Polytechnic State University in San Luis Obispo (Cal Poly) currently maintains a lab-scale hybrid rocket motor and test stand. One goal of maintaining this equipment is to investigate the feasibility of single stage to orbit (SSTO) air vehicles. Of primary interest is the aerospike nozzle which is a popular choice for SSTOs as its geometry allows it to adjust to atmospheric pressure, preventing some of the thrust loss seen by traditional converging-diverging ("con-di") nozzles at high altitudes. Unfortunately, aerospike nozzles are known to be especially susceptible to overheating and therefore require advanced cooling systems to prevent heat damage and prolong their lifespan. The Cal Poly hybrid rocket motor is currently equipped with a con-di nozzle around which multiphase (gas-liquid) nitrous oxide (" $\mathrm{N}_{2} \mathrm{O}$ ") is routed as a coolant. While this cooling system has proven successful empirically in previous tests at Cal Poly, it has not been studied analytically and its behavior is not well understood. The purpose of this thesis is to produce one or several models that are capable of predicting or explaining the behavior of the two-phase nitrous oxide as it flows around the throat of the con-di nozzle. Sufficient understanding of this coolant system will aid in the overall goal of developing a SSTO air vehicle as the model developed herein may be extended to an aerospike nozzle.

Typically, a hybrid rocket is defined as one in which the oxidizer and fuel are initially in different phases. In the case of the Cal Poly hybrid rocket, the fuel is solid Plexiglas ${ }^{\circledR}$ and its oxidizer is liquid nitrous oxide $\left(\mathrm{N}_{2} \mathrm{O}\right)$. Currently, the rocket and test stand are instrumented to monitor pressure and temperature at crucial locations as well as thrust 
produced by the motor. Nitrous oxide is a prime choice for coolant in this case because: (1) it undergoes a phase change while flowing around the nozzle throat, offering a larger cooling capacity than a single phase convective coolant and (2) it is readily available as it is also used as the oxidizer in the propulsion system.

\section{I.1 MOTIVATION}

At extreme conditions, specifically high temperatures, nitrous oxide has been known to decompose exothermically (1). During this decomposition, each mole of nitrous oxide dissociates into one mole of nitrogen and one half mole of oxygen. Because of its negative heat of formation, this decomposition is also accompanied by a release of energy which could potentially trigger additional decomposition. In this way, even the slightest amount of decomposition may result in a cascade of heating and possible explosion.

One motivation for this project is this possibility of exothermic decomposition of the coolant which would be detrimental to the hybrid rocket and pose safety risks as it would lead to heating of the nozzle rather than cooling. The combination of the potential of nitrous oxide to decompose exothermically and the aerospike to plug and catastrophically self-destruct if overheated makes it of primary importance that the nitrous oxide cooling system is well understood prior to utilizing it with the aerospike nozzle.

\section{I.2 GOALS}

Two distinct paths will be taken in an effort to characterize the nitrous oxide coolant system. First, one or several predictive models to describe the nitrous oxide as it flows 
through the coolant path will be formulated. Second, experimental data will be gathered to validate this model. The two goals for the model developed are: (1) approximating the convective heat transfer coefficients and pressure drop for the two-phase nitrous oxide as it flows through the coolant annulus and (2) predicting the conditions at which dryout occurs. This concept will be discussed in more detail in subsequent sections

\section{I.3 METHODS}

Many empirical and analytical two-phase models currently exist. The modeling side of this thesis will include evaluation of these pre-existing models and application of one or several that are deemed appropriate. In order to validate the predictive models, relevant experimental data will be collected. While some data has already been collected at Cal Poly in previous tests, it was not tailored to fit the needs of this study and is not comprehensive enough to validate a model of this nature. Instead, the necessary data will be gathered from a series of new tests using a cooling system with slightly modified geometry from those used in the past. The new nozzle includes a single coolant path running around the nozzle throat with constant heat flux being added from the combustion products. This situation closely resembles the classic gas dynamics problem known as Rayleigh flow in which fluid flows in a constant area duct with constant heat flux and will therefore be termed the "Rayleigh experiment." Likewise, the nozzle on which these tests are run is referred to as the "Rayleigh nozzle." 


\section{I.4 NOMENCLATURE}

A list of variable definitions will be a useful reference for the remainder of this document. Unfortunately, a finite number of symbols exist and it was impossible to avoid using some variables to represent more than one physical characteristic.

\begin{tabular}{|c|c|c|c|}
\hline$A$ & Area & $L_{e}$ & Effective beam length \\
\hline$B i$ & Biot number & $M$ & Mach number \\
\hline Bo & Boiling number & $\dot{m}$ & Mass flow rate \\
\hline$c$ & Speed of sound & $\mathrm{Nu}$ & Nusselt number \\
\hline$C_{d}$ & Discharge coefficient & $P$ & Pressure \\
\hline$C_{f}$ & Friction factor & $P_{c}$ & Chamber pressure \\
\hline Co & Convection number & $\operatorname{Pr}$ & Prandtl number \\
\hline$C_{p}$ & Specific heat & $Q$ & Heat transfer \\
\hline$D$ & Diameter & $q$ & Heat transfer rate \\
\hline$D_{H}$ & Hydraulic diameter & $R$ & Specific gas constant \\
\hline$f$ & friction factor & $R$ & Radius \\
\hline $\mathrm{Fr}$ & Froude number & $r_{c}$ & Radius of curvature \\
\hline $\mathrm{g}$ & Gravitational constant & $R e$ & Reynolds number \\
\hline$G$ & Mass flux & $s$ & Specific entropy \\
\hline$h$ & Specific enthalpy & $s_{\text {arc }}$ & Arc length \\
\hline$h$ & Heat transfer coefficient & $S t$ & Stanton number \\
\hline$k$ & Thermal conductivity & $T$ & Temperature \\
\hline$L_{c}$ & Characteristic length & $u$ & Velocity \\
\hline
\end{tabular}




\begin{tabular}{|c|c|c|c|}
\hline \multirow{2}{*}{$\begin{array}{l}V \\
x\end{array}$} & \multirow{2}{*}{$\begin{array}{l}\text { Volume } \\
\text { Quality }\end{array}$} & \multicolumn{2}{|c|}{ Subscripts/Superscripts } \\
\hline & & ave & Average \\
\hline \multirow[t]{2}{*}{$\mathrm{z}$} & Axial location in annulus & cold & Cold side condition \\
\hline & & $c s$ & Cross-sectional \\
\hline \multicolumn{2}{|l|}{ Greek } & $\mathrm{Cu}$ & Copper \\
\hline$\alpha$ & Void fraction & do & Dryout \\
\hline$\alpha$ & Absorptivity & $g$ & Gas \\
\hline$\gamma$ & Ratio of specific heats & hot & Hot side condition \\
\hline$\varepsilon$ & Emissivity & $i$ & interfacial \\
\hline$\delta$ & Liquid film thickness & $l$ & Liquid \\
\hline$\theta$ & Angle & $L$ & Liquid \\
\hline$\mu$ & Dynamic viscosity & $L W$ & Liquid-wall \\
\hline$v$ & Kinematic viscosity & $L V$ & Liquid-vapor \\
\hline$\rho$ & Density & $\max$ & Maximum \\
\hline$\sigma$ & Boltzmann constant & $\min$ & Minimum \\
\hline$\tau$ & Shear stress & $n c$ & Neighboring cold \\
\hline \multirow[t]{6}{*}{$\varphi$} & Two-phase multiplier & $n h$ & Neighboring hot \\
\hline & & $T P$ & Two-phase \\
\hline & & V & Vapor \\
\hline & & $v$ & Vapor \\
\hline & & $*$ & Sonic \\
\hline & & 0 & Stagnation \\
\hline
\end{tabular}




\section{BACKGROUND}

A proper understanding of the concepts, theories, and previous experiments relevant to this project is vital to understanding the subsequent modeling and experimentation. The information discussed in this chapter includes basic and intermediate explanations of relevant topics such as: Rayleigh flow, two-phase flow, and previous experiments run at the Cal Poly.

\section{II.1 RAYLEIGH FLOW}

In the study of gas dynamics, steady, one-dimensional, diabatic, inviscid flow in a constant area duct is often referred to as Rayleigh flow. Despite the complexities introduced by its two-phase nature, the nitrous oxide system of interest closely resembles Rayleigh flow in many ways. A basic understanding of the equations governing this type of flow will therefore be beneficial in subsequent models. Applying the above conditions to the general forms of the three conservation laws gives the following set of governing equations for Rayleigh flow.

\section{II.1.1 Conservation of Mass (Continuity)}

The general form of the continuity equation for one-dimensional, steady flow in a constant area duct is: 


$$
\rho u=G
$$

where $u$ is the velocity, $\rho$ is the density and $G$ is a constant often referred to as the mass flux. $G$ is also equal to the ratio of the mass flow rate $\dot{m}$ to the cross sectional area of the flow path $A$, or

$$
G=\frac{\dot{m}}{A}
$$

\section{II.1.2 Conservation of Momentum and the Rayleigh Line}

For one-dimensional, steady flow in a constant area duct with negligible body and shear forces, the law of conservation of momentum in differential form is given by (2):

$$
d P+\rho u d u=0
$$

where $P$ is the static pressure. Note that in the case of incompressible flow, letting density remain constant and integrating the above expression leads to a common form of the Bernoulli equation

$$
\Delta P=\frac{1}{2} \rho u^{2}
$$

However, in the study of compressible flows, this constant density assumption is not valid. Instead, substituting the continuity equation into the above expression gives: 


$$
d P+G d u=0
$$

Integrating equation (1.5) above between two arbitrary states (1) and (2) gives:

$$
P_{1}+G u_{1}=P_{2}+G u_{2}=\text { constant }
$$

Solving for the pressure drop between states (1) and (2) yields:

$$
P_{1}-P_{2}=G\left(u_{2}-u_{1}\right)
$$

Finally, substituting the continuity equation into the above expression for $u_{1}$ and $u_{2}$ gives:

$$
P_{1}-P_{2}=G^{2}\left[\frac{1}{\rho_{2}}-\frac{1}{\rho_{1}}\right]
$$

It is often convenient to write this in terms of the specific volume which is simply the inverse of density:

$$
P_{1}-P_{2}=G^{2}\left[v_{2}-v_{1}\right]
$$

Equation (1.9) above is often called the Rayleigh line equation. Because $G$ remains constant, when plotted on the $P-v$ plane, it represents a straight line with a slope of $-G^{2}$. This indicates that a change in pressure between arbitrary states (1) and (2) is linear with 
respect to specific volume. Figure 1 below plots a generic Rayleigh line for a given $G$ value with two lines of constant enthalpy.

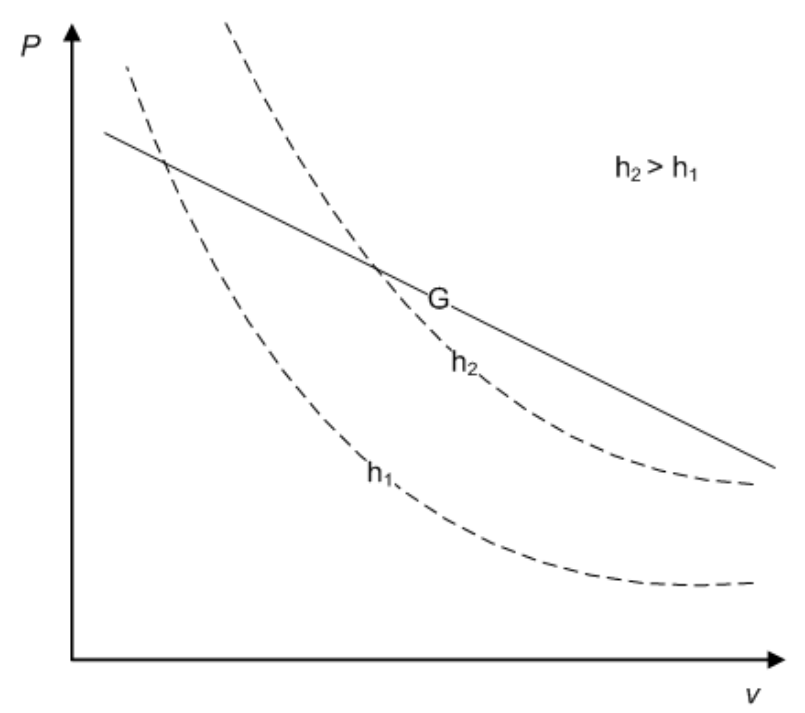

Figure 1. Generic Rayleigh line plot on $P-v$ plane

\section{II.1.3 Conservation of Energy}

For one-dimensional, steady flow with no external work, changes in potential energy or heat generation, the energy equation simplifies to

$$
d h+d\left(\frac{u^{2}}{2}\right)=\delta Q
$$

where $h$ is the static enthalpy and $Q$ is the heat transfer. Integrating between arbitrary states (1) and (2) gives 


$$
h_{2}-h_{1}+\frac{1}{2}\left(u_{2}^{2}-u_{1}^{2}\right)=Q_{1-2}
$$

where $Q_{1-2}$ is the heat transfer between states (1) and (2). This equation provides the link between the thermal and hydrodynamic behavior of the system. Note that for a process in which heat is added to the system, enthalpy will increase. Referring to Figure 1, this indicates that for a heat addition process, the pressure will decrease and the specific volume will increase.

Another useful tool in Rayleigh flow is the $h-s$ diagram of which a generic version is shown in Figure 2 below. This is also derived from the Rayleigh line equation and is plotted for a single $G$ value. By the second law of thermodynamics, it can be shown that at point $*$, the Mach number of the system will be equal to unity and at this point the system is termed "choked." If this is to occur in a given system, it must occur at the point of minimum cross-sectional area or the end of the duct if it in the case of constant area. The top branch of the curve in Figure 2 represents the locus of possible states for a fluid that enters the duct subsonically for a given mass flux while the lower branch gives those for a fluid that enters the duct supersonically. In both cases, the system approaches a Mach number of unity. In the subsonic case, the fluid is accelerating whereas for the supersonic one, the flow is decelerating. 


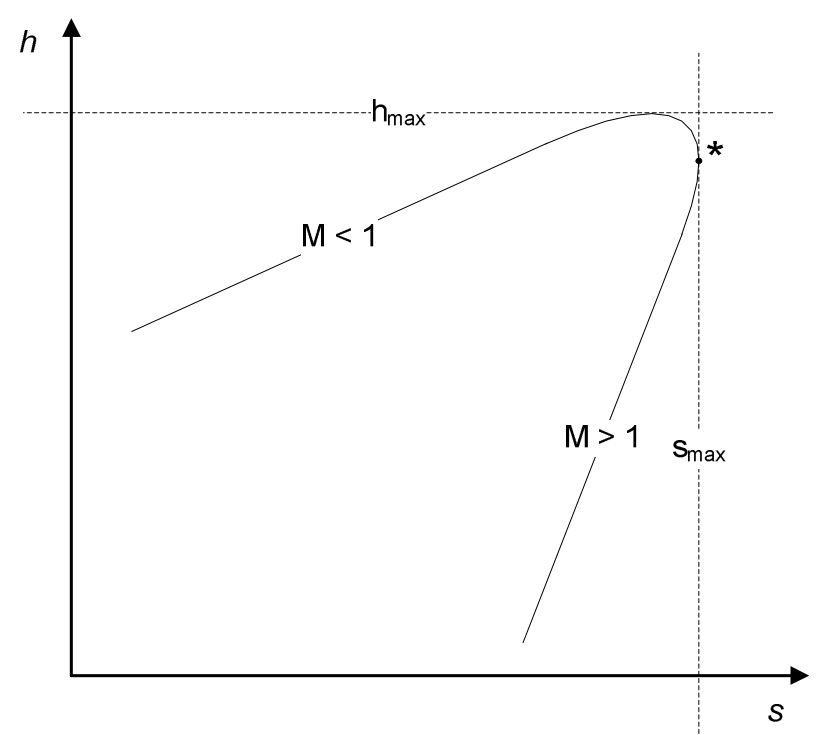

Figure 2. Generic Rayleigh line on $h-s$ diagram

The above equations and discussion were derived specifically for one-dimensional, inviscid, steady flow with heat addition in a constant area duct. Whether or not the gas is a perfect one was not specified which indicates that the equations may be applied to any fluid that meets the above criteria. Accordingly, two-phase flows could be modeled by the above equations. However, the assumption of one-dimensional flow requires that the fluid properties be averaged across any given cross section and any information about the individual liquid and gas phases is lost. In essence, this is what the two-phase homogeneous flow model assumes. This model is discussed in subsequent sections.

\section{II.2 MULTIPHASE FLOW}

The term multiphase flow encompasses a wide range of physical phenomena. It can refer to the flow of two different fluids, a fluid and a solid, a single saturated fluid or a combination of any of these. It is evident with such a large scope of topics, that there is a 
significant amount of background information necessary to fully understand multiphase flows. The purpose of this section is not to discuss every detail of multiphase flows but rather to refer only to those that are directly or indirectly relevant to this thesis. That is, only topics pertaining to the internal flow of a single saturated fluid will be discussed. Of particular interest are subjects that will be useful in modeling the thermal or hydrodynamic behavior (i.e. heat transfer coefficients or pressure drop) of the system.

The field of multiphase flow known as "two-phase flow" refers to a single saturated fluid. While two-phase flows are of interest to almost all engineering industries, the nuclear power industry has a particular investment in the topic. Two-phase flows in this industry are often used to cool large nuclear reactors, an application with especially severe consequences in the case of cooling system failure. Typically, the fluid of interest is water and appropriately much of the available data and empirical correlations for twophase flows are applicable only to steam-water systems. Other areas in which two-phase flows are of interest are the chemical engineering industry, and atmospheric science (3).

Because of the large number of variables associated with two-phase systems (e.g. liquid and vapor pressures, densities, temperatures and mixture quality), they are notoriously difficult to characterize. Fortunately, their complicated nature has made them a popular research topic and a wealth of empirical correlations have been developed to aid in predicting and explaining the behavior of two-phase flows. There are also a number of simplified analytical models available. In the following section, terms and theories 
necessary to understanding basic two-phase flows will be discussed, followed by a brief overview of the most common relevant two-phase modeling techniques.

\section{II.2.1 Two-Phase Governing Equations}

A brief description of the equations governing two-phase flows will be useful. These equations apply to the internal flow of liquid and vapor despite the relative distribution of the phases. A generic representative schematic of such a system is shown in Figure 3 below.

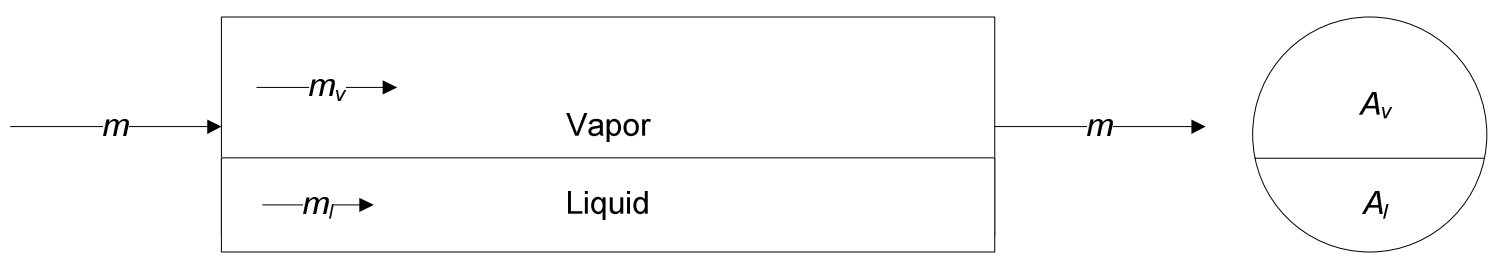

Figure 3. Generic two-phase flow schematic

First, the total mass flow rate is the sum of the liquid and vapor mass flow rates:

$$
\dot{m}=\dot{m}_{v}+\dot{m}_{l}
$$

The quality is defined as the ratio of the vapor mass flow rate to the total mass flow rate:

$$
x=\frac{\dot{m}_{v}}{\dot{m}}
$$


The total mass flux is identical to that of a single phase flow and is given by the ratio of the total mass flow rate to the total cross sectional area of the duct:

$$
G=\frac{\dot{m}}{A}
$$

Similar to the mass flow rate, the total cross sectional area of the annulus is equal to the sum of the areas occupied by the vapor and liquid phases:

$$
A=A_{v}+A_{l}
$$

The void fraction is defined as the fraction of the cross sectional area of the annulus occupied by vapor and is given by

$$
\alpha=\frac{A_{v}}{A}
$$

The velocity of the vapor phase at any location is given by

$$
u_{v}=\frac{G x}{\rho_{v} \alpha}
$$

Likewise, the velocity of the liquid at any location is given by 


$$
u_{l}=\frac{G(1-x)}{\rho_{l} \alpha}
$$

The slip ratio $S$ is defined as the ratio of liquid to vapor velocities:

$$
S=\frac{u_{l}}{u_{v}}
$$

These definitions will be useful in the two-phase models that follow.

\section{II.2.2 Flow Regimes}

Before modeling the hydrodynamic and thermal characteristics of any two-phase flow, it is necessary to have some knowledge of the orientation of the phases within the annulus. Flows are typically classified into "regimes" based on the relative distribution of phases. Examples of typical flow regimes are: bubbly flow, slug flow and annular flow. It is imperative that some assumptions or conclusions about which regime a flow is classified under be made prior to attempting to characterize specific flow properties. The regime will depend on a number of parameters that include but are not limited to: mass flow rate, geometry and orientation of the annulus, heat flux, pressure, and fluid. Significant

differences are observed in flow regimes in vertical and horizontal annuli as shown in Figure 4 and Figure 5 below: 


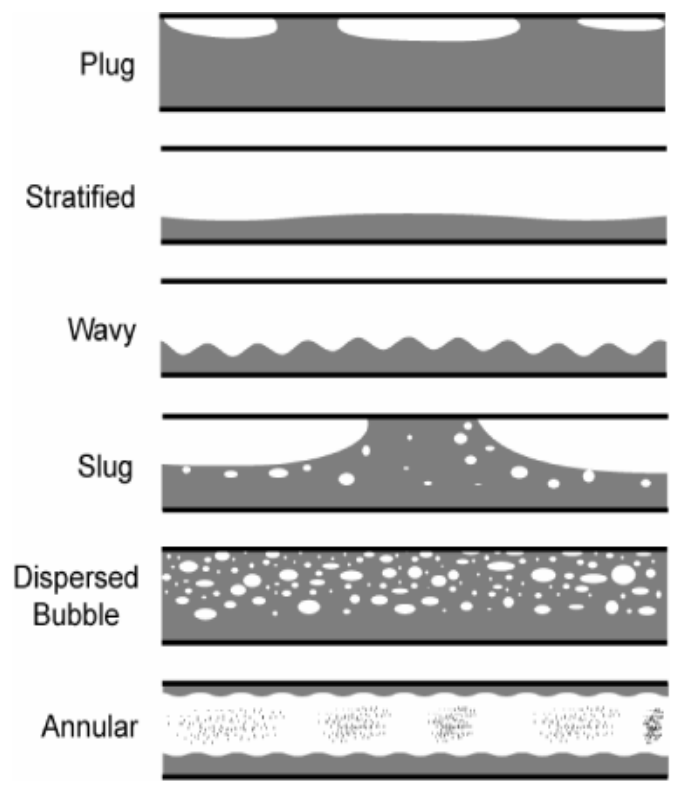

Figure 4. Typical flow regimes encountered in horizontal flow (4)

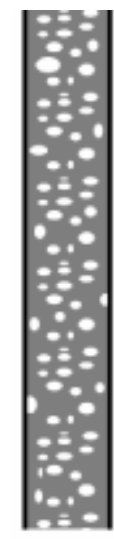

Bubbly

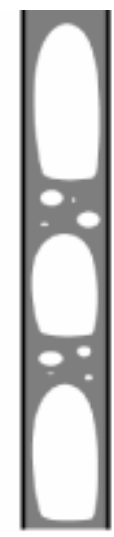

Slug

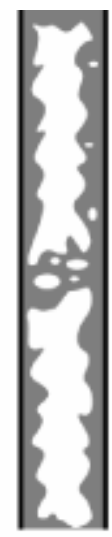

Churn

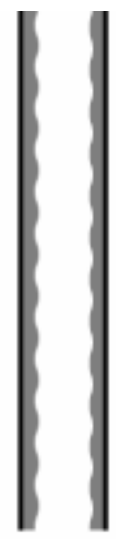

Annular

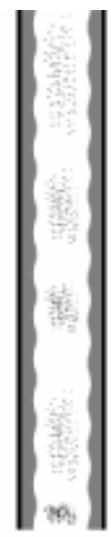

Wispy-Annular

Figure 5. Typical vertical two-phase flow regimes (4)

Notice that because of the presence of gravity out of plane with the axis of the annulus in horizontal flows, the gas phase tends to collect near the top of the annulus. However, as the mass flux increases, the buoyant forces become increasingly small compared to inertial forces reducing the tendency of the flow to be stratified. It is imperative that there 
be some knowledge or assumption regarding which of the above flow regimes is present prior to predicting the characteristics of any two-phase flow.

\section{II.2.3 Critical Heat Flux and Dryout}

The critical heat flux ("CHF") is defined as the heat load above which the liquid phase will no longer wet the walls of the annulus. If the applied heat flux exceeds this value, the flow is classified as film boiling rather than flow boiling. The point at which the critical heat flux is reached in the annulus is termed the "dryout" point. The most notable consequence of dryout is a significant increase in wall temperature and decrease in heat transfer coefficient.

Boiling of a stagnant fluid is referred to as "pool boiling" whereas that of a moving fluid is termed "flow boiling." A basic understanding of the critical heat flux in pool boiling will be useful prior to explaining that for flow boiling. Shown in Figure 6 below is a typical pool boiling curve (not specific to nitrous oxide). The points labeled A, B and C

represent points at which large physical changes in the heat transfer mechanism occur in the system. 


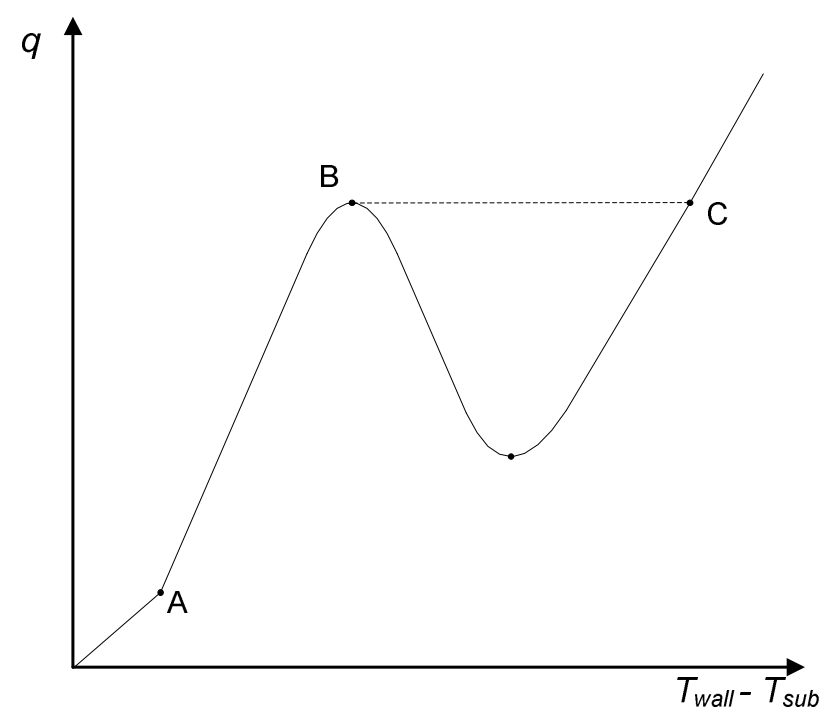

Figure 6. Generic pool boiling curve

Below point $\mathrm{A}$, natural convection is the dominant form of heat transfer. In this region, the applied heat flux is not sufficient to induce nucleation of vapor bubbles on the heated surface. Between points $\mathrm{A}$ and $\mathrm{B}$, the wall temperature is significantly above the saturation temperature and vapor bubbles form on the surface. In this region, heat transfer is due to the latent heat of vaporization required to form these vapor bubbles. This type of heat transfer is termed nucleate boiling. The solid line between points $\mathrm{B}$ and $\mathrm{C}$ is the transition boiling regime. It is particularly difficult to maintain a system in this region unless the temperature of the heated surface is controlled. Typically, a system will bypass the transition boiling regime, taking the dashed line between points B and C instead. Above point $\mathrm{C}$, a vapor blanket covers the heated surface, preventing liquid from wetting it. The low thermal conductivity of the vapor causes a sharp decrease in the heat transfer coefficient and a large increase in surface temperature. Points $\mathrm{B}$ and $\mathrm{C}$ correspond to the 
critical heat flux. It is generally desirable that systems operate below this point to avoid overheating.

A figure similar to the pool boiling curve that is more commonly used with two-phase flow rather than pool boiling is given in Figure 7 below. For the sake of simplicity, it will be termed the "flow boiling curve."

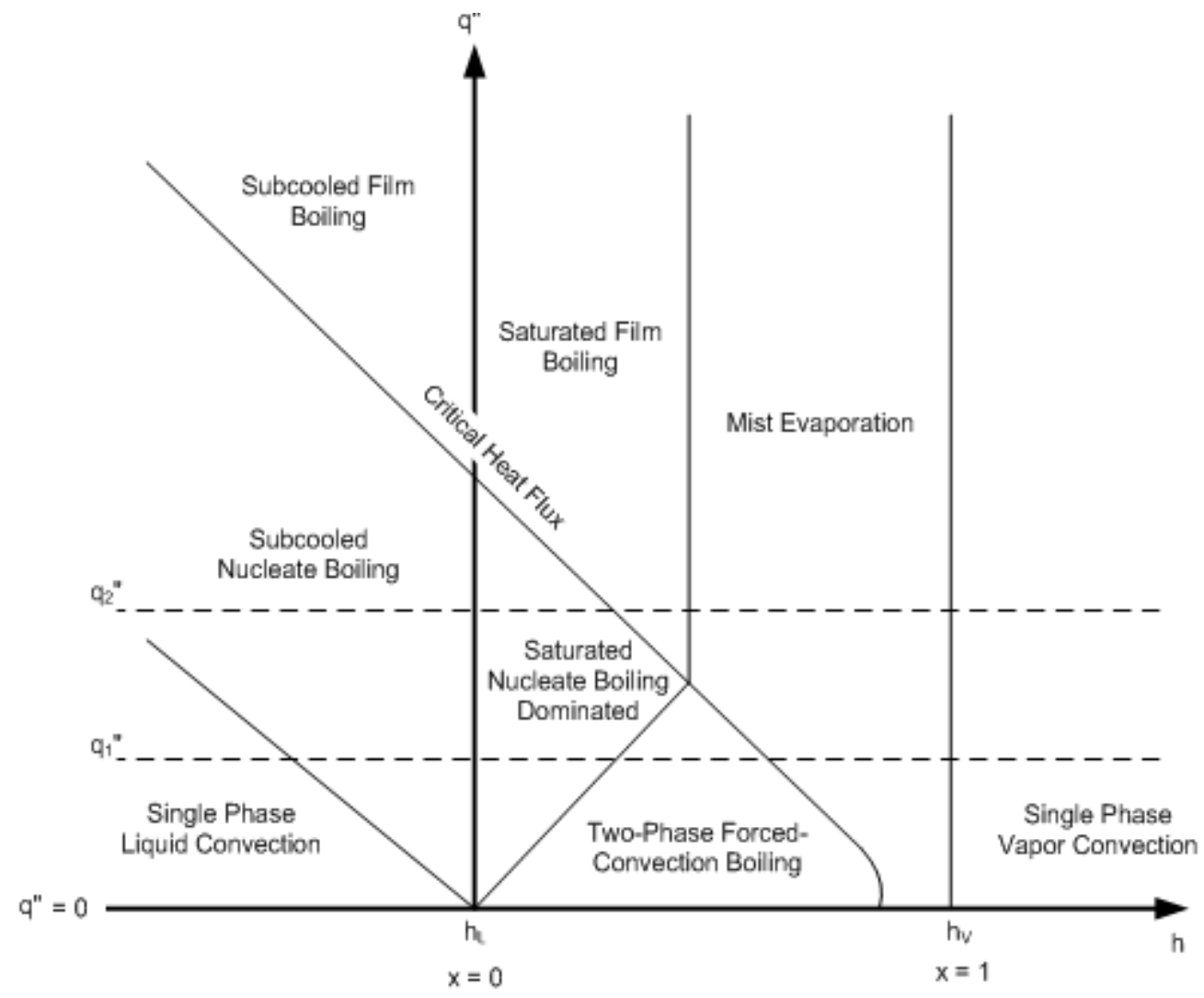

Figure 7. Flow boiling curve at constant mass flux and pressure (5)

In flow boiling, the critical heat flux is a function of thermodynamic quality. As a fluid moves through a heated annulus and is evaporated, the quality increases. This results in a decrease in CHF. If the fluid remains in the annulus long enough, the applied heat flux will eventually exceed the critical heat flux. The location in the annulus at which this 
occurs is called the "dryout" point. As was the case in pool boiling, dryout in flow boiling results in an increase in surface temperature and is generally avoided.

There are two types of dryout. If the fluid is low quality at the point of dryout, inverted annular flow (IAF) occurs in which a liquid core is surrounded by a vapor ring. Typically, the fluid is in the bubbly or slug flow regimes prior to IAF dryout. This scenario corresponds to $q_{2}$ ” in Figure 7 above. The second type of dryout is termed mist flow in which the fluid simply breaks into small droplets and is suspended in a vapor bulk. This type of dryout typically occurs at high qualities when the fluid is in the annular flow regime prior to mist flow dryout. This corresponds to $q_{1}$ " in Figure 7. Note that heat transfer coefficients in the mist flow regime are considerably higher than those in the IAF regime. Similarly, the observed wall temperatures are much lower in mist flow than IAF. This is due to the fact that in mist flow liquid is able to intermittently wet the walls of the annulus which is not the case in IAF.

Before modeling the heat transfer or hydrodynamic properties of any system, it is imperative to know or assume where on the film boiling diagram the system lays. Specifically, some assumptions or conclusions about whether or not the applied heat flux exceeds the CHF must be made. If it is presumed or concluded that the applied heat flux exceeds the CHF, then dryout has already occurred and it is necessary to discern which type of dryout it is (i.e. IAF or mist flow). If the applied heat flux is less than the CHF, then it necessary to determine which flow regime is present. Only after proper 
classification has been accomplished can the heat transfer coefficients and pressure drop in the annulus be characterized. This is summarized in Figure 8 below.

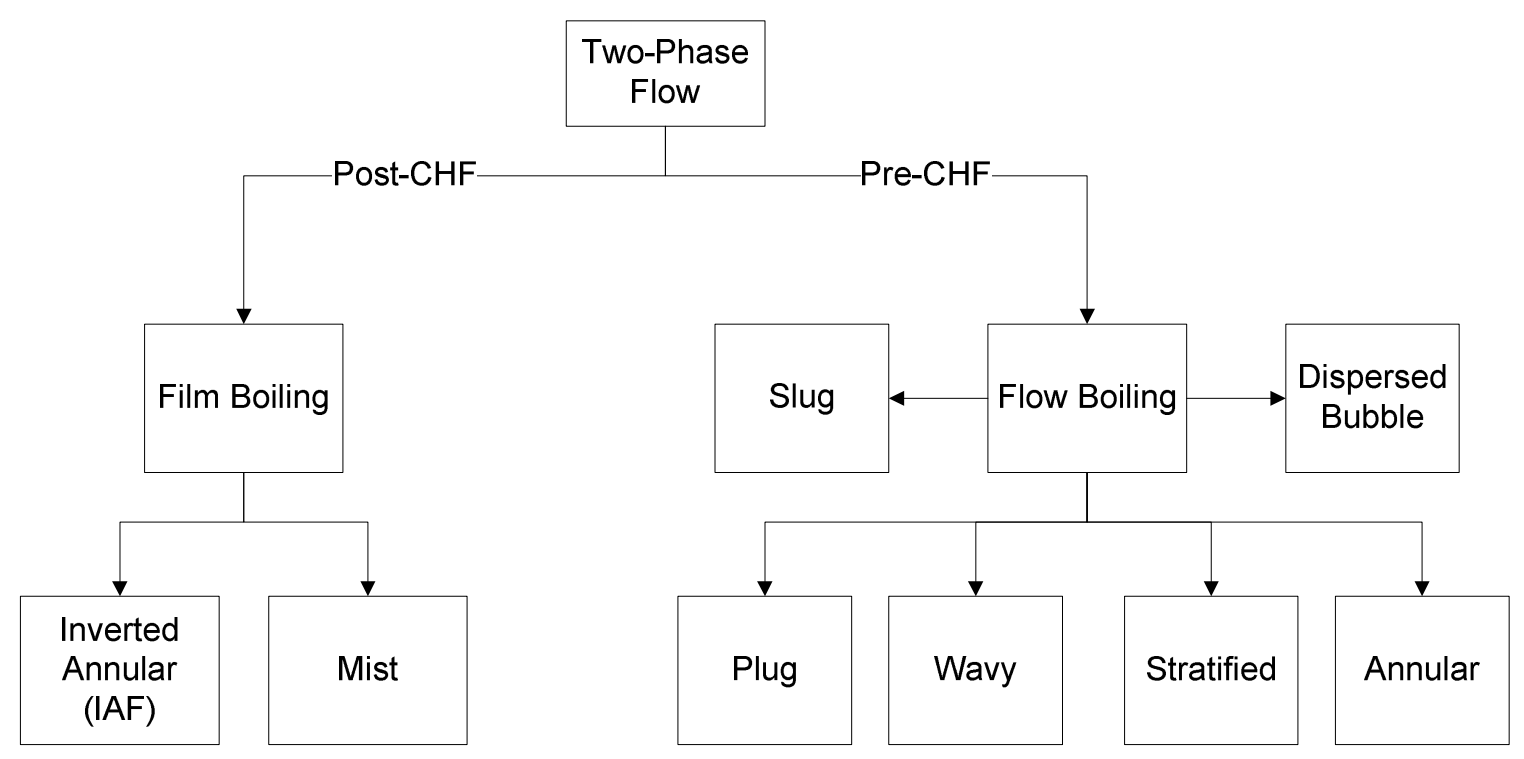

Figure 8. Two-phase flow characterization flowchart

Unfortunately, most of the reputable methods of determining CHF are empirically based correlations that are applicable to steam-water mixtures only.

\section{II.2.4 Thermal Equilibrium}

In many two-phase flow models and correlations, the fluid is assumed to be in "thermal equilibrium" meaning that both the liquid and vapor phases exist at the saturation temperature. This is not typical as in nearly all physical two-phase systems the liquid phase will be slightly subcooled while the vapor phase is slightly superheated. This state is termed "thermal non-equilibrium." It has been shown that flows with relatively high mass fluxes $\left(G>2500 \mathrm{~kg} / \mathrm{m}^{2} \mathrm{sec}\right)$ and low dryout qualities tend to more closely resemble 
the case of thermal equilibrium (6). It is important to note that some error will be introduced by implementing a model that assumes thermal equilibrium. However, this assumption also offers a significant simplification to the modeling process as it provides a convenient link between temperature and pressure.

\section{II.2.5 Pre-Existing Two-phase Heat Transfer Models}

Two methods are commonly used for characterizing two-phase flow. First, many empirical correlations have been developed but tend to be applicable only to the specific conditions (i.e. fluid, annulus geometry, et cetera) for which they were developed. As this method is highly dependent on the ability to gather experimental data, the conditions for which these correlations exist are relatively limited (i.e. steam-water mixtures in vertical round tubes). Nevertheless, they have proven to be relatively accurate when used appropriately. The second method of characterizing two-phase systems is analytical modeling. Unfortunately, this technique requires a large number of simplifying assumptions as these types of flows are inherently complex. In addition, the systems of equations that result from analytical modeling typically must be solved numerically. In general, empirical correlations are regarded as more convenient and, in most cases, more accurate and are therefore more widely used than analytical models. In any case, a brief discussion of the empirical and analytical models that are especially relevant to this study will be beneficial.

M.M. Shah proposed a semi-empirical correlation for predicting heat transfer coefficients of pre-CHF two-phase diabatic flows (7). This correlation is convenient as it is applicable 
to any fluid and to both vertical and horizontal flows. Its major limitation is that it only models the flow boiling of saturated mixtures prior to dryout. The correlation is based on four different heat transfer coefficients, each representing a different dominant heat transfer mechanism. The first represents the nucleate boiling regime in which vapor bubbles continuously form on the surface of the annulus and boil into the core of the flow. The second represents the convective boiling regime in which common singlephase convection is the dominant mode of heat transfer. The last two heat transfer coefficients represent the "bubble suppression" regime which can be roughly thought of as the regime in which both convective heat transfer and nucleate boiling are significant. Each of these heat transfer coefficients is a function of the single phase liquid heat transfer coefficient as determined by the Dittus-Boelter Nusselt number correlation and a group of non-dimensional parameters: (1) the Boiling number $B o,(2)$ the Froude number Fr and (3) the Convection number Co. The Shah correlation is highly regarded for its range of applicability and ease of use.

Chen gave another widely used and referenced heat transfer coefficient correlation for nucleate flow boiling (8). The basis for this correlation is the proposition that the heat transfer coefficient of any two-phase diabatic flow is an additive combination of the macroscopic and microscopic heat transfer mechanisms. From this hypothesis, Chen let the total heat transfer coefficient be equal to the sum of the convective heat transfer coefficient (macroscopic contribution) and the boiling heat transfer coefficient (microscopic contribution). 
Varone and Rohsenow developed an analytically based model to predict post-dryout mist flow heat transfer and hydrodynamic behavior of two-phase flows (6). Modeling mist flow as a suspension of liquid droplets in a gaseous bulk fluid, the total heat transfer coefficient was assumed to be a function of the heat transfer coefficients between the wall, the vapor and the entrained liquid droplets. While the model is analytically based, numerous empirical correlations are necessary to approximate values such as the turbulent friction factor and the single phase convective heat transfer coefficient. A primary advantage of this method is that it accounts for thermal non-equilibrium. However, it does result in a sizeable system of ordinary differential equations (ODEs) and algebraic expressions. Because this system of equations is so complex, it must be solved both numerically and iteratively.

One of the most widely referenced methods for determining the critical heat flux is the Groeneveld look-up table (9). In this model, CHF is tabulated as a function of pressure, mass flux, and quality. This table is simply a conglomeration a several banks of experimental data (approximately 40,000 data points). Unfortunately, the Groeneveld look-up table is only applicable to steam-water mixtures flowing in vertical round tubes.

Thome and Revellin developed a two-phase separated flow with interface exchange model for determining the critical heat flux in horizontal mini and microchannels (10). Assuming an annular flow regime exists, the CHF is defined as the point in which the height of the interfacial waves exceeds the liquid film thickness. This model makes no assumptions about the fluid and was developed only for flows in horizontal mini and 
microchannels (less than 3 millimeters in diameter). It assumes a saturated liquid boundary condition at the inlet of the annulus. It has been validated for R-113, R-245fa, R-134a and water. Unfortunately, the system of five non-linear ODEs that results from the governing equations is impossible to solve in closed form and cumbersome to solve numerically. This method has been referenced as the most accurate method for determining the $\mathrm{CHF}$ for non-aqueous flows in channels less than 3 millimeters in diameter (11). It is also advantageous as the hydrodynamic behavior of the liquid and gas phases are determined in the process of determining the critical heat flux.

\section{II.3 PREVIOUS HYBRID ROCKET TESTING AT CAL POLY}

In order to fully understand the Rayleigh experiments and their results, it is important that background information regarding the Cal Poly hybrid rocket be discussed. A general schematic and photograph of the system are shown in Figure 11 below. The primary elements of the rocket motor are the: (1) combustion chamber in which the oxidizer and fuel are combined and ignited, (2) De Laval nozzle through which the combustion products are accelerated to supersonic velocities, (3) coolant annulus through which twophase nitrous oxide flows, and (4) instrumentation system for monitoring temperature and pressure at critical locations. Note that two different nozzle geometries exist. The nozzle used in previous tests, termed the "two flow-paths" nozzle, includes two half circles coolant flow paths while that used in the experiments most relevant to this thesis, termed the "Rayleigh" nozzle, includes a nearly 360-degree coolant path. The Rayleigh nozzle is ideal for the experiments at hand as it more closely represents one-dimensional flow. The 
two flow-paths nozzle is shown in Figure 9 and the difference between the two is illustrated in Figure 10.

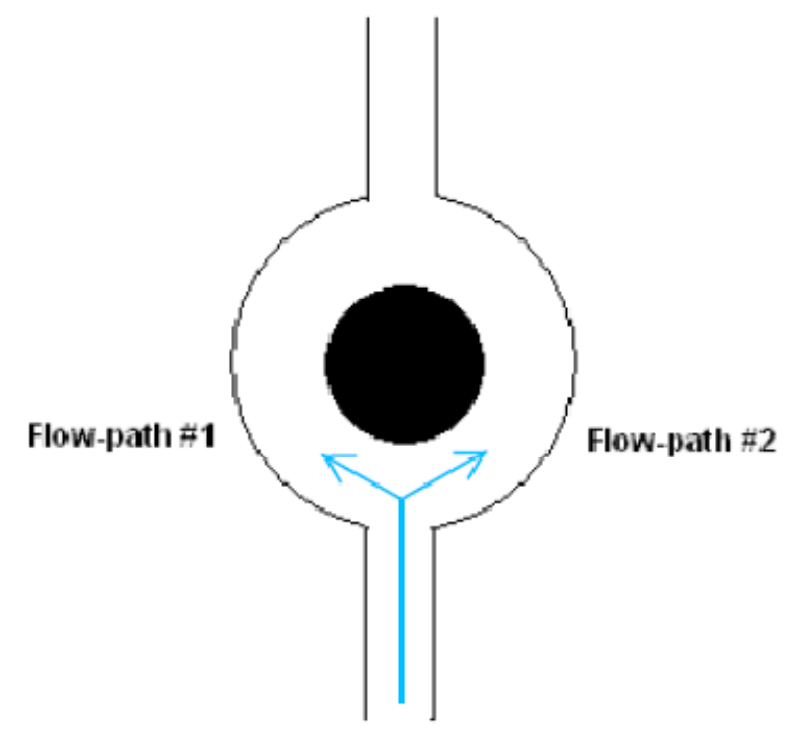

Figure 9. Top down schematic of two flow-paths nozzle geometry

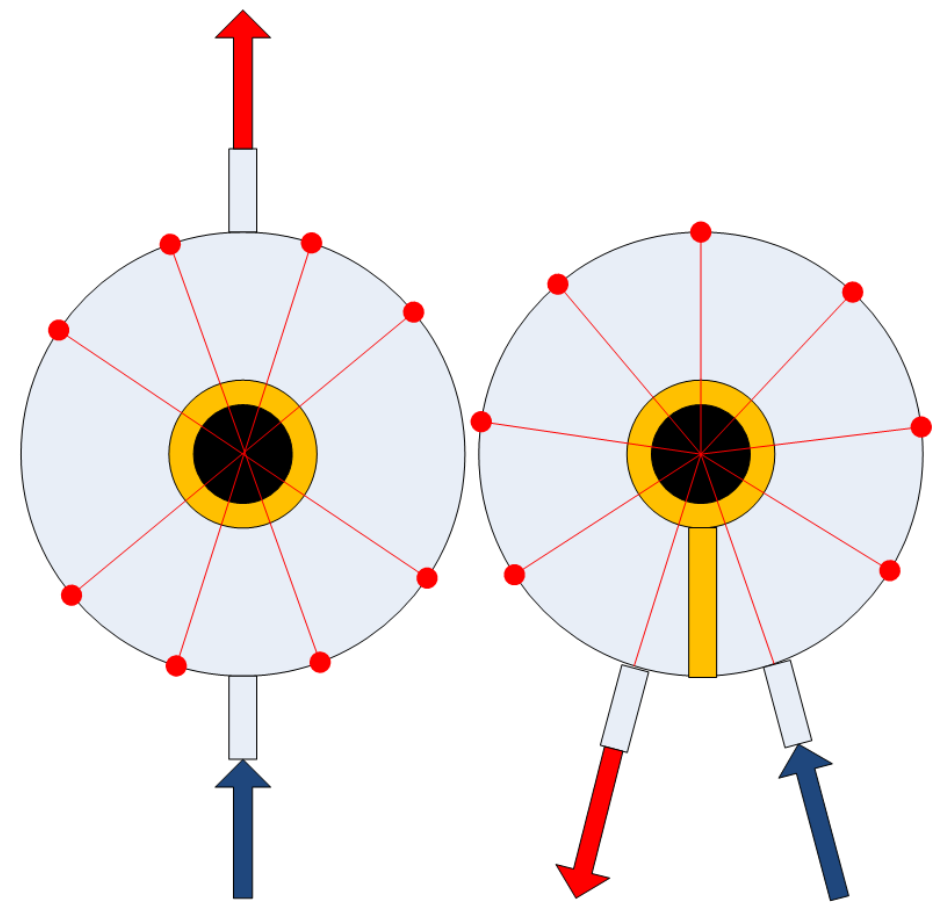

Figure 10. Top down view of the original nozzle (left) and the Rayleigh nozzle (right). Red lines represent measurement stations. 

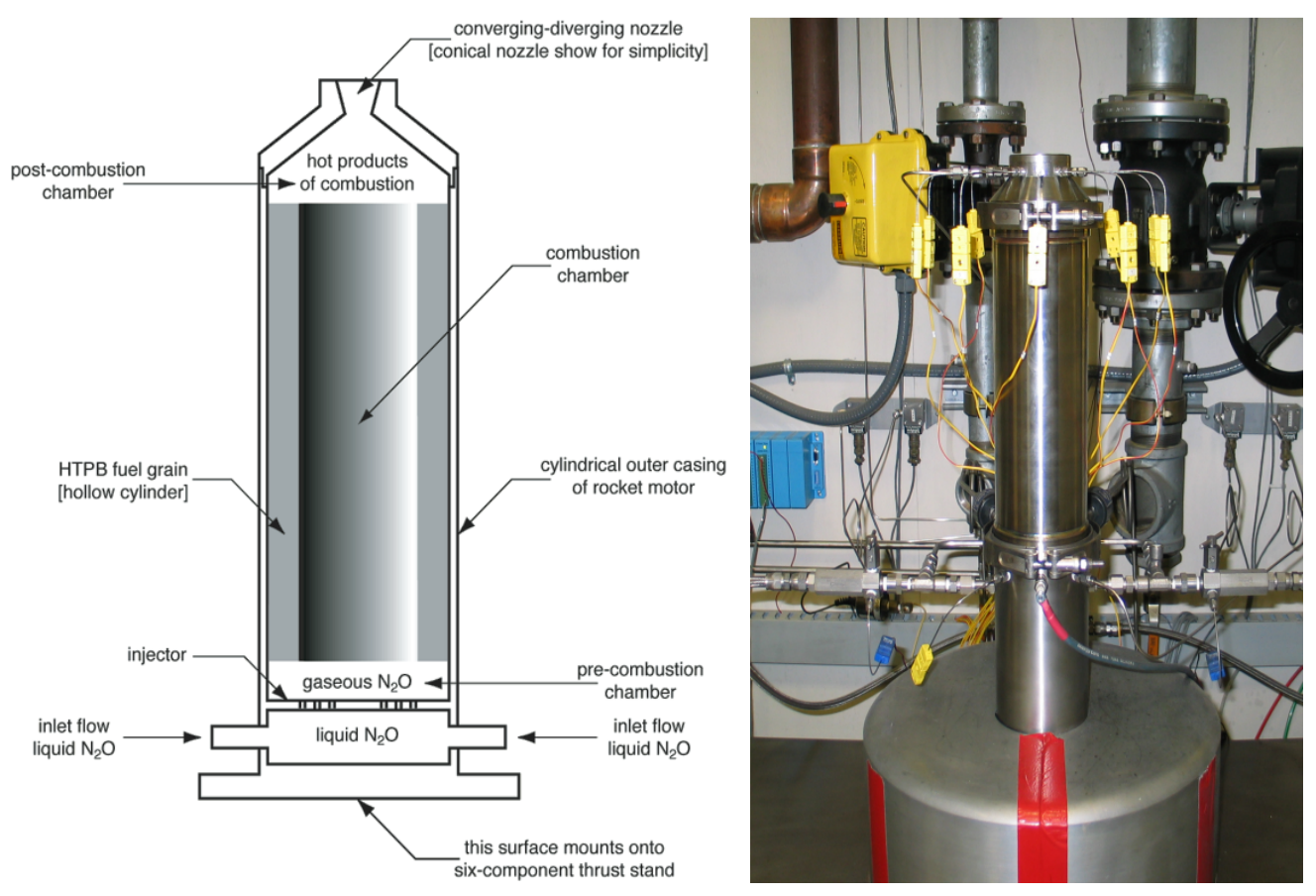

Figure 11. Cal Poly hybrid rocket (12)

A representative close-up of the two flow-paths nozzle and cooling system is shown in Figure 12.

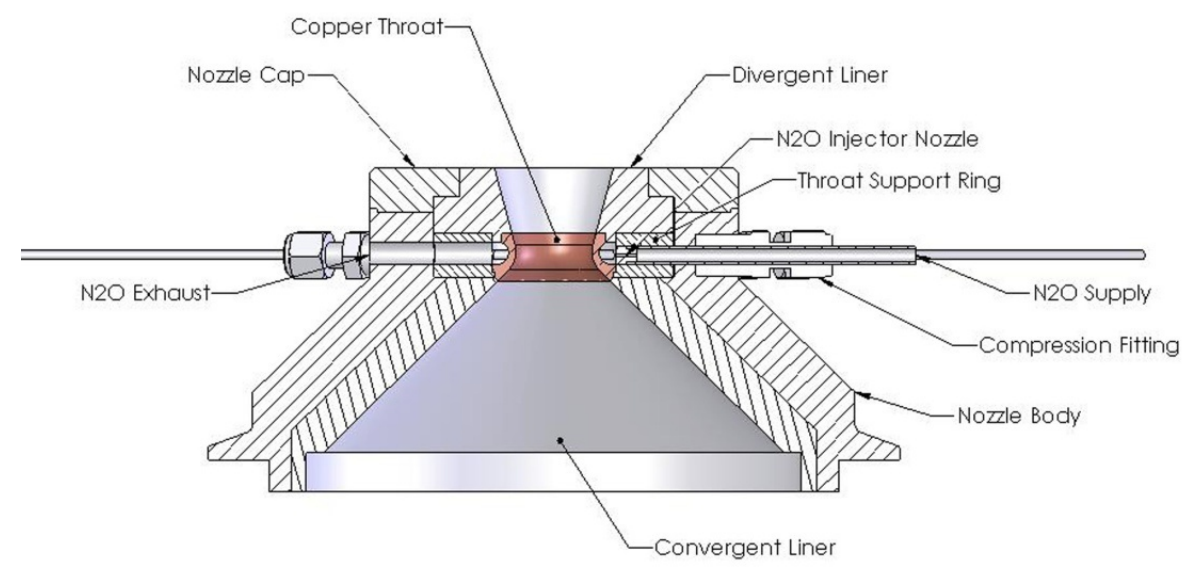

Figure 12. Cal Poly cooled converging-diverging nozzle (12) 
A top-down photograph of the two flow-paths nozzle and instrumentation system is shown in Figure 13 below. Coolant enters the flow path at the fitting on the right and exhausts through the fitting on the left.

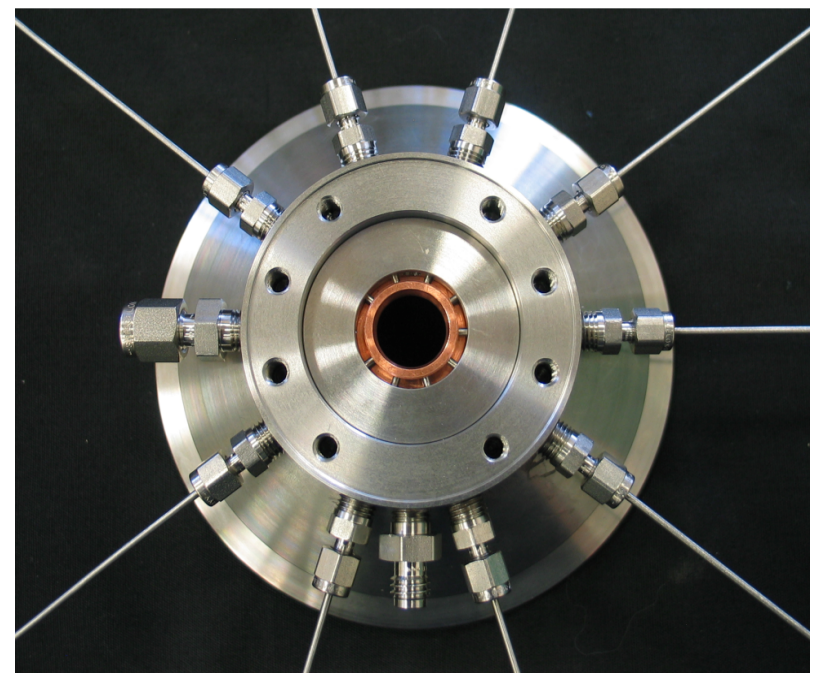

Figure 13. Top-down image of the two flow-paths nozzle and instrumentation (12).

The combustion chamber and De Laval nozzle are classified as the "hot side" of the system and the coolant annulus is considered the "cold side." Because the purpose of this thesis is to characterize the cold side of the hybrid rocket nozzle, only elements of the hot side that are pertinent to cold side analysis will be discussed. Most importantly, this section will explain methods of characterizing the amount of heat transfer from the hot side to the cold side. Of primary importance in this analysis is the copper ring separating the combustion gases from the coolant which can be seen in the center of the apparatus in Figure 13 (shown in its entirety in Figure 14 below). The combustion gases flow through the center section of the ring while the coolant flows through the annulus embedded circumferentially around the ring. The copper tab separates the coolant inlet and outlet. In 
this way, the copper ring offers the link between the hot side and the cold side of the system.

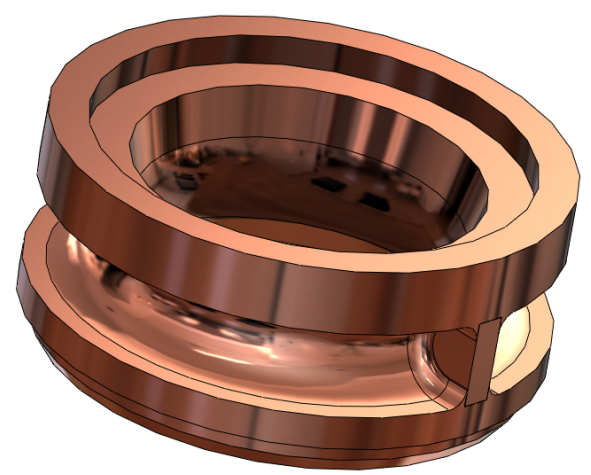

Figure 14. Rayleigh nozzle copper throat insert.

For each rocket fire, a comprehensive set of data is recorded which includes but is not limited to several copper temperatures, coolant temperatures and coolant pressures as well as chamber pressure and coolant mass flow rate. Note that this data is recorded timedependently over the course of the run and is therefore not necessarily steady state. A sample data output take from Run 50 is shown in graphical form in Figure 15 for an experiment using the two flow-paths nozzle. 


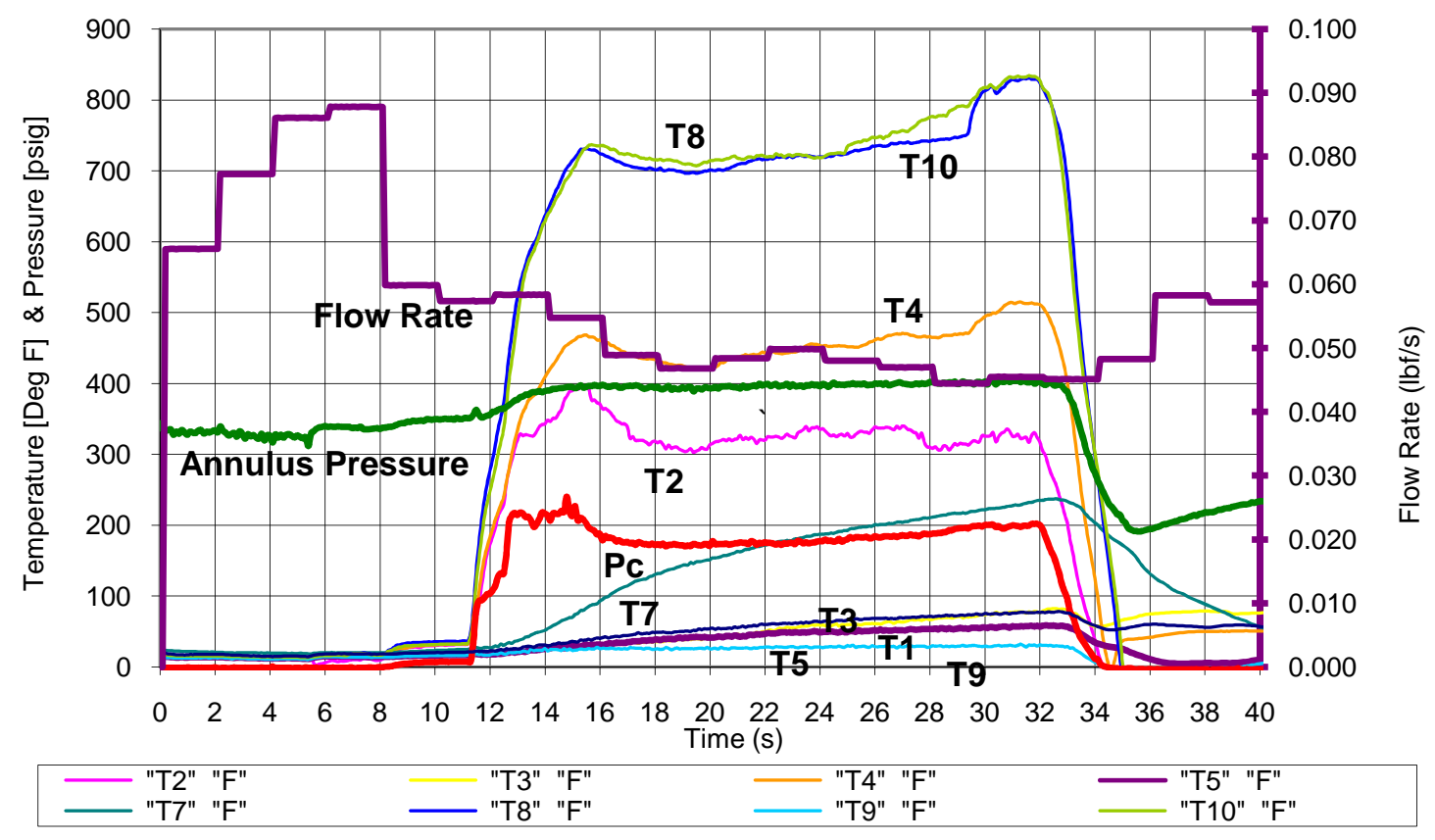

Figure 15. Sample data output from Cal Poly hybrid rocket tests

Note that the thermocouples used in this run measure temperatures of both gas and copper temperatures. The traces labeled $\mathrm{T} 1, \mathrm{~T} 3, \mathrm{~T} 5, \mathrm{~T} 7$ and $\mathrm{T} 9$ are measuring gas temperatures and $\mathrm{T} 2, \mathrm{~T} 4, \mathrm{~T} 8$ and $\mathrm{T} 10$ are measuring copper temperatures. The locations of these thermocouples within the nozzle are shown in Figure 16. 


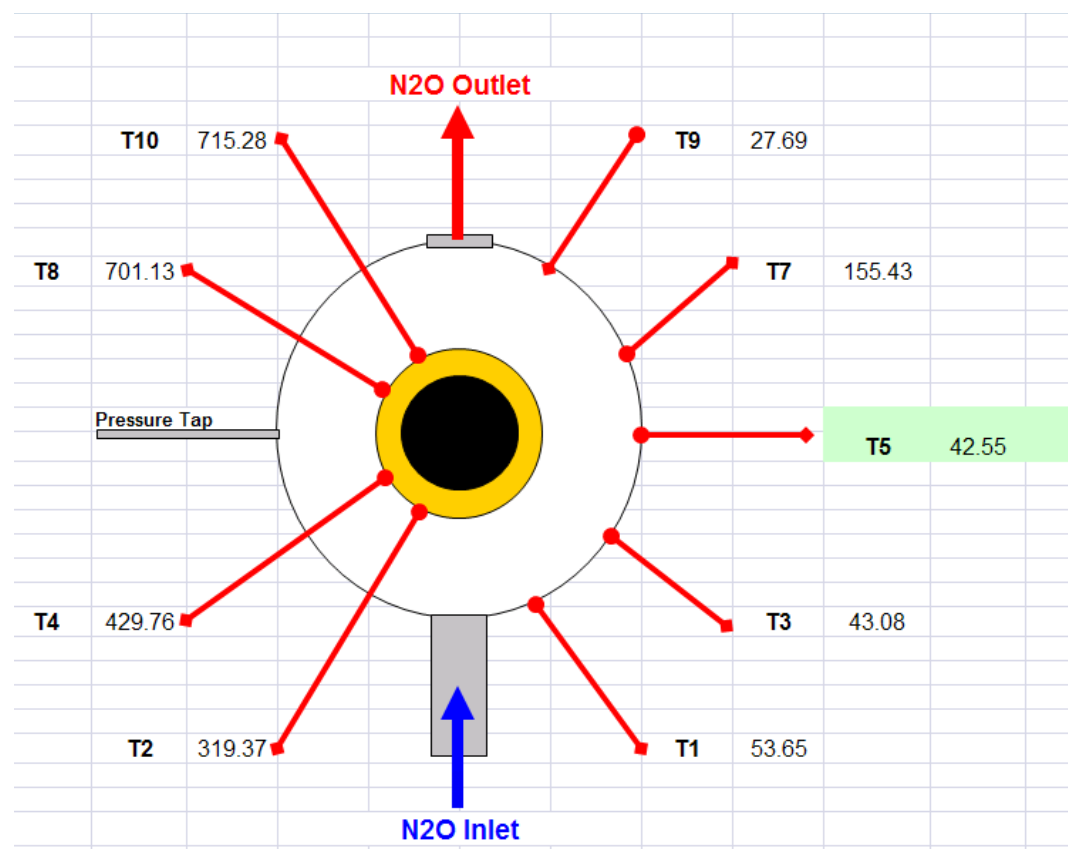

Figure 16. Top down schematic of the two flow-paths nozzle.

For each run, a region is identified in which all or most variables are relatively stable. This region is denoted as the steady state region and values are either averaged or a representative value is chosen in that region, yielding a single steady state value for each variable. In Figure 15, the region between approximately 14 and 30 seconds would be considered steady state. The analyses in the following sections do not attempt to model transient phenomena.

Several experiments were run previously with Plexiglas ${ }^{\circledR}$ as fuel, nitrous oxide as coolant and the two flow-paths nozzle. The measured steady state copper and coolant temperatures as a function of distance through the annulus for these relevant rocket fires are shown in Figure 17 and Figure 18. It is crucial to note that these experiments were run 
with a nozzle with dramatically different geometry than the Rayleigh nozzle as was discussed previously. This nozzle split the flow in two.

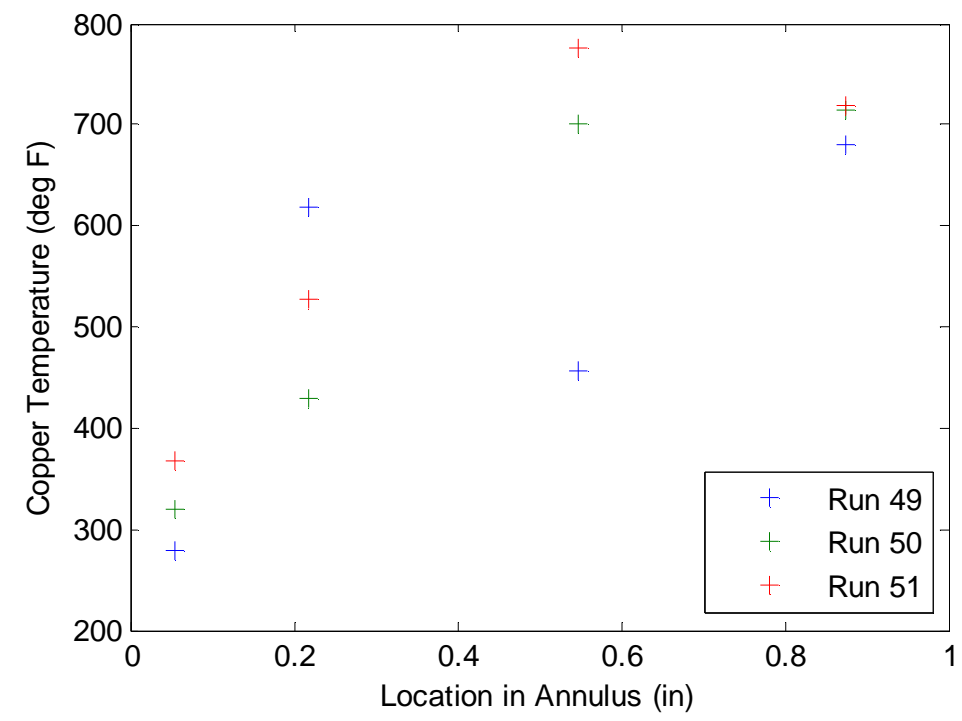

Figure 17. Copper temperatures through annulus in previous two-phase cooled tests

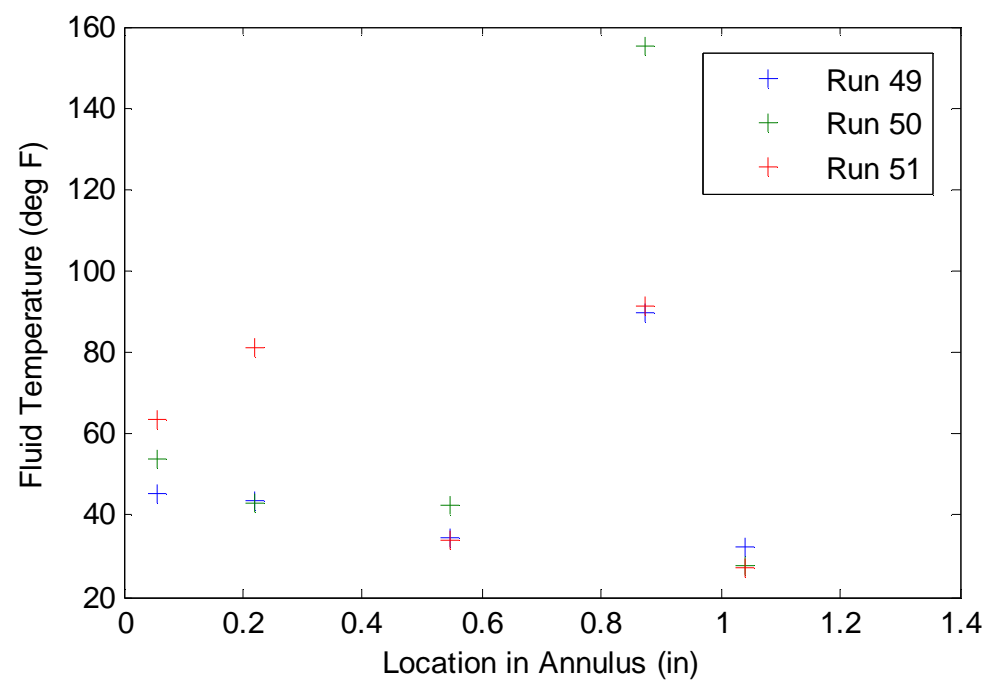

Figure 18. Fluid temperatures through annulus in previous two-phase cooled tests

The data presented above is utilized in the following analyses. 


\section{II.3.1 Hot Side Heat Transfer Characteristics}

In order to model the cold side of the system, it is first necessary to characterize the hot side heat transfer. Because of its high thermal conductivity relative to its surroundings, all of the heat from the combustion gases in the throat of the nozzle is assumed to be transferred into the copper ring. The coolant is then assumed to absorb all of this heat. In reality, some of the heat from the combustion gases is transferred to parts of the nozzle other than the copper ring. Consequently, this assumption results in an overprediction of the amount of heat transferred from the combustion gases into the coolant. Note that this is a very important assumption that affects all subsequent analyses and leads to some error in those analyses. This assumption is necessary as it makes these subsequent analyses possible.

In order to determine how much heat is transferred into the coolant, the heat transfer characteristics of the combustion gases must first be characterized. Two forms of hot side heat transfer are considered: (1) convection and (2) radiation. The total heat transfer is simply the sum of the individual heat transfer terms:

$$
q=q_{\text {convection }}+q_{\text {radiation }}
$$

Bartz formulated a simple method of approximating the convective heat transfer coefficient of combustion products (13). Assuming that (1) the Nusselt number can be written in the familiar Colburn-type form of the product of the Reynolds and Prandtl numbers, (2) the Prandtl number, specific heat and molecular viscosity do not vary 
significantly with temperature through the nozzle, and (3) an average mass flow rate across the cross sectional throat area can be used, it can be shown that the heat transfer coefficient at the throat of a con-di nozzle is given by

$$
h_{\text {hot }}=\frac{0.026}{D_{*}^{0.2}}\left(\frac{\mu^{0.2} C_{p}}{\operatorname{Pr}^{0.6}}\right)_{0}\left(\frac{P_{c} g}{C_{*}}\right)\left(\frac{D_{*}}{r_{c}}\right)^{0.1}\left(\frac{A_{*}}{A}\right)^{0.9} \sigma
$$

where

$$
\sigma=\frac{1}{\left[\frac{1}{2} \frac{T_{\text {wall }}}{T_{0}}\left(1+\frac{\gamma-1}{2} M^{2}\right)+\frac{1}{2}\right]^{0.8-(w / 5)}\left[1+\frac{\gamma-1}{2} M^{2}\right]^{w / 5}}
$$

In equation (3.10), the subscript " 0 " denotes a property evaluated at stagnation (or combustion chamber) conditions, “*” denotes a property evaluated at sonic (or throat) conditions, and "wall" denotes conditions at the copper wall. $D$ is the nozzle diameter, $P_{c}$ is the chamber pressure, $A$ is the cross-sectional area of the nozzle, $r_{c}$ is the radius of curvature, $\mu$ is the dynamic viscosity, $\gamma$ is the ratio of specific heats, $T$ is the temperature, $g$ is the gravitational constant, $c$ is the speed of sound, $P r$ is the Prandtl number, and $M$ is the Mach number.

Given the chamber pressure and oxidizer to fuel ratio $(\mathrm{O} / \mathrm{F})$ for any combustion process, the properties required to evaluate equations (3.10) and (3.11) above can be determined using a computer software package known as Thermodynamic Equilibrium Program ("TEP"). In this way, because both chamber pressure and O/F are monitored in every test 
at Cal Poly, the convective heat transfer coefficient of the combustion gases at the throat of the nozzle can also be evaluated. For a Plexiglas ${ }^{\circledR}$ and $\mathrm{N}_{2} \mathrm{O}$ test at the Cal Poly facility, a typical value of $h_{h o t}$ at the throat is $2.5 \mathrm{E}-3 \mathrm{BTU} / \mathrm{in}^{2} \mathrm{sec}^{\circ} \mathrm{R}$. Note that TEP is also capable of predicting the combustion gas temperature at the throat of the nozzle, $T^{*}\left(T_{h o t}\right)$, for a given chamber pressure, $\mathrm{O} / \mathrm{F}$ and reactants.

Because of the varying diameter and cross sectional area of the nozzle, the magnitude of the heat transfer coefficient will peak at the throat of the nozzle and drop off quickly leading up to and leaving the throat. Figure 19 shows this general trend. However, to maintain simplicity in this analysis, the heat transfer coefficient is assumed to remain constant at the throat value. This results in a constant heat flux into the copper and a total heat load that is larger than the actual heat load.

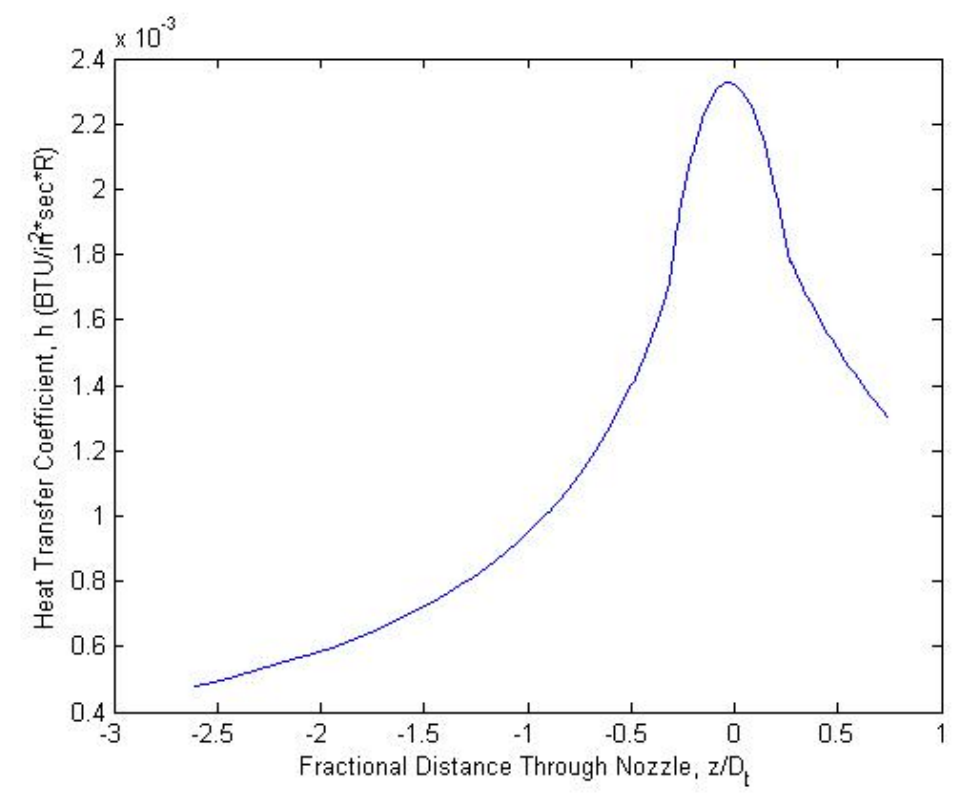

Figure 19. Bartz heat transfer coefficient distribution through the converging-diverging nozzle. 
The total convective heat transfer is then given by

$$
q_{\text {convection }}=h_{\text {hot }} A_{\text {hot }}\left(T_{\text {hot }}-T_{C u}\right)
$$

where $A_{\text {hot }}$ is the total area of the copper ring exposed to the combustion gases, $T_{h o t}$ is the combustion gas temperature at the throat and $T_{C u}$ is the copper temperature. As mentioned earlier, for a given chamber pressure and O/F, TEP can predict $T_{\text {hot }} . A_{\text {hot }}$ is a known quantity from the geometry of the nozzle. Without considering heat transfer into and out of the copper ring simultaneously, it is impossible to determine the wall temperature. In this way, for a precise estimate of the convective heat transfer, the hot and cold sides of the copper ring must be analyzed in conjunction with each other. This issue will be addressed in subsequent sections but for approximate calculations a value of $500^{\circ} \mathrm{F}$ is used for the copper wall temperature. This temperature was set as a design point as above it the yield strength of copper decreases appreciably. Based on these assumptions, a typical value of convective heat flux into the coolant for this system is approximately $10^{7} \mathrm{~W} / \mathrm{m}^{2}$.

The equation governing radiation heat transfer is given by

$$
q_{\text {radiation }}=A_{\text {hot }} \sigma\left(\varepsilon_{g} T_{\text {hot }}{ }^{4}-\alpha_{g} T_{C u}{ }^{4}\right)
$$


where $\sigma$ is the Boltzmann constant, and $\varepsilon_{g}$ and $\alpha_{g}$ are the emissivity and absorptivity of the combustion gases respectively. As was the case for convection, the values of $A_{h o t}$ and $T_{\text {hot }}$ are known.

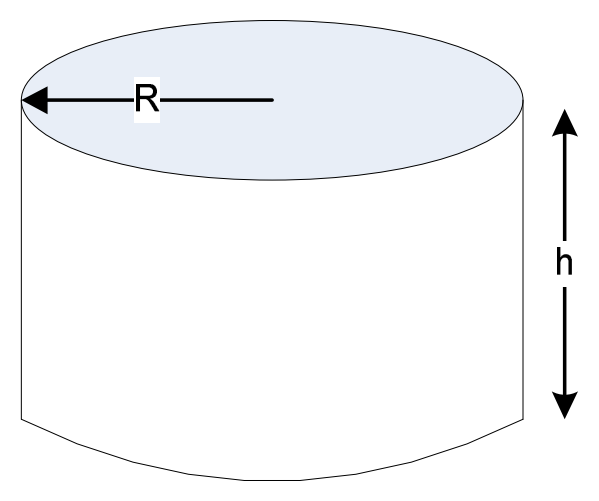

Figure 20. Control volume for radiation heat transfer in nozzle throat

For the stoichiometric combustion of Plexiglas ${ }^{\circledR}$ and $\mathrm{N}_{2} \mathrm{O}$, the significant combustion products are carbon dioxide $\left(\mathrm{CO}_{2}\right)$, water $\left(\mathrm{H}_{2} \mathrm{O}\right)$ and carbon monoxide $(\mathrm{CO})$. Note that both hydrogen and nitrogen are present in the combustion products but their radiative effects have been shown to be insignificant compared to the other products and are therefore neglected (14). The emissivity of the mixture of these three species can be approximated by the sum of their individual emissivities less a value to account for interspecies radiative interactions:

$$
\varepsilon_{g}=\varepsilon_{C O}+\varepsilon_{C O_{2}}+\varepsilon_{\mathrm{H}_{2} \mathrm{O}}-\Delta \varepsilon
$$

Modeling the throat of the nozzle as a cylinder as shown in Figure 20, the effective beam length is defined as (14) 


$$
L_{e}=\frac{4 V_{c y l}}{A_{c y l}}=2 R
$$

For an $\mathrm{O} / \mathrm{F}$ ratio of approximately 2.0 the mole fraction $y$ of each species is output by TEP as

$$
\begin{aligned}
& y_{\mathrm{CO}_{2}}=0.029 \\
& y_{\mathrm{H}_{2} \mathrm{O}}=0.085 \\
& y_{\mathrm{CO}}=0.339
\end{aligned}
$$

Note that this $\mathrm{O} / \mathrm{F}$ ratio is used because it is very rich mixture which results in a large amount of unburnt content, thus enhancing radiative effects. In this manner, it is a good "worst case scenario" to study radiative effects. The partial pressures are then given by

$$
\begin{gathered}
P_{\mathrm{CO}_{2}}=y_{\mathrm{CO}_{2}} P^{*} \\
P_{\mathrm{H}_{2} \mathrm{O}}=y_{\mathrm{H}_{2} \mathrm{O}} P^{*} \\
P_{\mathrm{CO}}=y_{\mathrm{CO}} P^{*}
\end{gathered}
$$

where $P^{*}$ is the static pressure of the mixture at the throat and is output by TEP based on $\mathrm{O} / \mathrm{F}$ and chamber pressure. Knowing the partial pressures, the effective beam length, and the static gas temperature at the throat $T^{*}$ (also given by TEP), the emissivities of the individual species are given by graphical or tabulated empirical data. In the case of water 
and carbon dioxide, this information is taken from published data tables (14), giving the following emissivities at atmospheric pressure

$$
\begin{aligned}
& \left.\varepsilon_{\mathrm{CO}_{2}}\right|_{1 \mathrm{~atm}}=0.017 \\
& \left.\varepsilon_{\mathrm{H}_{2} \mathrm{O}}\right|_{1 \mathrm{~atm}}=0.011
\end{aligned}
$$

In the throat of the nozzle the static pressure is typically around 14 atmospheres. Therefore, the values listed above must be modified using correction factors from published data tables (14). Based on the partial pressure of each species, the total pressure of the mixture and the effective beam length, the correction factors are

$$
\begin{aligned}
& C_{\mathrm{CO}_{2}} \approx 2 \\
& C_{\mathrm{H}_{2} \mathrm{O}} \approx 2
\end{aligned}
$$

Note that the scale on the figures from which these values were taken was not large enough to compensate for total pressures as high as 14 atmospheres. The above correction factors were therefore conservatively approximated. The corrected emissivities of water and carbon dioxide are then given by

$$
\begin{gathered}
\varepsilon_{\mathrm{CO}_{2}}=\left.C_{\mathrm{CO}_{2}} \varepsilon_{\mathrm{CO}_{2}}\right|_{1 \mathrm{~atm}}=0.034 \\
\varepsilon_{\mathrm{H}_{2} \mathrm{O}}=\left.C_{\mathrm{H}_{2} \mathrm{O}} \varepsilon_{\mathrm{H}_{2} \mathrm{O}}\right|_{1 \mathrm{~atm}}=0.022
\end{gathered}
$$


The factor to account for the interaction of $\mathrm{CO}_{2}$ and $\mathrm{H}_{2} \mathrm{O} \Delta \varepsilon$ is evaluated using similar tables which give a value of virtually zero for this term. Unfortunately, the tables and graphs used for evaluating the emissivities of water and carbon dioxide are not available for carbon monoxide. Instead, $\mathrm{CO}$ is assumed to be a transparent gas and the following expression is used (15)

$$
\begin{gathered}
\varepsilon_{C O}=\frac{(3.66 E 6) f_{01}}{\omega_{01}}\left(\frac{1.44 \omega_{01}}{T_{h o t}}\right)^{4} \frac{273}{T_{h o t}}\left(\frac{e^{\left(\frac{-1.44 \omega_{01}}{T_{g}}\right)}}{1-e^{\left(\frac{-1.44 \omega_{01}}{T_{g}}\right)}}\right)[1 \\
\left.+8 r\left(\frac{e^{\left(\frac{-1.44 \omega_{01}}{T_{g}}\right)}}{1-e^{\left(\frac{-1.44 \omega_{01}}{T_{g}}\right)}}\right)\right] P_{C O} L_{e}
\end{gathered}
$$

where

$$
r=\frac{f_{02}}{f_{01}}\left(1+e^{\left(\frac{-1.44 \omega_{01}}{T_{h o t}}\right)}\right)
$$

and $f_{01}, f_{02}$ and $w_{01}$ are constants specific to the fluid. For the case of $\mathrm{CO}$, they are equal to 1.1E-5, 7.5E-8 ad $2143 \mathrm{~cm}^{-1}$ respectively. Evaluating this expression gives a value of 0.08 for the emissivity of $\mathrm{CO}$. No expression for the interaction of $\mathrm{CO}$ with either $\mathrm{H}_{2} \mathrm{O}$ or $\mathrm{CO}_{2}$ is available. However, since the interaction of $\mathrm{H}_{2} \mathrm{O}$ and $\mathrm{CO}_{2}$ was found to be negligible, the same is assumed for all other interactions. The total emissivity of the mixture is then given by 


$$
\varepsilon_{g}=\varepsilon_{C O}+\varepsilon_{C_{2}}+\varepsilon_{H_{2} O}-\Delta \varepsilon=0.135
$$

Similar methods are used to evaluate the absorptivity of carbon dioxide and water. However, no convenient methods are available for evaluating the absorptivity of carbon monoxide so this term is neglected. The absorptivity of the mixture is then approximated as the sum of that of carbon dioxide and water and is found equal to approximately 0.48 .

The radiative heat transfer can then be evaluated using equation (3.13). For a chamber pressure of $200 \mathrm{psi}$ and an $\mathrm{O} / \mathrm{F}$ of 2.0 and assuming a wall temperature of $500^{\circ} \mathrm{F}$, the radiative heat flux is approximately $10^{5} \mathrm{~W} / \mathrm{m}^{2}$. This is two orders of magnitude smaller than that of convection which indicates that radiation can be neglected.

A considerable number of assumptions were necessary to reach the above result. In order to promote confidence in the assumption that radiation heat transfer is negligible, a worst case scenario is also considered. In this case, the gases would not absorb any of the radiation from the copper wall $\left(\alpha_{g}=0\right)$, and would emit as much energy as a blackbody $\left(\varepsilon_{g}=1\right)$. In this case, the radiative heat transfer is given by

$$
q_{\text {radiation,max }}=A_{\text {hot }} \sigma T_{\text {hot }}{ }^{4}
$$

Evaluating equation (3.31) for a gas temperature of $3000^{\circ} \mathrm{F}$ (typical of $2.0 \mathrm{O} / \mathrm{F}$ and 200 psia chamber pressure) gives a maximum possible radiative heat flux of approximately $8 \mathrm{E} 5 \mathrm{~W} / \mathrm{m}^{2}$ which is still two orders of magnitude smaller than the convective heat flux. 
The results of both radiation analyses give confidence in the assumption that radiation is negligible compared to convection in this system. In all subsequent analyses, the radiative heat transfer will not be considered and the total heat transfer will be equal to that of convection only.

\section{II.3.2 Biot Number Analysis}

After establishing the magnitude of the heat transfer from the hot side, modeling of the cold side or the coolant system can begin. The geometry of the coolant annulus itself is relatively complicated and certain simplifying assumptions are necessary to make modeling the system possible. One assumption that would make analysis especially convenient is that of a uniform copper temperature. What is most critical to making this assumption acceptable is that the conduction through the copper ring is negligible compared to the convective heat transfer to and from it. The relative temperature uniformity is assessed via the Biot number which is the dimensionless parameter representing the ratio of heat transfer by convection to heat transfer by conduction within the copper. The Biot number is defined as

$$
B i \equiv \frac{h L_{c}}{k}
$$

where $h$ is the convective heat transfer coefficient, $L_{c}$ is a characteristic length often equal to the thickness or diameter of the body, and $k$ is the thermal conductivity of the body of interest. A very low Biot number $(B i<<1)$ indicates a relatively uniform temperature 
whereas a very high one $(B i \gg>1)$ indicates significant temperature variation within the copper.

Several different Biot analyses are necessary to fully characterize the copper ring. First, there are two different characteristic lengths: (1) one through the thickness of the copper and (2) one along a line running circumferentially around the copper ring. Likewise, there will be two different geometric Biot numbers, a radial one and a circumferential one. Also, there are two different heat transfer coefficients: (1) one on the hot side from the combustion products and (2) one on the cold side from the coolant. In this way, there will also be individual Biot numbers on the hot side and cold side. The four general Biot numbers that must be calculated to fully characterize the copper ring are then

(1) Radial hot side

(2) Radial cold side

(3) Circumferential hot side

(4) Circumferential cold side

The difficulty with finding the cold side Biot number is that the cold side heat transfer coefficient is unknown and in order to determine it, some assumptions must be made about the temperature distribution within the copper ring. In this way, the cold side heat transfer coefficient is a function of the Biot number and vice versa. Therefore, two distinct cases are analyzed for each cold side Biot number: (1) one in which the Biot number is assumed to be low, resulting in a constant copper temperature and (2) one in 
which the Biot number is assumed to be high, resulting in a temperature gradient within the copper. These two cold side heat transfer coefficients are then averaged to find an average cold side Biot number. If this value is significantly less than one, then it is likely the constant temperature assumption is more accurate but if it is significantly greater than one, the variable temperature assumption is likely more correct. The Biot cases considered are summarized in Figure 21. Note that steady state data collected in Runs 48 through 52 was used in the Biot analysis.

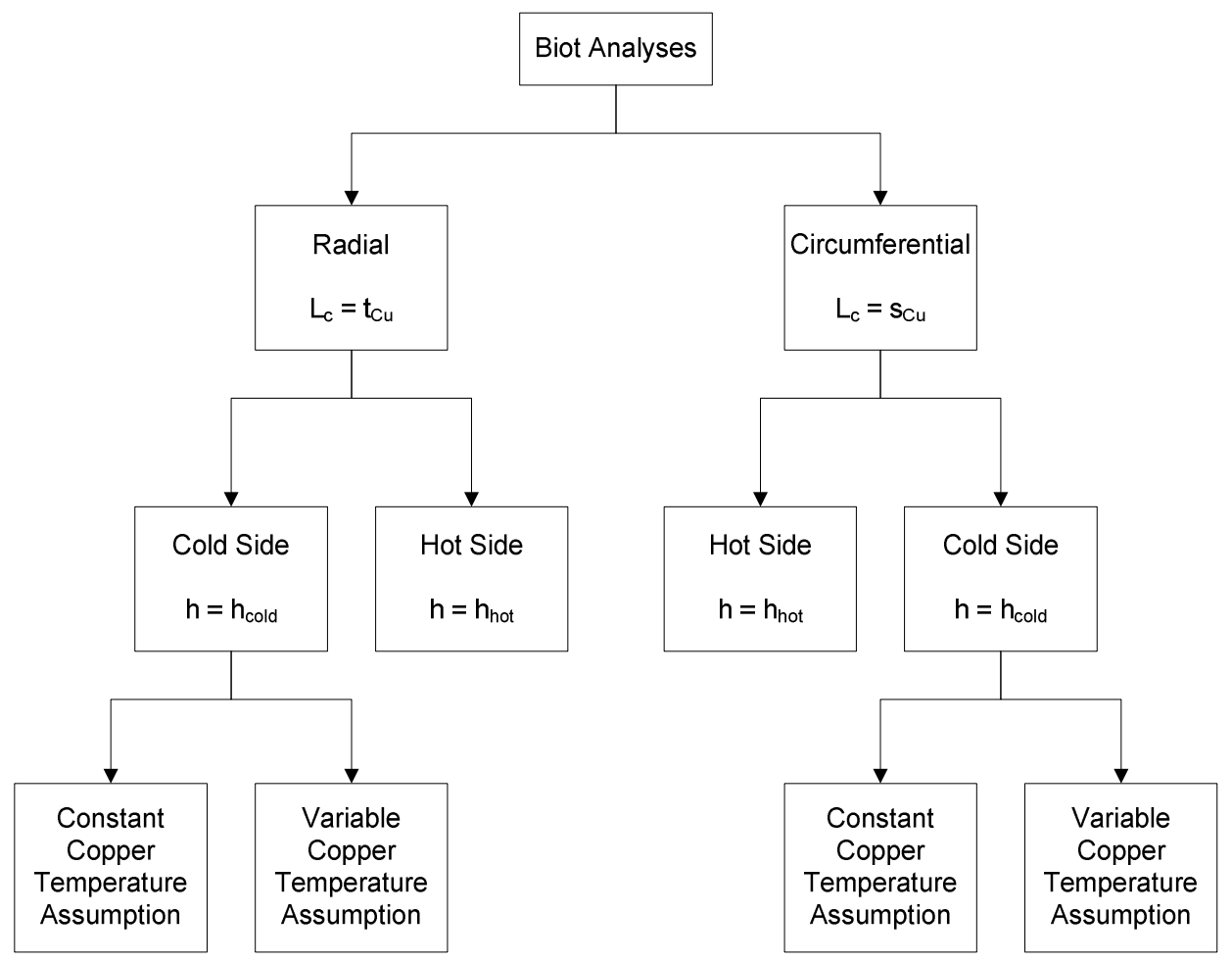

Figure 21. Biot analyses necessary to fully characterize copper ring

\section{II.3.2.1 Radial Biot Number}

For the radial Biot number analysis, the characteristic length is constant and defined as the ratio of the total cross sectional copper area to the mean copper thickness: 


$$
L_{c_{\text {radial }}}=\frac{A_{C u}}{\overline{t_{C u}}}
$$

The mean thickness of the copper is defined here by the average of the minimum and maximum thicknesses:

$$
\overline{t_{C u}}=\frac{t_{C u_{\max }}+t_{C u_{\min }}}{2}
$$

Note that the minimum thickness is the distance between points A and B in Figure 23 and the maximum thickness is the roughly distance between point $\mathrm{A}$ and $\mathrm{C}$ in Figure 23. The hot side radial Biot number is then given by

$$
B i_{\text {radial,hot }}=\frac{h_{\text {hot }} L_{c_{\text {radial }}}}{k_{C u}}
$$

where $h_{\text {hot }}$ is given by the Bartz equation. Because the cold side heat transfer coefficient is unknown, an energy balance with the hot side will be performed in two ways: first assuming a constant copper temperature and then assuming a temperature distribution within the copper. In both cases, the heat transfer is modeled as one-dimensional as shown in Figure 22. 


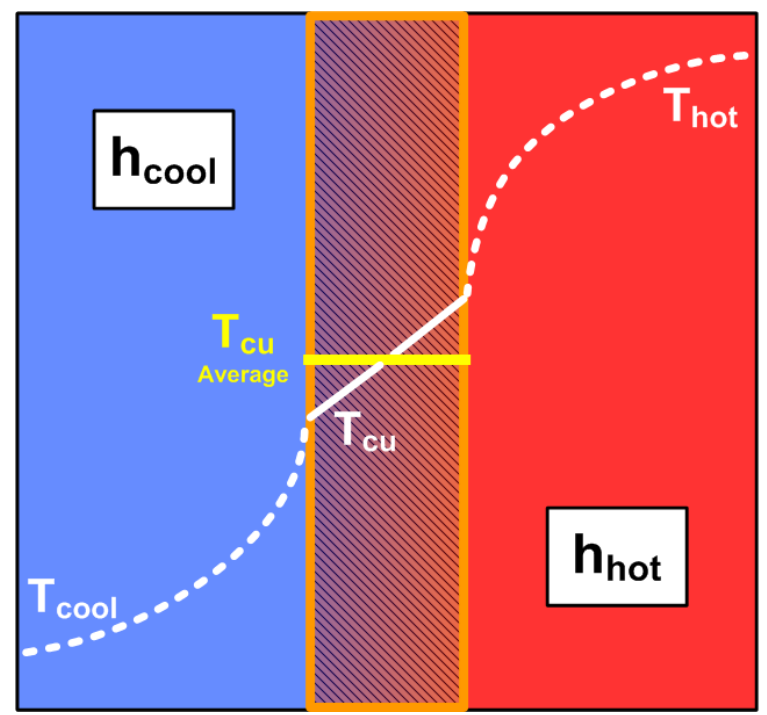

Figure 22. One-dimensional modeling of copper ring in radial Biot analyses (12)

For the constant temperature case, the copper temperature is given by an average of all of the measured steady state copper temperatures through the annulus. This is shown in Figure 23.

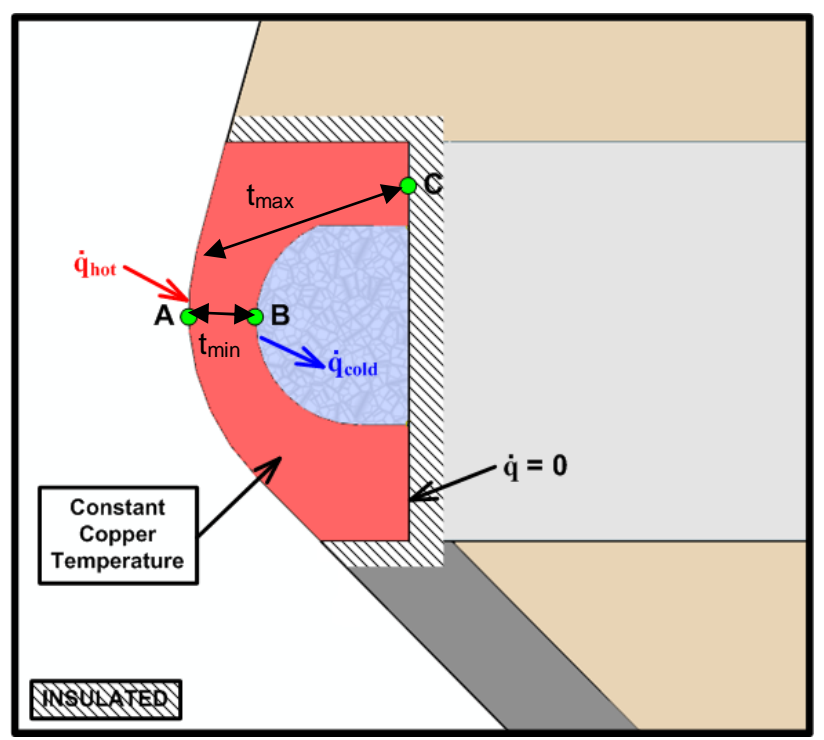

Figure 23. Low radial Biot number assumption (12) 
The heat transfer from the hot side is known and given by

$$
q_{\text {hot }}=h_{\text {hot }} A_{\text {hot }}\left(T_{\text {hot }}-T_{C u_{\text {ave }}}\right)
$$

By assuming the copper ring is insulated to all surroundings except the coolant and combustion gases, all of the heat from the hot side is convected out of the copper by the coolant giving

$$
q_{\text {cold }}=q_{\text {hot }}
$$

Similarly, an expression for the heat transfer to the coolant is

$$
q_{\text {cold }}=h_{\text {cold }} A_{\text {cold }}\left(T_{\text {Cu ave }}-T_{\text {cold }}\right)
$$

Equating equations (2.36) and (2.38) and solving for the cold side heat transfer coefficient gives

$$
h_{\text {cold }_{\text {min }}}=\frac{h_{\text {hot }} A_{\text {hot }}\left(T_{\text {hot }}-T_{C_{\text {ave }}}\right)}{A_{\text {cold }}\left(T_{C u}-T_{\text {cold }_{\text {ave }}}\right)}
$$

As it is desirable to predict the lowest physically possible cold side heat transfer coefficient, one additional assumption is made. In reality, the copper area exposed to the combustion gases is larger than that exposed to the coolant. In order to minimize the cold 
side heat transfer coefficient given by equation (2.39), only as much area on the cold side as there exists on the hot side was allowed to participate in the heat transfer process. In this way, the two areas were equated:

$$
A_{\text {hot }}=A_{\text {cold }}
$$

This assumption represents the bounding case in which the Biot number is an absolute minimum. Finally, the minimum cold side heat transfer coefficient is given by

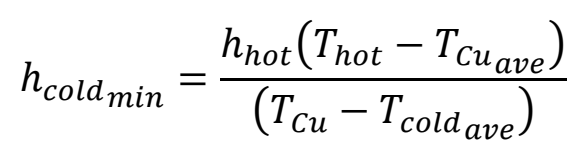

Because experimental data is available for both the coolant and copper temperatures from previous tests, all of the terms on the right side of equation (2.41) are known and the expression for the cold side heat transfer coefficient can be evaluated.

For the second case, the cold side Biot number is assumed high, signifying a temperature gradient within the copper. This is shown in Figure 24. 


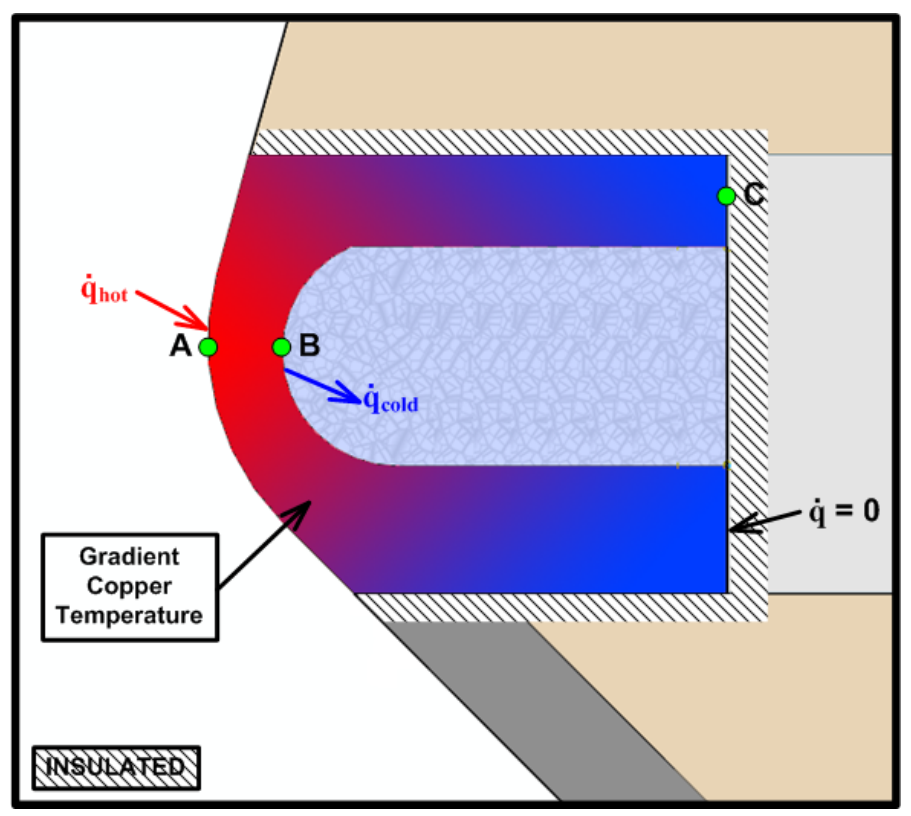

Figure 24. Schematic of high Biot number scenario (12)

As the geometry under investigation makes this case highly complex, several simplifying assumptions are necessary. Each assumption is conservatively chosen such that it increases the heat transfer coefficient rather than decrease it. In this way, despite the assumptions, the Biot number yielded will be the largest possible for this scenario. First, the hottest measured temperature in the copper ring $T_{C u \text {,max }}$ is assumed to occur along the surface closest to the combustion gases (at point A in Figure 24) and the coldest measured temperature $T_{C u \text {, min }}$ is assumed to occur along the edge closest to the coolant (point B in Figure 24). Combining these assumptions with Newton's Law of Cooling gives the following heat transfer rates on the hot and cold sides respectively: 


$$
\begin{gathered}
q_{\text {hot }}=h_{\text {hot }} A_{\text {hot }}\left(T_{\text {hot }}-T_{C u_{\max }}\right) \\
q_{\text {cold }}=h_{\text {cold }} A_{\text {cold }}\left(T_{C u_{\text {min }}}-T_{\text {cold }}\right)
\end{gathered}
$$

Again, all of the heat from the hot side is assumed to be transferred into the cold side. Therefore, equating equations (2.42) and (2.43) above and solving the for cold side heat transfer coefficient gives

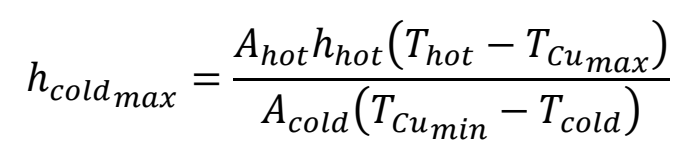

The average heat transfer coefficient for the high Biot number and cold Biot number assumption is given by

$$
h_{\text {cold }_{\text {ave }}}=\frac{h_{\text {cold }_{\max }}+h_{\text {cold }_{\text {min }}}}{2}
$$

Finally, the average cold side radial Biot number is given by

$$
B i_{\text {radial }, \text { cold }}=\frac{h_{\text {cold }_{\text {ave }} L_{c_{\text {radial }}}}}{k_{C u}}
$$

This analysis was performed for Runs 48 through 53. The resulting hot side radial Biot numbers are given in Figure 25. 


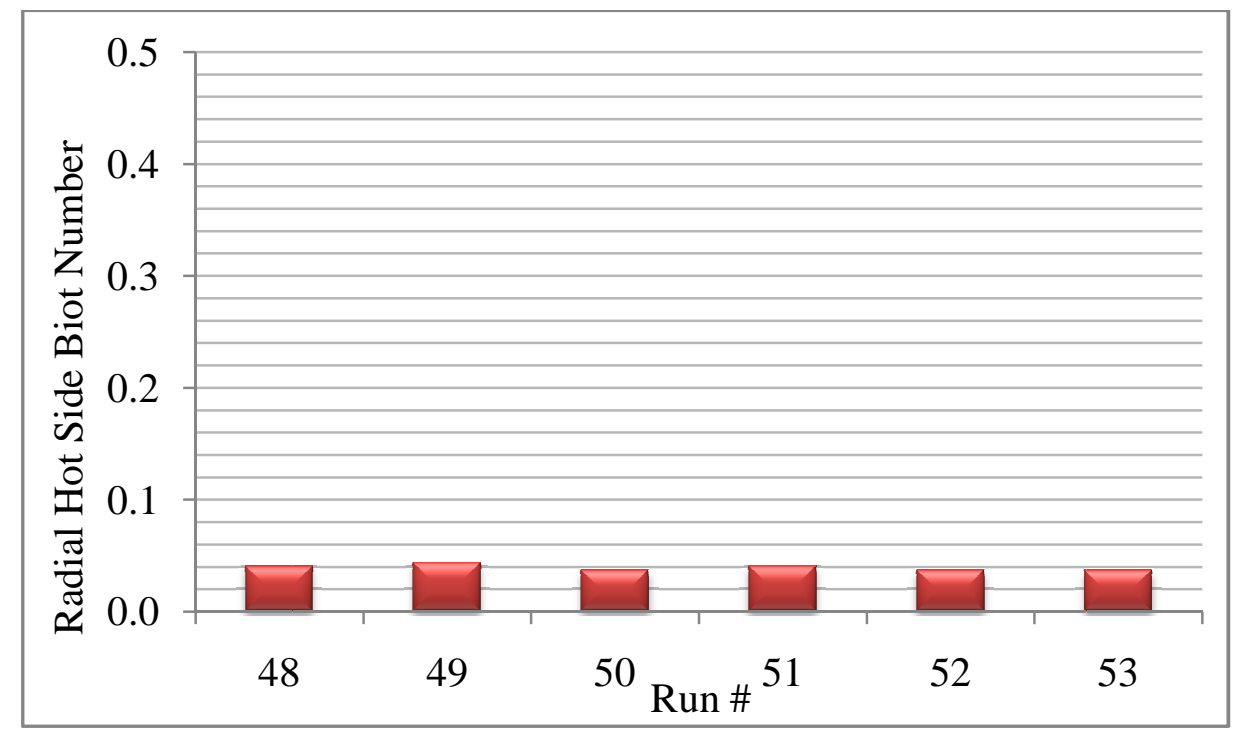

Figure 25. Radial hot side Biot numbers

The radial cold side Biot numbers are shown in Figure 26. The average value for all of the applicable runs is approximately 0.27 which is higher than the equivalent hot side value but still less than one indicating a stronger preference for the constant temperature scenario.

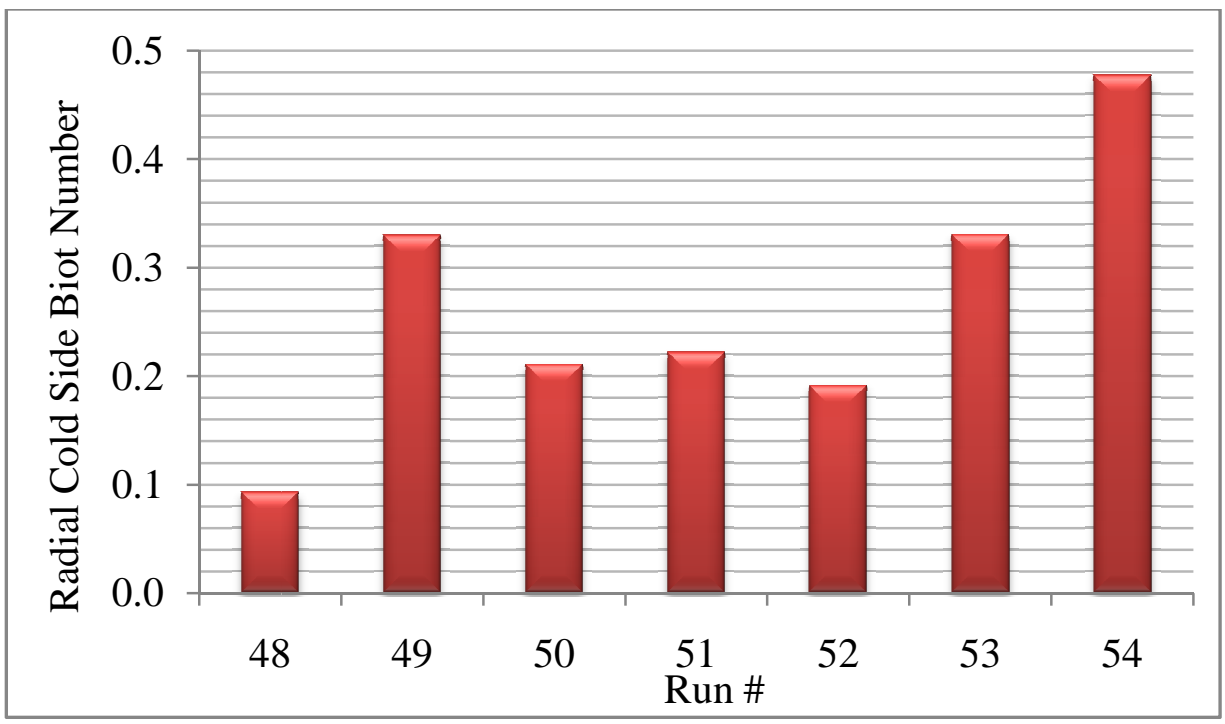

Figure 26. Radial cold side Biot numbers 
Based on the radial Biot results presented above, the temperature distribution through the thickness of the copper will be neglected. Typically, Biot numbers less than 0.01 are ideal for making this assumption which was not the case. Accordingly, there will likely be some error associated with assuming a constant copper temperature in the radial direction but the benefits of the simplifications this assumption allows outweigh the consequences of introducing this error.

\section{II.3.2.2 Circumferential Biot Number}

Runs 49 through 52 used the two flow-paths half-circle cooling annulus. Figure 27 shows a top down view of a two-flow paths nozzle.

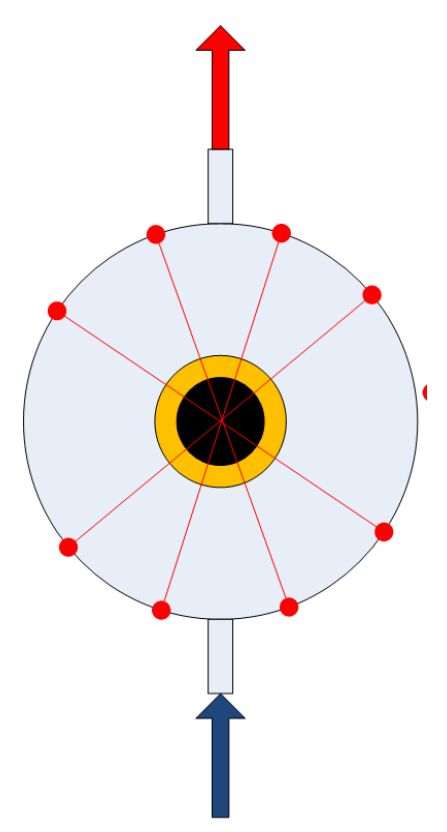

Figure 27. Two flow-paths nozzle top down schematic 
Each of the red dots indicates a port for measuring either coolant temperature, copper temperature or coolant pressure although not all of the ports are used the same way for each of the runs. The blue arrow indicates a coolant inlet and the red arrow indicates a coolant outlet. Five distinct sections are identified for analysis and are labeled 1 through 5 in Figure 27. The purpose of this analysis is to determine whether or not each of the sections can be modeled as having a constant temperature. A separate Biot analysis is therefore necessary for each section.

The flow and related hydrodynamic and thermal properties are assumed to be symmetrical about a vertical axis through the center of the nozzle. Making this assumption allows measurements taken from the ports on the right hemisphere to be used in conjunction with those taken on the left hemisphere which offers a complete set of data for calculations. Also by this symmetry the right hemisphere need not be analyzed. Notice that no temperatures are measured at the locations of the coolant inlet and outlet. These temperatures are necessary for analysis and are therefore linearly extrapolated from the temperature gradient between the two closest measurements.

Sections 1 and 5 ( $\pi / 10$ radians) are half the size of sections 2 and 4 ( $\pi / 5$ radians) and section $3(2 \pi / 5$ radians) is twice the size of sections 2 and 4 . The critical length for each section is equal to its average arc length $s_{\text {arc }}$ given by

$$
L_{c_{\text {circumferential }}}=s_{\text {arc }}=\frac{\Delta \theta}{2 \pi} \frac{V_{C u}}{A_{C u}}
$$


where $V_{C u}$ is the total volume of the copper ring, $A_{C u}$ is the cross sectional area of copper and $\Delta \theta$ is the angle spanned by the section of interest (i.e. $\pi / 10, \pi / 5$ or $2 \pi / 5$ radians). Because the characteristic length varies between each of the five sections, a separate hot side Biot number is calculated for each section. Using the same Bartz heat transfer coefficients from the radial Biot number analysis, hot side Biot numbers for each section are given by

$$
B i_{\text {circumferential,hot }}=\frac{h_{h o t} S_{a r c}}{k_{C u}}
$$

A separate cold side Biot number is also calculated for each of the five sections. As was the case for the radial Biot analysis, knowledge of the cold side heat transfer coefficient is necessary prior to calculating a cold side circumferential Biot number. Unfortunately, these two values are interrelated and cannot be found independently of each other. Therefore the same two cases that were analyzed in the radial Biot case will now be analyzed here: one in which the Biot number is assumed low and one in which it is not.

For the case in which the Biot number is assumed low, the copper temperature is constant. An average copper temperature is found for each section based on the two temperatures measured at the edges of that section. Each section is then analyzed using a finite difference approach based on conservation of energy. The four considered heat transfer terms for each section are: (1) convection in from the combustion gases, (2) convection out to the coolant, (3) conduction in from a hotter neighboring section and (4) conduction out to a colder neighboring section. Similar to the radial case, this analysis 
was performed in a one-dimensional manner. A representative diagram of this concept is shown in Figure 28. By symmetry, the edges of sections 1 and 5 not bordering another labeled section are assumed to be insulated.

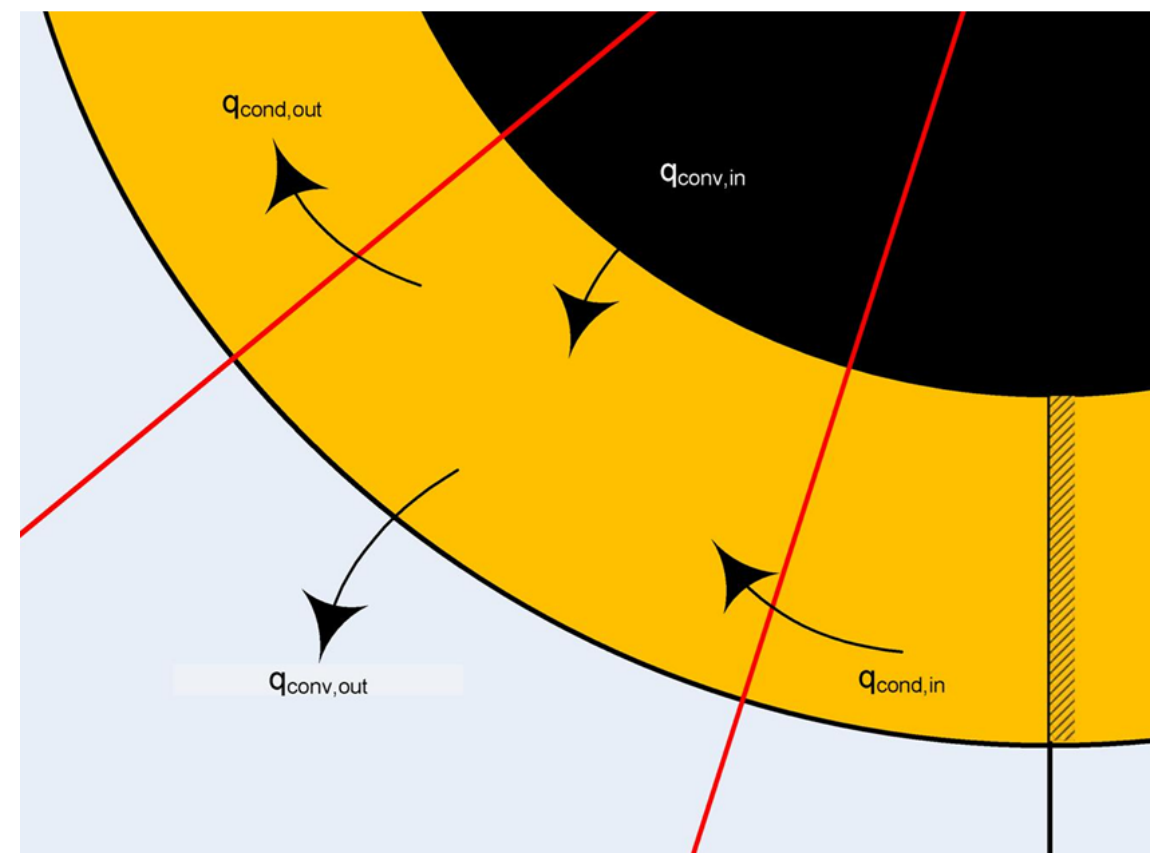

Figure 28. Circumferential Biot number control volume

The conduction heat transfer into the section of copper is governed by Fourier's Law which is given in polar coordinates by

$$
q_{\text {cond }, \text { in }}=\frac{k A_{C u}}{R} \frac{\partial T}{\partial \theta}
$$

Discretization of equation (2.49) gives 


$$
q_{\text {cond,in }} \approx \frac{k_{C u} A_{C u}}{R_{\text {ave }}} \frac{\Delta T}{\Delta \theta}=\frac{k_{C u} A_{C u}}{R_{\text {ave }}} \frac{T_{\text {nh }}-T_{\text {ave }}}{\Delta \theta_{\text {ave }, n h}}
$$

where $R_{\text {ave }}$ is the average radius of the copper cross section, $A_{C u}$ is the cross-sectional area of copper ring, $T_{n h}$ is the average copper temperature of hotter neighboring section, $T_{a v e}$ is the average copper temperature of section of interest, $k_{C u}$ is thermal conductivity of copper, and $\Delta \theta_{\text {ave,nh }}$ is the average angle spanned by current section and the section from which heat is conducting in. In equation form, $\Delta \theta_{\text {ave, } n h}$ is given by

$$
\Delta \theta_{\text {ave }, n h}=\frac{\Delta \theta+\Delta \theta_{n h}}{2}
$$

where $\Delta \theta$ is the arc angle of the current section and $\Delta \theta_{n h}$ is that of the hotter neighboring section. Similarly, the conduction out of this section is given by

$$
q_{\text {cond,out }} \approx \frac{k_{C u} A_{c S}}{R_{\text {ave }}} \frac{\Delta T}{\Delta \theta}=\frac{k_{C u} A_{C u}}{R_{\text {ave }}} \frac{T_{\text {ave }}-T_{n c}}{\Delta \theta_{\text {ave }, n c}}
$$

where $\Delta \theta_{\text {ave,nc }}$ is the average span angle of current section and the section to which heat is conducting. In equation form, $\Delta \theta_{a v e, n c}$ is given by

$$
\Delta \theta_{\text {ave }, n c}=\frac{\Delta \theta+\Delta \theta_{n c}}{2}
$$

The convective heat transfer into the copper is governed by Newton's Law of Cooling: 


$$
q_{c o n v, \text { in }}=\frac{\Delta \theta}{2 \pi} h_{\text {hot }} A_{\text {hot }}\left(T_{\text {hot }}-T_{\text {ave }}\right)
$$

where $A_{\text {hot }}$ is the total surface area of copper contacting hot gases, $T_{h o t}$ is the temperature of the combustion gases, $h_{h o t}$ is the heat transfer coefficient of the combustion gases, and $\Delta \theta$ is the arc length of current section. Similarly, the convective heat transfer out of the copper section is governed by

$$
q_{c o n v, o u t}=\frac{\Delta \theta}{2 \pi} h_{c o l d} A_{c o l d}\left(T_{a v e}-T_{c o l d}\right)
$$

where $A_{\text {cold }}$ is the total surface area of copper in contact with the coolant, $T_{\text {cold }}$ is the temperature of the coolant and $h_{\text {cold }}$ is the heat transfer coefficient of the coolant. Applying conservation of energy to the section of interest gives

$$
q_{\text {conv,out }}=q_{\text {conv,in }}+q_{c o n d, \text { in }}-q_{c o n d, o u t}
$$

Substituting equations (2.50), (2.52), (2.54) and (2.55) into equation (2.56) and solving for $h_{\text {cold }}$ gives:

$$
\begin{array}{r}
h_{\text {cold }}=\frac{2 \pi}{\Delta \theta} \frac{1}{A_{\text {cold }}\left(T_{\text {ave }}-T_{\text {cold }}\right)}\left[\frac{\Delta \theta}{2 \pi} h_{\text {hot }} A_{\text {hot }}\left(T_{\text {hot }}-T_{\text {ave }}\right)\right. \\
\left.+\frac{k A_{c s}}{R_{\text {ave }}} \frac{T_{n h}-T_{\text {ave }}}{\Delta \theta_{\text {ave }, n b}}-\frac{k A_{c s}}{R_{\text {ave }}} \frac{T_{\text {ave }}-T_{n c}}{\Delta \theta_{\text {ave }, n c}}\right]
\end{array}
$$


The above equation is solved for each of the five sections to yield a cold side heat transfer coefficient assuming a constant temperature distribution in each section.

For the case in which a temperature gradient is allowed to exist within each section, a linear temperature distribution is assumed. The half circle coolant path is split into 200 pieces. Within each of the five sections, temperatures are linearly interpolated to map temperatures onto each of the 200 nodes. A representative schematic of this concept is shown in Figure 29.

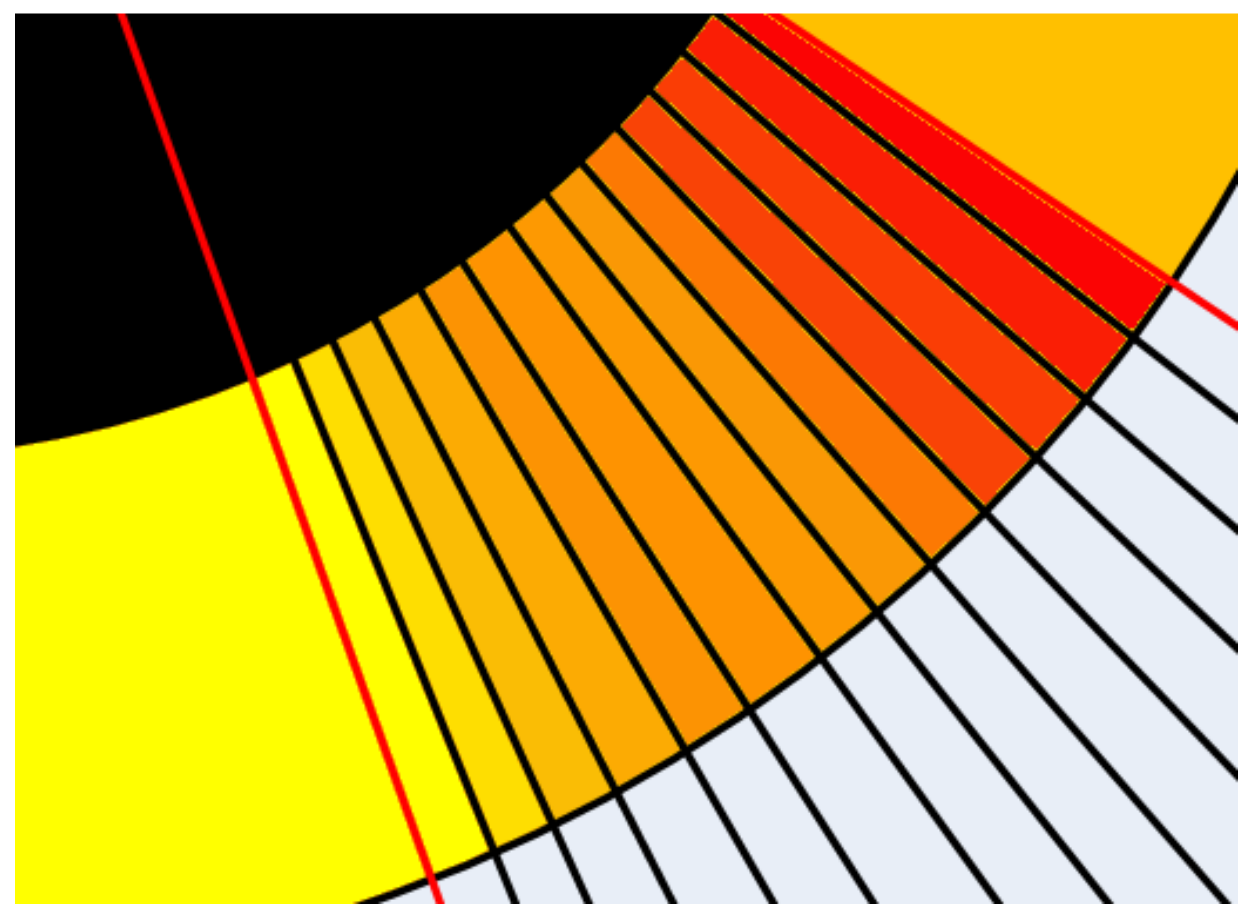

Figure 29. Variable copper temperature section

Finite difference equations are established for each of the smaller sections with conduction and convection equations very similar to those developed in the constant temperature case. Heat transfer coefficients for each of the small pieces are determined 
using equation (2.57). Those values are then averaged within each of the five initial sections to give average cold side heat transfer coefficients for each section. This is done for Runs 48 through 52 only. As was the case in the radial Biot number analysis, after cold side heat transfer coefficients for both scenarios (constant and variable copper temperature) had been determined, an average cold side Biot number is calculated for each section. Average cold side heat transfer coefficients for all applicable runs derived using the above method are shown in Figure 30 and tabulated values are given in Table 1.

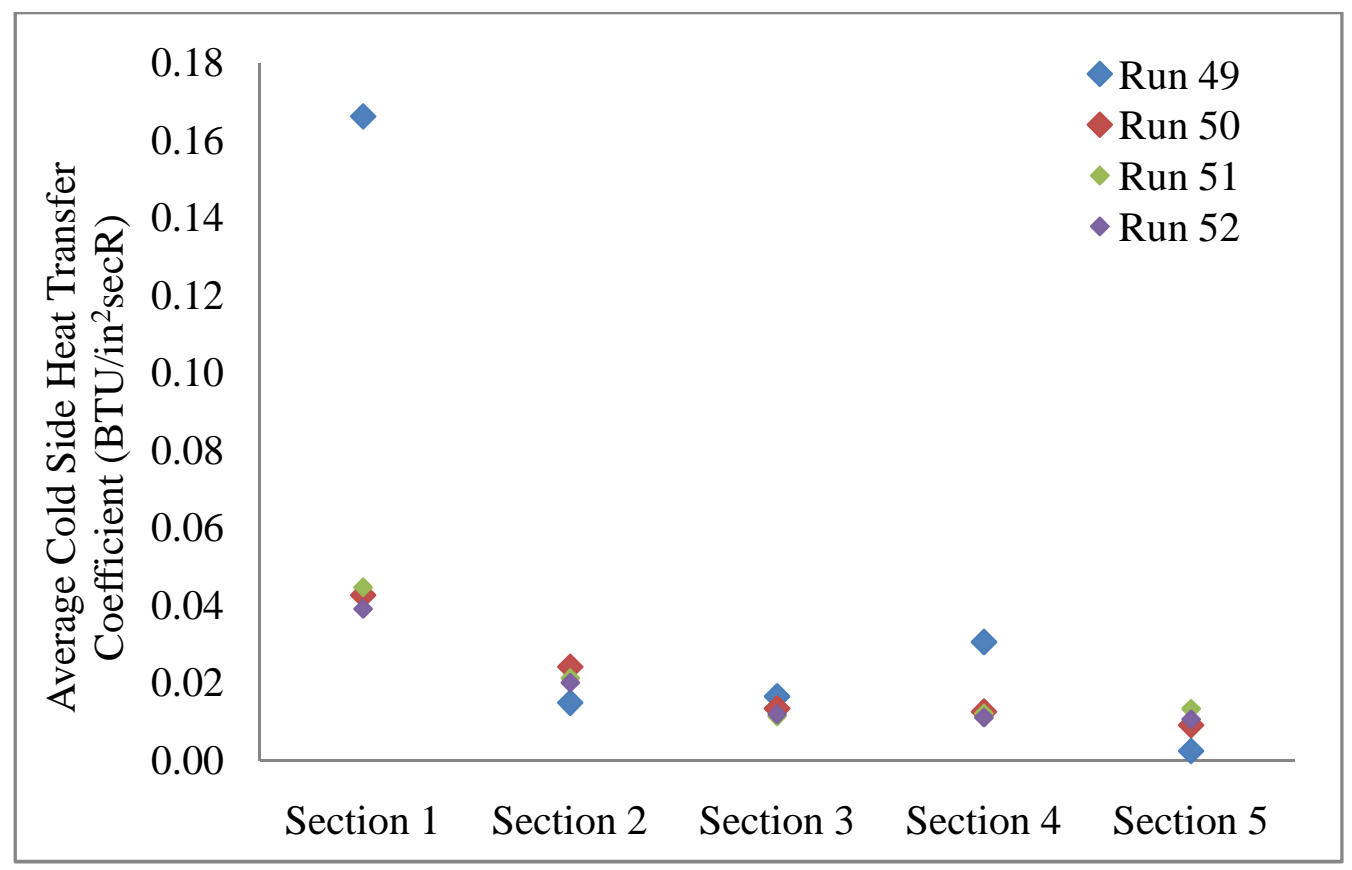

Figure 30. Experimental cold side heat transfer coefficients. 
Table 1. Experimental cold side heat transfer coefficients in BTU/in ${ }^{2}$ secR

\begin{tabular}{|c|c|c|c|c|c|}
\hline Section & Angle (degrees) & Run 49 & Run 50 & Run 51 & Run 52 \\
\hline 1 & 9 & 0.1280 & 0.0426 & 0.0480 & 0.0394 \\
\hline 2 & 36 & 0.0093 & 0.0240 & 0.0206 & 0.0199 \\
\hline 3 & 90 & 0.0179 & 0.0133 & 0.0106 & 0.0109 \\
\hline 4 & 144 & 0.0341 & 0.0124 & 0.0169 & 0.0161 \\
\hline 5 & 171 & 0.0021 & 0.0090 & 0.0132 & 0.0105 \\
\hline
\end{tabular}

Recall that the copper temperatures on the cold side of section 1 and the hot side of section 5 were not measured directly but rather linearly extrapolated from the two closest measurements. In Run 49, there was an inexplicably large gradient between the two temperatures that were used to determine the inlet temperature to section 1. It is likely that in this case the extrapolation method is not valid and accordingly, the heat transfer coefficient for section 1 or Run 49 should be considered an outlier.

Hot and cold side Biot numbers are shown by section in Figure 31 and Figure 32. See Table 2 for tabulated numerical values.

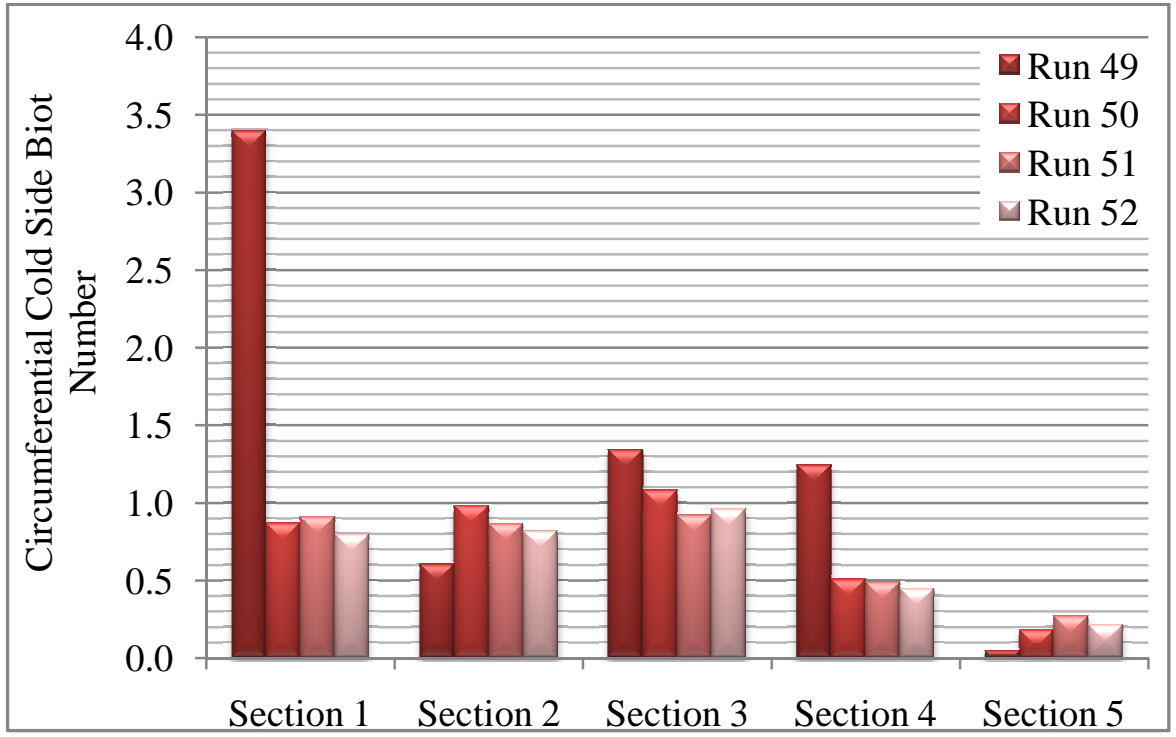

Figure 31. Circumferential cold side Biot numbers by section. 


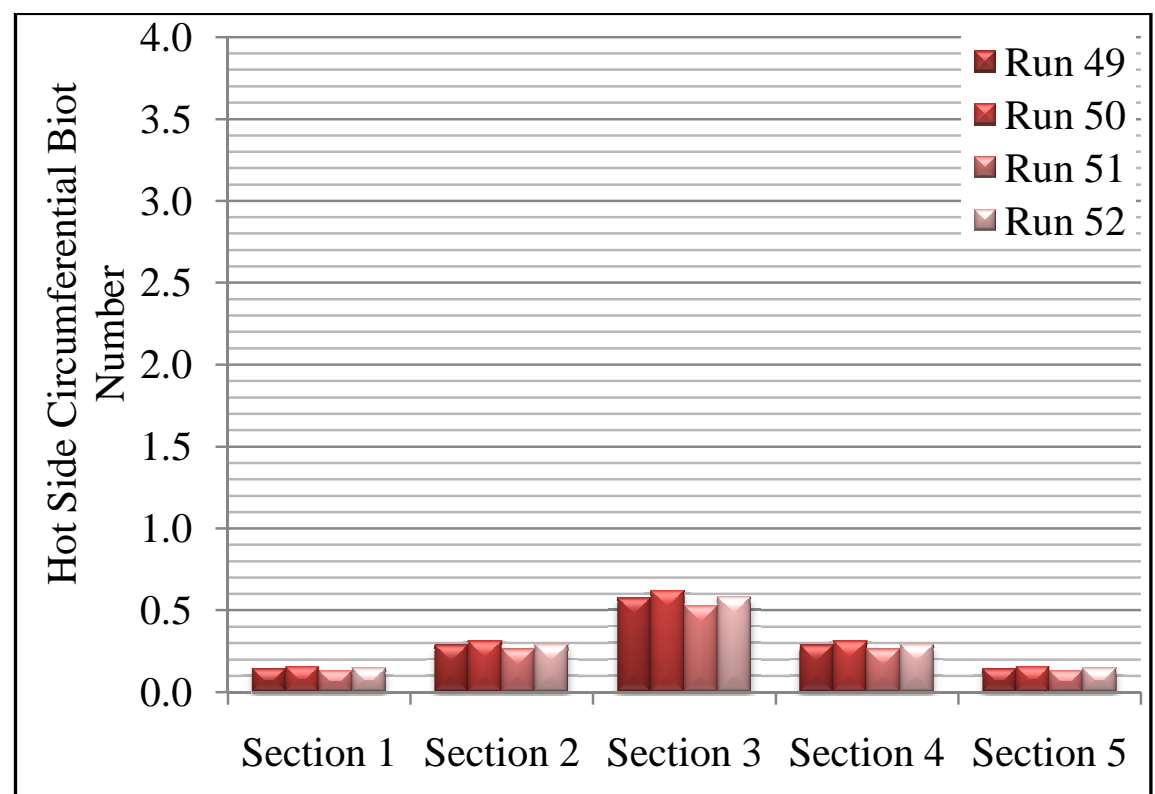

Figure 32. Circumferential hot side Biot numbers by section.

As was the case in the radial analysis, the cold side circumferential Biot numbers are significantly higher than those on the hot side. In most cases, the cold side values are only slightly lower than one. As was the case for the cold side heat transfer coefficient in section 1 of Run 49, the abnormally high Biot number is likely due to the method of determining the copper inlet temperature of that section. This data point will be considered an outlier. The overwhelming trend is still that the cold side circumferential Biot numbers are approaching unity which indicates that the copper temperatures are somewhere between the case in which the distribution can be neglected $(\mathrm{Bi} \ll<1)$ and that in which it cannot $(\mathrm{Bi}>>1)$. In the case of the hot side, the circumferential Biot numbers are less than one but not overwhelmingly so. Still, for the sake of simplicity in subsequent analyses, the copper temperatures within each section will be assumed constant. Table 2 below summarizes the minimum, maximum and average Biot numbers for each of the four cases considered. 
Table 2. Biot number analysis summary

\begin{tabular}{|r|c|c|c|c|c|}
\cline { 2 - 5 } & $\begin{array}{c}\text { Minimum } \\
\text { Biot } \\
\text { Number }\end{array}$ & $\begin{array}{c}\text { Maximum } \\
\text { Biot } \\
\text { Number }\end{array}$ & $\begin{array}{c}\text { Average } \\
\text { Biot } \\
\text { Number }\end{array}$ & $\begin{array}{c}\text { Significant } \\
\text { Temperature } \\
\text { Distribution? } \\
(\mathrm{Bi}>>1)\end{array}$ & $\begin{array}{c}\text { Constant } \\
\text { Copper } \\
\text { Temperature? } \\
(\mathrm{Bi}<<1)\end{array}$ \\
\hline Radial Hot Side & 0.037 & 0.044 & 0.040 & No & Yes \\
\hline Radial Cold Side & 0.093 & 0.330 & 0.209 & No & No \\
\hline Circumferential Hot Side & 0.132 & 0.620 & 0.283 & No & No \\
\hline Circumferential Cold Side & 0.048 & 3.397 & 0.849 & No & No \\
\hline
\end{tabular}

To reiterate, in all cases other than the radial hot side case, the Biot numbers are not low enough (i.e. less than 0.01) to merit neglecting temperature gradients within the copper ring. However, in the majority of cases, the calculated Biot numbers are less than one, indicating that the physical reality tended toward the constant temperature case rather than the variable temperate one. Based on this, the temperature of the copper insert will be assumed constant in subsequent analyses. It is noted that this assumption will likely introduce some error.

\section{II.4 FINAL RAYLEIGH MODELING ASSUMPTIONS}

Based on the discussion and analysis in the previous sections, the following assumptions will be uniform to all Rayleigh nozzle modeling:

1. The system operates at steady state.

2. Radiation heat transfer is negligible.

3. All of the heat from the combustion gases is transferred into the coolant. 
4. The copper temperature is uniform through the thickness of the copper and within each circumferential thermocouple section.

5. The combustion gas temperature and heat transfer coefficient are constant at their respective throat values.

6. The heat flux is constant over the length of the annulus and is determined using equation (2.12) with an average constant copper temperature.

7. Effects of bends in the copper annulus are negligible. This allows the annulus to be modeled as a straight pipe.

8. The cross-section of the annulus is modeled as circular with a diameter equal to its hydraulic diameter.

Based on these assumptions, the system will be modeled roughly as shown in Figure 33.

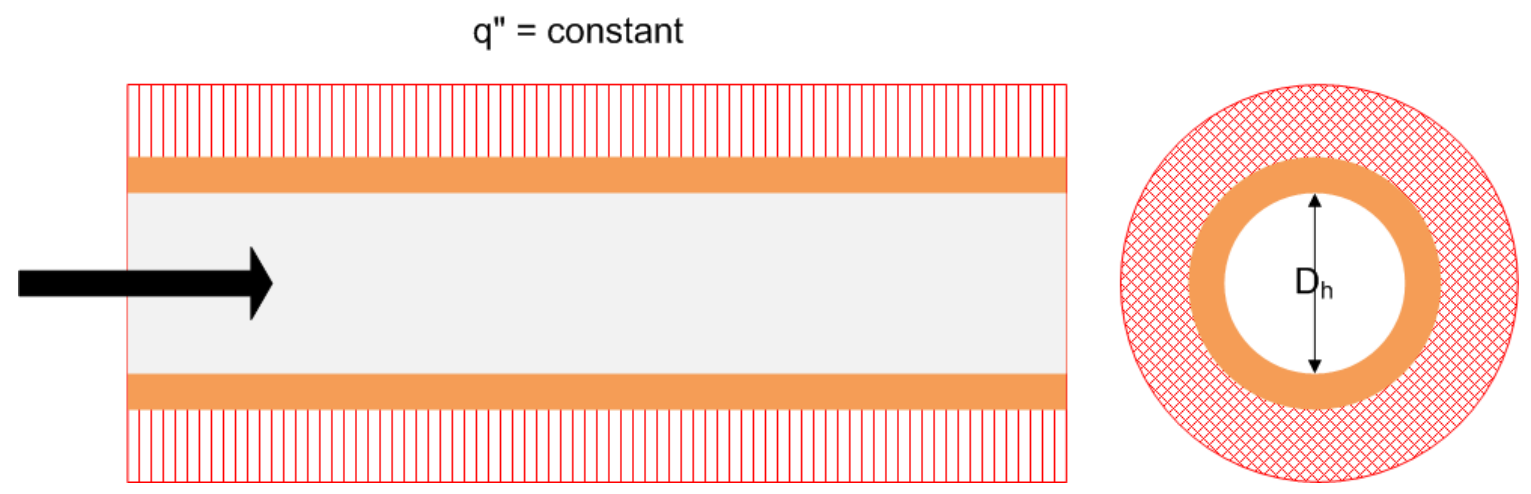

Figure 33. Simplified model of two-phase Rayleigh experiment 


\section{III: THE RAYLEIGH EXPERIMENT DESIGN AND PREPARATION}

The purpose of this section is to describe the preparation for gathering the data to validate a two-phase nitrous oxide model. Information regarding the instrumentation setup and equipment calibration is discussed.

\section{III.1 INSTRUMENTATION}

A brief discussion of the instrumentation used for data collection will be useful. The following sections include descriptions of the modifications to cooling system geometry in the Rayleigh nozzle as well as specifications of instrumentation used.

\section{III.1.1 Modifications to Nozzle Geometry}

In the previous versions of the Cal Poly cooled hybrid rocket nozzle, the coolant annulus included a single inlet and a single outlet with a half-circle coolant path. With this configuration, a total of eight ports were available for temperature and pressure monitoring. Because those experiments were not aimed solely at monitoring the coolant flow, the available instrumentation was sufficient. However, in order to validate a comprehensive two-phase coolant model, the instrumentation used in the Rayleigh experiments must be more thorough. To accommodate this need, several changes were made to the cooled nozzle geometry. Those changes are discussed below.

The most significant difference between previous nozzles and the Rayleigh nozzle is the flow path geometry. Instead of a split flow path around the throat of the nozzle, the coolant in the Rayleigh nozzle makes one full loop around the throat. This is 
advantageous for several reasons. First, more instrumentation can be used along the flow path, enabling more data to be collected. Second, the longer annulus gives the possibility of dumping more heat into the coolant than was the case with the previous nozzles. This may be desirable for validating a dryout model. Finally, removing the split in the flow path makes the system more amenable to being modeled as a one-dimensional system. This change is depicted in Figure 34.

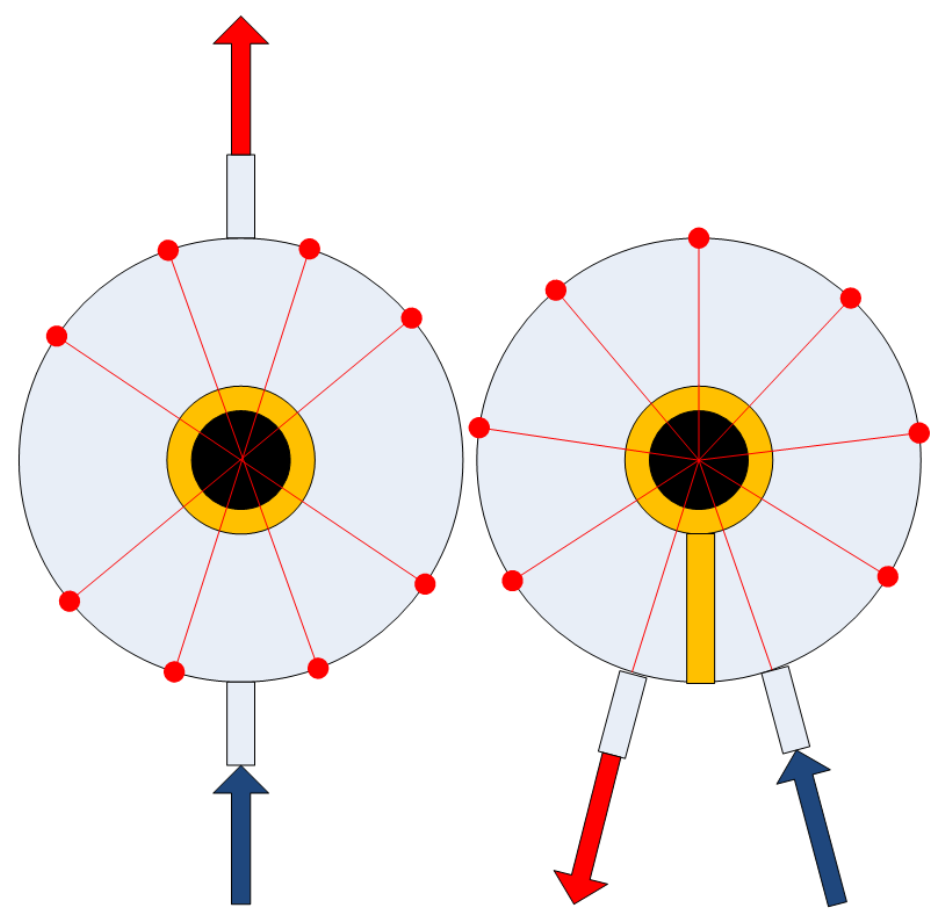

Figure 34. Modifications from the two flow-paths (left) to the Rayleigh nozzles (right)

Additional modifications were made including alignment of the copper and gas temperature thermocouples with the pressure transducer ports. The Rayleigh nozzle includes a total of nine instrumentation ports that are equally spaced circumferentially around the coolant annulus. Two are used for the coolant inlet and outlet. At the remaining seven locations (denoted by red dots in Figure 34), copper temperature, 
coolant temperature and coolant pressure are all monitored. Note that this is far superior to the two flow-paths geometry as only one of the three could be monitored at each instrumentation location in that case. In all, this yields twenty one total instrumentation ports in the Rayleigh nozzle as compared to eight offered in the previous nozzle geometry. Finally, while not entirely necessary, the equal spacing between instrumentation ports makes post-processing of the data much simpler than was previously the case. Figure 35 shows a three-dimensional rendering of the final Rayleigh nozzle:

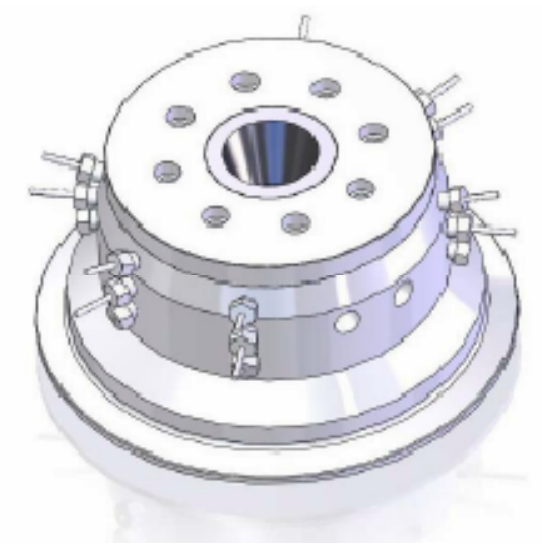

Figure 35. Rayleigh nozzle rendering

Figure 36 shows the location of the two temperature monitors and pressure monitor at each circumferential monitoring location. Prior to performing any tests, each of the copper and gas thermocouples were externally calibrated over a range of temperatures. The pressure transducers were also calibrated individually prior to testing. 


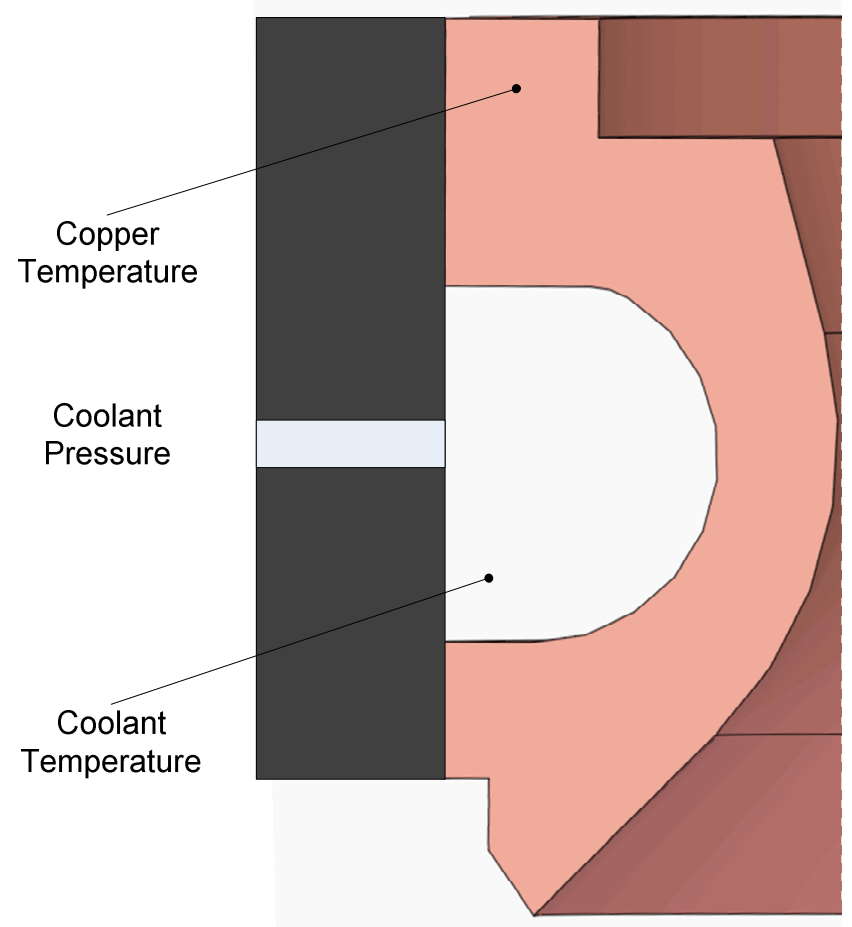

Figure 36. Location of coolant pressure transducer and coolant/copper thermocouples

The reported accuracy of the pressure transducers is \pm 2.5 psia (or $\pm 0.25 \%$ of the full scale reading). The thermocouples were reported accurate within $\pm 3^{\circ} \mathrm{F}$.

\section{III.2 EQUIPMENT VERIFICATION WITH A PERFECT GAS}

In an attempt to verify the Rayleigh nozzle setup and instrumentation, an experiment separate from those in which nitrous oxide is used as a coolant was proposed. In this experiment, a perfect gas would replace the nitrous oxide as the coolant, eliminating many of the complexities of the coolant flow modeling. The goal of this experiment and analysis is to demonstrate that a simple analytical model utilizing standard gas dynamics and heat transfer principles can predict the experimental behavior of the perfect coolant 
gas. This will ensure proper setup of the Rayleigh experiment and promote confidence in the data collected with the Rayleigh test equipment in subsequent experiments.

A perfect gas is defined as one in which the specific heats remain constant at all temperatures and pressures and the ideal gas constitutive law applies. In general, the cooling capacity of a perfect gas is less than that of a two-phase fluid. Accordingly, preliminary analysis is necessary to ensure that the wall temperatures in the Rayleigh nozzle do not exceed a "safe" wall temperature. As was discussed in previous chapters, a safe wall temperature is chosen to be $500^{\circ} \mathrm{F}$ which is the temperature above which a significant loss of yield strength is experienced by the copper insert. This value is used throughout the remainder of this analysis as a reference point. Descriptions of the experimental design process and setup as well as development of the analytical model follow.

\section{III.2.1 Experimental Design}

The expected cooling capacity of a single phase perfect gas is, in general, significantly smaller than that of two-phase nitrous oxide because it does not experience a phase change. As safety and preservation of the Rayleigh nozzle are of primary importance in these tests, combustion ("hot side") conditions were set such that the copper temperature does not exceed $500^{\circ} \mathrm{F}$. In the combustion process, the amount of fuel is fixed since the combustion chamber is tailored to a specific fuel grain geometry. The primary variable that can be controlled is the mass flow rate of oxidizer into the combustion chamber. This affects the firing process in two ways. First, it affects chamber pressures. A lower 
oxidizer mass flow rate yields less oxidizer in the fixed volume chamber and therefore a lower chamber pressure. Second, the oxidizer to fuel ratio $(\mathrm{O} / \mathrm{F})$ is affected by the oxidizer mass flow rate. A lower oxidizer mass flow rate decreases the $\mathrm{O} / \mathrm{F}$ since the amount of fuel is fixed in the chamber.

The chamber pressure and $\mathrm{O} / \mathrm{F}$ contribute significantly to the heat transfer coefficients and combustion gas temperatures in the throat of the nozzle. In general, a higher $\mathrm{O} / \mathrm{F}$ and chamber pressures will yield hotter combustion gases up to the stoichiometric ratio. The same trend can be seen for convective heat transfer coefficients as predicted by the Bartz equation (see Section II.3.1). Determining the optimum achievable $\mathrm{O} / \mathrm{F}$ and chamber pressure for the perfect gas experiment is not a simple task as the two variables are linked but a definite relationship between the two is not well established. In a standard test with nitrous oxide coolant at Cal Poly, typical operating conditions are a chamber pressure of $200 \mathrm{psi}$ and an $\mathrm{O} / \mathrm{F}$ of 2.0. A scatter plot showing the general trend of $\mathrm{O} / \mathrm{F}$ and chamber pressure for all recorded fires with Plexiglas ${ }^{\circledR}$ as fuel at Cal Poly is shown in Figure 37. While the relationship is weak, there appears to be a positive correlation between $\mathrm{O} / \mathrm{F}$ and chamber pressure. Note that this includes runs with a wide range of fuel grain geometries. The correlation would presumably be stronger for only short single grain runs. In any case, the positive correlation between $\mathrm{O} / \mathrm{F}$ and chamber pressure makes sense intuitively as an increase in oxidizer flow rate should cause an increase in both the $\mathrm{O} / \mathrm{F}$ and the chamber pressure. 


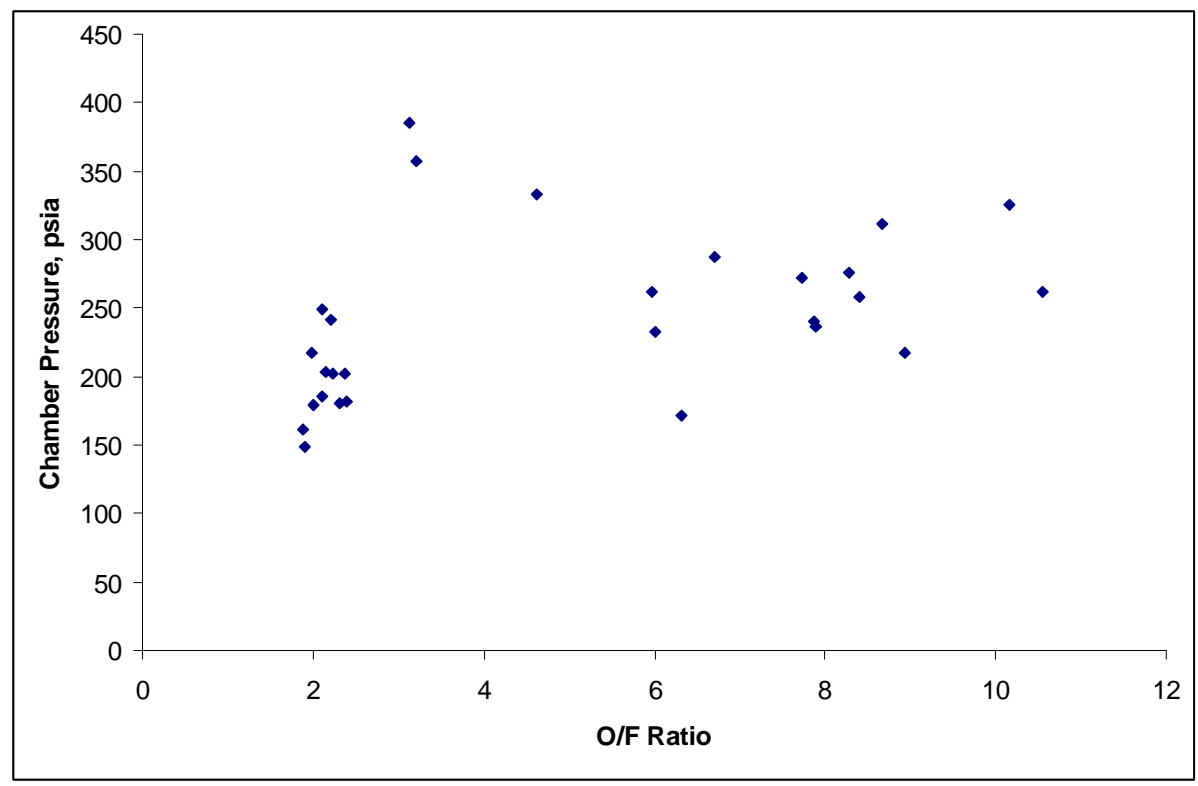

Figure 37. Previous firing conditions for all recorded Plexiglas ${ }^{\circledR} / \mathrm{N}_{2} \mathrm{O}$ tests at Cal Poly

To promote safety and equipment preservation, the lowest achievable chamber pressure was to be used for the ideal gas Rayleigh experiments. As these conditions were not known offhand at the time of the preliminary ideal gas experimental design, values were approximated. The lowest chamber pressure used in previous tests was originally thought to be approximately 70 psia. From the above plot, it is likely that for this chamber pressure, the $\mathrm{O} / \mathrm{F}$ will be lower than 2.0. However, because this value could not be approximated with any level of certainty, an $\mathrm{O} / \mathrm{F}$ of 2.0 was maintained. Again, these approximate values were only used in coolant selection. After running the test, the actual $\mathrm{O} / \mathrm{F}$ and chamber pressure were used as inputs in the coolant model. 


\section{III.2.1.1 Combustion ("Hot Side”) Analysis}

A chamber pressure of $70 \mathrm{psia}$ and an $\mathrm{O} / \mathrm{F}$ of 2.0 were input into TEP to determine characteristics of the combustion gases through the nozzle. From this, the gas temperature at the throat is estimated at $2971^{\circ} \mathrm{F}$. Additional properties from the TEP are input into the Bartz equation to yield an approximate heat transfer coefficient at the throat of $1.28 \mathrm{E}-3$

$\mathrm{BTU} / \mathrm{in}^{2} \mathrm{sec}^{\circ} \mathrm{R}$. The combustion gas temperature and heat transfer coefficient are used to determine the hot side heat transfer from the combustion gases using equation (3.1) below where $A_{\text {hot }}$ is the area of copper exposed to the hot side gases and is determined to be 0.7046 in $^{2}$ from solid modeling software.

$$
q=h_{\text {hot }} A_{\text {hot }}\left(T_{\text {hot }}-T_{C u}\right)
$$

The above equation yields a heat transfer rate of $2.23 \mathrm{BTU} / \mathrm{sec}$ from the combustion gases into the copper. Note that a constant copper wall temperature of $500^{\circ} \mathrm{F}$ is assumed in this portion of the analysis. The validity of this assumption is assessed later in the analysis. This approximate heat transfer value is then used as an input into the analysis of the cold side of the nozzle.

\section{III.2.1.2 Coolant ("Cold Side”) Suitability Analysis}

It is first necessary to find a perfect gas capable of handling the applied heat load of approximately $2.23 \mathrm{BTU} / \mathrm{sec}$. Then, a Rayleigh model of the coolant gas as it flows through the annulus is developed to yield copper and gas temperatures and gas pressure as a function of distance through the annulus. 
The goal of this portion of the analysis is to find a perfect gas capable of removing the applied heat flux with a reasonable mass flow rate. The perfect gases considered are: Hydrogen, Helium, Neon, Argon, Xenon and Krypton.

For simplicity, it is necessary to assume that the flow is thermally and hydrodynamically fully developed. For the case of a constant heat flux, this means that the heat transfer coefficient and difference between the wall and coolant temperatures are constant through the annulus. While making this assumption may contribute to a significant amount of error, it is conservative as it underestimates the average heat transfer coefficient since heat transfer coefficients are higher in entrance regions. A general rule of thumb for turbulent flows is the ratio of entrance length (hydrodynamic and thermal) to diameter is approximately 10 (16). For the geometry under investigation in the Rayleigh experiments (hydraulic diameter of approximately 0.1295 inches and annulus length of approximately 2.5 inches), this corresponds to an entrance region encompassing approximately $50 \%$ of the length of the annulus.

A common Nusselt number analogy method is used to determine the cold side heat transfer coefficient for each of the gases considered. This method is based on the following basic relation

$$
N u=\frac{h D}{k}=S t \cdot R e \cdot P r
$$


where $R e$ is the Reynolds number, $P r$ is the Prandtl number and $S t$ is the Stanton number. The Reynolds and Prandtl numbers are given by:

$$
R e=\frac{u D}{v}=\frac{\dot{m D}}{A_{c s} \mu}
$$

and

$$
\operatorname{Pr}=\frac{\mu C_{p}}{k}
$$

where $u$ is the gas velocity, $D$ is the diameter of the annulus, $v$ is the gas kinematic viscosity, $\dot{m}$ is the mass flow rate of the gas, $A_{c s}$ is the cross sectional area of the annulus, $\mu$ is the molecular viscosity of the gas, $C_{p}$ is the gas specific heat and $k$ is the thermal conductivity of the gas. In this case the hydraulic diameter, $D_{H}$ is used in place of $D$ as the annulus is not circular. The Stanton number is calculated using the Petukhov correlation as shown below (16):

$$
S t=\frac{\frac{C_{f}}{2}}{1.07+12.7\left(\operatorname{Pr}^{2 / 3}-1\right)\left(\frac{C_{f}}{2}\right)^{1 / 2}}
$$

In the above relation, $C_{f}$ is the friction factor and is given by

$$
C_{f}=2(2.236 \ln (R e)-4.639)^{-2}
$$


Note that this correlation is reported accurate for Reynolds numbers between 1E4 and 5E6 and Prandtl numbers between 0.5 and 2000.

Using the above correlation, heat transfer coefficients for each of the considered coolant gases are simply a function of mass flow rate. The strategy of this analysis is to parametrically modify the mass flow rate for each of the considered gases until the calculated heat transfer rate is greater than the heat transfer rate from the hot side. This ensures that the gas will offer sufficient cooling capacity to remove all of the heat added to the copper by the hot combustion gases. The results of this analysis are summarized in Table 3 along with individual material properties of each of the considered gases. Note that the Reynolds numbers for Xenon, Krypton and Argon are outside of the applicable range for the Petukhov correlation. Because of this, the results for these three gases are likely flawed and should not be used.

Table 3. Perfect gas cooling capacity analysis and physical properties summary

\begin{tabular}{|c|c|c|c|c|c|c|c|}
\cline { 2 - 8 } \multicolumn{1}{c|}{} & $\begin{array}{c}\mathbf{C p} \\
\left(\mathbf{B T U} / \mathbf{l b m}^{*} \mathbf{R}\right)\end{array}$ & $\begin{array}{c}\boldsymbol{\mu} \\
\left(\mathbf{l b m} / \mathbf{f t}^{*} \mathbf{s e c}\right)\end{array}$ & $\begin{array}{c}\mathbf{k} \\
\left(\mathbf{W} / \mathbf{m}^{*} \mathbf{K}\right)\end{array}$ & $\mathbf{P r}$ & $\mathbf{R e}$ & $\begin{array}{c}\mathbf{m} \\
(\mathbf{l b m} / \mathbf{s e c})\end{array}$ & $\begin{array}{c}\mathbf{q} \\
(\mathbf{B T U} / \mathbf{s e c})\end{array}$ \\
\hline Xenon & 0.039 & $1.54 \mathrm{E}-05$ & 0.0056 & 0.675 & $2.50 \mathrm{E}+07$ & 4.50 & 2.23 \\
\hline Krypton & 0.060 & $1.68 \mathrm{E}-05$ & 0.0093 & 0.676 & $1.50 \mathrm{E}+07$ & 2.80 & 2.29 \\
\hline Argon & 0.125 & $1.50 \mathrm{E}-05$ & 0.0175 & 0.670 & $7.00 \mathrm{E}+06$ & 1.20 & 2.28 \\
\hline Neon & 0.246 & $2.07 \mathrm{E}-05$ & 0.0477 & 0.665 & $2.10 \mathrm{E}+06$ & 0.50 & 2.24 \\
\hline Helium & 1.241 & $1.32 \mathrm{E}-05$ & 0.1541 & 0.662 & $5.30 \mathrm{E}+05$ & 0.08 & 2.30 \\
\hline Hydrogen & 3.417 & $5.94 \mathrm{E}-06$ & 0.1832 & 0.690 & $4.40 \mathrm{E}+05$ & 0.03 & 2.43 \\
\hline
\end{tabular}

Based on the required flow rates given in Table 3, helium and hydrogen are deemed the most reasonable gases for this test. Note that the physical properties are evaluated at 20 degrees Celsius as a first approximation. The mass flow rates required to provide 
sufficient heat transfer rates are significantly lower than the remaining four gases. Helium is chosen for first consideration over hydrogen based on the safety concerns of hydrogen (flammability, etc.). However, if for any reason helium is not able to fulfill the requirements of the test, hydrogen is a reasonable second choice as it is often used in liquid rocket cooling.

\section{III.2.2 Helium Perfect Gas Modeling}

A Rayleigh model of the helium as it flows through the coolant annulus is presented here. The Rayleigh nozzle is equipped to monitor pressure, gas temperature and copper temperature at seven ports covering the full span of the annulus. Accordingly, the

primary aim of the analytical model is to predict the gas pressure, gas temperature and copper temperature at discrete points along the annulus. The method of determining those values is summarized below in the following sections.

\section{III.2.2.1Model Inputs and Problem Description}

The inputs necessary to run this model are: (1) O/F, (2) chamber pressure, (3) mass flow rate, (4) inlet annulus temperature, and (5) inlet annulus pressure. Note that once the $\mathrm{O} / \mathrm{F}$ and chamber pressure are input, the coolant heat transfer coefficient is reevaluated using equations (3.2) through (3.6).

Based on the above inputs, the inlet density is calculated from the ideal gas law and the known inlet temperature and pressure. This gives 


$$
\rho_{\text {in }}=\frac{P_{\text {in }}}{R T_{i n}}
$$

where $R$ is the specific gas constant for helium. The inlet velocity is calculated from continuity:

$$
u_{i n}=\frac{\dot{m}}{\rho_{i n} A_{c s}}
$$

where $A_{c s}$ is the cross sectional area of the annulus. The speed of sound at the inlet is given by

$$
c_{\text {in }}=\sqrt{\gamma R T_{\text {in }}}
$$

where $\gamma$ is the specific heat ratio of Helium which is a known quantity. The inlet Mach number can then be calculated using the equation below:

$$
M_{i n}=\frac{u_{i n}}{c_{\text {in }}}
$$

The stagnation temperature at the inlet is calculated by:

$$
T_{o, \text { in }}=T_{\text {in }}\left(1+\frac{\gamma-1}{2} M_{\text {in }}^{2}\right)
$$




\section{III.2.2.2 Gas Temperature Distribution}

The stagnation temperature at any axial location, $z$ in the annulus can be determined using the following function derived from conservation of energy:

$$
T_{o, z}=T_{o, i n}+\frac{Q_{i n-z}}{C_{p}}
$$

where $Q_{i n-z}$ is the heat transfer per unit mass applied to the annulus between the inlet and location $z$ and can be calculated by

$$
Q_{i n-z}=\frac{q_{i n-z}}{\dot{m}}
$$

where $q_{i n-z}$ is the heat transfer rate (in BTU/sec) applied to the flow between the inlet and location $z$ and is calculated by multiplying the total heat transfer rate by the ratio of distance from the inlet to location $z$ to the total length of the annulus $L$. In equation form, this gives

$$
q_{i n-z}=\left(\frac{Z}{L}\right) q
$$

where $q$ is the total heat transfer rate from the hot side analysis. Note that using this value of $q$ implicitly assumes that the copper temperature is constant at $500^{\circ} \mathrm{F}$ and that all of the heat from the combustion gases is transferred into the coolant gases. The validity of this assumption will be discussed later. 
The ratio of the stagnation temperatures at point $z$ and the inlet is a function of the Mach numbers at those locations only. Therefore, since the stagnation temperatures at both points as well as the inlet Mach number are known, the Mach number at location $z$ can be found using the following function (2):

$$
\frac{T_{o, z}}{T_{o, i n}}=\frac{M_{z}^{2}}{{M_{i n}}^{2}} \frac{\left(1+\gamma{M_{i n}}^{2}\right)^{2}}{\left(1+\gamma M_{z}^{2}\right)^{2}}\left(\frac{1+\frac{\gamma-1}{2} M_{z}^{2}}{1+\frac{\gamma-1}{2} M_{i n}^{2}}\right)
$$

Because this equation cannot be solved algebraically for $M_{z}$, MATLAB is used to iteratively solve for $M_{z}$ at each location $z$ in the annulus. Knowledge of the Mach number at each point in the annulus allows the static temperature to be calculated using the following equation:

$$
T_{z}=\frac{T_{o, z}}{1+\frac{\gamma-1}{2} M_{z}{ }^{2}}
$$

\section{III.2.2.3 Copper Temperature Distribution}

To find the copper temperature, a simplified energy balance method is used. Assuming all of the heat from the combustion gases is transferred into the coolant, the difference between the coolant and copper temperature at any location $z$ in the annulus is constant and given by 


$$
T_{C u, z}-T_{z}=\frac{q}{h_{\text {cold }} A_{\text {cold }}}
$$

where $h_{\text {cold }}$ is the coolant heat transfer coefficient, $q$ is the heat transfer rate found from the hot side analysis, and $A_{\text {cold }}$ is the area of copper exposed to the coolant. Recall from the coolant suitability analysis that a Nusselt number correlation was used to find $h_{\text {cold }}$ for helium.

After finding the copper temperature distribution, the constant temperature assumption made when analyzing the hot side must be revisited. To find the heat transfer into the cold side, it was initially assumed that the copper temperature was constant at $500^{\circ} \mathrm{F}$. Equation (3.17) above yields an updated copper temperature distribution that will not be constant and will very likely not be $500^{\circ} \mathrm{F}$. Because removing the constant copper temperature assumption would add a number of complexities to this model (i.e. variable heat flux), it is prudent to avoid doing so. Instead, a more suitable constant copper temperature (other than $500^{\circ} \mathrm{F}$ ) is proposed given by the average of the copper temperature determined by equation (3.17) above. This value is then inserted into the equation below to yield a new heat transfer into the system.

$$
q_{\text {new }}=h_{\text {hot }} A_{\text {hot }}\left(T_{g h}-T_{\text {Cu,ave }}\right)
$$

After finding the updated heat transfer rate (termed " $q_{\text {new }}$ "), the steps preceding this point are recalculated. Doing so yields a "new" copper temperature distribution which requires that a second new heat transfer rate be calculated using equation (3.13) above and so on. 
This process is continued iteratively until the heat transfer is constant to five significant figures between iterations.

\section{III.2.2.4 Pressure Distribution and Rayleigh Line}

As was the case for temperature, the static pressure at any location $z$ in the annulus is a function of the inlet pressure, inlet Mach number and Mach number at location $z$ only. This relationship is given as

$$
P_{z}=P_{i n} \frac{\left(1+\gamma{M_{i n}}^{2}\right)}{\left(1+\gamma{M_{z}}^{2}\right)}
$$

In the above expression, $P_{i n}$ is the independently set inlet pressure, and $M_{\text {in }}$ and $M_{z}$ are the Mach numbers at the inlet and location $z$ respectively and are known from previous analyses. The stagnation pressure at any location $z$ in the annulus is also a function solely of the inlet stagnation pressure and the Mach number at the two points. The inlet stagnation pressure is found using the following relationship

$$
P_{o, i n}=P_{i n}\left(1+\frac{\gamma-1}{2} M_{i n}^{2}\right)
$$

The stagnation pressure at any location $z$ can then be calculated using the following equation (2): 


$$
P_{o, z}=P_{o, i n} \frac{\left(1+\gamma M_{i n}{ }^{2}\right)}{\left(1+\gamma M_{z}^{2}\right)}\left(\frac{1+\frac{\gamma-1}{2} M_{z}^{2}}{1+\frac{\gamma-1}{2} M_{i n}^{2}}\right)^{\frac{\gamma}{\gamma-1}}
$$

As was mentioned previously, a common reference for Rayleigh flow is the Rayleigh line which plots pressure on the ordinate versus specific volume on the abscissa. The Rayleigh line equation developed in Chapter II appears below:

$$
P_{1}-P_{2}=G^{2}\left(v_{2}-v_{1}\right)
$$

Because the pressure and temperature at the inlet are known, the density at the inlet is also known by the ideal gas law. The mass flux, $G$ is constant and known from continuity. Then, from the Rayleigh equation, if the pressure at any other point in the annulus is known, so is the density. Using this process, the Rayleigh line can be developed. While this approach is valid, a different one was used in this case. Since the static pressure and temperature distributions through the annulus have been solved for previously, the ideal gas law can be used in conjunction with these to find the static density distribution. In equation form, it amounts to:

$$
\rho=\frac{P}{R T}
$$

where $\mathrm{R}$ is the specific gas constant for Helium. The specific volume is then simply the inverse of density. 


\section{III.2.2.5 Thermal Choking}

It is important to predict whether or not the flow will reach the sonic point prior to the outlet of the annulus. If the flow were to choke before exiting the annulus, the modeling and analysis developed up to this point would be inaccurate. The velocity of the gas at each location $z$ can be calculated using the following equation derived from conservation of energy (2):

$$
u_{z}=\sqrt{u_{\text {in }}+2 C_{P}\left(T_{i n}-T_{z}\right)+2 Q_{i n-z}}
$$

The speed of sound at each location $z$ can be found using:

$$
c_{z}=\sqrt{\gamma R T_{z}}
$$

The ratio of these two values can then be taken to find the Mach number at each location $z:$

$$
M_{z}=\frac{u_{z}}{c_{z}}
$$

If the flow is found to reach a Mach number of unity before the outlet of the annulus, the inlet pressure should be increased to compensate until thermal choking is no longer a problem. 


\section{III.2.3 Helium Modeling Results}

The above model was run with the following inputs: (1) O/F of 2.0, (2) chamber pressure of $70 \mathrm{psia}$, (3) mass flow rate of $0.08 \mathrm{lbm} / \mathrm{sec}$, (4) inlet annulus temperature of $70^{\circ} \mathrm{F}$, and (5) inlet annulus pressure of 500 psia.

The predicted wall and gas temperatures versus distance through the annulus are shown in Figure 38 and Figure 39.

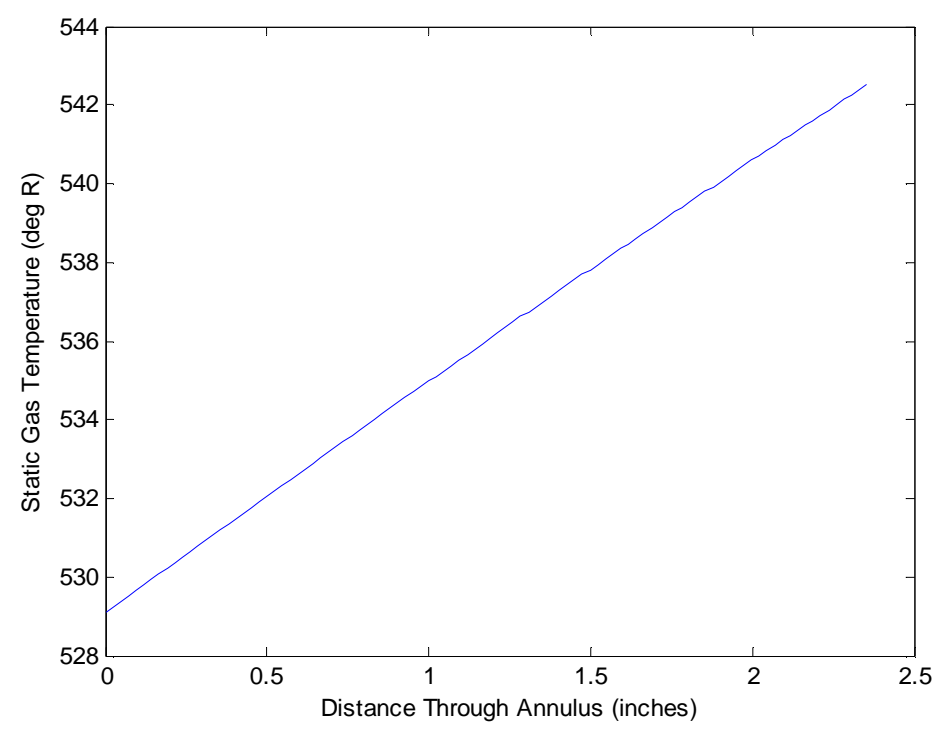

Figure 38. Predicted ideal gas static temperature 


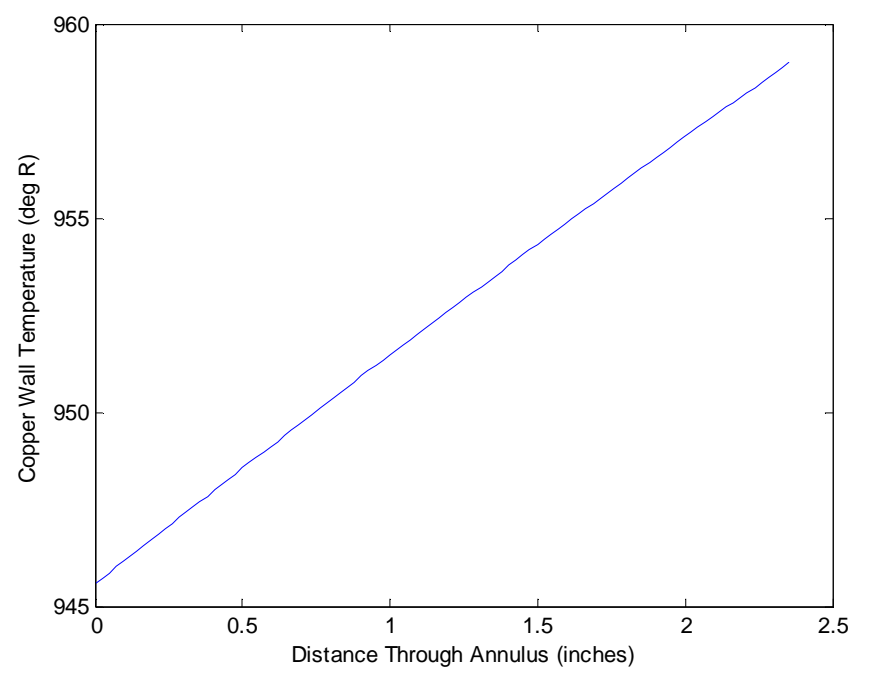

Figure 39. Predicted ideal gas copper wall temperature

The static and stagnation helium pressure plots are shown in Figure 40 and Figure 41 below. Notice that a much larger drop in static pressure than stagnation pressure is seen in this case. This is expected because the acceleration of the gas as it flows through the annulus recovers some of the stagnation pressure.

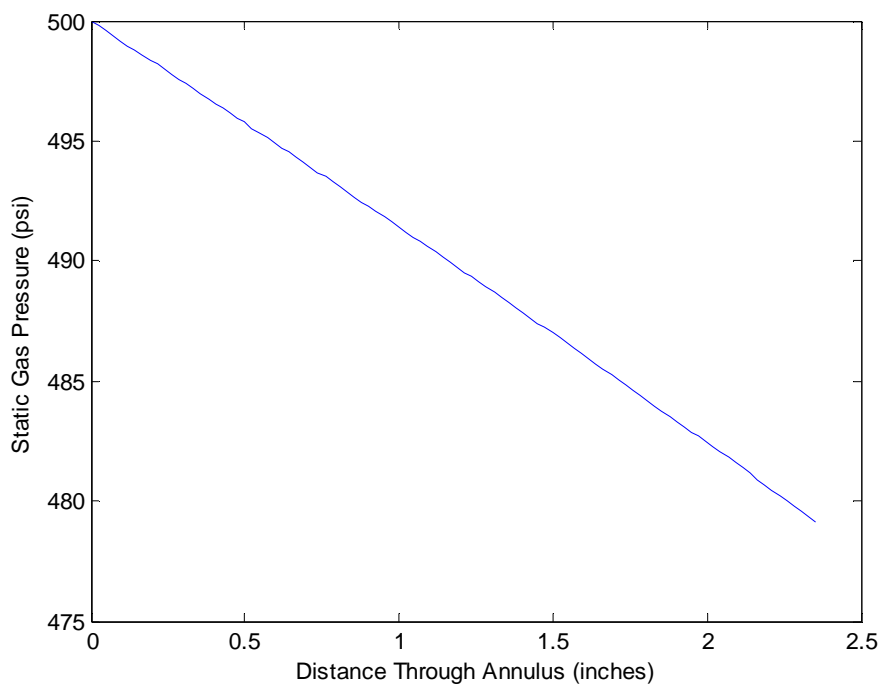

Figure 40. Predicted ideal gas static pressure 


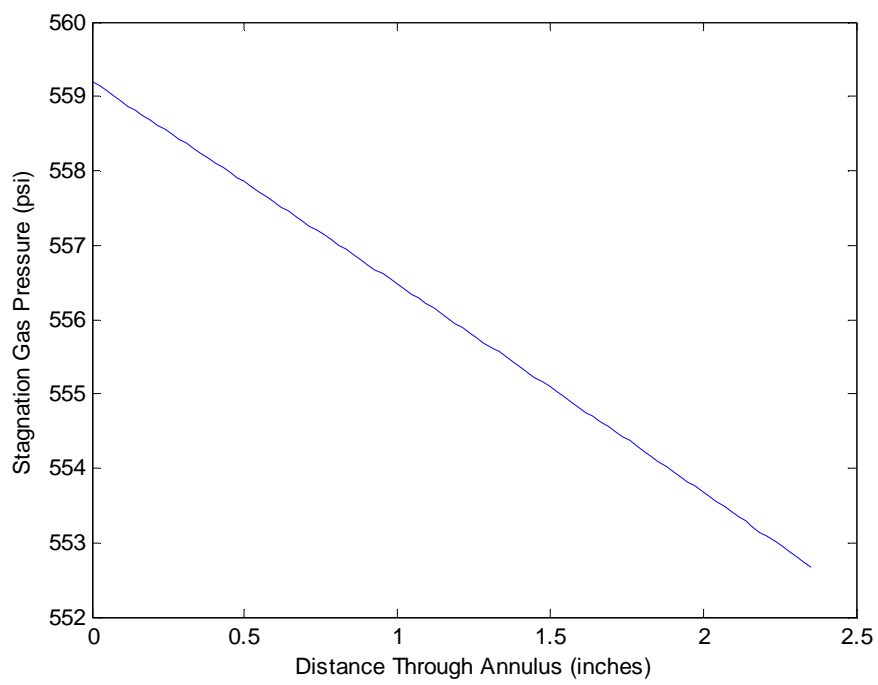

Figure 41. Predicted ideal gas stagnation pressure

The Rayleigh line for this flow is shown in Figure 42.

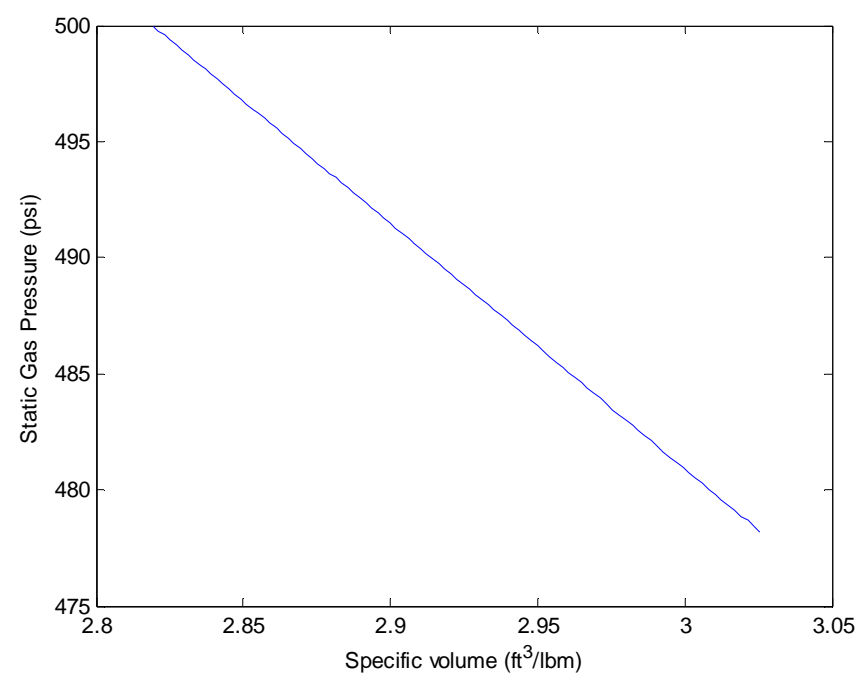

Figure 42. Predicted ideal gas Rayleigh line

It is also important to plot the Mach number as a function of distance through the annulus. The maximum Mach number should be sufficiently less than one to prevent 
thermal choking. Figure 43 shows the Mach number as a function of distance through the annulus.

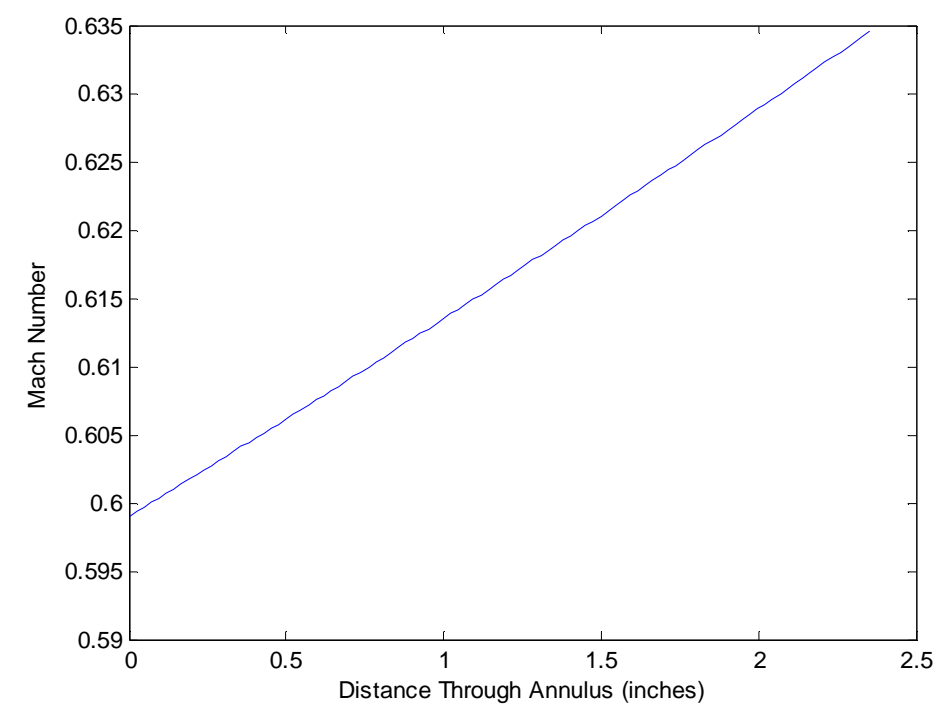

Figure 43. Predicted ideal gas Mach number

The results depicted above suggest that an ideal gas is capable of sufficiently cooling the system of interest for standard operating conditions.

\section{III.2.4 Finite Element Modeling}

In a separate thesis, a three dimensional finite element model (FEM) of the Rayleigh nozzle was created. The coolant heat transfer coefficient and static coolant temperatures derived analytically in sections III.2.1.2 and III.2.2.2 were input into the FEM. Similar, although not identical, hot side conditions were also input. It is important to note the differences between the hot side conditions input into the FEM and this analytical model. First, variable hot side heat transfer coefficients and combustion gas temperatures were 
used in the FEM. The analytical model assumes that the heat transfer coefficient and gas temperature derived at the throat are constant throughout the nozzle. In reality, these values will drop off quite dramatically longitudinally on either side of the throat. Accordingly, the FEM takes this into account by mapping variable hot side gas temperatures and heat transfer coefficients through the throat. Second, the analytical model uses an iterative approach that assumes the copper temperature is constant when calculating heat transfer rates whereas the FEM does not. Third, the analytical model presented does not account for the curvature in the coolant path. Fourth, the FEM accounts for conduction through the copper; the analytical model does not do this. Also along these lines, the FEM takes into consideration that the inlet and outlet of the coolant path are in close proximity to one another and are connected by a copper fin through which heat can conduct very easily given the large temperature gradient. The copper temperature contour plot produced by the FEM is shown in Figure 44.
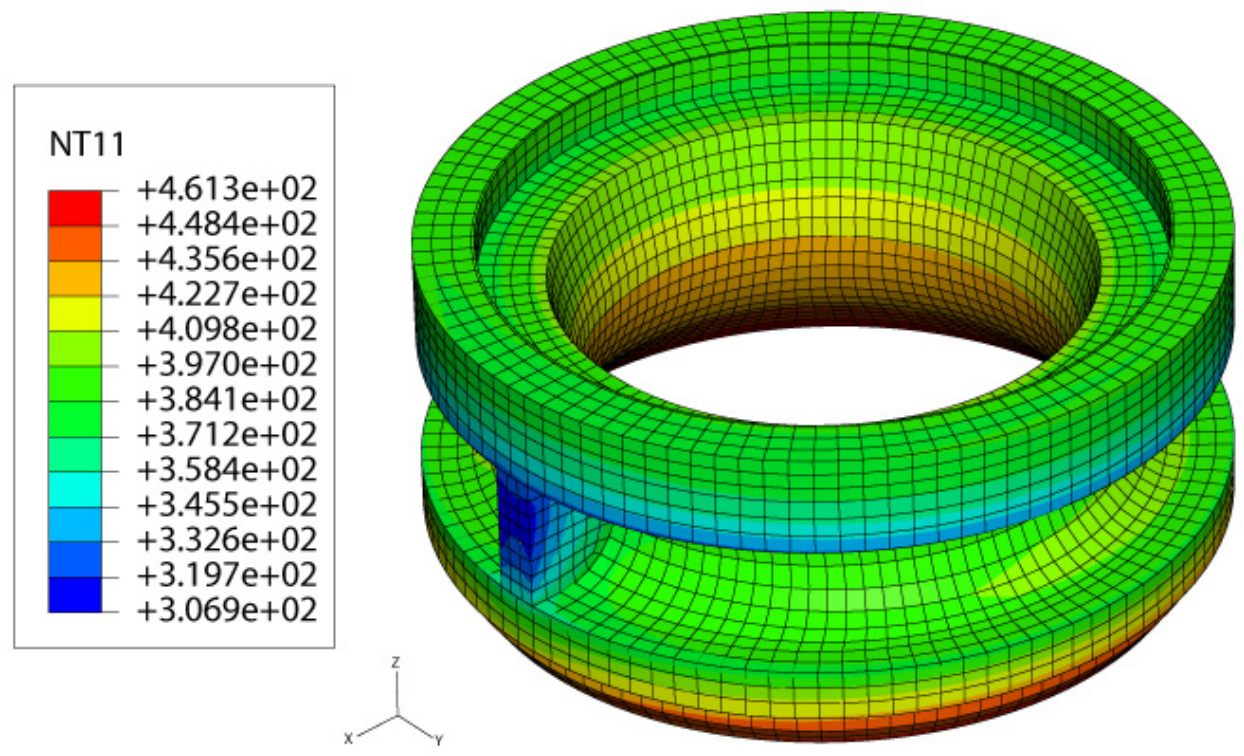

Figure 44. Temperature contour plot in degrees Fahrenheit of the copper insert 
The copper temperatures derived using both the analytical and finite element models are shown in Figure 45. Also shown in this plot are the helium gas temperatures that were common in both models.

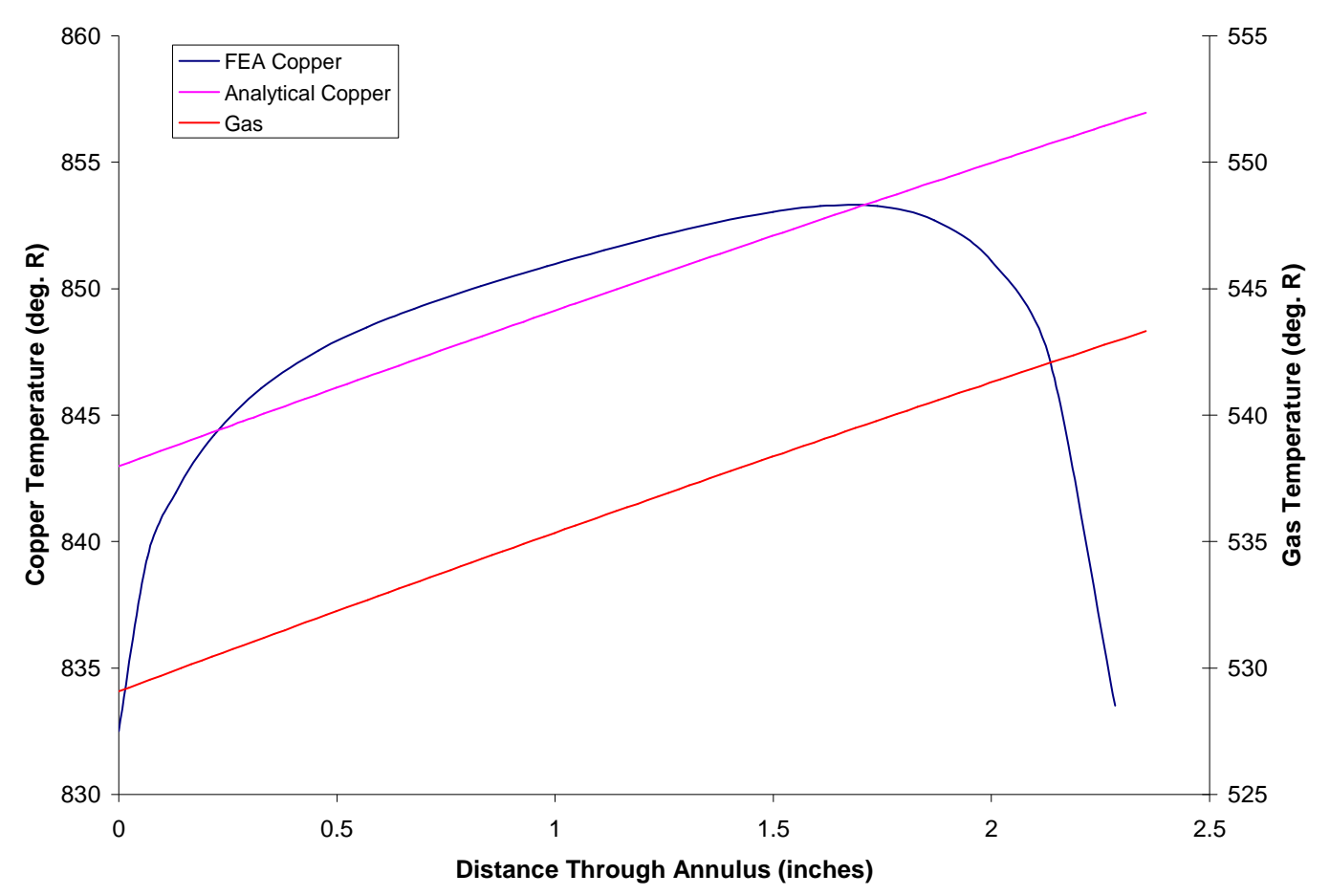

Figure 45. Comparing FE and analytical modeling predicted copper temperatures

First, it should be noted that the gas temperatures were derived analytically assuming an ambient inlet temperature and were used as an input for both the FEM and the analytical model. Next, notice that the gas temperature is parallel to the analytical copper temperature through the length of the annulus. This is a result of the assumption that the flow is thermally fully developed. By extension, anywhere that the FEM copper temperature line is parallel to the gas temperature line, the model is predicting thermally fully developed flow. Roughly, this occurs between points approximately $25 \%$ and $70 \%$ 
into the annulus. The slight variation in slopes in this region is likely due to the fact that the FEM model does not exactly model constant heat flux into the coolant. The drop in copper temperature at the beginning and end of the annulus is likely due to conduction through the copper from the coolant inlet side that is not accounted for in the analytical model.

The agreement between the analytical and finite element models results is promising. At worst, the two are within $25^{\circ} \mathrm{F}$ of each other and in most cases they match within $5^{\circ} \mathrm{F}$. It is important to note, however, that the validity of both of these models depends on the cold side heat transfer coefficient derived using the Petukhov Nusselt number correlation and the coolant gas temperatures derived using common gas dynamics analysis and assuming an inlet helium temperature of $70^{\circ} \mathrm{F}$. An error in either of these will skew the results of both the analytical and finite element models.

The analytical model developed in the previous section has confirmed that by lowering the combustion chamber pressure a perfect gas is capable of cooling the nozzle such that the maximum copper temperature does not exceed $500^{\circ} \mathrm{F}$. This result is confirmed by the finite element model. Sources of error include: (1) the assumption of thermally fully developed flow, (2) error in the empirical Nusselt number correlation used to derive the cold side heat transfer coefficient, (3) the constant copper temperature assumption, (4) neglecting shear and body forces, and (5) neglecting effects of the curvature and shape in the annulus by modeling it as a straight, round tube. The copper temperatures predicted 
by both the FEM and the analytical model suggest that it is reasonable to run an ideal gas experiment without worry of compromising the integrity of the copper insert.

\section{III.2.5 Ideal Gas Experiment Setup}

Note that the above analyses hinge on the ability to produce a helium mass flow rate of $0.08 \mathrm{lbm} / \mathrm{sec}$ with an annulus pressure of roughly $500 \mathrm{psia}$ (or high enough to avoid choking). In an attempt to replicate these conditions in the physical system, several modifications were necessary. These include: (1) calibration of a flow meter capable of monitoring helium flow rates, (2) removal of any restrictions in the system that may choke the flow, and (3) sizing of inlet and outlet injectors such that the mass flow rate is $0.08 \mathrm{lbm} / \mathrm{sec}$ and annulus pressure is roughly $500 \mathrm{psia}$. All three tasks were first performed with air to avoid wasting helium. A schematic of the ideal gas system including only the relevant equipment and information is given for reference in Figure 46.

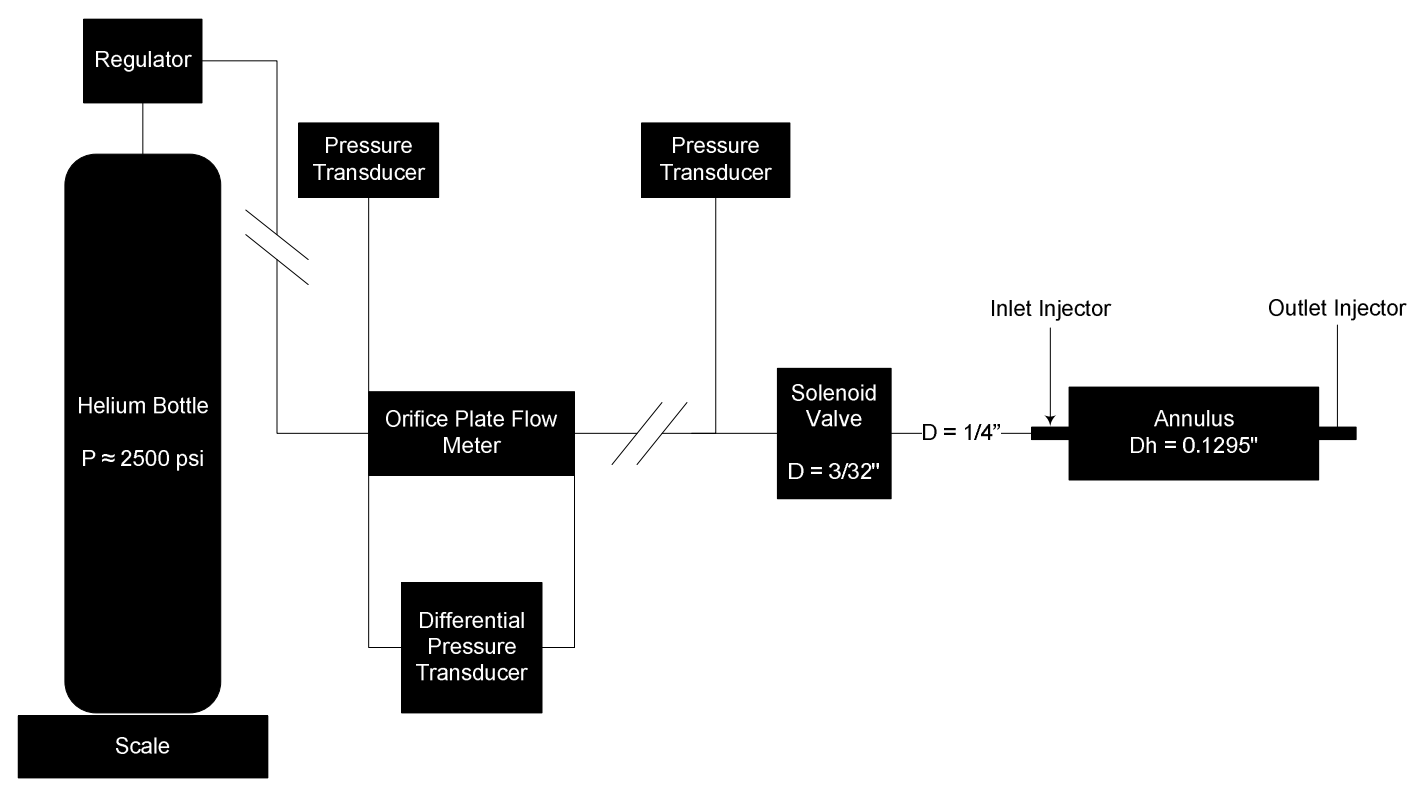

Figure 46. Simplified helium coolant system schematic 


\section{III.2.5.1 Helium Flow Meter Calibration}

An orifice plate flow meter was implemented to monitor the flow rate of helium through the system. A schematic of the flow meter is shown in Figure 47. A pressure transducer was connected to the pressure tap upstream of the orifice plate for monitoring pressure magnitude while a differential pressure transducer was connected to the pressure taps across the orifice plate to monitor pressure drop. The reported range of the differential pressure transducer was 0 and 200 inches of water $(0-7.6$ psi) which was initially suspected to be sufficient to measure the pressure drop for the expected flow rates. This instrumentation approach was taken rather than using a separate pressure transducer on each port as the scale of the differential pressure transducer provides superior accuracy to two individual pressure transducers.

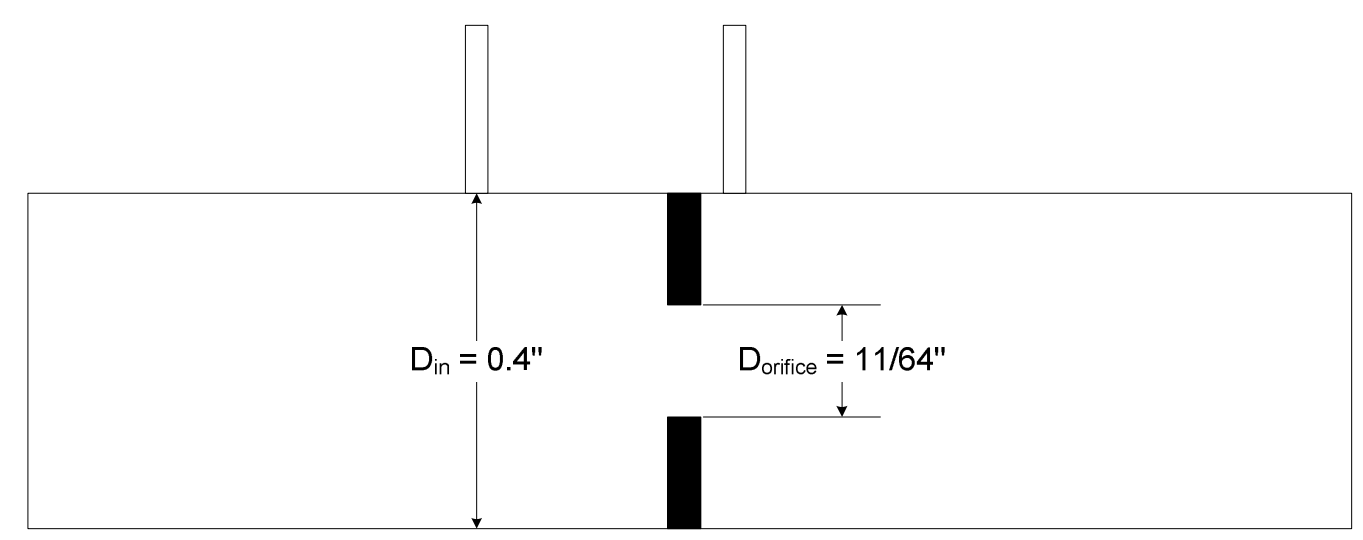

Figure 47. Orifice plate flow meter

Assuming the flow is incompressible, the general equation governing the flow rate through an orifice plate is given by Bernoulli's equation which simplifies to 


$$
\dot{m}=\frac{C_{d}}{\sqrt{1-\beta^{4}}} A_{\text {orifice }} \sqrt{2 \rho \Delta P}
$$

where $\beta$ is the ratio of the orifice to inlet diameter:

$$
\beta=\frac{D_{\text {orifice }}}{D_{\text {in }}}
$$

and $A_{\text {orifice }}$ is the cross-sectional area of the orifice. $\Delta P$ is the pressure drop across the orifice plate, $\rho$ is the density, and $C_{d}$ is the discharge coefficient. Because the flow is assumed incompressible, the density remains constant and is given by the ideal gas law in conjunction with the upstream conditions:

$$
\rho=\frac{P_{\text {in }}}{R T_{\text {in }}}
$$

The inlet temperature was assumed to be atmospheric at $70^{\circ} \mathrm{F}$. Empirically derived tables and charts exist to predict the discharge coefficient $C_{d}$ based on Reynolds number and $\beta$. In this case, a value of 0.6 was given as the estimated discharge coefficient. It was desirable to do some preliminary tests to confirm this value. These tests were first performed with air.

As air is a much heavier molecule than helium, the mass flow rate of air is expected to be much larger than that of helium for any given pair of injectors. An annulus inlet injector size was chosen such that the flow would choke at a flow rate low enough that the 
pressure drop across the orifice plate would not saturate the differential pressure transducer $\left(D_{\text {injector }}=0.04\right.$ inches $)$. As the test was running, the scale shown in Figure 46 monitored the weight of the air bottle as a function of time. After the test was complete, a linear trendline was fit to a weight versus time plot of the scale output giving a flow rate of $0.0159 \mathrm{lbm} / \mathrm{sec}$ with an R-squared value above 0.9 . It should also be noted that the trendline was very linear indicating a constant mass flow rate. The pressure transducer upstream of the orifice plate gave a reading of 699.3 psig while the differential pressure transducer read 18.8 inches of water. Solving equation (3.27) for the discharge coefficient gives

$$
C_{d}=\frac{\dot{m} \sqrt{1-\beta^{4}}}{A_{\text {orifice }} \sqrt{2 \rho \Delta P}}
$$

Substituting the measured parameters into equation (3.30) above gives a discharge coefficient of 0.641 . Note that it would be beneficial to conduct additional tests over a range of flow rates in order to obtain the discharge coefficient as a function of Reynolds number. This may be done at a later date but for the time being a discharge coefficient of 0.641 will be used in subsequent analyses. Note that this calibration was performed at a Reynolds number of 3.7E6.

\section{III.2.5.2 Checking the System for Choke Points}

It was necessary to determine whether or not any restrictions existed in the coolant system upstream of the annulus that may cause the ideal gas to choke at a flow rate lower 
than that desired. After an extensive review of the system, the point of minimum cross sectional area was determined to be a solenoid valve with a diameter of $3 / 32$ " just upstream of the annulus. The choked flow rate through this restriction is given by

$$
\dot{m}^{*}=\rho^{*} A^{*} c
$$

where $c$ is the speed of sound and is given by

$$
c=\sqrt{\gamma R T}
$$

and $\rho^{*}$ is the density of the fluid in the restriction which is a function of the pressure in the restriction as given by the ideal gas law. Since the pressure in the restriction is not known and cannot be easily derived due to the complicated geometry of the solenoid valve, a series of tests were performed to determine the choked mass flow rate of air through the solenoid restriction. Because the density of helium is significantly lower than that of air, its choked mass flow rate for a given orifice diameter is also lower. Based on this logic, if the maximum mass flow rate of air through the solenoid is less than the 0.08 $\mathrm{lbm} / \mathrm{sec}$ needed for the helium tests, it is necessary to remove the restriction from the system.

To perform this test, all components downstream of the solenoid valve were removed from the system. The valve was opened allowing the air to flow directly from the bottle through to solenoid to ambient pressure. The pressures just upstream of the valve were 
maintained at levels sufficiently high enough to ensure that the flow was choked (i.e. $\left.0.528 P_{\text {upstream }}>P_{\text {ambient }}\right)$. As the density in the solenoid restriction is dependent on the pressure just upstream of it, this test was run two times with variable regulator pressures. Note that the flow rate was too high to use the orifice plate flow meter so only the scale was available. However, this was deemed acceptable as the two flow rate monitoring methods agreed in previous tests. The results are shown in Table 4.

Table 4. Solenoid choking experimental and predicted mass flow rates

\begin{tabular}{|c|c|c|c|c|c|}
\hline & \multicolumn{2}{|c}{$\begin{array}{c}\text { Air Predicted Mass Flow Rate } \\
(\mathrm{lbm} / \mathrm{sec})\end{array}$} & $\begin{array}{c}\text { Helium Predicted Mass Flow } \\
\text { Rate }(\mathrm{lbm} / \mathrm{sec})\end{array}$ \\
\hline $\begin{array}{c}\text { Upstream } \\
\text { Pressure } \\
(\mathrm{psig})\end{array}$ & $\begin{array}{c}\text { Air Measured } \\
\text { Mass Flow } \\
\text { Rate } \\
(\mathrm{lbm} / \mathrm{sec})\end{array}$ & $\rho^{*}=\rho\left(P_{\text {upstream }}\right)$ & $\rho^{*}=\rho\left(P_{\text {ambient }}\right)$ & $\rho^{*}=\rho\left(P_{\text {upstream }}\right)$ & $\rho^{*}=\rho\left(P_{\text {ambient }}\right)$ \\
\hline 613 & 0.0519 & 0.169 & 0.004 & 0.068 & 0.002 \\
\hline 865 & 0.0724 & 0.239 & 0.004 & 0.098 & 0.002 \\
\hline
\end{tabular}

Based on these results, it was decided to remove the solenoid valve from the system. This posed no major problems other than the need to open and close a separate manual valve to run the coolant system in subsequent tests.

\section{III.2.5.3 Injector Sizing}

Based on the results of the solenoid choke test and previous preliminary tests, several experiments were run with both air and helium in order to attempt to choose an inlet and outlet injector size that would give a mass flow rate of $0.08 \mathrm{lbm} / \mathrm{sec}$ with an annulus pressure around 500 psi (or sufficiently high enough to avoid thermally choking the flow). These tests were first run with air to avoid wasting helium. It was noted based on 
previous tests and analyses that for a given injector size, the mass flow rate of air must be significantly higher (approximately two times) that desired with helium. The largest available inlet and outlet injectors both with diameters of 0.125 " were used for this test. The results of the first two tests (both using air) are given in Table 5.

Table 5. Air injector test results $(0.125 "-0.125 ")$

\begin{tabular}{|c|c|c|}
\hline $\begin{array}{c}\text { Upstream } \\
\text { Pressure } \\
\text { (psig) }\end{array}$ & $\begin{array}{c}\text { Annulus Inlet } \\
\text { Pressure } \\
\text { (psig) }\end{array}$ & $\begin{array}{c}\text { Mass Flow Rate } \\
\text { (lbm/sec) }\end{array}$ \\
\hline 567 & 401 & 0.0827 \\
\hline 641 & 453 & 0.0958 \\
\hline
\end{tabular}

Note that the pressure drop across the exit injector was sufficiently high to choke the flow. The flow rates observed are far lower than those necessary to sufficiently cool the system in a hot fire test (about $0.16 \mathrm{lbm} / \mathrm{sec}$ ). Based on these results, and those of the solenoid tests, it was concluded that the system should be tested with the largest possible injectors flowing helium. Based on the need to keep the injectors at least slightly smaller than the annulus ( $D_{h}=0.1295$ inches), the 0.125 " injectors were both reamed out to 0.128 ". A test was then performed with this configuration with helium as the working fluid. In a preliminary test, the flow meter differential pressure transducer saturated and was changed to one with a significantly larger operating range $(0-100 \mathrm{psi})$. The final $0.128 "-0.128$ " helium injector test gave the following results: 
Table 6. Helium injector test results (0.128" - 0.128")

\begin{tabular}{|c|c|c|c|c|c|c|}
\hline $\begin{array}{c}\text { Upstream } \\
\text { Pressure } \\
\text { (psig) }\end{array}$ & $\begin{array}{c}\text { Annulus } \\
\text { Inlet } \\
\text { Pressure } \\
\text { (psig) }\end{array}$ & $\begin{array}{c}\text { Annulus } \\
\text { Outlet } \\
\text { Pressure } \\
\text { (psig) }\end{array}$ & $\begin{array}{c}\text { Flow Meter } \\
\text { Pressure } \\
\text { Drop } \\
\text { (psi) }\end{array}$ & $\begin{array}{c}\text { Flow Meter } \\
\text { Downstream } \\
\text { Pressure } \\
\text { (psig) }\end{array}$ & $\begin{array}{c}\text { Flow Meter } \\
\text { Flow Rate } \\
\text { (lbm/sec) }\end{array}$ & $\begin{array}{c}\text { Scale Mass } \\
\text { Flow Rate } \\
\text { (lbm/sec) }\end{array}$ \\
\hline 704 & 482 & 429 & 36.8 & 664 & 0.0409 & 0.0367 \\
\hline
\end{tabular}

The crucial problem presented by these results is that the maximum possible flow rate through the system is only about half that necessary to maintain predicted copper temperatures below $500^{\circ} \mathrm{F}$ based on the conservative assumptions stated previously. The second issue given by these results is that the size of the injectors necessary to provide mass flow rates even on the order of what is desired result in severe frictional pressure drops within the annulus. Essentially, with these large injectors a Fanno model of the system becomes more relevant and necessary. The Rayleigh flow model presented in section III.2.2 was run with an inlet annulus pressure of 482 psia and a mass flow rate of $0.04 \mathrm{lbm} / \mathrm{sec}$. This gave an expected pressure drop from heat load alone of only $8 \mathrm{psi}$ which is less than one quarter of that seen from friction in the experimental results. The Rayleigh model results also predict a maximum copper wall temperature of $685^{\circ} \mathrm{F}$ which is higher than the allowable $500^{\circ} \mathrm{F}$.

Two conclusions result from the preliminary experiments presented above. First, it is necessary to reduce the heat load as much as possible to compensate for the loss in flow rate. This is accomplished by reducing the size and number of oxidizer injectors into the combustion chamber. Second, it is necessary to formulate a Fanno model of the system to 
predict the magnitude of frictional pressure drops. Both of these steps are taken prior to running the experiments.

\section{III.2.6 Fanno Flow Model}

Per the conclusions of the previous section, a Fanno model of the system is developed. Implementing this model was significantly less complex than the Rayleigh model. The process and governing equations are presented in this section.

The properties at the inlet of the annulus are input and derived identically to those of the Rayleigh model (see section III.2.2). Then, assuming friction is the only significant driving force, the Mach number at each location in the annulus is derived using the following equation from Zucrow and Hoffman (2):

$$
\frac{4 f z}{D_{h}}=\frac{1}{\gamma{M_{i n}}^{2}}\left(\frac{{M_{z}}^{2}-M_{i n}{ }^{2}}{{M_{z}}^{2}}\right)+\frac{\gamma+1}{2 \gamma} \ln \left(\frac{{M_{i n}}^{2}\left(1+\frac{\gamma-1}{2} M_{z}{ }^{2}\right)}{{M_{z}}^{2}\left(1+\frac{\gamma-1}{2} M_{i n}{ }^{2}\right)}\right)
$$

where $z$ is distance in the annulus from the point where the inlet conditions are defined and $M_{i n}$ is the Mach number at that location. Finally, $f$ is the friction factor and is given by the Colebrook empirical relation

$$
\frac{1}{\sqrt{f}}=-2 \log \left(\frac{e / D_{h}}{3.7}+\frac{2.51}{R e \sqrt{f}}\right)
$$


where $e$ is the surface roughness. In this case, the surface is assumed to be smooth, giving a value of zero for $e$. Because the Reynolds number is constant throughout the annulus, equation (3.34) need only be solved once, giving a single friction factor for the entire annulus. Equation (3.33) is then solved for the Mach number at each location $z$. Finally, the pressure at each location in the annulus is a function simply of the inlet pressure and the Mach numbers

$$
P_{1}-P_{z}=P_{\text {in }}\left(\frac{{M_{\text {in }}}^{2}}{{M_{z}^{2}}^{2}}\right)\left(\frac{1+\frac{\gamma-1}{2} M_{\text {in }}^{2}}{1+\frac{\gamma-1}{2} M_{z}^{2}}\right) P_{2}=G^{2}\left(v_{2}-v_{1}\right)
$$

Again, equation (3.35) is solved to give the pressure at each location $z$ in the annulus as predicted by the Fanno model in which the pressure drop is due to friction alone.

\section{III.2.7 Helium Experiments}

Two separate Helium cooled experiments were run. The first utilized only two oxidizer injectors into the combustion chamber to minimize chamber pressure and $\mathrm{O} / \mathrm{F}$, thus reducing the heat load. The second included four combustion chamber oxidizer injectors to increase heat load. The data post-processing and results are summarized below. It should be noted that in both of these tests, the electrical system maintaining the thermocouples was incorrectly grounded resulting in a phenomena called "ground looping." This caused inaccurate copper and gas temperature measurements. As this issue was not noticed until after the ideal gas experiments had been run, it was desirable to salvage the collected data. To do so, an attempt was made to quantify the effects of 
ground-looping. More specifically, a method of "converting" the inaccurately measured temperatures to physical temperatures. An experiment was setup in which the system was uniformly heated with a heat gun to $500^{\circ} \mathrm{F}$ after which it was allowed to cool. A previously calibrated and appropriately grounded thermocouple was attached to the copper. During the cooling process, the reference thermocouple and improperly grounded copper/gas thermocouples were read periodically. This method provided the correction functions given in Figure 48.

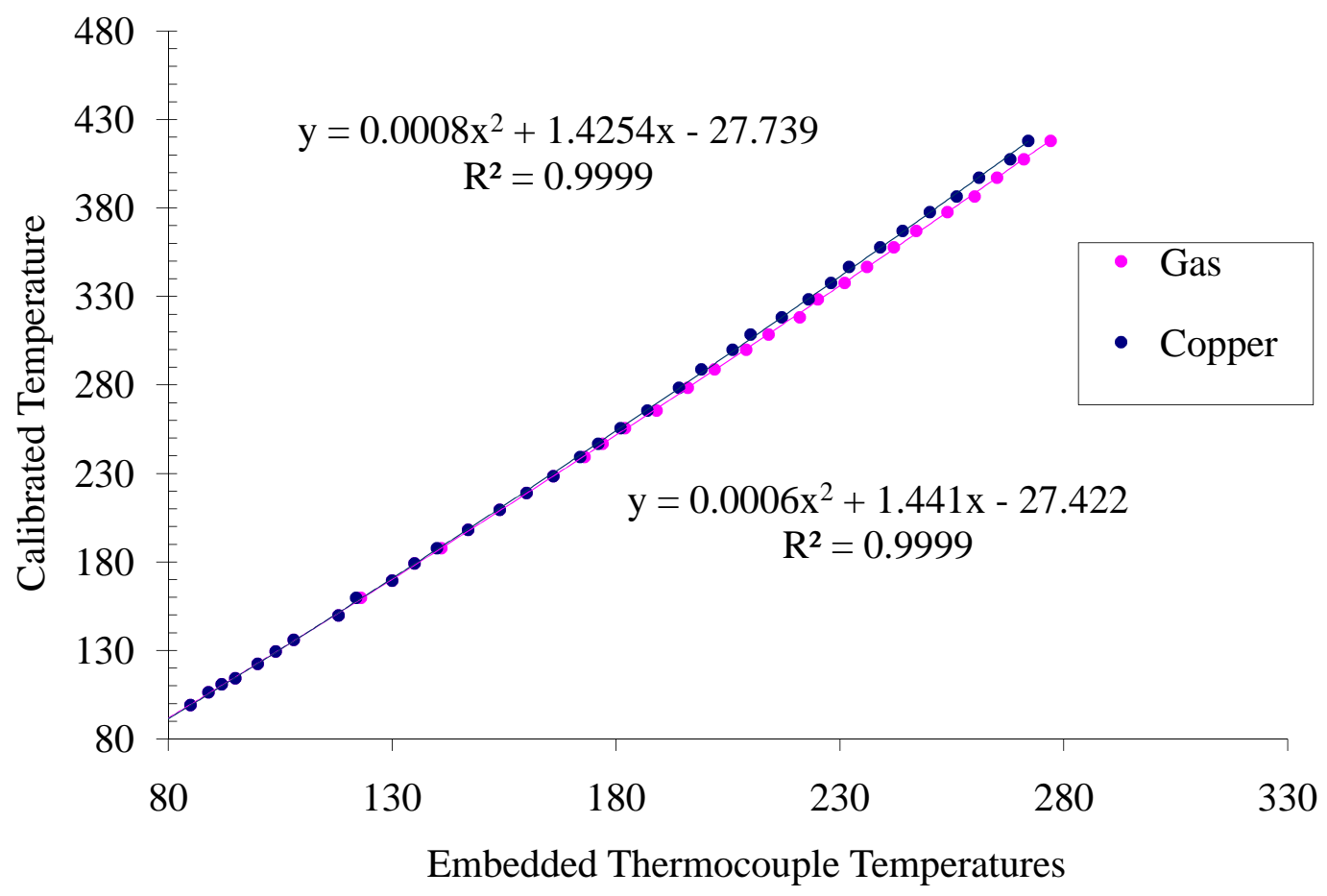

Figure 48. Correcting for ground-looping in gas and copper temperature measurements

It should be noted that all temperatures presented in the following sections are the measured temperatures corrected using these functions. 


\section{III.2.7.1 First Ideal Gas Cooled Experiment}

Two combustion chamber oxidizer injectors were used in this test, giving a chamber pressure of 50 psia and an $\mathrm{O} / \mathrm{F}$ of 2.15. Substituting these values into TEP and the Bartz equation gives a static combustion gas temperature and heat transfer coefficient at the throat of the nozzle of $2709^{\circ} \mathrm{F}$ and $1.04 \mathrm{E}-3 \mathrm{BTU} / \mathrm{in}^{2} \mathrm{sec}^{\circ} \mathrm{R}$ respectively. After evaluating the transient data, the region between 36 seconds and 42 seconds was judged to be relatively steady state. Transient plots of copper temperature, gas temperature and gas pressure in the steady state region are shown in Figure 49, Figure 50, and Figure 51.

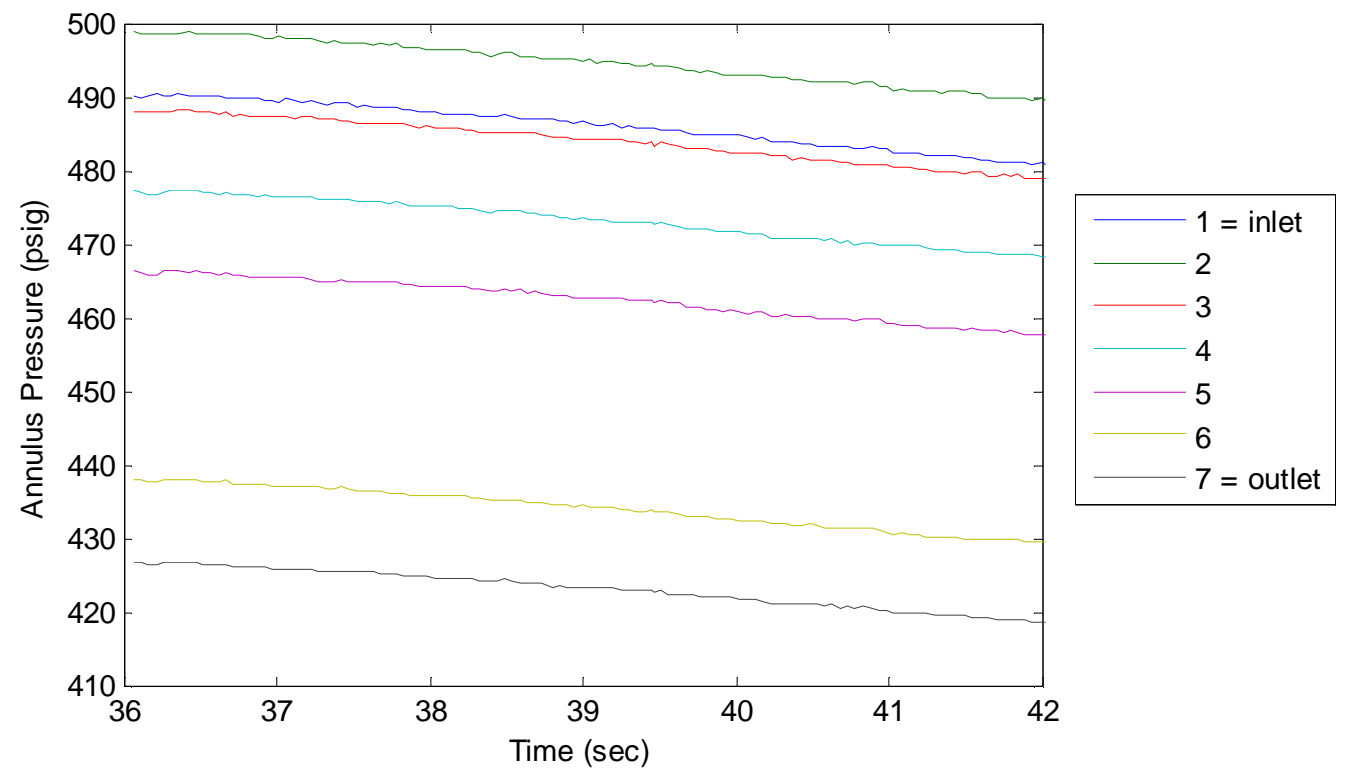

Figure 49. Helium test 1: transient pressure profiles in the steady state region (Run 66) 


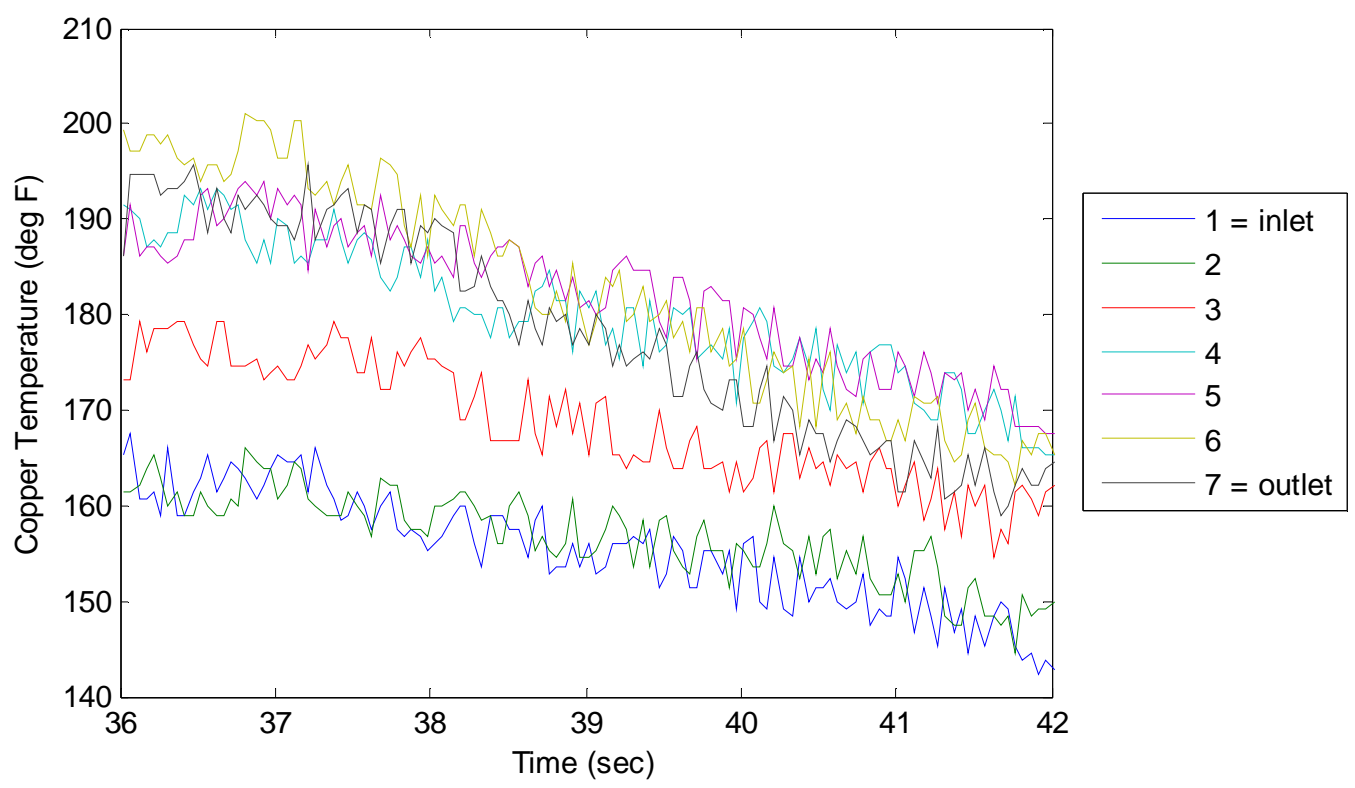

Figure 50. Helium test 1: transient copper temperature profiles in the steady state region (Run 66)

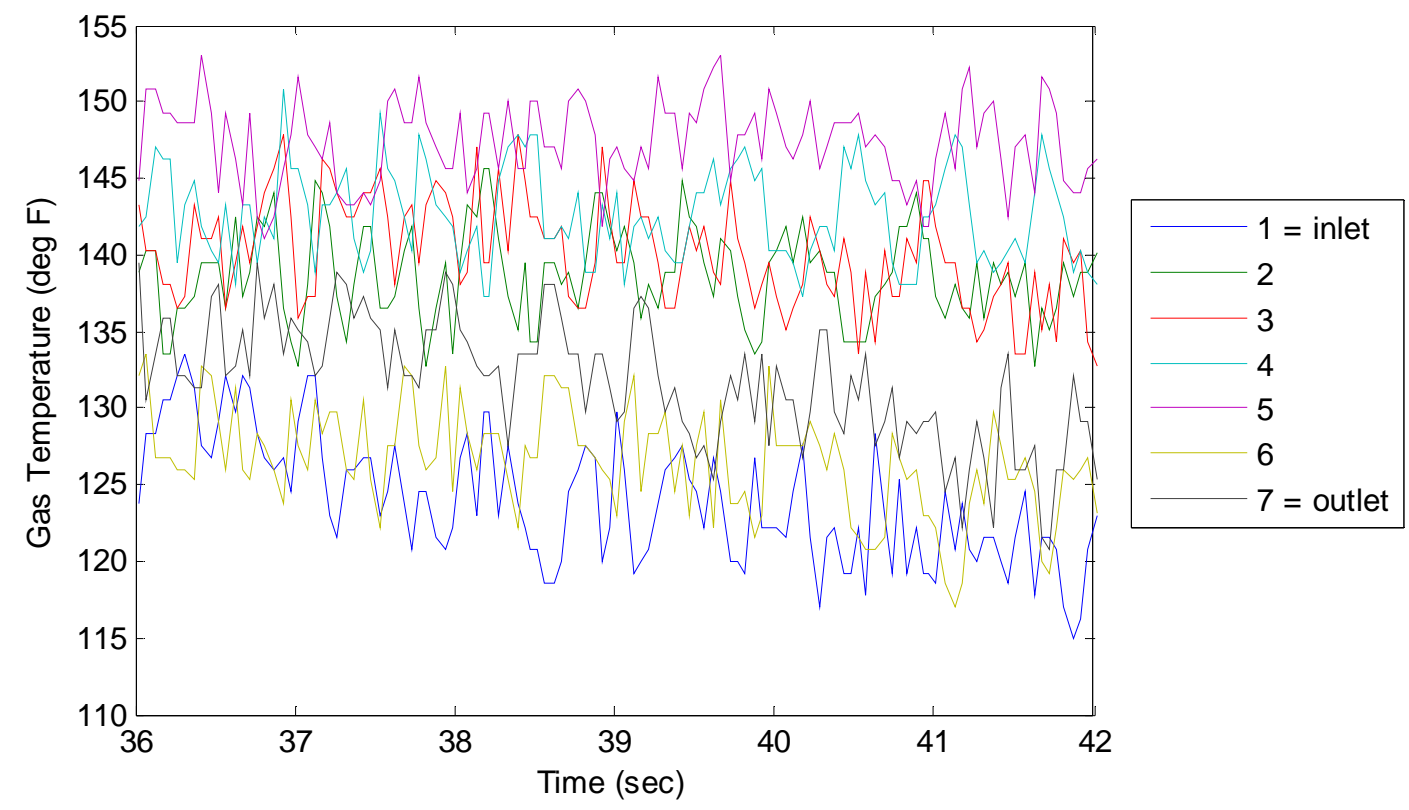

Figure 51. Helium test 1: transient gas temperature profiles in the steady state region (Run 66) 
Finally, the mass flow rate as measured by the orifice plate flow meter in the steady-state region is given in Figure 52.

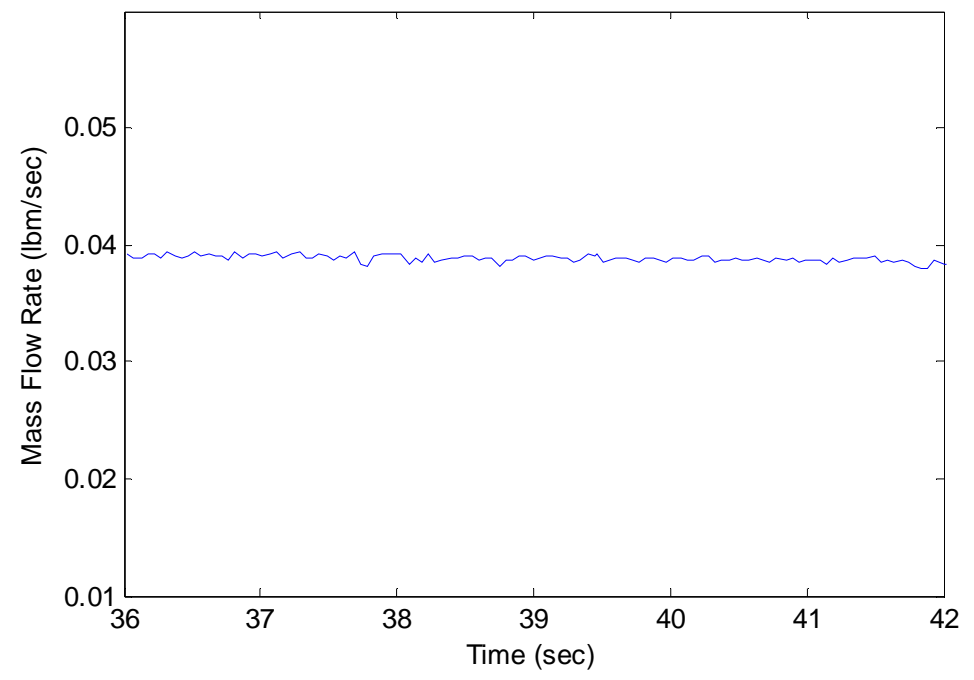

Figure 52. Helium test 1: transient mass flow rate profile in the steady state region (Run 66)

The temperatures, pressures and mass flow rate given in the above plots are averaged over the steady state region to give a single steady state value. Recall that the Rayleigh and Fanno analytical models require the temperature and pressure at the annulus inlet as well as mass flow rate as inputs. Accordingly, the first measured helium pressure and temperature are input in conjunction with the mass flow rate into the MATLAB routine given in Appendix A. Figure 53 compares the experimental and predicted gas pressures through the annulus. Note that the error bars shown on the experimental data points indicated the transducer resolution of \pm 2.5 psia. Also note that the Rayleigh and Fanno models predict pressure drop from heat addition and friction respectively. The prediction 
of both of these models individually as well as the sum of their individual contributions is shown in Figure 53.

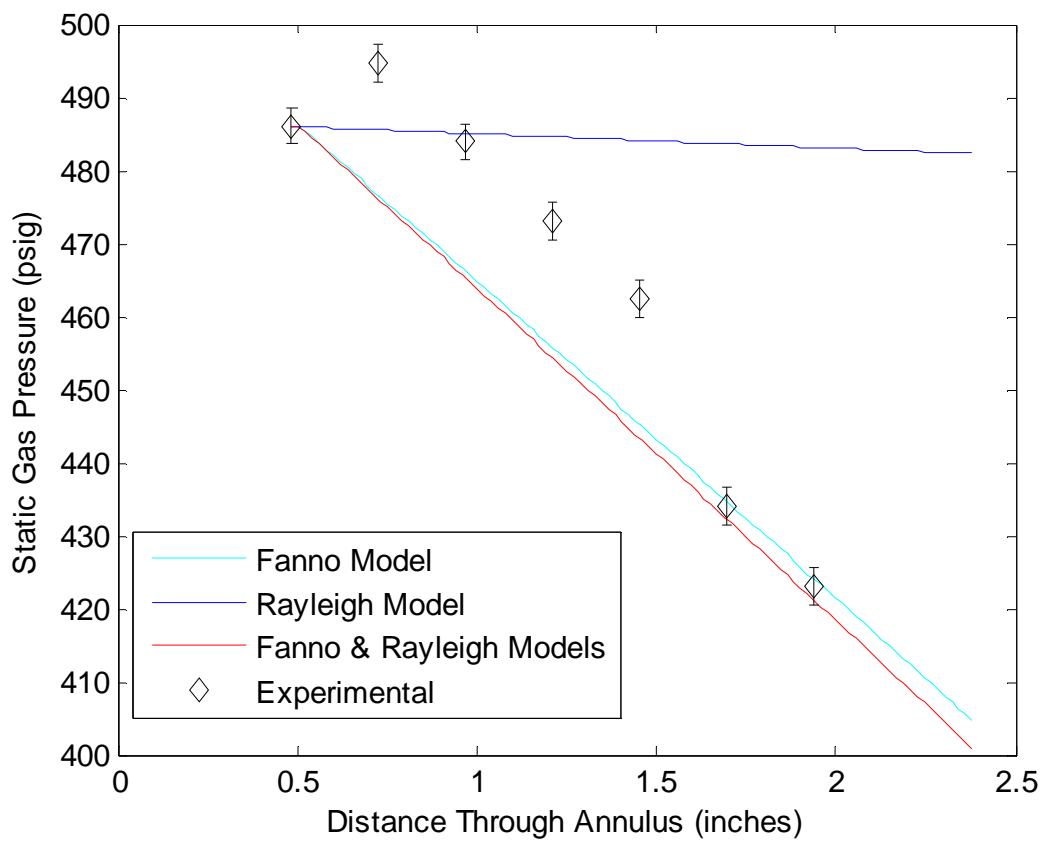

Figure 53. Helium test 1: predicted and measured average steady-state pressures

The static helium temperatures through the annulus are given in Figure 54. Note that the Fanno model was developed primarily to predict pressure drops due to friction, not temperature changes. Appropriately, only the Rayleigh contribution to temperature change is shown. 


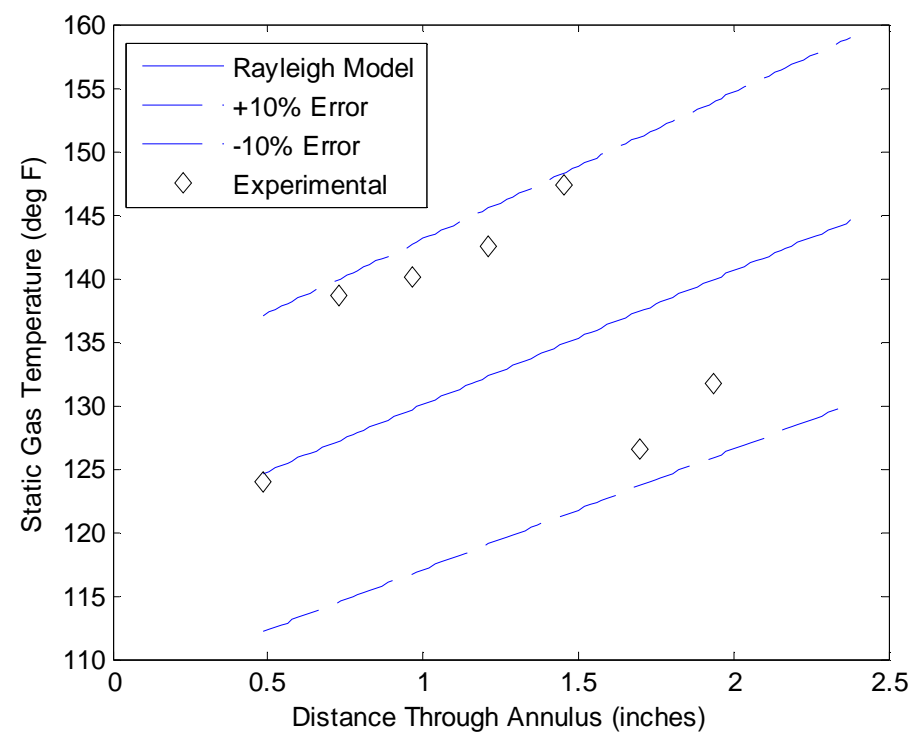

Figure 54. Helium test 2: predicted and measured average gas temperatures

Notice that similar to the pressure, there appears to be a decrease in temperatures at the beginning and end of the annulus. The predicted and measured copper temperatures are given in Figure 55..

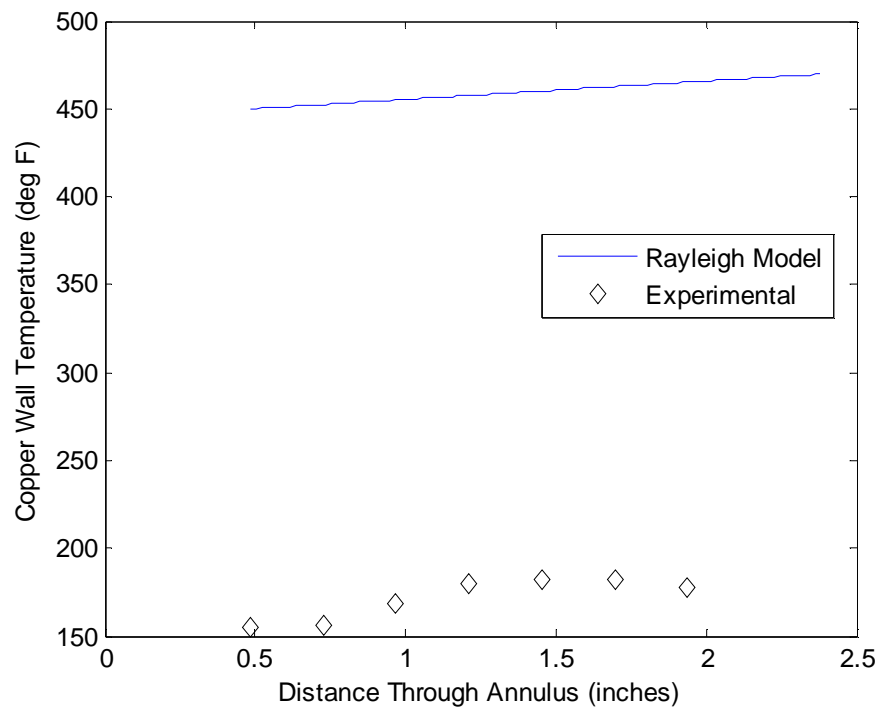

Figure 55. Helium test 1: predicted and measured steady state wall temperatures 
There is obviously a significant discrepancy between the magnitude of the measured and predicted copper temperatures. The source of this difference will be discussed in subsequent sections. However, to ascertain whether or not the trends are reasonable the copper temperature increase from the inlet value is given in Figure 56.

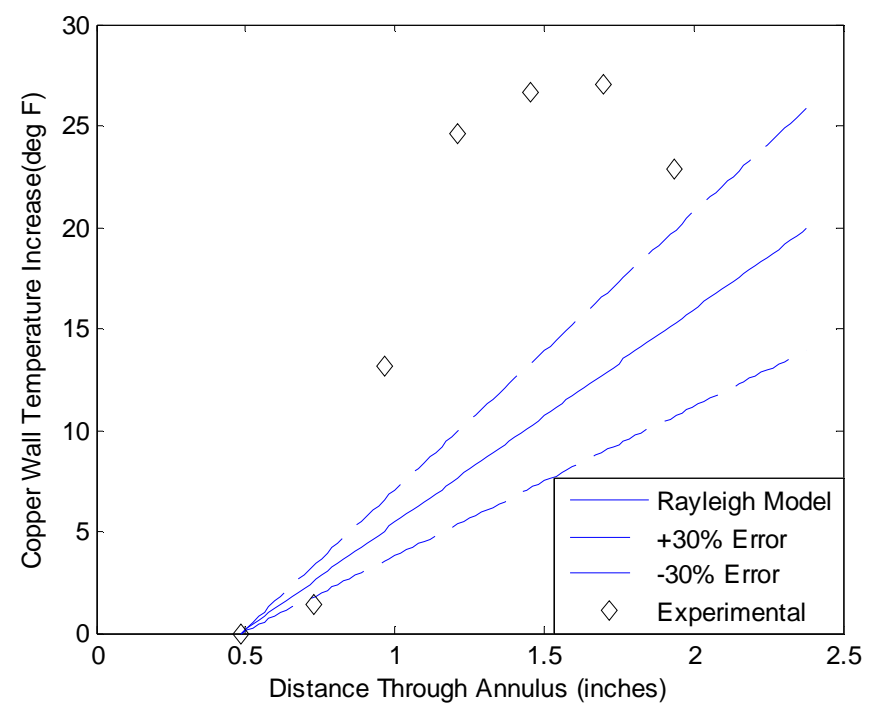

Figure 56. Helium test 1: predicted and measured copper wall temperature increase

Finally, the heat transfer coefficients predicted using the Dittus-Boelter correlation and those derived from experimental data are shown in Figure 57. 


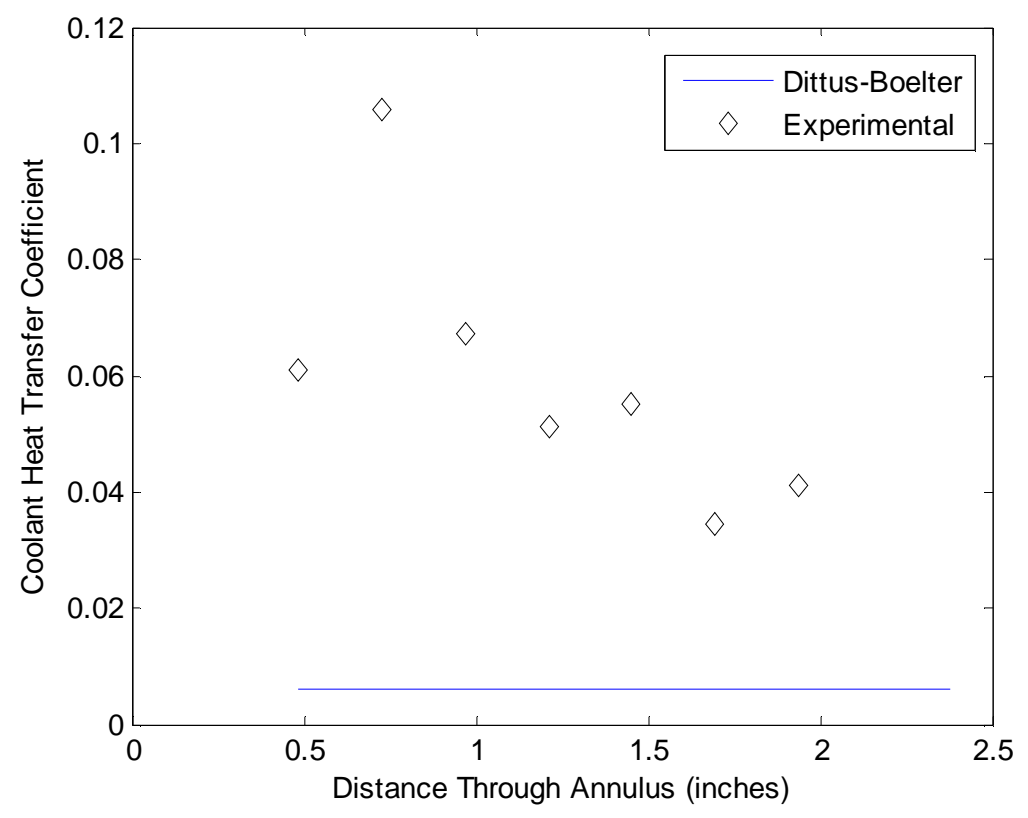

Figure 57. Helium test 1: predicted and experimental coolant heat transfer coefficients

Note that the unusually low magnitude of the measured copper temperatures is responsible for the large discrepancy between the predicted and measured heat transfer coefficients. Sources of this discrepancy are discussed in subsequent sections.

\section{III.2.7.2 Second Ideal Gas Cooled Experiment}

To test the repeatability of the ideal gas experiment, a second run was performed. To increase the heat load, four combustion chamber oxidizer injectors were implemented to yield a chamber pressure of $121 \mathrm{psia}$ and an $\mathrm{O} / \mathrm{F}$ of 1.9. These values were input into TEP and the Bartz equation to yield a static combustion gas temperature of $2851^{\circ} \mathrm{F}$ and heat transfer coefficient of $1.96 \mathrm{E}-3 \mathrm{BTU} / \mathrm{in}^{2} \mathrm{sec}^{\circ} \mathrm{R}$. Because this test was significantly longer than the previous one, a more extensive steady state region was available and chosen 
between 45 and 60 seconds. The transient plots of the characteristics of interest are given in the following four figures.

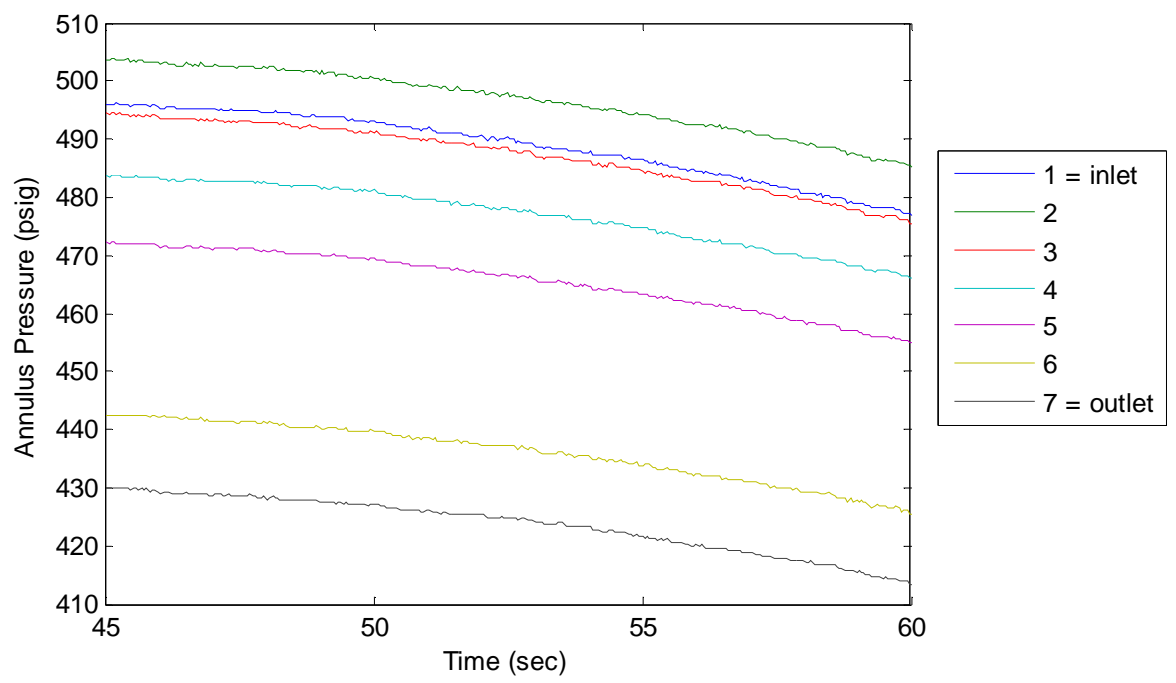

Figure 58. Helium test 2: transient pressure profiles in the steady state region (Run 67)

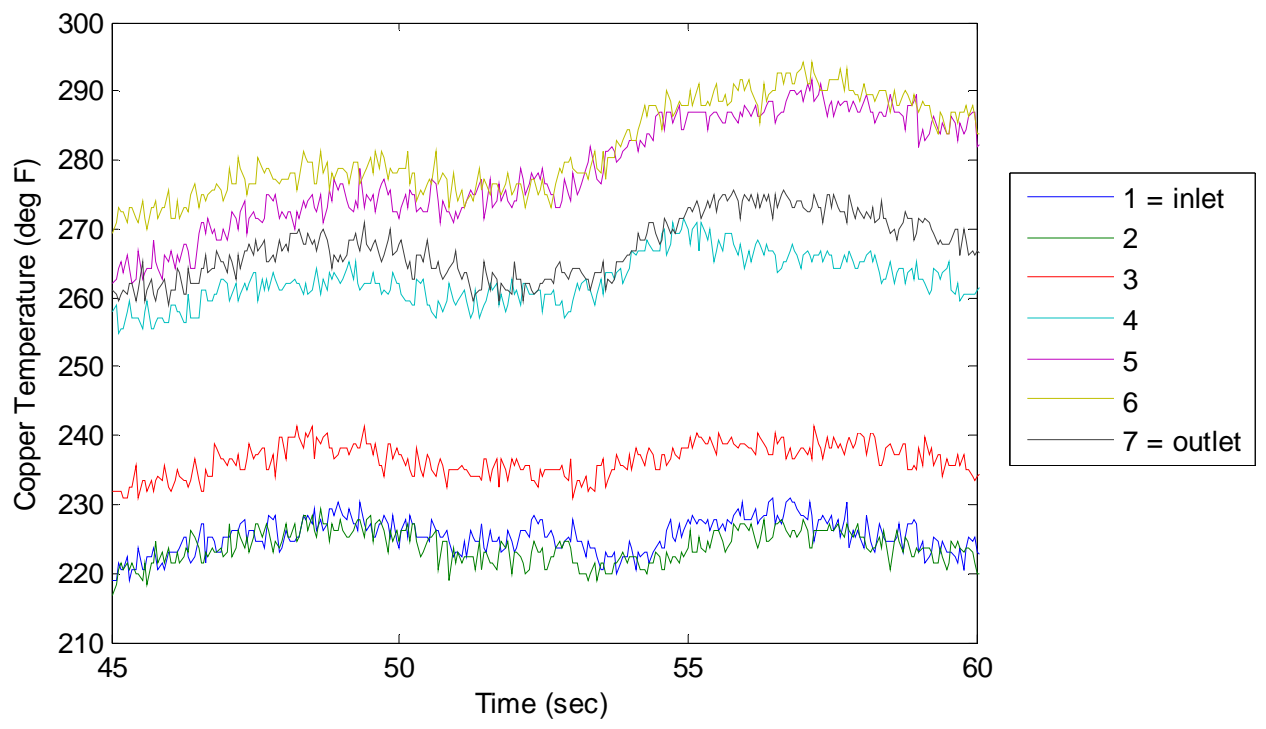

Figure 59. Helium test 2: transient copper temperature profiles in the steady state region (Run 67) 


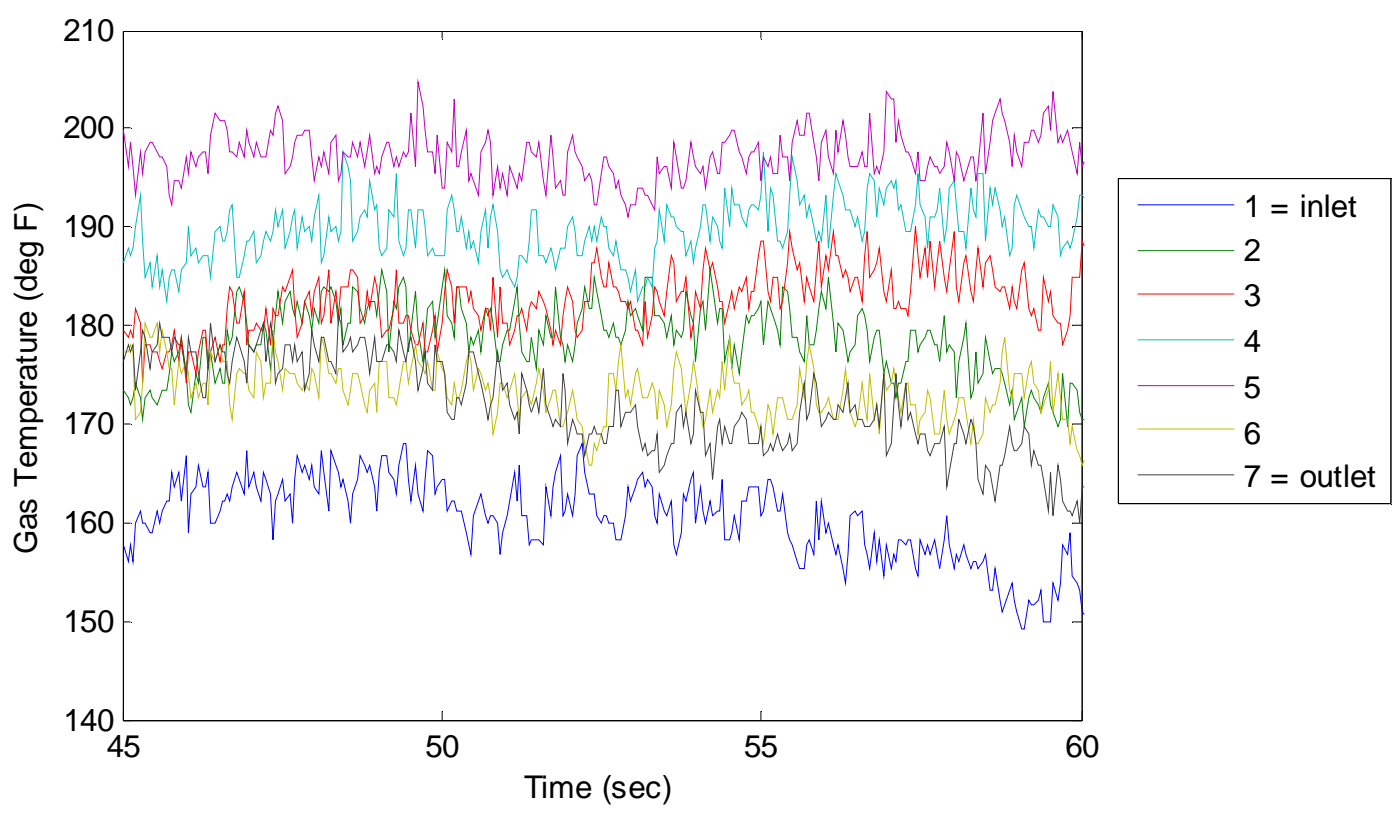

Figure 60. Helium test 2: transient gas temperature profiles in the steady state region (Run 67)

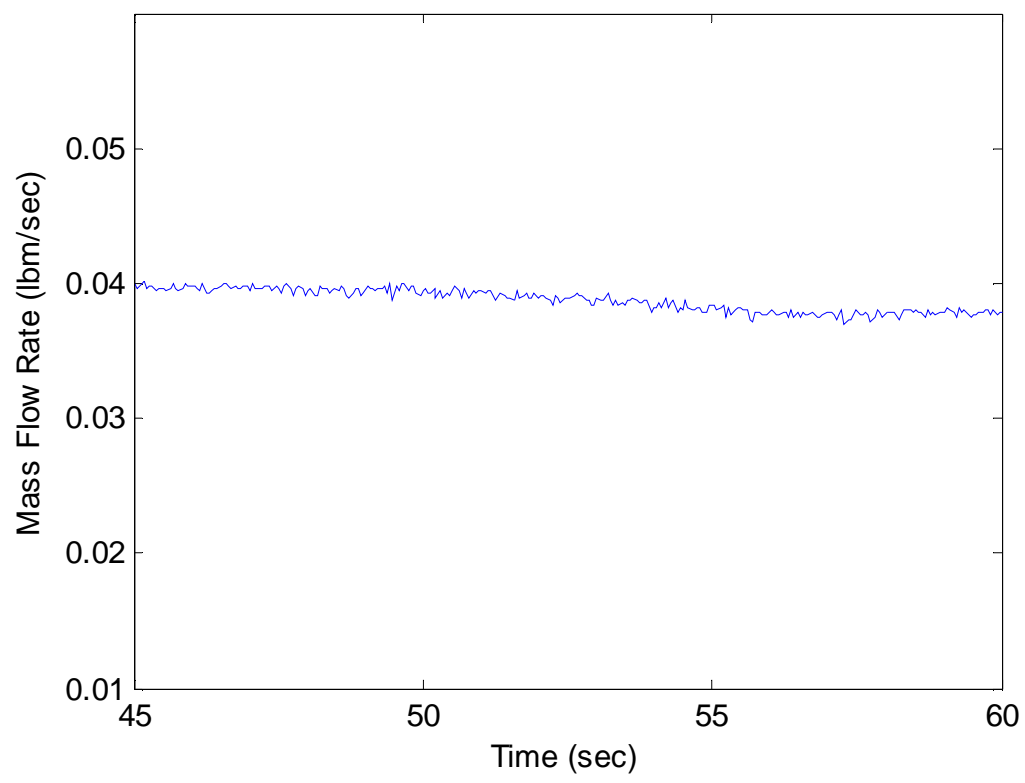

Figure 61 . Helium test 2: mass flow rate profile in the steady state region (Run 67) 
Note that any fluctuations in the above plots are due to unexpected changes on the hot side during the combustion process (most notably, fluctuations in chamber pressure). While these fluctuations are not ideal, because of the number of data points over which the steady-state values are averaged, they are tolerable. The combustion gas heat transfer coefficient and static temperature along with coolant inlet parameters were input into the Rayleigh and Fanno models. The results for this test are strikingly similar to those from the previous test and are given in the figures below.

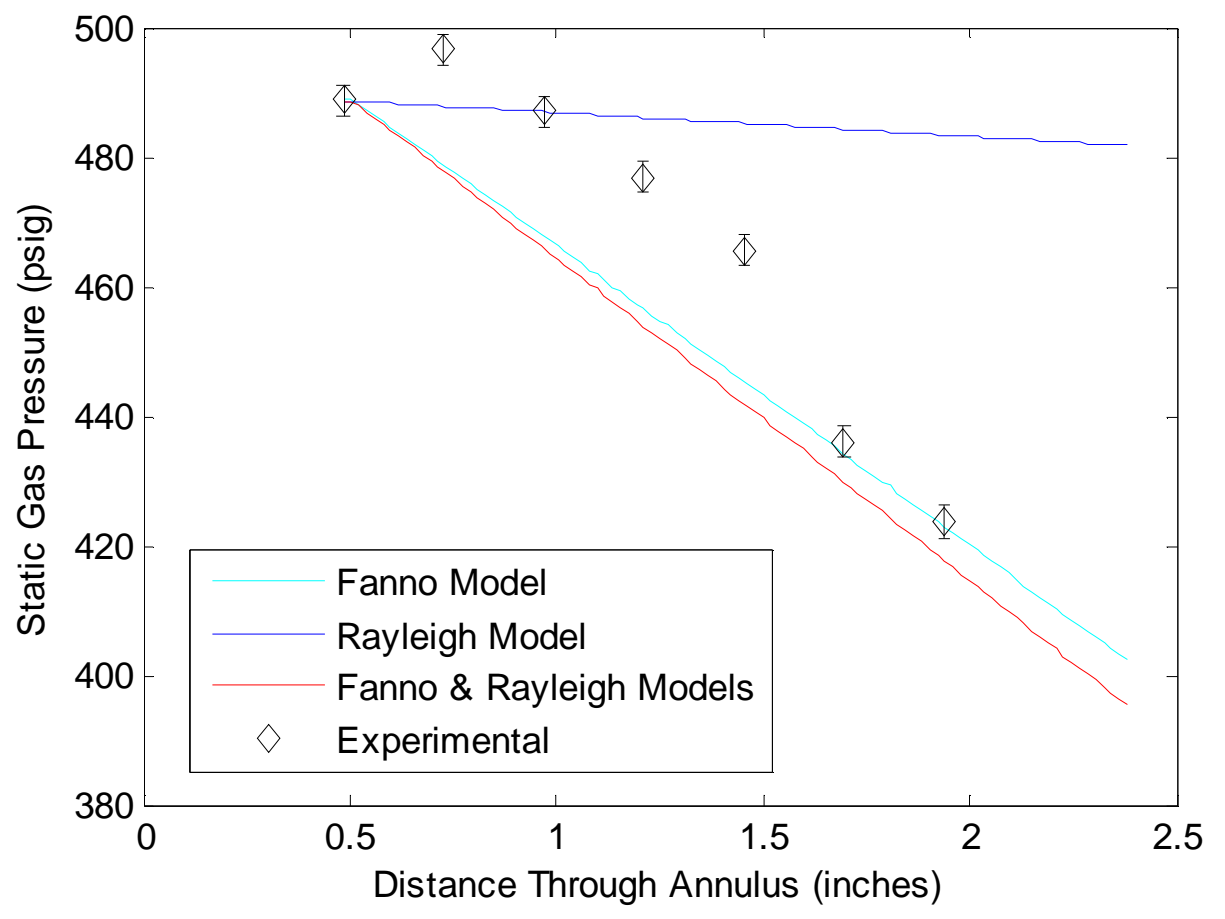

Figure 62. Helium test 2: predicted and measured average steady-state pressures 


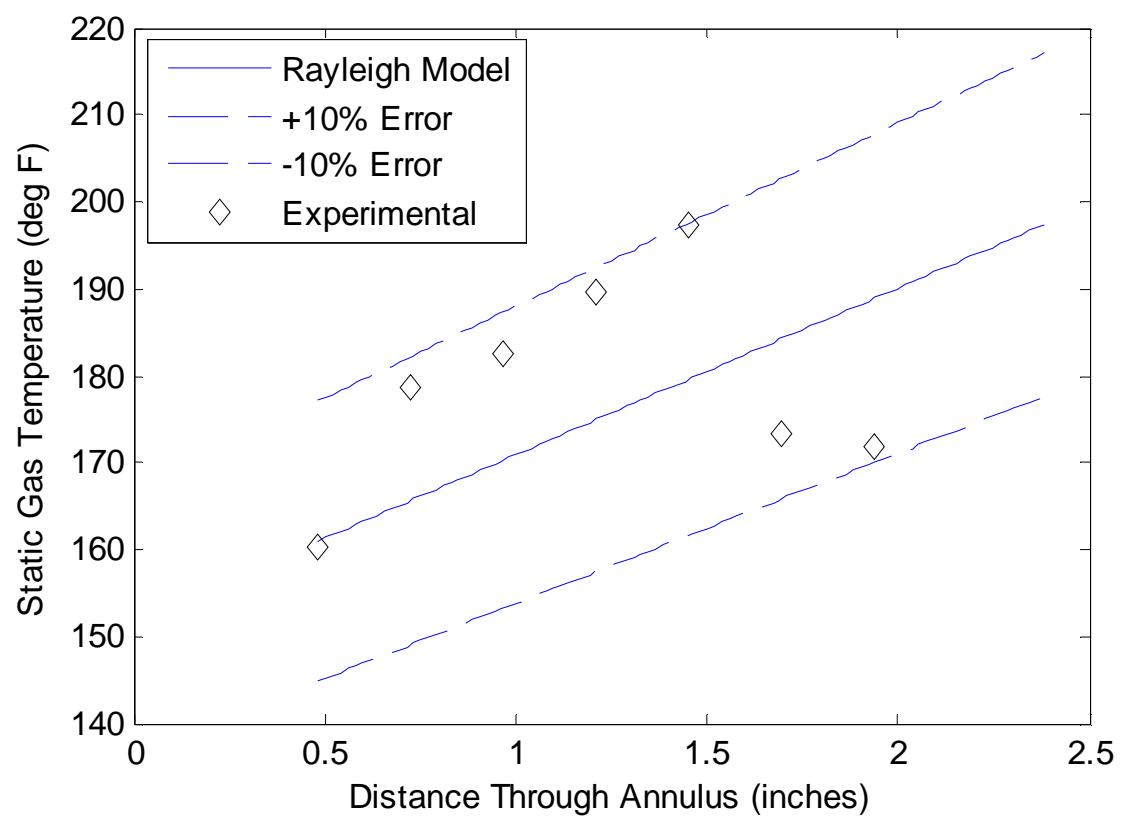

Figure 63. Helium test 2: predicted and measured average gas temperatures

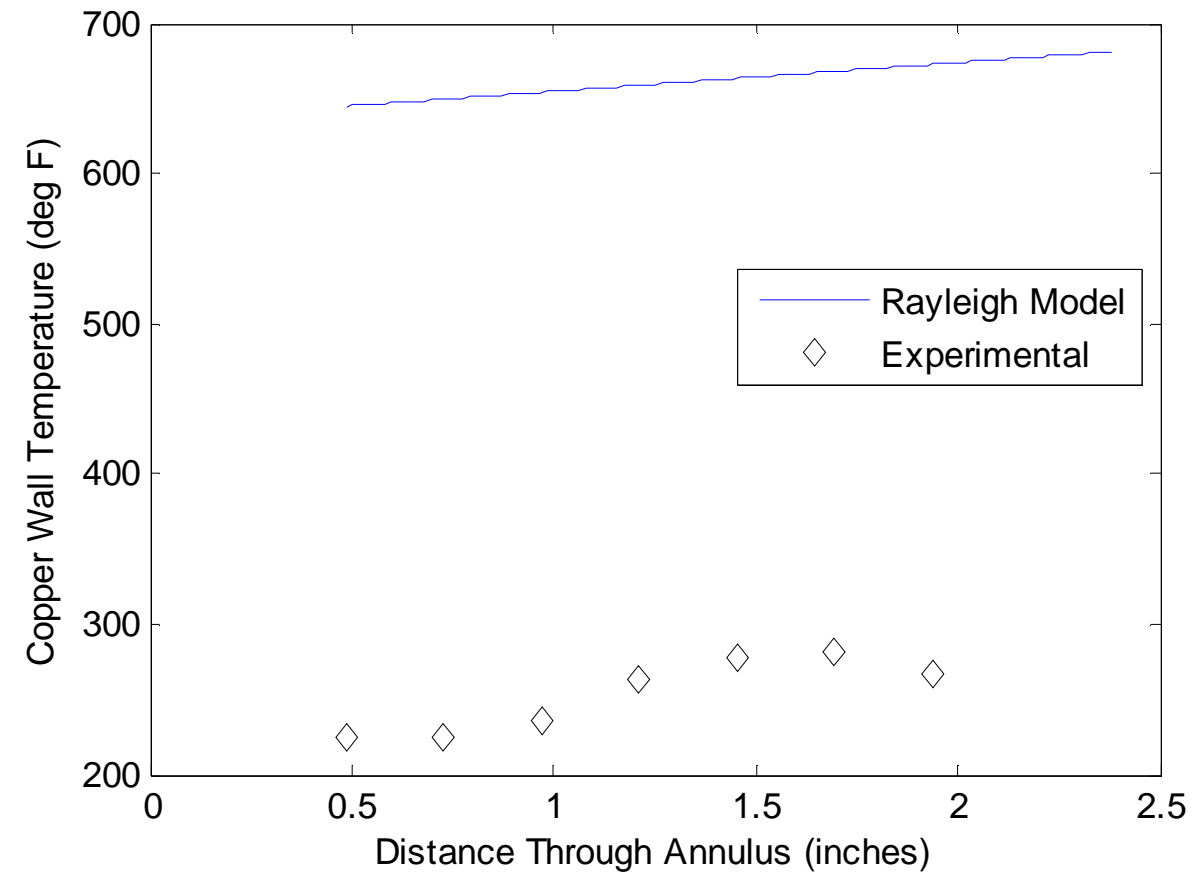

Figure 64. Helium test 2: predicted and measured steady state wall temperatures 


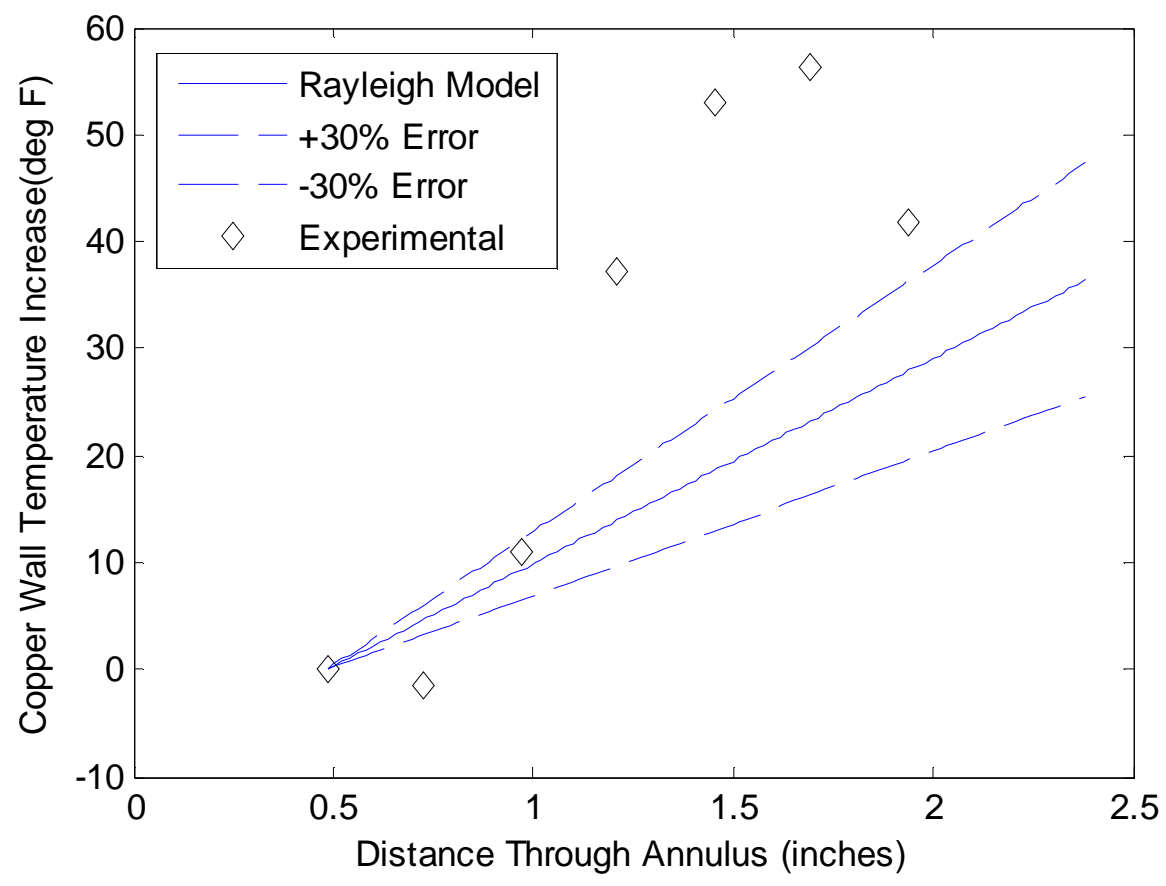

Figure 65. Helium test 2: predicted and measured copper wall temperature increase

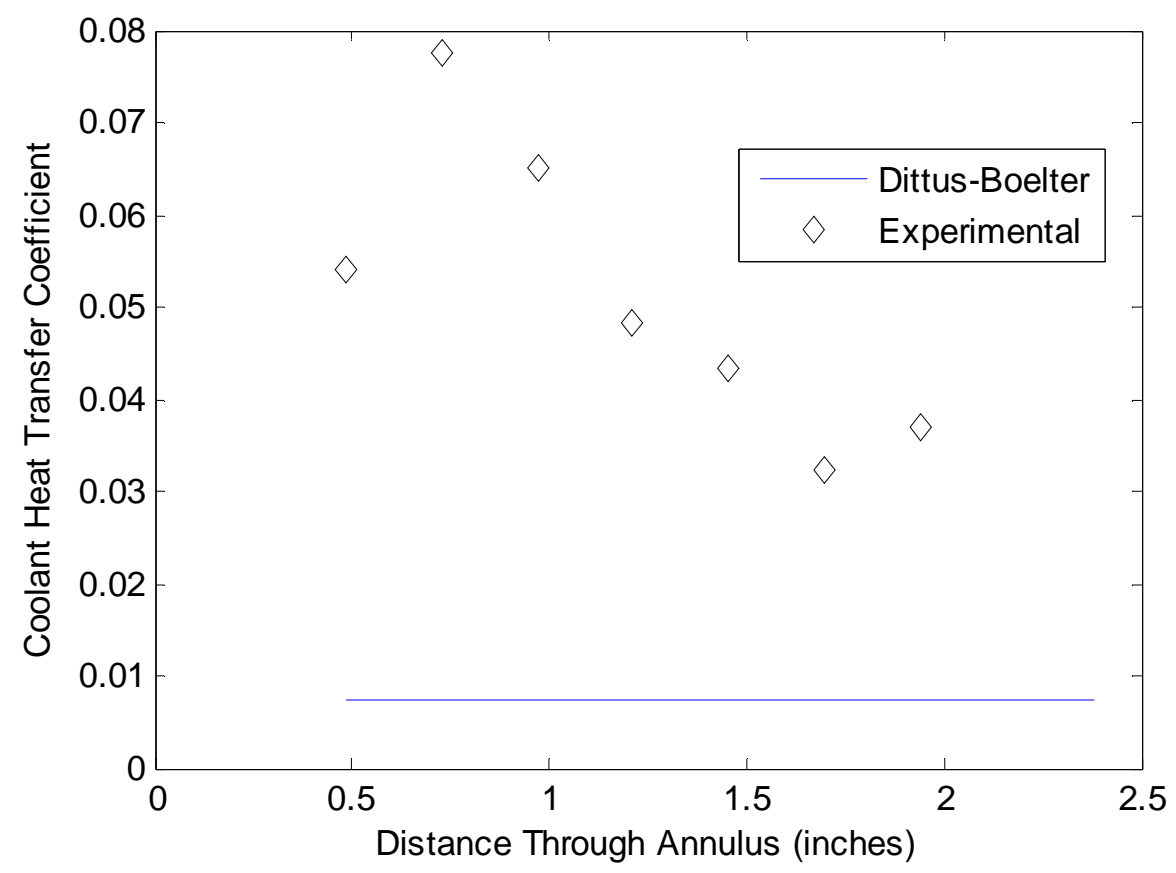

Figure 66. Helium test 2: predicted and experimental coolant heat transfer coefficients 


\section{III.2.8 Ideal Gas Results Discussion}

For the most part, the Rayleigh and Fanno analytical models match the helium experimental data well. Nevertheless, there are some discrepancies that should be discussed. Of primary interest are the: (1) decreased pressures at the inlet and outlet of the annulus, (2) elevated predicted copper temperatures as compared to measured, and (3) non-linearity of the copper wall temperature increase. Each of these topics will be discussed separately in the following sections.

\section{III.2.8.1 Pressure Discrepancies}

In both tests, abnormally low pressures were experienced at the inlet and outlet of the annulus (refer to Figure 53 and Figure 62). One possible explanation for this phenomenon is the presence of recirculation zones due to the abrupt changes in flow path at the inlet and outlet of the annulus. To check this theory, the pressure and temperature measured at the second port in the annulus were input into the Rayleigh and Fanno models since this port is presumably located outside of the recirculation zones. The relevant results are shown in the figures below:
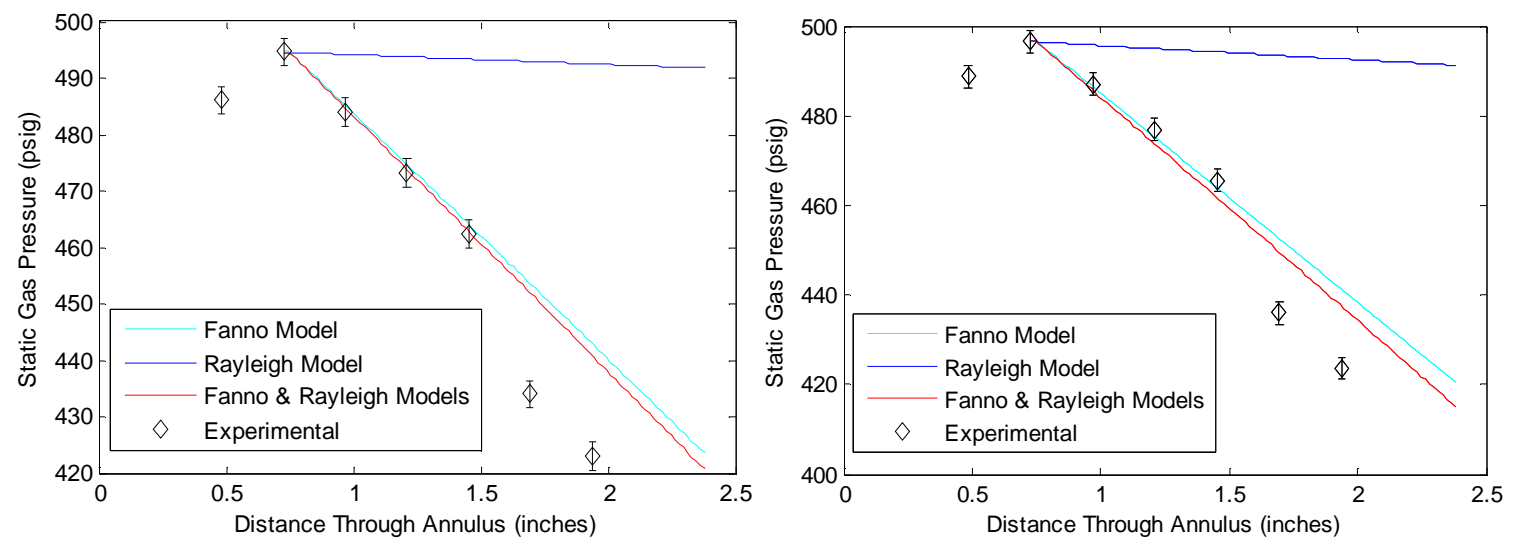

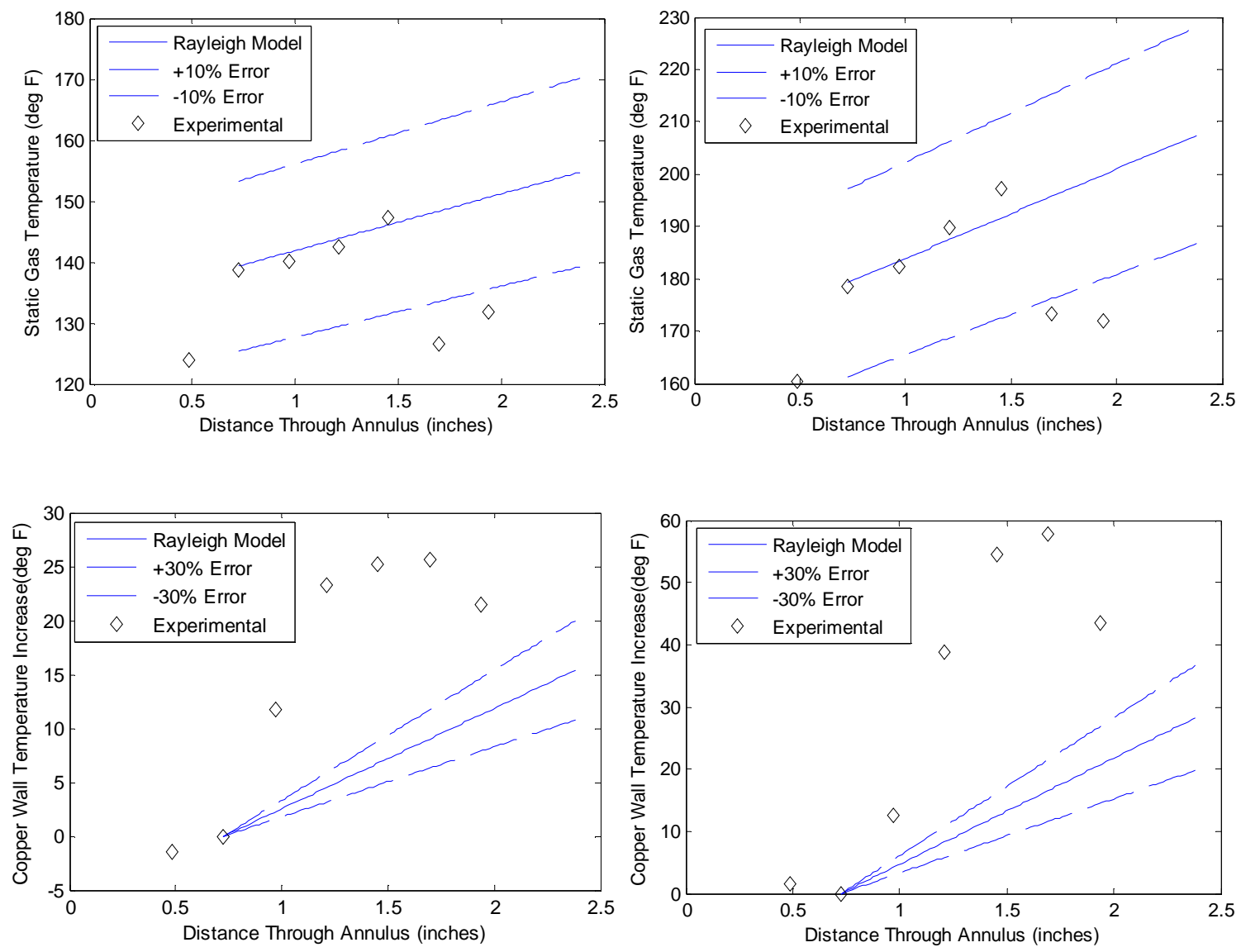

Figure 67. Nitrous oxide cooled hot fire results (test 1 on the left, test 2 on the right)

\section{III.2.8.2 Copper Temperature Magnitude Discrepancies}

The most striking difference between the experimental results and the model predictions is with the copper wall temperatures. For both experiments, the model over predicts copper temperatures by more than $200^{\circ} \mathrm{F}$. There are several factors that may contribute to this temperature difference. First, the constant copper temperature assumption may not be sufficiently accurate. The Biot number analysis of data from previous tests found that the physical copper temperature distribution more closely mimicked the constant copper temperature scenario than the variable one. However, they did not indicate that there was 
no temperature variation within the copper because the Biot numbers were not significantly less than 0.01 . The copper temperatures are currently monitored in a flange relatively far away from the coolant and combustion gases (as shown in Figure 68) one of the coolest locations within the copper. In the finite element model discussed in Section III.2.4, it was shown that temperature gradients as high as $130^{\circ} \mathrm{F}$ may exist between these two locations for some firing conditions. This is shown in Figure 68. It should be noted that the contour plot below is not specific to either of the helium runs. Based on this plot, it is reasonable to consider that the copper temperatures measured in the flange are not indicative of the copper temperatures closer to the coolant and combustion gases that are predicted by the Rayleigh model.

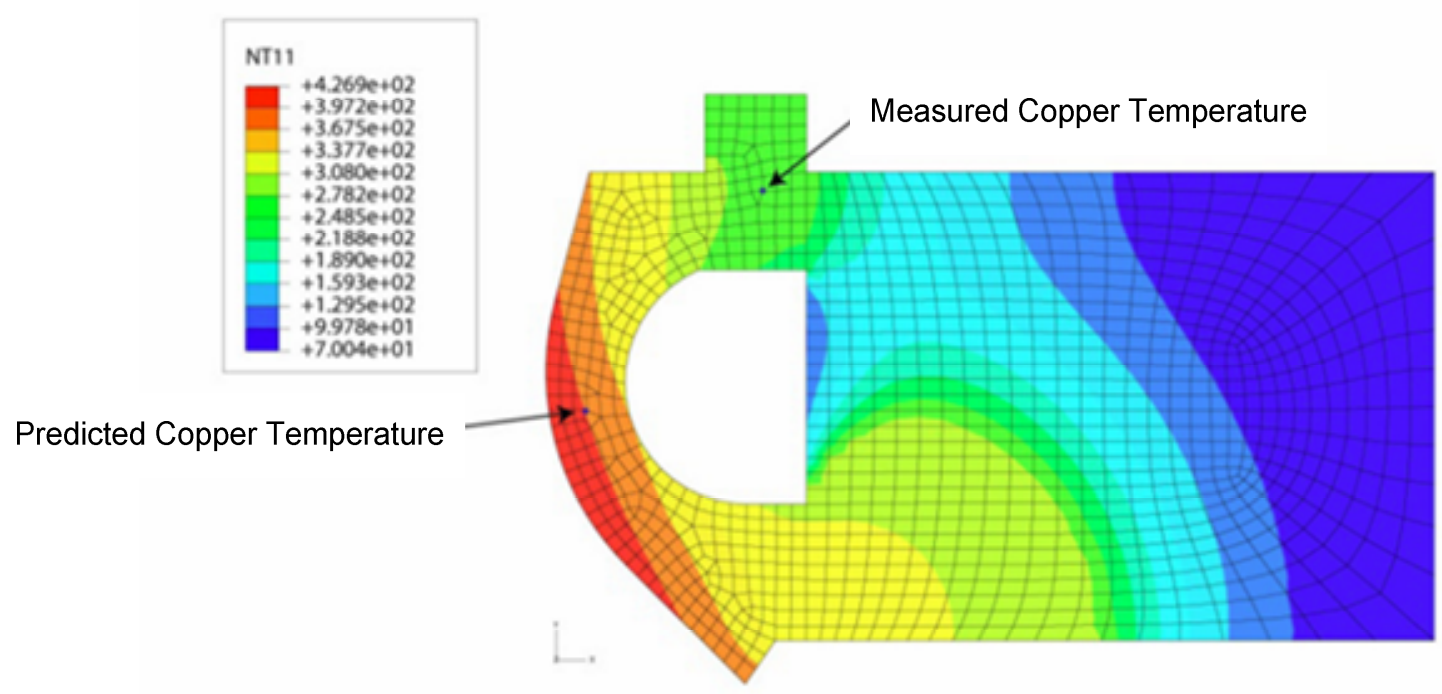

Figure 68. Radial copper temperature distribution in degrees Fahrenheit(17) 
While the variable cooper temperature provides the most obvious explanation for the unusually high predicted copper temperatures, there are other factors that may contribute to this. Most notably, the heat transfer coefficient derived from the Petukhov Nusselt number correlation assumed fully developed flow. Heat transfer coefficients are significantly higher in entrance regions and it was discussed in section III.2.4 that up to $50 \%$ of the annulus may be considered an entrance region. By this logic, the copper temperatures predicted by the Petukhov correlation will likely under predict heat transfer coefficients (especially in the beginning of the annulus) and therefore over predict wall temperatures. Also recall that the hot side heat transfer coefficient was assumed constant through the nozzle at the throat value. Because the heat transfer coefficient peaks at the throat and sharply drops of longitudinally before and after the throat, the heat transfer coefficient and, consequently, the heat transfer from the combustion gases is probably overestimated.

\section{III.2.8.3. Copper Temperature Nonlinearity}

A relatively linear copper wall temperature distribution through the annulus is expected by the Rayleigh model. This was not observed in the measured data (see Figure 55and Figure 64). A better view of the experimental data is shown in Figure 69 and Figure 70. 


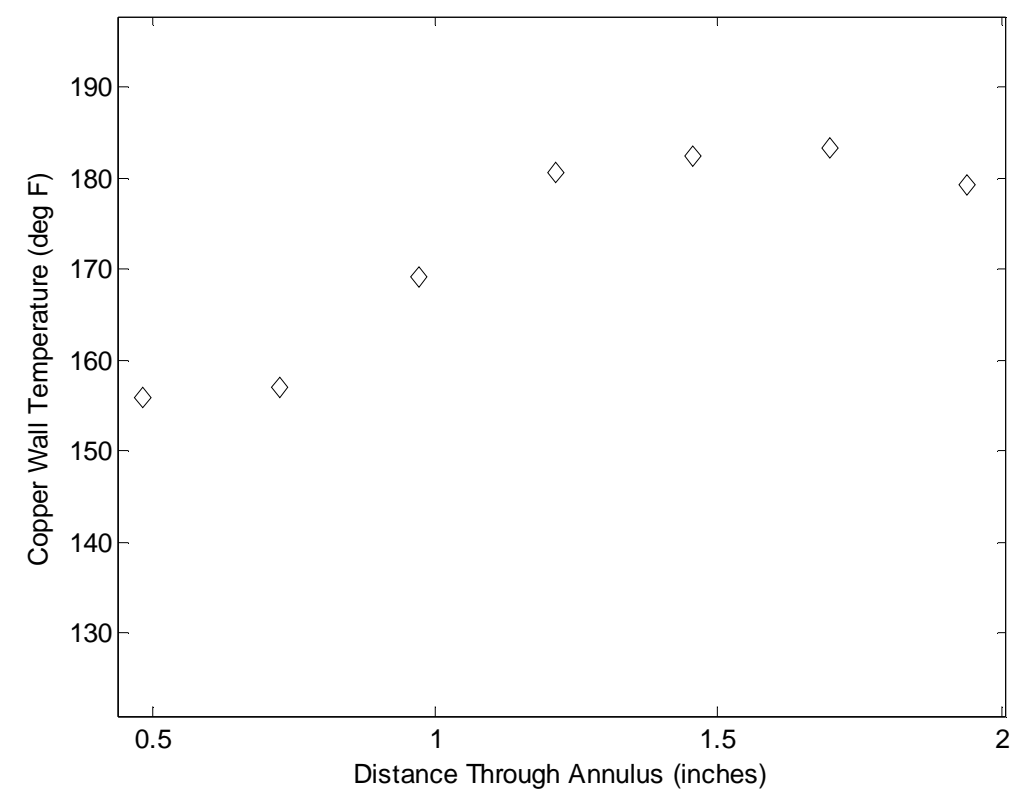

Figure 69. Helium test 1: experimentally measured copper temperature

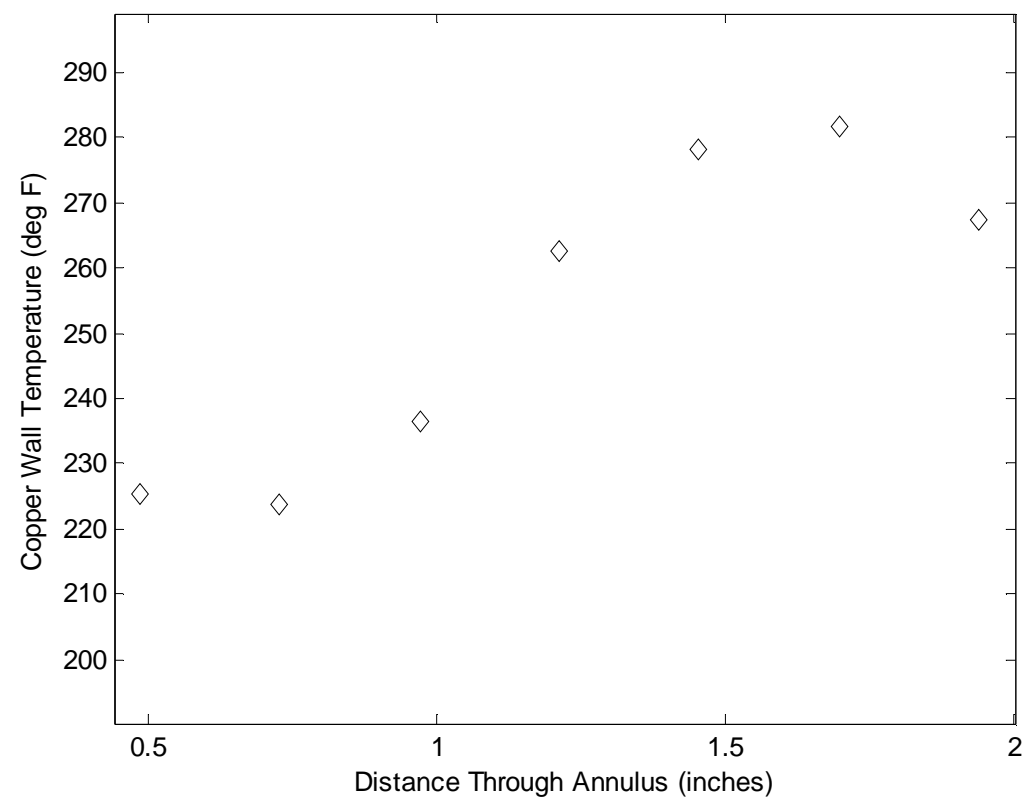

Figure 70 . Helium test 2 : experimentally measured copper temperature 
A primary cause of this discrepancy is the annulus geometry of the insert. Because the inlet and outlet ends of the annulus are physically connected, though separated via a copper fin, heat is allowed to conduct through the copper, allowing additional cooling capacity to the end of the annulus. This phenomena is not predicted in the model because the annulus is modeled as a straight pipe and conduction through the copper is not accounted for.

\section{III.2.9 Ideal Gas Conclusions}

The most important point to be made from the ideal gas tests is that, with the exception of copper temperatures, the data collected by the Rayleigh instrumentation is relatively predictable. The trends seen in both gas pressure and temperature matched the combined Rayleigh-Fanno model with minimal error. Secondly, it was observed that the first and last two instrumentation ports may be located in entrance and exit regions and may not be useful with models that do not predict these entrance and exit phenomena. The location

of the copper thermocouples in the nozzle is likely in a low temperature region. It may be useful to use the FEM developed to "map" measured temperatures to locations closer to the coolant and combustion gases.

Another important milestone that was achieved with these tests was identifying errors in and improving the instrumentation. These achievements include but are not limited to: (1) installing and calibrating a flow meter to improve the accuracy of mass flow rate measurements in future tests, and (2) identification and resolution of ground looping due 
to improper thermocouple wiring. Because of these improvements, additional confidence can be placed in the data collected from the two-phase $\mathrm{N}_{2} \mathrm{O}$ Rayleigh tests.

\section{IV: TWO-PHASE COOLANT MODELING AND EXPERIMENTS}

Two different models are initially developed in an attempt to characterize the flow of two-phase nitrous oxide around the nozzle throat. The first is a simple, one-dimensional model in which the properties of the fluid are averaged over each cross section of the annulus. This model is termed the "homogeneous flow model." Secondly, a more advanced separated flow model with interface exchange is developed to predict the location of dryout. This model is fittingly named the "Revellin-Thome model" after its founders. The Shah correlation is used in conjunction with both models in the nucleate boiling regime. Unfortunately no heat transfer coefficient correlations exist that are applicable to nitrous oxide in the mist flow regime (post-dryout). The Groeneveld correlation is discussed as a representative method of characterizing the heat transfer coefficients in the mist flow region but cannot be used to predict actual heat transfer coefficient as it is developed for steam-water mixtures only. The single phase Rayleigh equations and Dittus-Boelter correlation are used to model the fluid in the case that it reaches a superheated vapor. This is summarized in Table 7: 
Table 7. Modeling technique as a function of flow regime

\begin{tabular}{|c|c|c|c|}
\cline { 3 - 4 } & & \multicolumn{2}{|c|}{ Modeling Technique } \\
\hline Quality & Flow Type & Hydrodynamic & Heat Transfer \\
\hline$x_{0}<x_{\text {do }}$ & Pre-Dryout & Revellin-Thome Model & Shah Correlation \\
\hline$x_{\text {do }}<x<1$ & $\begin{array}{c}\text { Post-Dryout, } \\
\text { Pre-Superheat }\end{array}$ & Homogeneous Flow Model & $\begin{array}{c}\text { Empirically Based Correlation } \\
\text { (Not Developed Here) }\end{array}$ \\
\hline$x>1$ & Post-Superheat & Rayleigh Equations & $\begin{array}{c}\text { Single Phase Dittus- } \\
\text { Boelter Correlation }\end{array}$ \\
\hline
\end{tabular}

\section{IV.1 PRELIMINARY MODELING AND PROBLEM SETUP}

It is first necessary to summarize simplifying assumptions regarding how the system as a whole will be modeled. First, by the Biot analysis in chapter II, the copper is assumed to be of uniform temperature through the thickness and circumferentially within each respective thermocouple section. The heat flux is assumed constant. Body forces are neglected, allowing the annulus to be "unwound" into a straight configuration. This is depicted in Figure 71.

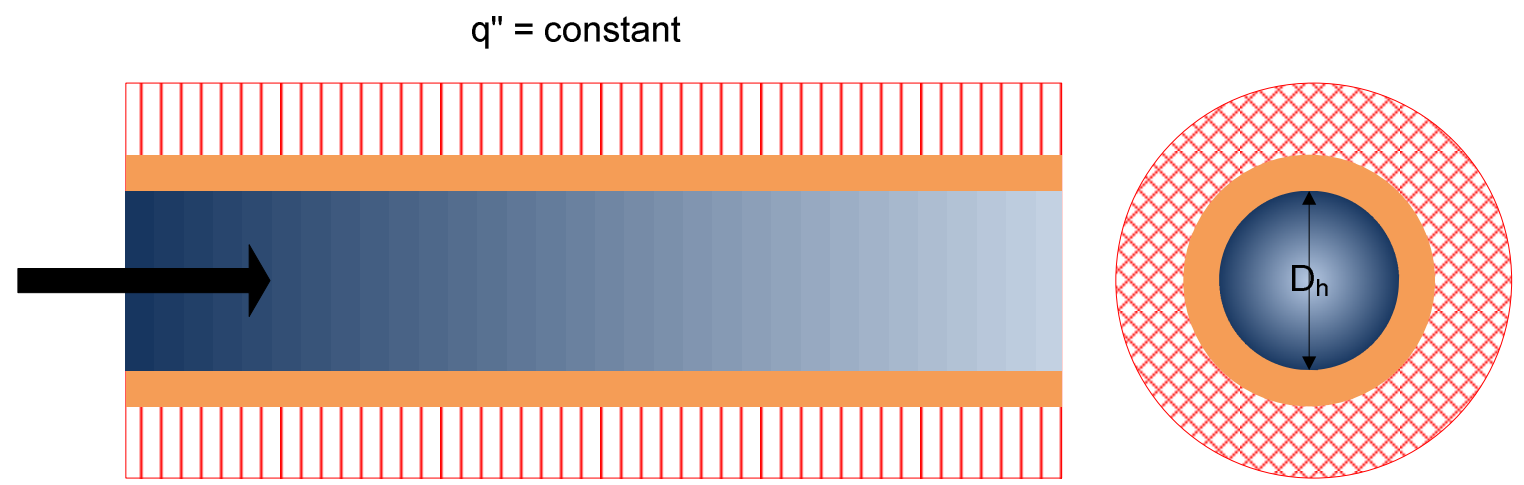

Figure 71. Simplified model of two-phase Rayleigh flow 
Friction is neglected in all but the Revellin-Thome models. The annulus is assumed round with a diameter equal to its hydraulic diameter. In this case, the hydraulic diameter is approximately 0.1295 inches. The uniform heat flux $q$ ” is given by

$$
q^{\prime \prime}=\frac{q}{A_{\text {cold }}}
$$

where $A_{\text {cold }}$ is the area of copper exposed to the coolant and $q$ is the total convective heat transfer rate from the hot side. Note that if the annulus is assumed round, $A_{\text {cold }}$ can be approximated as the product of the perimeter $P_{h}$ and the duct length $L$. Finally, in all subsequent two-phase models, the assumption of thermal equilibrium is made.

\section{IV.1.1 Fluid Properties}

There is a shortage of tabulated fluid properties of nitrous oxide likely because of its volatile nature at high temperatures. While the specific heat is available as a function of temperature from various sources (18) (19), there is limited information regarding the thermal conductivity and dynamic viscosity. In order to develop a two-phase model, it is imperative that separate liquid and gas saturation properties be available. An extensive literature search suggested that, with the exception of very expensive software packages (20), no source of such data for nitrous oxide exists. 
Carbon dioxide has been shown to behave very similarly to nitrous oxide due to similarities in molecular structure (21). A full set of material properties is available over a large range of temperatures for carbon dioxide (19). Based on this, the possibility of using the physical properties of carbon dioxide in place of those of nitrous oxide was investigated. The specific heat of nitrous oxide has been studied and tabulated data is readily available (18). This data was compared to that of carbon dioxide over a range of temperatures. The results are shown in Figure 72 below.

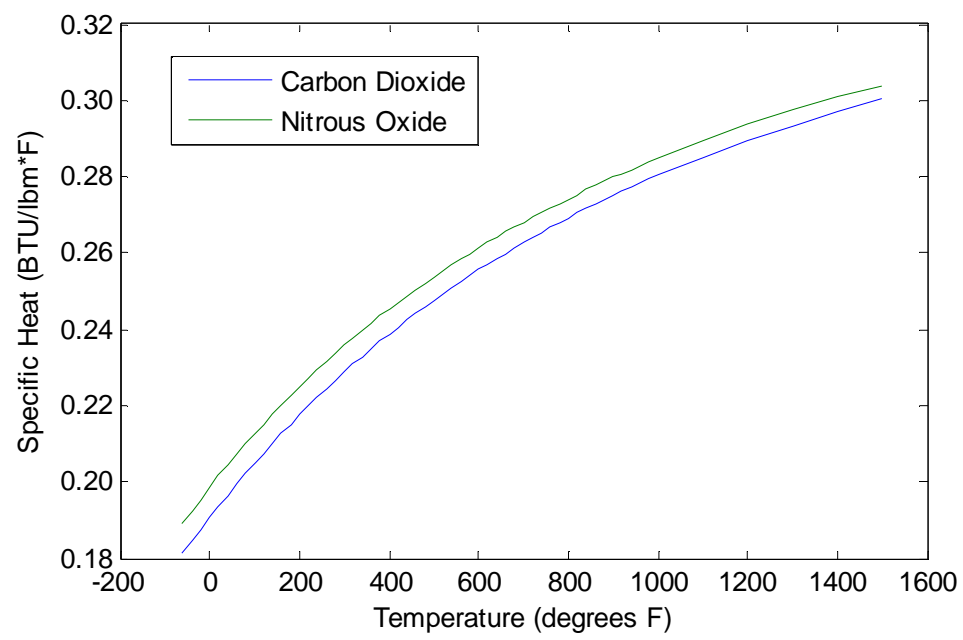

Figure 72. Similarity of specific heats of $\mathrm{CO}_{2}$ and $\mathrm{N}_{2} \mathrm{O}$

The percent difference between the two values is shown in Figure 73 below. In the temperature range for which this analysis was performed (approximately 0 to $100^{\circ} \mathrm{F}$ ) the average percent difference between the nitrous oxide and carbon dioxide specific heats is 3.8\%. Considering the number of assumptions that have been made thus far and the advantage offered by a complete set of fluid property data, this amount of error may be considered acceptable. 


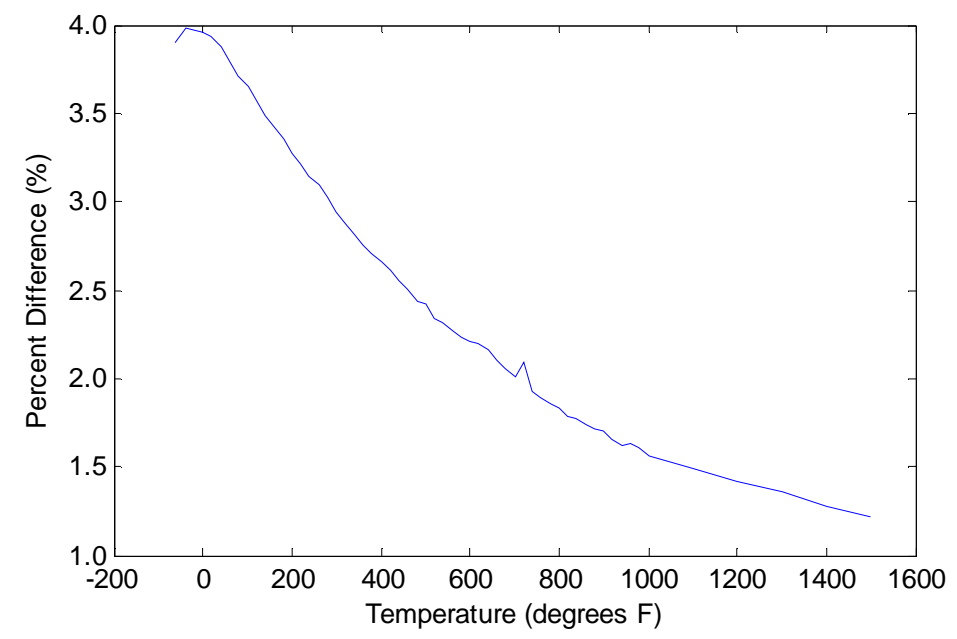

Figure 73. Percent difference between specific heats of $\mathrm{CO}_{2}$ and $\mathrm{N}_{2} \mathrm{O}$

It is reasonable to assume that similar trends would be observed for the thermal conductivity and dynamic viscosity. While it is not ideal, it is necessary to use the thermal properties (specific heat, thermal conductivity and dynamic viscosity) of carbon dioxide in all subsequent nitrous oxide models.

\section{IV.1.2 System Schematic and Description}

A schematic of the nitrous oxide cooling system including only the relevant components is shown in Figure 74 below. A pressurized tank of liquid $\mathrm{N}_{2} \mathrm{O}$ is attached to an injector through a line and a number of fittings that are assumed to have no major effects on the system. On the other side of the inlet injector is the annulus through which the nozzle is cooled. Finally, a second injector is located at the end of the annulus. The outlet injector exhausts to ambient conditions. 


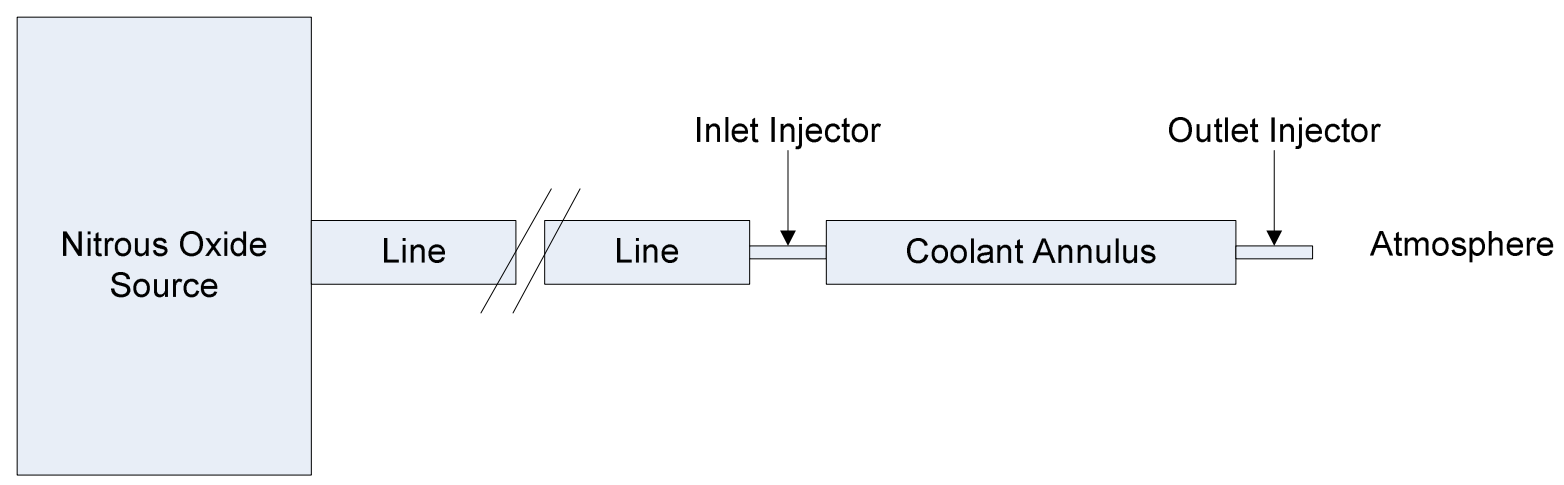

Figure 74. Coolant system schematic

The process across the injectors is assumed isenthalpic. The nitrous oxide enters the inlet injector to the annulus as a saturated liquid at a known pressure $P_{\text {line }}$. Because these two properties (pressure and saturated liquid) of the fluid are known, all other characteristics of the flow (i.e. enthalpy, entropy, density, temperature) at this point are also known. The pressure at the inlet of the annulus is measured and will therefore be taken as a known quantity. The throttling process across the first injector is shown on the Mollier diagram in Figure 75.

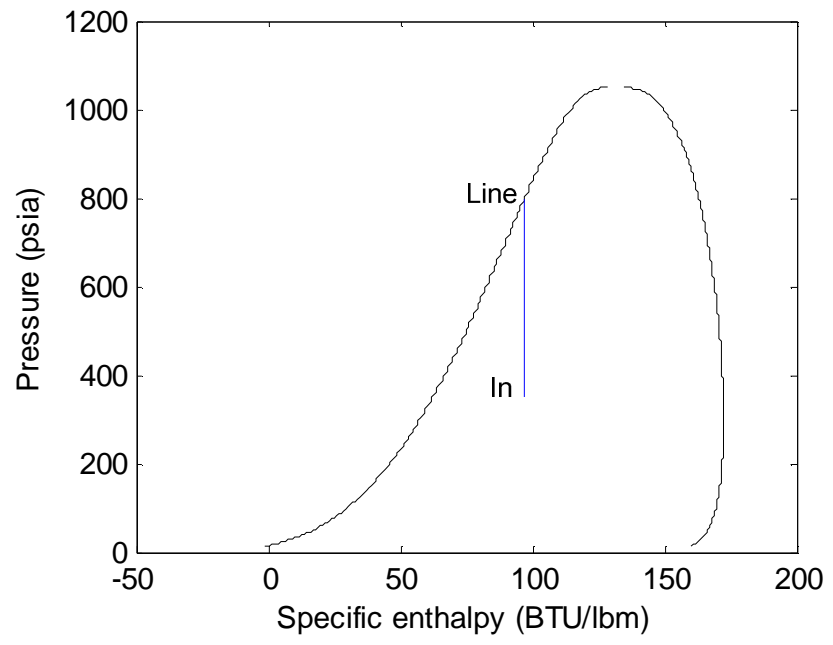

Figure 75. Isenthalpic throttling process across inlet injector 
Because of the isenthalpic injector assumption, the enthalpy of the fluid after exiting the injector is known. Note that this is actually a lower bound on inlet quality. In reality, some heat will be transferred into the fluid as it flows through the injector, resulting in a increase in enthalpy (and thus quality) with the pressure decrease. For the purpose of this analysis, the lower bound on inlet quality will be used. Now, because the pressure and enthalpy at the annulus inlet are defined, the thermodynamic quality can be determined at this point by

$$
x_{i n}=\frac{h_{\text {in }}-h_{v}\left(P_{\text {in }}\right)}{h_{l}\left(P_{\text {in }}\right)-h_{v}\left(P_{\text {in }}\right)}
$$

The pressure, enthalpy and quality at the inlet of the annulus are used as inputs into the two-phase models developed in the following sections.

\section{IV.2 Homogeneous Flow Model}

One of the simplest and most common two-phase models is the homogeneous flow model in which the properties of the fluid are averaged over each cross section of the annulus. In this way, a one-dimensional flow assumption is made allowing single phase fluid dynamic principles to be applied. This concept is summarized in Figure 76 below: 

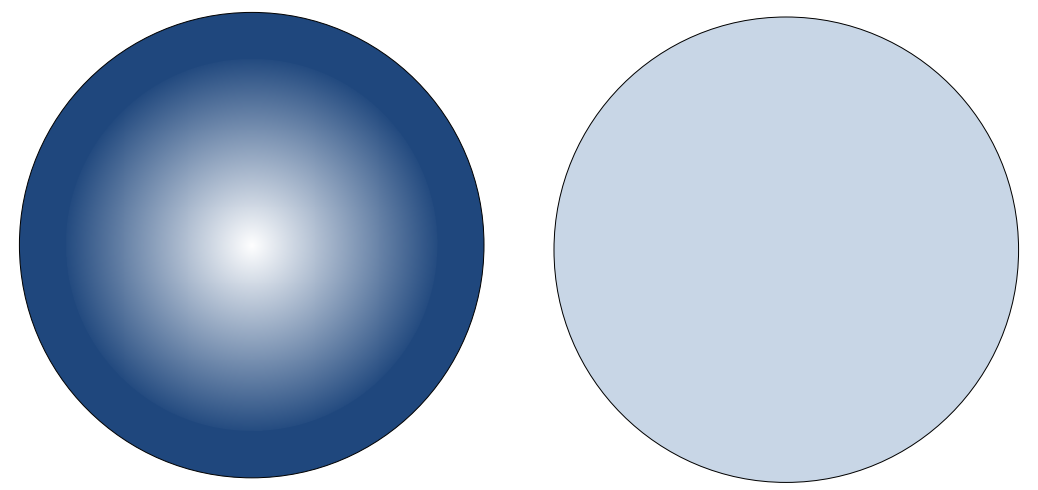

Figure 76. Annulus cross-section with (right) and without (left) 1-D flow assumption

In addition to this, the homogeneous flow model assumes that the two-phases are in thermal equilibrium and move at the same speed (i.e. the slip ratio is unity). Several sources recommend that a homogeneous flow model be one of the first developed in most two-phase flow systems as it is relatively simple to apply and can give good first approximations (5)(22). The following section outlines the model developed for the system of interest.

The mass-averaged density of the flow at the inlet is simply a function of the pressure and quality at the inlet and is given by:

$$
\frac{1}{\rho_{i n}}=x_{i n}\left[\frac{1}{\rho_{v}\left(P_{i n}\right)}-\frac{1}{\rho_{l}\left(P_{i n}\right)}\right]+\frac{1}{\rho_{l}\left(P_{i n}\right)}
$$

Assuming one-dimensional steady state flow, and neglecting body, shear forces and heat generation, the differential forms of conservation of mass, momentum and energy 
equations governing the homogeneous two-phase flow in the heated annulus are given by equations (4.4), (4.5) and (4.6) respectively.

$$
\begin{gathered}
\frac{\partial}{\partial z}\left(\rho_{T P} u_{T P} A\right)=0 \\
\frac{\partial}{\partial z}\left(\rho_{T P} u_{T P}^{2} A\right)=-A \frac{\partial P}{\partial z} \\
\frac{\partial}{\partial z}\left(\rho_{T P} u_{T P} A h_{T P}\right)+\frac{1}{2} \frac{\partial}{\partial z}\left(\rho_{T P} u_{T P}^{2} A\right)=P_{h} q^{\prime \prime}
\end{gathered}
$$

where $z$ is the distance from the annulus inlet, $h_{T P}$ is the mixture enthalpy, $P$ is the mixture pressure, $u_{T P}$ is the mixture velocity, $P_{h}$ is the heated perimeter of the annulus and $q$ " is the constant heat flux applied to passage. Equation (4.4) simplifies to

$$
\rho_{T P} u_{T P} A=\text { constant }=\dot{m}
$$

where $A$ is the cross sectional area of the annulus and is constant in this case. Then, the two-phase mass flux is also a constant and is given by

$$
G=\rho_{T P} u_{T P}=\frac{\dot{m}}{A}=\text { constant }
$$

Substituting this into equation (4.5) and simplifying gives 


$$
G \frac{\partial u_{T P}}{\partial z}=\frac{\partial P}{\partial z}
$$

Integrating this with respect to $z$ between arbitrary locations (1) and (2) results in

$$
G\left(u_{T P_{2}}-u_{T P_{1}}\right)=\left(P_{2}-P_{1}\right)
$$

Finally, rearranging equation (4.8) and substituting it in for $u_{T P 1}$ and $u_{T P 2}$ in the above equation gives

$$
G^{2}\left(\frac{1}{\rho_{T P_{1}}}-\frac{1}{\rho_{T P_{2}}}\right)=\left(P_{2}-P_{1}\right)
$$

Substituting continuity into equation (4.6) gives:

$$
\frac{\partial h_{T P}}{\partial z}+\frac{1}{2} \frac{\partial u_{T P}}{\partial z}=\frac{P_{h} q^{\prime \prime}}{\dot{m}}
$$

Integrating the above equation with respect to $z$ between arbitrary locations (1) and (2) gives

$$
h_{T P_{2}}-h_{T P_{1}}=\frac{P_{h} q^{\prime \prime} Z}{\dot{m}}-\frac{1}{2}\left(u_{T P_{2}}{ }^{2}-u_{T P_{1}}{ }^{2}\right)
$$

Notice that this result is identical to that derived for single phase Rayleigh flow in Chapter II. This is expected since the homogeneous model essentially treats the two- 
phase flow as if it were a single phase with properties averaged across the annulus cross section. The method of solution of the above equations is nearly identical to that of the ideal gas model. The annulus is discretized into equally sized increments. Starting at the inlet to the annulus where the state of the fluid is fully defined, equations (4.8), (4.11) and (4.13) are solved by stepping through the annulus and solving for the properties at each location $z$ based on those at the previous location $z-d z$. This requires an iterative process and was therefore best performed with MATLAB (see Appendix B). Note that this is not a numerical approximation as the solution method would suggest but a closed form solution. The energy equation is first solved assuming negligible changes in kinetic energy (i.e. $u_{z}=u_{z-d z}$ ):

$$
h_{T P_{z}} \approx h_{T P_{z-d z}}+\frac{d q}{\dot{m}}
$$

where $d q$ is the heat transfer rate (in BTU/sec) applied to any given increment and is calculated by multiplying the total heat transfer rate by the ratio of the length of a discretized increment to the total length of the annulus:

$$
d q=\left(\frac{d z}{L}\right) q
$$

The quality at location $z$ is then given by 


$$
x_{z}=\frac{h_{T P_{z}}-h_{v}\left(P_{i n}\right)}{h_{l}\left(P_{i n}\right)-h_{v}\left(P_{i n}\right)}
$$

Notice that the saturated liquid and vapor properties are evaluated at the inlet pressure throughout the annulus despite the pressure drop. By assuming the gas and liquid velocities are equal and equating equations (3.6) and (3.7), the void fraction at location $z$ is given by

$$
\alpha_{z}=\frac{\rho_{l} x_{z}}{\rho_{l} x_{z}+\rho_{g}\left(1-x_{z}\right)}
$$

The density at state $z$ is then given by

$$
\frac{1}{\rho_{T P_{z}}}=x_{z}\left[\frac{1}{\rho_{v}\left(P_{i n}\right)}-\frac{1}{\rho_{l}\left(P_{i n}\right)}\right]+\frac{1}{\rho_{l}\left(P_{i n}\right)}
$$

The velocity at state $z$ is then derived from continuity and is given by

$$
u_{T P_{Z}}=\frac{G}{\rho_{T P_{Z}}}
$$

Finally, the energy equation can be reevaluated using the velocity predicted by equation (4.19) above to account for changes in kinetic energy: 


$$
h_{z}=h_{i n}+\frac{q_{i n-z}}{\dot{m}}-\frac{1}{2}\left(u_{z}^{2}-u_{i n}^{2}\right)
$$

Equations (4.8), (4.11) and (4.13) are then reevaluated to give an updated velocity at state $z$. This process is repeated until the change in enthalpy at state $z$ is less than $10^{-4}$. After the density, enthalpy, velocity and quality at state $z$ are fixed, the pressure is evaluated from the Rayleigh line equation:

$$
P_{z}=P_{z-d z}+G^{2}\left(\frac{1}{\rho_{T P_{z}-d z}}-\frac{1}{\rho_{T P_{z}}}\right)
$$

Because thermal equilibrium was assumed, the temperature at state $z$ is given by the saturation temperature corresponding to $P_{z}$ :

$$
T_{z}=T_{\text {saturation }}\left(P_{z}\right)
$$

There is significant controversy over how the fluid properties of the system should be defined in homogeneous flow models. Some authors recommend using complicated empirical correlations, others recommend simple mass or area averaged definitions. Because the defining assumption of this model is one-dimensional flow, an area averaged method makes intuitive sense and was therefore chosen. Another argument for this method is that it gives values that approach those of the liquid and vapor as the quality approaches zero and one respectively. The two-phase thermal conductivity, dynamic 
viscosity and specific heat are then given by equations (4.23), (4.24) and (4.25) below and are evaluated at each $z$ location:

$$
\begin{gathered}
k_{T P}=k_{L}(1-\alpha)+k_{g} \alpha \\
\mu_{T P}=\mu_{L}(1-\alpha)+\mu_{g} \alpha \\
C_{P_{T P}}=C_{P_{L}}(1-\alpha)+C_{P g} \alpha
\end{gathered}
$$

Based on the previous analysis, it may seem that a single phase convective correlation could be used to model the heat transfer properties of the homogeneous two-phase fluid. However, this is not the case as a convective heat transfer correlation would not account for the microscopic heat transfer (i.e. bubble nucleation and growth). Thus, using such a correlation would grossly underestimate the amount of heat transfer between the wall and the coolant. Either a more advanced correlation must be used that accounts for the latent heat of vaporization associated with the phase change, or it must be conceded that the homogeneous flow model is only capable of modeling hydrodynamic properties of the flow. This will be discussed further in the next section.

\section{IV.2.1 Shah Correlation}

Because of its wide range of applicability, the Shah correlation was chosen to model the heat transfer coefficients in conjunction with the homogeneous flow model. A similar approach was taken in which properties at each discrete $z$ location were determined. First, it is necessary to calculate the three major dimensionless parameters on which this model is based. The boiling number is given by 


$$
B o=\frac{q}{A_{\text {cold }} G\left(h_{v}-h_{l}\right)}
$$

where $q$ is the heat transfer rate from the hot side, $A_{\text {cold }}$ is the area of copper exposed to the coolant, $G$ is the constant mass flux, and $h_{v}$ and $h_{l}$ are the specific enthalpies of the saturated vapor and saturated liquid respectively. The Convection number is

$$
\operatorname{Co}=\left(\frac{1}{x}-1\right)^{0.8}\left(\frac{\rho_{v}}{\rho_{l}}\right)^{0.5}
$$

where $x$ is the thermodynamic quality given by equation (4.16) from the homogeneous model, and $\rho_{v}$ and $\rho_{l}$ are the saturated vapor and saturated liquid densities respectively. Note that because the quality is varying through the annulus so too will the Convection number (i.e. $\operatorname{Co}=\operatorname{Co}(z))$. Finally, the Froude number is given by

$$
F r_{L}=\frac{G^{2}}{\rho_{L}^{2} g D_{H}}
$$

which represents the ratio of inertial to gravitational forces. In this case, a high Froude number indicates that the gravitational forces are negligible, or that the flow will not be stratified. Typical operating conditions for the coolant system of interest are a mass flux of $470 \mathrm{lbm} / \mathrm{ft}^{2} \sec \left(2300 \mathrm{~kg} / \mathrm{m}^{2} \mathrm{sec}\right)$, a saturation pressure of 350 psia (24 bar), and a heat transfer rate of 4.3 BTU/sec. The Boiling, Convection and Froude numbers corresponding to these operating conditions are approximately $0.018,0.4$ and 195 respectively. 
The liquid convective heat transfer coefficient is given by a modified Dittus-Boelter correlation:

$$
h_{\text {liquid }}=0.023 \operatorname{Re}_{L}^{0.8} \operatorname{Pr}_{L}^{0.4} \frac{k_{L}}{D_{h}}
$$

where $D_{h}$ is the annulus hydraulic diameter, $k_{L}$ is the liquid thermal conductivity and $R e_{L}$ and $P r_{L}$ are the liquid Reynolds and Prandtl numbers. The liquid Reynolds number is given by

$$
R e_{L}=\frac{G(1-x) D_{h}}{\mu_{L}}
$$

and the liquid Prandtl number is given by

$$
\operatorname{Pr}_{L}=\frac{\mu_{L} C_{P_{L}}}{k_{L}}
$$

where $\mu_{L}$ and $C_{p L}$ are the molecular viscosity and specific heat of the liquid respectively. Four two-phase multipliers are now calculated. These multipliers represent the ratio of the two-phase fluid cooling capacity to that of the pure liquid: 


$$
\varphi \equiv \frac{h_{\text {cold }, T P}}{h_{\text {liquid }}}
$$

In this case, because the Froude number is larger than 0.4 and the boiling number is greater than 0.0003 , the nucleate boiling two-phase multiplier is given by

$$
\varphi_{n b}=230 B o^{0.5}
$$

The convective boiling two-phase multiplier is

$$
\varphi_{n b}=\frac{1.8}{N^{0.8}}
$$

where the value of $N$ is dependent on the value of the Froude number. For Froude numbers larger than 0.04 in horizontal annuli, $N$ is simply given by the Convection number:

$$
N=C o
$$

Substituting this into equation (4.35) gives the following two-phase convection multiplier:

$$
\varphi_{n b}=\frac{1.8}{C o^{0.8}}
$$


For values of $N$ greater than 1.0, the larger of the convective and nucleate boiling twophase multipliers is used. The first bubble suppression two-phase multiplier is given by

$$
\varphi_{b s_{a}}=F B o^{0.5} e^{2.74 N^{-0.1}}
$$

where for $B o$ greater than 0.0011 , the variable $F$ is equal to 14.7 . The second bubble suppression two-phase multiplier is given by

$$
\varphi_{b s_{b}}=F B o^{0.5} e^{2.47 N^{-0.15}}
$$

The appropriate two-phase multiplier for a given flow is dependent on the value of $N$ for that flow. The conditions are summarized in Table 8 below:

Table 8. Appropriate two-phase multiplier based on $N$

\begin{tabular}{|c|c|}
\hline $\mathrm{N}=\mathrm{Co}$ & Appropriate Two-Phase Multiplier \\
\hline Greater than 1.0 & Larger of convective boiling and nucleate boiling \\
\hline Between 0.1 and 1.0 & Bubble Suppresion A \\
\hline Less than 0.1 & Bubble Suppresion B \\
\hline
\end{tabular}

The two-phase convective heat transfer coefficient as predicted by Shah is then given by

$$
h_{\text {cold }}=\varphi h_{\text {liquid }}
$$


where the two-phase multiplier $\varphi$ for a given annulus location $z$ is chosen based on the criteria summarized in Table 8. Note that the two-phase multipliers and liquid heat transfer coefficients are functions of location in the annulus $z$. By extension, the cold side heat transfer coefficient will also be a function of $z$. Appropriately, these values are calculated at each location in the annulus. The wall temperature at location $z$ as predicted by the Shah correlation in conjunction with the homogeneous two-phase flow model is given by

$$
T_{\text {wall }_{z}}=\frac{q}{h_{\text {cold }} A_{\text {cold }}}+T_{z}
$$

where $T_{z}$ is given by equation (4.22).

\section{IV.2.2 Dryout Prediction}

In general, two-phase flows will dryout prior to reaching the saturated vapor line. This has been reported to occur over a wide range of qualities depending on individual flow characteristics and is illustrated in Figure 7 (8). One major limitation of the homogeneous flow model used by itself is that it has no mechanism for determining dryout other than assuming it occurs at the saturated vapor line. That is, the homogeneous flow model and Shah heat transfer correlation are applied to the system until they predict a quality of unity. As heat transfer coefficients are much higher in the nucleate boiling regime than the mist flow or film boiling regime, this method will likely over predict heat transfer coefficients and under predict wall temperatures. While this is a very rudimentary approach, it provides at least an upper bound for when dryout should occur. This method 
was used to ensure that the annulus is not too long and that the coolant will not reach a superheated state prior to exiting the annulus.

\section{IV.2.3 Post-Superheat Modeling}

Depending on the mass flux, applied heat flux and annulus length, among other parameters, dryout may or may not be reached. If it is reached, a post-dryout model is necessary to supplement the above model. In the homogeneous flow model, the point of dryout coincides with that of superheating. When enough heat has been absorbed by the fluid that it is entirely saturated vapor, the microscopic (i.e. phase change) effects become obsolete. That is, the total heat transfer is due only to the single phase convection of the superheated or saturated vapor.

Beyond this point, the single phase Rayleigh equations from Chapter II are used. The same iterative process described in the homogeneous flow model is applied with one modification. Because the quality of the flow is no longer defined, the density and thermal properties cannot be defined as a function of the quality. The density must be looked up from property tables based on pressure and enthalpy. Starting at the dryout location $z_{d o}$, the solution process is summarized briefly below.

The enthalpy is calculated as defined in equation (4.41):

$$
h_{z} \approx h_{z-d z}+\frac{d q}{\dot{m}}
$$


As a first approximation, the pressure at location $z$ is then assumed equal to the pressure at location $z-d z$ :

$$
P_{z} \approx P_{z-d z}
$$

A density and temperature are then looked up in tables based on the pressure and enthalpy given by equations (4.43) and (4.44):

$$
\begin{aligned}
& \rho_{z}=f\left(P_{z}, h_{z}\right) \\
& T_{z}=f\left(P_{z}, h_{z}\right)
\end{aligned}
$$

The pressure at location $z$ is then reevaluated from the Rayleigh line equation:

$$
P_{z}=P_{z-d z}+G^{2}\left(\frac{1}{\rho_{z-d z}}-\frac{1}{\rho_{z}}\right)
$$

The velocity at location $z$ is then given by continuity:

$$
u_{z}=\frac{G}{\rho_{z}}
$$

This result can be substituted back into the energy equation to account for changes in kinetic energy as was done in the homogeneous flow model prior to dryout. In the next iteration, the density is looked up as a function of the updated enthalpy and the pressure 
predicted by the Rayleigh line equation in the previous iteration. This process is repeated for each location $z$ until the change in pressure is less than 0.1 MPa. After the above iterative loop has finished for each location $z$, thermal properties are determined from tabulated data based on temperature and pressure:

$$
\begin{gathered}
\mu_{z}=f\left(T_{z}, P_{z}\right) \\
k_{z}=f\left(T_{z}, P_{z}\right) \\
C_{p_{z}}=f\left(T_{z}, P_{z}\right)
\end{gathered}
$$

Recall that these properties are defined for carbon dioxide rather than nitrous oxide due to a lack of data available for nitrous oxide as was discussed previously. The heat transfer coefficients are given by a single phase Nusselt number correlation:

$$
h_{\text {cold }_{z}}=0.023 \operatorname{Re}_{z}^{0.8} \operatorname{Pr}_{z}^{0.4} \frac{k_{z}}{D_{h}}
$$

where $R e_{z}$ and $\operatorname{Pr}_{z}$ are the Reynolds and Prandtl numbers of the gas at location $z$ respectively and are given by

$$
R e_{z}=\frac{\rho_{z} u_{z} D_{h}}{\mu_{z}}
$$

and 


$$
\operatorname{Pr}_{z}=\frac{\mu_{z} C_{p_{z}}}{k_{z}}
$$

The wall temperature in the post dryout region is again calculated from Newton's Law of Cooling:

$$
T_{\text {wall }_{z}}=\frac{q}{h_{\text {cold }_{z}} A_{\text {cold }}}+T_{z}
$$

One of the major limitations to the post-dryout model is that, due to its iterative nature, the density and thermal properties must be looked up manually. This limits the number of locations at which the properties can be calculated and, by extension, the grid size that can be used. However, the elevated temperatures accompanied by the inception of superheat make it desirable to avoid this regime of flow altogether. The most important aspect of the model with regards to the superheated vapor regime is that it be able to predict the conditions at which superheating occurs. It is unlikely that the model described in equations (4.41) through (4.53) should ever be used as if the physical system reached this point the nozzle would likely lose its structural integrity and be incapable of collecting data. The superheat model is included mostly for completeness not for practical use.

\section{IV.2.4 Preliminary Results}

The homogeneous flow model was run for Cal Poly hybrid rocket standard operating conditions ("SOC"). These are: (1) a line pressure of 800 psia, (2) a post-injector pressure 
of 350 psia, (3) a mass flow rate of $0.1 \mathrm{lbm} / \mathrm{sec}$, (4) an $\mathrm{O} / \mathrm{F}$ of 2.0 and (5) a chamber pressure of 200 psia. For these conditions, assuming a dryout quality of 1.0, the flow is not predicted to dryout. The quality is given as a function of distance through annulus in Figure 77.

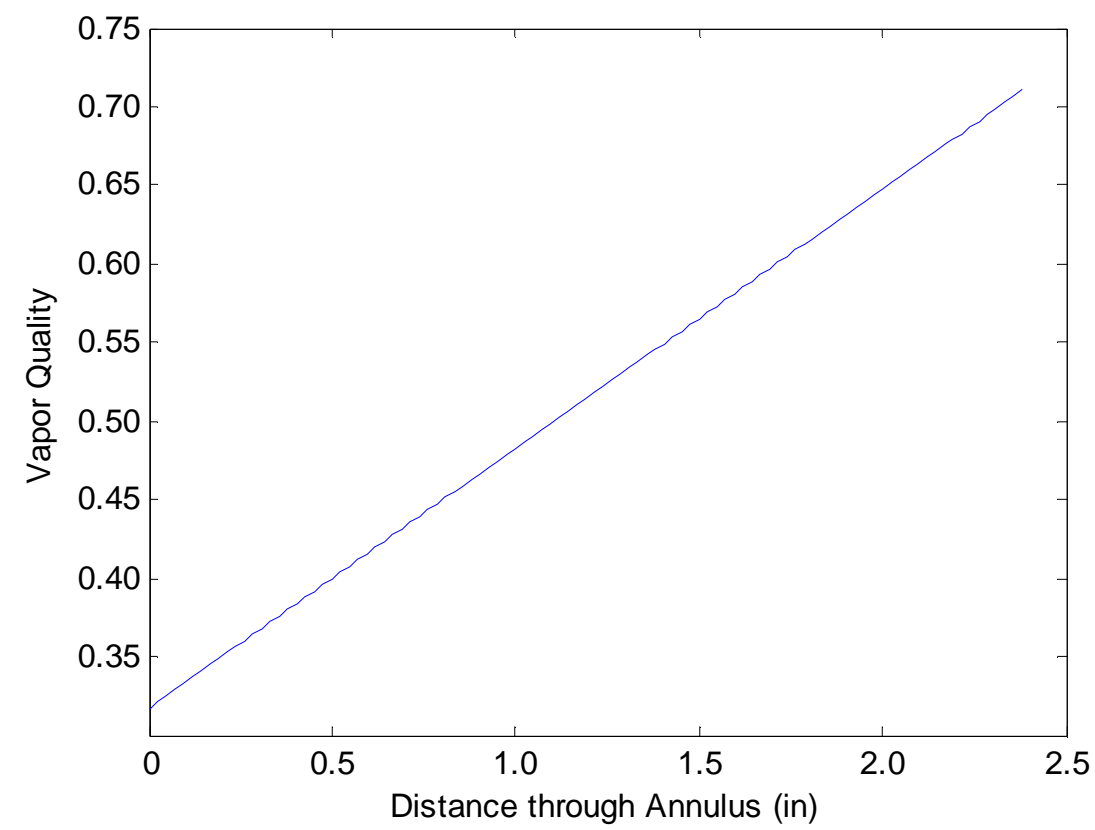

Figure 77. Vapor quality as predicted by the homogeneous flow model for SOC

The fluid temperature profile is shown in Figure 78. 


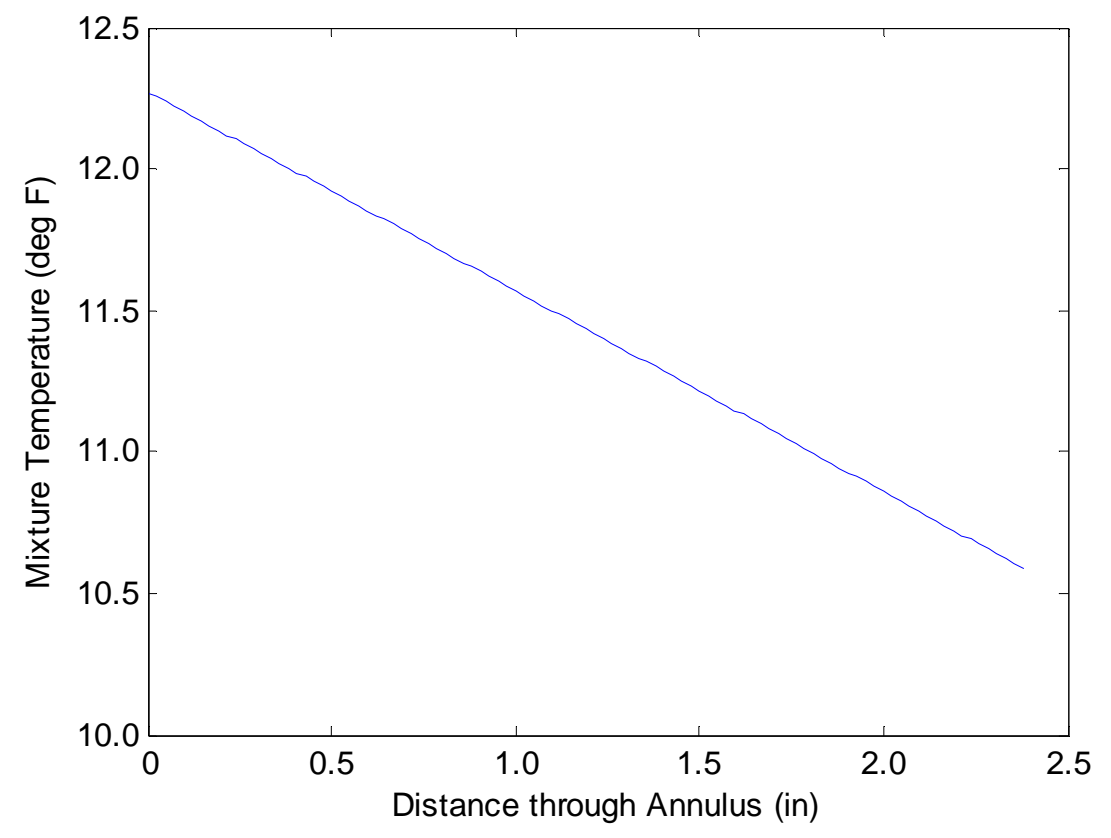

Figure 78. Fluid temperature as predicted by homogeneous flow model for SOC The copper well temperature is given in Figure 79.

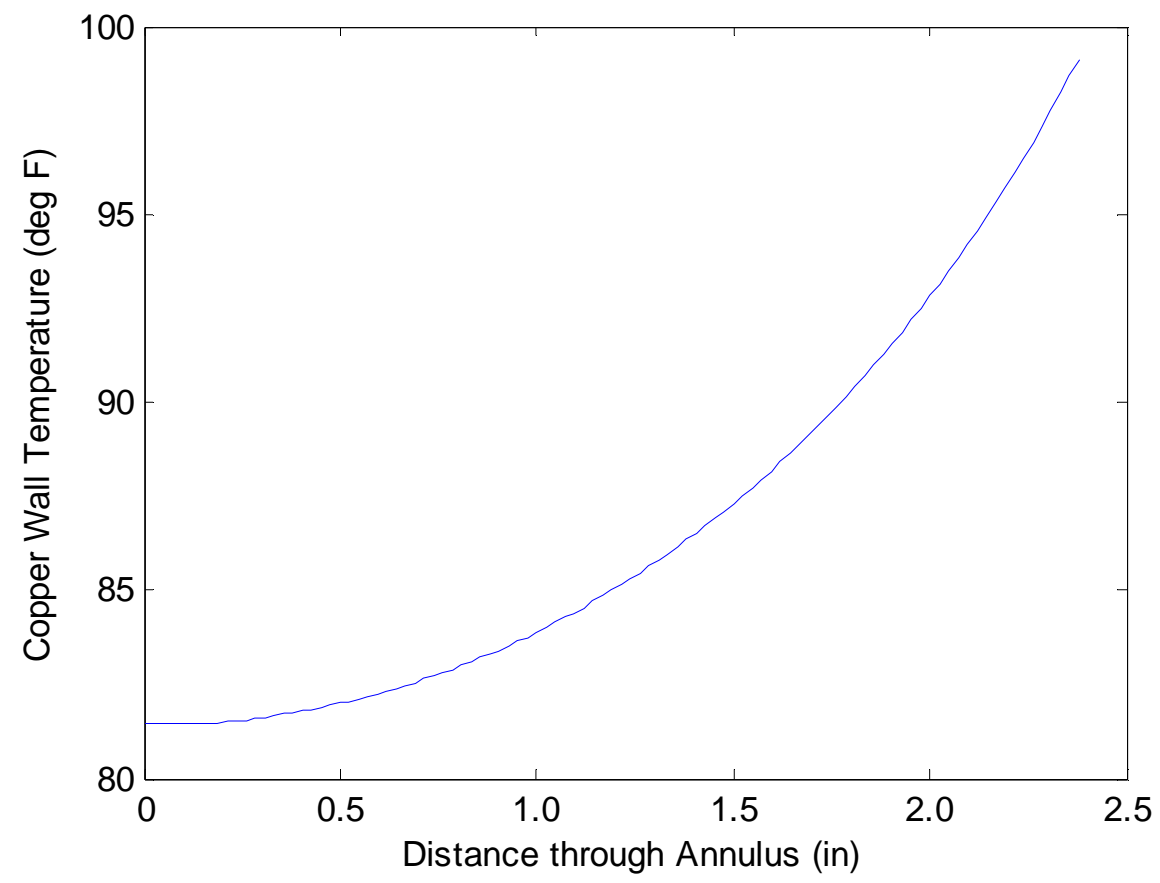

Figure 79. Copper wall temperature as predicted by homogeneous model for SOC 
The static fluid pressure profile is shown in Figure 80.

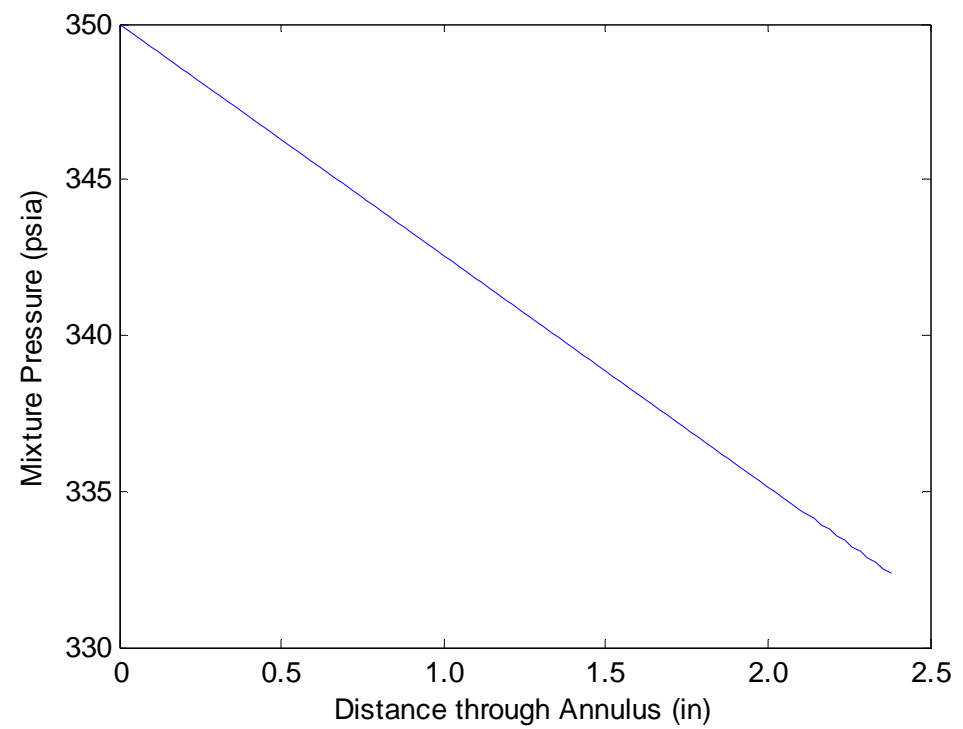

Figure 80. Fluid pressure as predicted by the homogeneous model for SOC

Finally, the heat transfer coefficient as predicted by the Shah correlation is shown in Figure 81 .

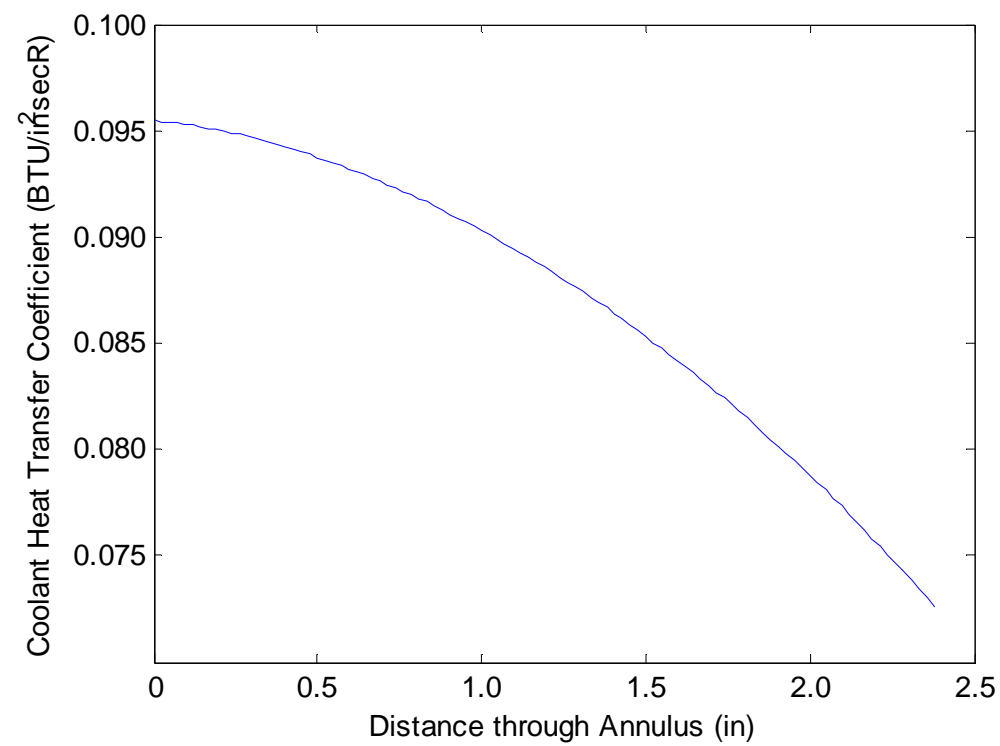

Figure 81. Predicted coolant heat transfer coefficient for SOC 
Notice that the heat transfer coefficients predicted by this model are much larger than (approximately 3 times) those seen in previous tests in Chapter II. Likewise, the copper temperatures are also much lower (refer to Figure 17). This suggests that the system may not be in the nucleate boiling regime throughout the entire annulus and may have reached dryout. A more advanced method is necessary for predicting dryout in order to determine whether or not this is the case. In order to predict this, some assumptions about the flow regime prior to dryout must be made. An annular flow regime will be assumed in order to proceed with the modeling process.

\section{IV.3 REVELLIN-THOME DRYOUT MODEL}

Revellin and Thome developed a separated phase with interface exchange model for annular flow in heated mini and microchannels with the intention of predicting dryout. This model is well-suited for the application at hand as the hydraulic diameter of the coolant annulus is approximately three millimeters which qualifies it as a "minichannel." The authors begin by developing a set of ordinary differential equations from generalized two-phase conservation laws. The control volume is shown in Figure 82 below: 


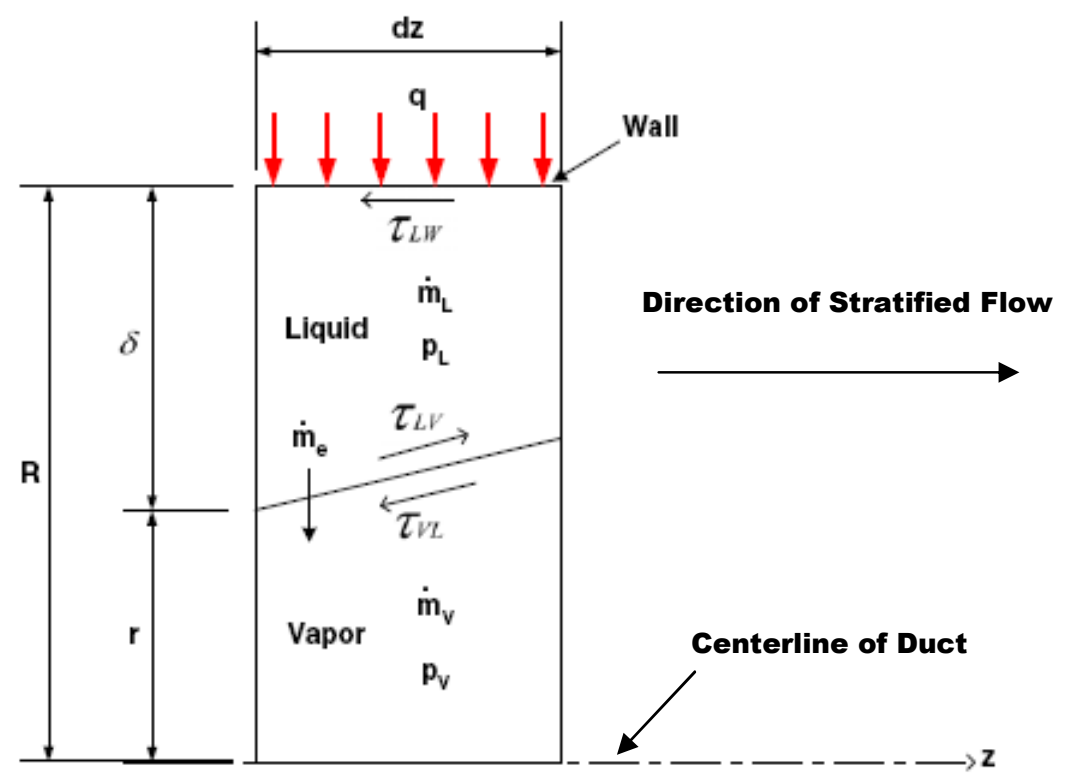

Figure 82. Annular separated flow model control volume(10)

Assuming the density and specific enthalpy of each phase remains constant at its saturation value, the set of ordinary differential equations (ODEs) governing the flow is given in equations (4.54) through (4.58) below:

$$
\begin{gathered}
\frac{d\left(A_{L} u_{L}\right)}{d z}=-\frac{q^{\prime \prime} P_{h}}{\left(h_{L}-h_{V}\right) \rho_{L}} \\
\frac{d\left(A_{V} u_{V}\right)}{d z}=\frac{q^{\prime \prime} P_{h}}{\left(h_{L}-h_{V}\right) \rho_{V}} \\
\rho_{L} \frac{d\left(A_{L} u_{L}^{2}\right)}{d z}=-A_{L} \frac{d P_{L}}{d z}+A_{i}\left|\tau_{L V}\right|-A_{L W}\left|\tau_{L W}\right| \\
\rho_{V} \frac{d\left(A_{V} u_{V}^{2}\right)}{d z}=-A_{V} \frac{d P_{V}}{d z}+A_{i}\left|\tau_{V L}\right| \\
\frac{d p_{V}}{d z}-\frac{d p_{L}}{d z}=\frac{d}{d z}\left(\frac{\sigma}{r}\right)
\end{gathered}
$$


where $A_{V}$ is the cross sectional area of annulus occupied by vapor and is given by

$$
A_{V}=\pi r^{2}
$$

and $r$ is the radius of the vapor cylinder at the center of the duct. Also note that $\delta$ is liquid film thickness and that the sum of $\delta$ and $r$ is the total radius of the cylinder $R$ :

$$
R=\delta+r
$$

$A_{L}$ is the cross sectional area of the annulus occupied by liquid and is given by

$$
A_{L}=\pi\left(R^{2}-r^{2}\right)
$$

The area of the liquid-vapor interface $A_{i}$ is equal to

$$
A_{i}=\sqrt{2} \pi(2 r+d z) d z
$$

$A_{L W}$ is the area of the liquid-wall interface and is given by

$$
A_{L W}=2 \pi R d z
$$

$\tau_{L W}$ is the shear stress between the liquid and the wall: 


$$
\tau_{L W}=\frac{1}{2} C_{f} \rho_{L} u_{L}^{2}
$$

where the friction factor $C_{f}$ is evaluated using the common correlation for turbulent flow:

$$
C_{f}=0.078 R e_{L}^{-0.25}
$$

and $R e_{L}$ is the liquid Reynolds number given by

$$
R e_{L}=\frac{2 \rho_{L} u_{L}(R-r)}{\mu_{L}}
$$

The liquid-vapor and vapor-liquid interfacial shear stresses $\left(\tau_{L V}\right.$ and $\left.\tau_{V L}\right)$ are equal and opposite:

$$
\tau_{L V}=-\tau_{V L}
$$

and are evaluated similarly to the liquid-wall interfacial shear stress:

$$
\tau_{L V}=\frac{1}{2} C_{f_{V}} \rho_{V} u_{V}^{2}
$$

In this case, because the flow is turbulent: 


$$
C_{f_{V}}=0.078 R e_{V}^{-0.25}
$$

where

$$
R e_{V}=\frac{2 \rho_{V} u_{V} r}{\mu_{V}}
$$

There are five unknowns in the previous set of equations: $r, u_{V}, u_{L}, P_{V}$, and $P_{L}$. Thome and Revellin assumed that a saturated liquid existed at the inlet to the duct which gives the following boundary conditions:

$$
\begin{gathered}
\left.r\right|_{z=0}=r_{\min } \\
\left.u_{V}\right|_{z=0}=\frac{G}{\rho_{L}} \\
\left.u_{L}\right|_{z=0}=\frac{G}{\rho_{L}} \\
\left.p_{V}\right|_{z=0}=p_{\text {sat }} \\
\left.p_{L}\right|_{z=0}=p_{\text {sat }}-\frac{\sigma}{r_{\text {min }}}
\end{gathered}
$$

The liquid film is assumed to dryout when the amplitude of the interfacial waves becomes greater than the liquid film thickness. This is summarized in Figure 83 below: 

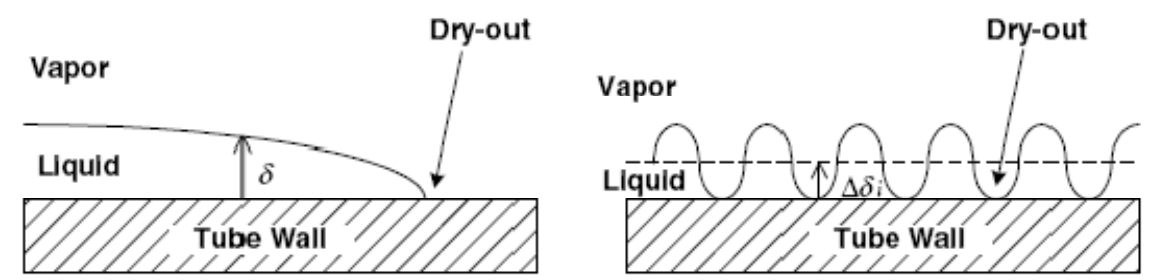

Figure 83. Interfacial wave dryout in annular flow regime

The amplitude of the interfacial waves is derived using the Kelvin-Helmholtz critical wavelength. Based on this, the flow is predicted to dryout when the liquid film thickness equals the critical film thickness value of

$$
\delta_{c}=C R\left(\frac{u_{V}}{u_{L}}\right)^{j_{1}}\left(\frac{\left(\rho_{L}-\rho_{V}\right) g R^{2}}{\sigma}\right)^{k_{1}}
$$

where $C, j_{1}$ and $k_{1}$ are empirically derived constants equal to $0.15,-3 / 7$ and $-1 / 7$ respectively, $\sigma$ is the surface tension and $g$ is the gravitational constant.

\section{IV.3.1 Method of Solution}

A numerical method was used to solve the above system of ODEs. The channel was first discretized as shown roughly in Figure 84 below.

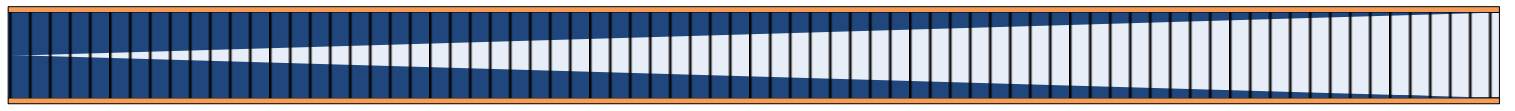

Figure 84. Discretized channel for numerical solution of separated flow ODEs 
A Taylor series method was then applied to solve for the five unknowns at each discretized section in the annulus. From the boundary conditions, the fluid at the inlet of the annulus is fully defined. Starting at the inlet, a "step through" method is used in which each location is solved for based on the previous location(s). Note that the order in which the equations are solved is crucial. Two separate solution passes are made through the annulus. First, a modified first order Taylor series method is used to develop an approximate solution. The accuracy of this pass is not critical. Next, the results of the first order Taylor series are used in conjunction with a third order Taylor series to provide a more accurate final solution. This method is described in more detail below.

Because the right hand side of equations (4.54) and (4.55) is a constant, they are integrated directly and solved to yield the product of the area and velocity of the liquid and vapor. The results are given by equations (4.77) and (4.78) below. Note that these are not approximations but exact solutions:

$$
\begin{aligned}
& \left(A_{L} u_{L}\right)_{n+1}=\left(A_{L} u_{L}\right)_{n}-\frac{q P}{h_{L V} \rho_{L}} \Delta \mathrm{z} \\
& \left(A_{V} u_{V}\right)_{n+1}=\left(A_{V} u_{V}\right)_{n}+\frac{q P}{h_{L V} \rho_{V}} \Delta \mathrm{z}
\end{aligned}
$$

Equation (4.56) is expanded using the product rule to yield

$$
A_{L} u_{L} \frac{d u_{L}}{d z}+u_{L} \frac{d\left(A_{L} u_{L}\right)}{d z}=\frac{1}{\rho_{L}}\left[-A_{L} \frac{d P_{L}}{d z}+\frac{A_{i}}{\Delta z}\left|\tau_{L V}\right|-\frac{A_{L W}}{\Delta z}\left|\tau_{L W}\right|\right]
$$


Substituting equation (4.54) in for the latter term on the left hand side of the equation gives

$$
A_{L} u_{L} \frac{d u_{L}}{d z}-u_{L} \frac{q^{\prime \prime} P_{h}}{\left(h_{L}-h_{V}\right) \rho_{L}}=\frac{1}{\rho_{L}}\left[-A_{L} \frac{d P_{L}}{d z}+\frac{A_{i}}{\Delta \mathrm{z}}\left|\tau_{L V}\right|-\frac{A_{L W}}{\Delta \mathrm{z}}\left|\tau_{L W}\right|\right]
$$

Solving for $u_{L}$ ' gives

$$
\frac{d u_{L}}{d z}=\frac{1}{A_{L} u_{L}}\left\{\frac{1}{\rho_{L}}\left[-A_{L} \frac{d P_{L}}{d z}+\frac{A_{i}}{\Delta \mathrm{z}}\left|\tau_{L V}\right|-\frac{A_{L W}}{\Delta \mathrm{z}}\left|\tau_{L W}\right|\right]+u_{L} \frac{q^{\prime \prime} P_{h}}{\left(h_{L}-h_{V}\right) \rho_{L}}\right\}
$$

This result is evaluated at the current annulus location (n) as follows:

$$
\begin{gathered}
\left.\frac{d u_{L}}{d z}\right|_{n}=\frac{1}{\left.A_{L} u_{L}\right|_{n}}\left\{\frac{1}{\rho_{L}}\left[-\left.A_{L_{n}} \frac{d P_{L}}{d z}\right|_{n}+\frac{A_{i_{n}}}{\Delta \mathrm{z}}\left|\tau_{L V}\right|_{n}-\frac{A_{L W}}{\Delta \mathrm{z}}\left|\tau_{L W}\right|_{n}\right]\right. \\
\left.+u_{L_{n}} \frac{q^{\prime \prime} P_{h}}{\left(h_{L}-h_{V}\right) \rho_{L}}\right\}
\end{gathered}
$$

The liquid velocity at the next location in the annulus $(n+1)$ can then be evaluated using a first order Taylor series:

$$
u_{L_{n+1}} \approx\left(u_{L}\right)_{n}+\left.w \frac{d u_{L}}{d z}\right|_{n} \Delta \mathrm{z}
$$


where $w$ is a relaxation factor that is necessary due to the non-linearity of equation (4.82). Note that the addition of this parameter makes the results of the first order Taylor series inaccurate. However, introducing this factor is critical because it allows the system of equations to be solved without diverging. The third order Taylor series will give a relatively accurate solution despite this relaxation factor. Based on this reasoning, the relaxation factor was allowed.

The cross sectional area of the duct occupied by liquid at location $n+1$ can then be determined by using the results of equations (4.77) and (4.78):

$$
A_{L_{n+1}}=\frac{\left(A_{L} u_{L}\right)_{n+1}}{\left(u_{L}\right)_{n+1}}
$$

The radius of the vapor phase at location $n+1$ can then be determined by rearranging equation (4.61) and using the results of the result of equation (4.84) above:

$$
r_{n+1}=\sqrt{-\frac{\left(A_{L}\right)_{n+1}}{\pi}+R^{2}}
$$

The area of the annulus occupied by vapor at location $n+1$ can then be determined:

$$
A_{V_{n+1}}=\pi r_{n+1}^{2}
$$


Next, the velocity of the vapor at step $n+1$ can be found using the results of equations (4.78) and (4.86):

$$
u_{V n+1}=\frac{\left(A_{V} u_{V}\right)_{n+1}}{\left(A_{V}\right)_{n+1}}
$$

The interfacial area is a function of $r$ only and is calculated by

$$
A_{i_{n+1}}=\sqrt{2} \pi\left(2 r_{n+1}+d z\right) d z
$$

Equation (4.57) can be rearranged to give the derivative of vapor pressure at location $\mathrm{n}$ :

$$
\left.\frac{d P_{V}}{d z}\right|_{n}=\frac{1}{A_{V_{n}}}\left[A_{i_{n}}\left|\tau_{V L}\right|_{n}-\left.w \rho_{V} \frac{d\left(A_{V} u_{V}^{2}\right)}{d z}\right|_{n}\right]
$$

where the relaxation parameter is again used to allow convergence and

$$
\left.\frac{d\left(A_{V} u_{V}^{2}\right)}{d z}\right|_{n} \approx \frac{A_{V_{n+1}} u_{V_{n+1}}{ }^{2}-A_{V_{n}} u_{V_{n}}{ }^{2}}{\Delta \mathrm{z}}
$$

Then, the derivative at liquid pressure at location $n+1$ is approximated by equation (4.91) below: 


$$
\left.\left.\frac{d p_{L}}{d z}\right|_{n+1} \approx \frac{d p_{V}}{d z}\right|_{n}-\left.w \frac{d}{d z}\left(\frac{\sigma}{r}\right)\right|_{n+\frac{1}{2}}
$$

where the relaxation factor is again necessary to prevent divergence. The second term on the right hand side of equation (4.91) is evaluated using a first order Taylor series given by

$$
\frac{d}{d z}\left(\frac{\sigma}{r}\right) \approx \frac{\sigma}{\Delta \mathrm{z}}\left[\frac{1}{r_{n+1}}-\frac{1}{r_{n}}\right]
$$

Note that ideally the vapor pressure gradient at location $n+1$ would be used in equation (4.91) rather than that at location n. However, this value is not yet known and therefore cannot be used. Similarly, equation (4.92) must to be evaluated at $n+1 / 2$ rather than $n+1$ as not enough information is available at this point. This was deemed acceptable as this solution is simply meant to be a first order approximation and a more accurate solution will be found with the third order Taylor series. The void fraction at location $n+1$ is then given by

$$
\alpha_{n+1}=\frac{A_{v_{n+1}}}{A}=\frac{r_{n+1}^{2}}{R^{2}}
$$

The quality at location $n+1$ is calculated by rearranging equation (3.6): 


$$
x_{n+1}=\frac{\rho_{v} u_{v_{n+1}} \alpha_{n+1}}{G}
$$

Next, the liquid and vapor pressures at location $n+1$ can be derived using the results of equations (4.89) and (4.91) above in a first order Taylor series approximation:

$$
\begin{aligned}
& P_{V_{n+1}} \approx P_{V_{n+1}}+\left.\frac{d P_{V}}{d z}\right|_{n} \Delta \mathrm{z} \\
& P_{L_{n+1}} \approx P_{L_{n+1}}+\left.\frac{d P_{L}}{d z}\right|_{n} \Delta \mathrm{z}
\end{aligned}
$$

The liquid and vapor Reynolds numbers at location $n+1$ are evaluated using equations (4.66) and (4.70) respectively:

$$
\begin{gathered}
R e_{L_{n+1}}=\frac{2 \rho_{L} u_{L_{n+1}}\left(R-r_{n+1}\right)}{\mu_{L}} \\
\operatorname{Re}_{V_{n+1}}=\frac{2 \rho_{V} u_{V+1} r_{n+1}}{\mu_{V}}
\end{gathered}
$$

Using these results, the friction factors for the liquid-wall and liquid-vapor interfaces are evaluated at location $n+1$ : 


$$
\begin{gathered}
C_{f_{n+1}}=0.078 R e_{L_{n+1}}-0.25 \\
C_{f_{V_{n+1}}}=0.078 R e_{V_{n+1}}^{-0.25}
\end{gathered}
$$

Finally, the liquid-wall and liquid-vapor shear stresses are evaluated at location $n+1$ using the above results:

$$
\begin{gathered}
\tau_{L W_{n+1}}=\frac{1}{2} C_{f_{n+1}} \rho_{L} u_{L_{n+1}}{ }^{2} \\
\tau_{L V_{n+1}}=\frac{1}{2} C_{f_{V_{n+1}}} \rho_{V} u_{V_{n+1}}{ }^{2}
\end{gathered}
$$

The thickness of the liquid film is evaluated at $n+1$ by

$$
\delta_{n+1}=R-r_{n+1}
$$

At each step, the liquid film thickness is checked to ensure that it is not greater than the critical liquid film thickness given by

$$
\delta_{c_{n+1}}=C R\left(\frac{u_{V_{n+1}}}{u_{L_{n+1}}}\right)^{j_{1}}\left(\frac{\left(\rho_{L}-\rho_{V}\right) g R^{2}}{\sigma}\right)^{k_{1}}
$$

If $\delta>\delta_{\mathrm{c}}$, the flow has reached dryout and the model is no longer applicable. The algorithm described in equations (4.77) through (4.104) is performed for each section of the discretized annulus. Because additional accuracy is desirable, a second pass is then 
made through the annulus using a third order Taylor series approximation. The algorithm is very similar to that described above. However, equations (4.83), (4.95) and (4.96) are replaced by the more accurate third order Taylor series approximations below:

$$
\begin{aligned}
& u_{L_{n+1}} \approx u_{L_{n}}+\left.\frac{d u_{L}}{d z}\right|_{n} \Delta \mathrm{z}+\left.\frac{1}{2} \frac{d^{2} u_{L}}{d z^{2}}\right|_{n} \Delta \mathrm{z}^{2}+\left.\frac{1}{6} \frac{d^{3} u_{L}}{d z^{3}}\right|_{n} \Delta \mathrm{z}^{3} \\
& P_{V_{n+1}} \approx P_{V_{n+1}}+\left.\frac{d P_{V}}{d z}\right|_{n} \Delta \mathrm{z}+\left.\frac{1}{2} \frac{d^{2} P_{V}}{d z^{2}}\right|_{n} \Delta \mathrm{z}^{2}+\left.\frac{1}{6} \frac{d^{3} P_{V}}{d z^{3}}\right|_{n} \Delta \mathrm{z}^{3} \\
& P_{L_{n+1}} \approx P_{L_{n+1}}+\left.\frac{d P_{L}}{d z}\right|_{n} \Delta \mathrm{z}+\left.\frac{1}{2} \frac{d^{2} P_{L}}{d z^{2}}\right|_{n} \Delta \mathrm{z}^{2}+\left.\frac{1}{6} \frac{d^{3} P_{L}}{d z^{3}}\right|_{n} \Delta \mathrm{z}^{3}
\end{aligned}
$$

where the second and third order velocity derivatives are approximated from the first order Taylor series solution:

$$
\begin{aligned}
& \left.\frac{d^{2} u_{L}}{d z^{2}}\right|_{n} \approx \frac{\left.\frac{d u_{L}}{d z}\right|_{n+1}-\left.\frac{d u_{L}}{d z}\right|_{n-1}}{\Delta \mathrm{z}} \\
& \left.\frac{d^{3} u_{L}}{d z^{3}}\right|_{n} \approx \frac{\left.\frac{d^{2} u_{L}}{d z^{2}}\right|_{n+1}-\left.\frac{d^{2} u_{L}}{d z^{2}}\right|_{n-1}}{\Delta \mathrm{z}}
\end{aligned}
$$

Similarly, the liquid and vapor pressure differentials are approximated from the first order Taylor series results: 


$$
\begin{aligned}
& \left.\frac{d^{2} P_{L}}{d z^{2}}\right|_{n} \approx \frac{\left.\frac{d P_{L}}{d z}\right|_{n+1}-\left.\frac{d P_{L}}{d z}\right|_{n-1}}{\Delta \mathrm{z}} \\
& \left.\frac{d^{3} P_{L}}{d z^{3}}\right|_{n} \approx \frac{\left.\frac{d^{2} P_{L}}{d z^{2}}\right|_{n+1}-\left.\frac{d^{2} P_{L}}{d z^{2}}\right|_{n-1}}{\Delta \mathrm{z}} \\
& \left.\frac{d^{2} P_{V}}{d z^{2}}\right|_{n} \approx \frac{\left.\frac{d P_{V}}{d z}\right|_{n+1}-\left.\frac{d P_{V}}{d z}\right|_{n-1}}{\Delta \mathrm{z}} \\
& \left.\frac{d^{3} P_{V}}{d z^{3}}\right|_{n} \approx \frac{\left.\frac{d^{2} P_{V}}{d z^{2}}\right|_{n+1}-\left.\frac{d^{2} P_{V}}{d z^{2}}\right|_{n-1}}{\Delta \mathrm{z}}
\end{aligned}
$$

Also, in the third order solution, equations (4.90), (4.91) and (4.92) are modified to give more accurate first order Taylor series approximations by using more accurate indexing as shown below:

$$
\begin{gathered}
\left.\left.\frac{d p_{L}}{d z}\right|_{n} \approx \frac{d p_{V}}{d z}\right|_{n}-\left.\frac{d}{d z}\left(\frac{\sigma}{r}\right)\right|_{n} \\
\left.\frac{d}{d z}\left(\frac{\sigma}{r}\right)\right|_{n} \approx \frac{\sigma}{\Delta \mathrm{z}}\left[\frac{1}{r_{n+1}}-\frac{1}{r_{n-1}}\right] \\
\left.\frac{d\left(A_{V} u_{V}^{2}\right)}{d z}\right|_{n} \approx \frac{A_{V_{n+1}} u_{V_{n+1}}{ }^{2}-A_{V_{n-1}} u_{V_{n-1}}{ }^{2}}{\Delta \mathrm{z}}
\end{gathered}
$$

Also note that all relaxation parameters are excluded from the third order Taylor series approximation. In the second pass through with the modified equations, the dryout criterion is again evaluated at each step. If the liquid film thickness exceeds the critical liquid film thickness (equal to the amplitude of the interfacial waves), dryout has 
occurred and the model is no longer valid. Note that while mostly third order Taylor series approximations are used in the second solution, its inputs depend on the first order Taylor series approximation. The formal accuracy of this solution is therefore limited to first order. While this is not ideal, it is accepted as developing a model of this complexity requires compromises in accuracy. Also, the priority of this model is to give a binary answer as to whether or not dryout occurs in the annulus. With such an application, the order of the accuracy is not critical.

\section{IV.3.2 Solution Validation}

As is the case with any numerical solution, proper validation is necessary. In this case, a solution to this model was presented by Thome and Revellin for R-134a at a mass flux $G$ of $500 \mathrm{~kg} / \mathrm{m}^{2} \mathrm{~s}$, a saturation temperature of $30^{\circ} \mathrm{C}$ and an applied heat flux of $123 \mathrm{~kW} / \mathrm{m}^{2}$. The annulus for which this solution was presented had a diameter of $0.5 \mathrm{~mm}$ and a length of $70 \mathrm{~mm}$. The solution predicted dryout at the end of the annulus. Prior to applying this model to the nitrous oxide system of interest, it was necessary to confirm that it was implemented correctly by comparing the results to those presented for R-134a.

For R-134a, the saturation pressure corresponding to a temperature of $30^{\circ} \mathrm{C}$ is $7.7 \mathrm{MPa}$. At these conditions, the saturated liquid and vapor densities are 1187.5 and $37.5 \mathrm{~kg} / \mathrm{m}^{3}$ respectively. Likewise, the saturated liquid and vapor enthalpies are 214.72 and 414.82 $\mathrm{kJ} / \mathrm{kg}$. At $30^{\circ} \mathrm{C}$, the liquid and vapor dynamic viscosities are $183 \mathrm{E}-6$ and $11.9 \mathrm{E}-6$ $\mathrm{kg} /((\mathrm{m})(\mathrm{sec}))$ respectively. Finally, the surface tension is $0.007417 \mathrm{~N} / \mathrm{m}(19)$. 
The liquid film profile as a function of distance through annulus is shown in Figure 85 and Figure 86 below. The predicted liquid film thickness at the outlet of the channel for the Revellin and Thome model is 17.4 micrometers. That predicted using the third order Taylor series algorithm discussed in the previous section is 15.2 micrometers. The absolute error between these two values is 2.4 micrometers.

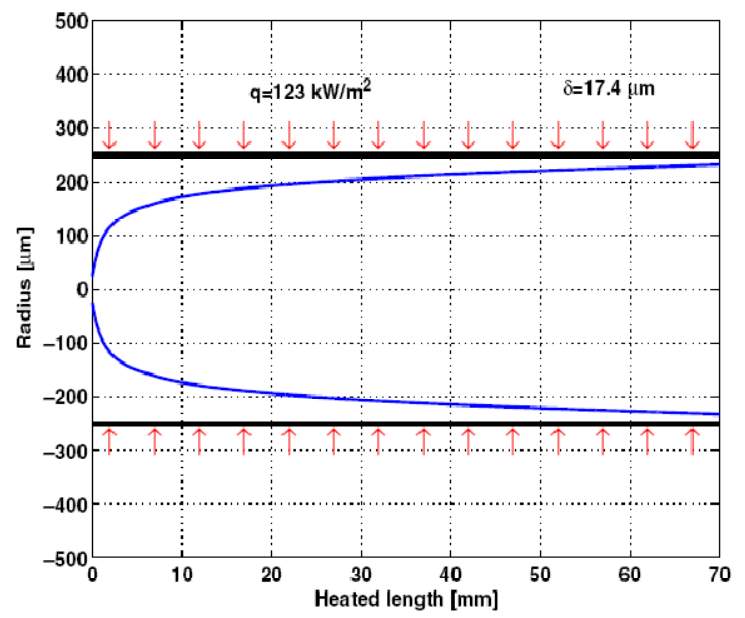

Figure 85. Liquid film profile predicted Revellin and Thome (10)

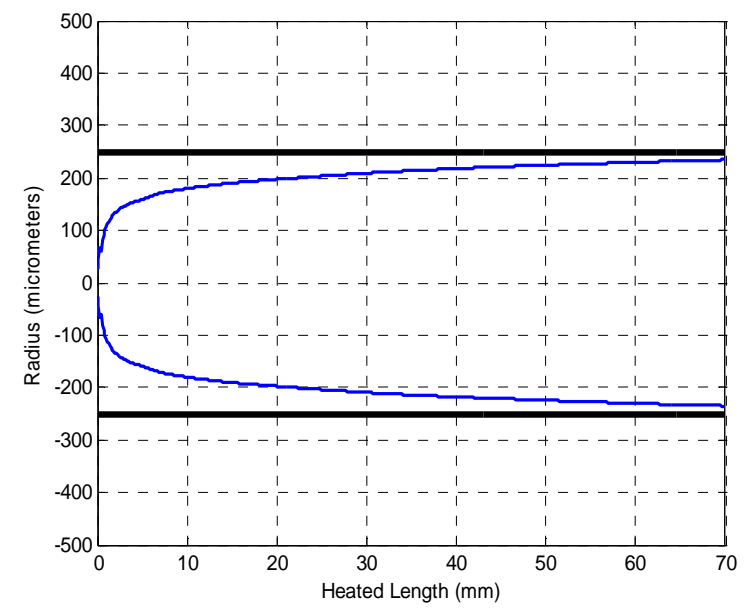

Figure 86. Liquid film profile predicted by Taylor series approximation 
The velocity profiles of the liquid and vapor phases of R-134 predicted by both models are shown in Figure 87 and Figure 88 below. Notice that there is some instability in the Taylor series solution toward the beginning of the annulus. This is likely due to the fact that the third order solution is incapable of solving for the first three locations in the annulus. The first order approximation must be relied on for these locations.

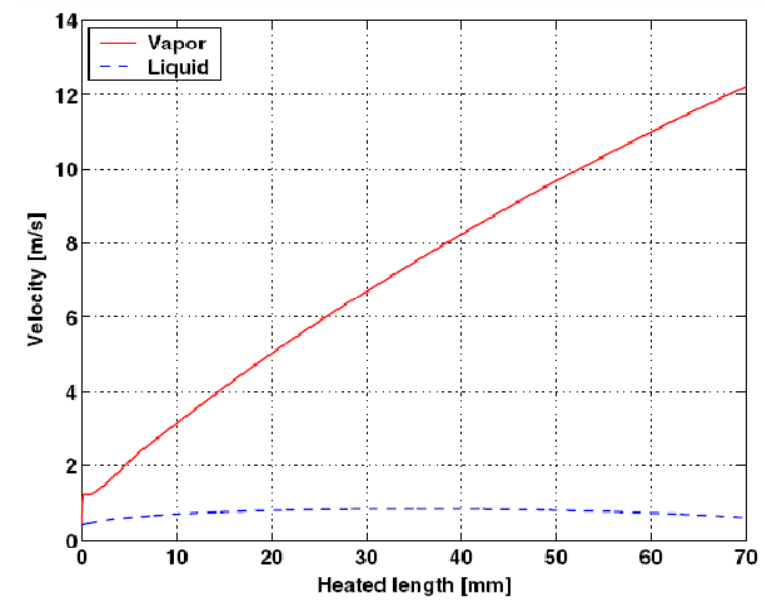

Figure 87. Liquid and vapor velocities predicted by Revellin and Thome (10)

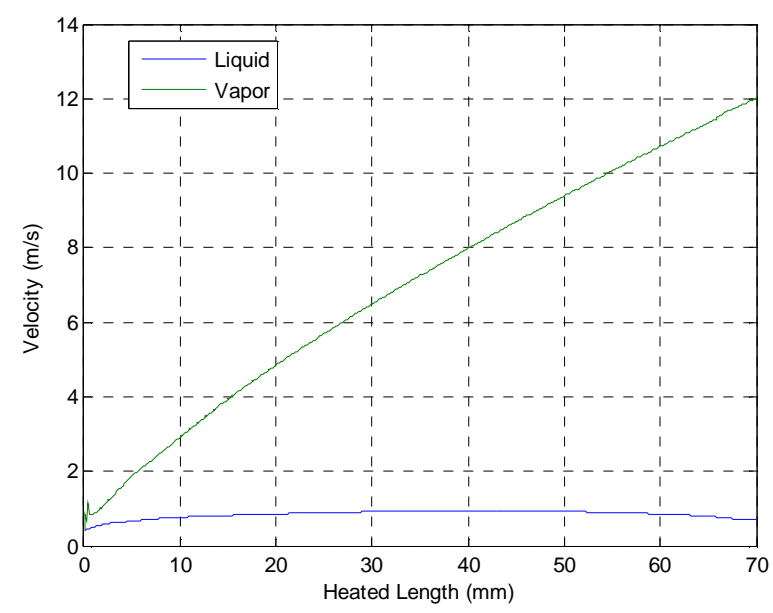

Figure 88. Liquid and vapor velocities predicted by Taylor series approximation 
Finally, the liquid and vapor pressures as a function of distance through the annulus are shown in Figure 89 and Figure 90 below. The pressure drop predicted by Revellin and Thome is 1.94 bar per meter. The third order Taylor series method predicts a pressure drop of 2.65 bar per meter.

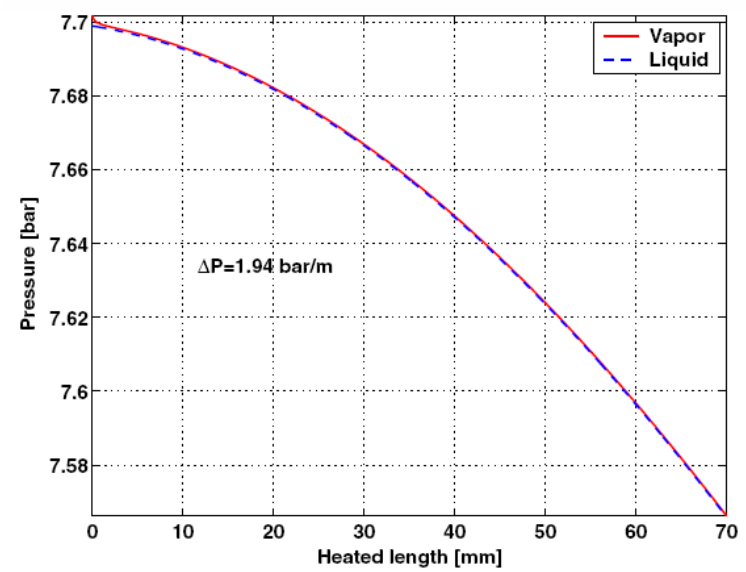

Figure 89. Liquid and vapor pressures predicted by Revellin and Thome (10)

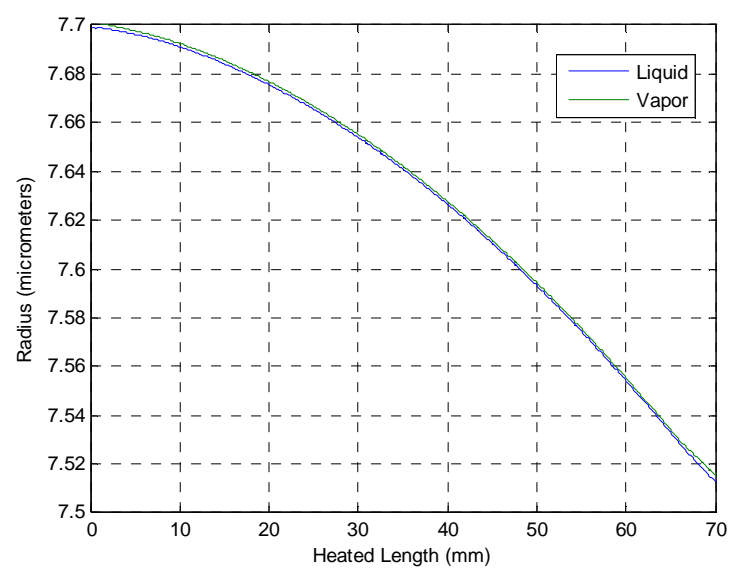

Figure 90. Liquid and vapor pressures predicted by Taylor series approximation 
While there are some significant discrepancies between the results given by solution method presented in previous sections and those from Thome and Revellin, the general predicted trends are the same.

\section{IV.3.3 Application to Nitrous Oxide}

The model in the previous section was developed and validated for a saturated inlet boundary condition. This does not apply to the nitrous oxide cooling system as the injector upstream of the annulus throttles the system from a saturated liquid to a quality of approximately twenty to thirty percent. Based on this, considerable modifications are necessary to allow the Revellin-Thome model to be applied to the nitrous oxide system.

The first and most obvious method of adapting the model is to simply change the boundary conditions such that the velocities, pressures and, by extension, vapor radius are equal to those at the actual inlet quality. However, the saturated inlet boundary condition allows the assumption that the velocities of both phases are equal to that of the liquid phase. By this logic, removing this boundary condition introduces another variable which cannot be easily accounted for. Also, the difficulties encountered with reaching a stable solution in the previous section suggest that changing any boundary conditions may result in divergence. A decision was therefore made to allow the saturated inlet boundary conditions to remain. Instead, an extra length of pipe is added to the nitrous oxide model in which the mixture is heated from saturated liquid $(x=0)$ to the postinjector quality as given by equation (4.2) $(x \approx 0.2)$. In this way, the injector in the 
physical system is modeled as a heated length of pipe. This is depicted in Figure 91 below:
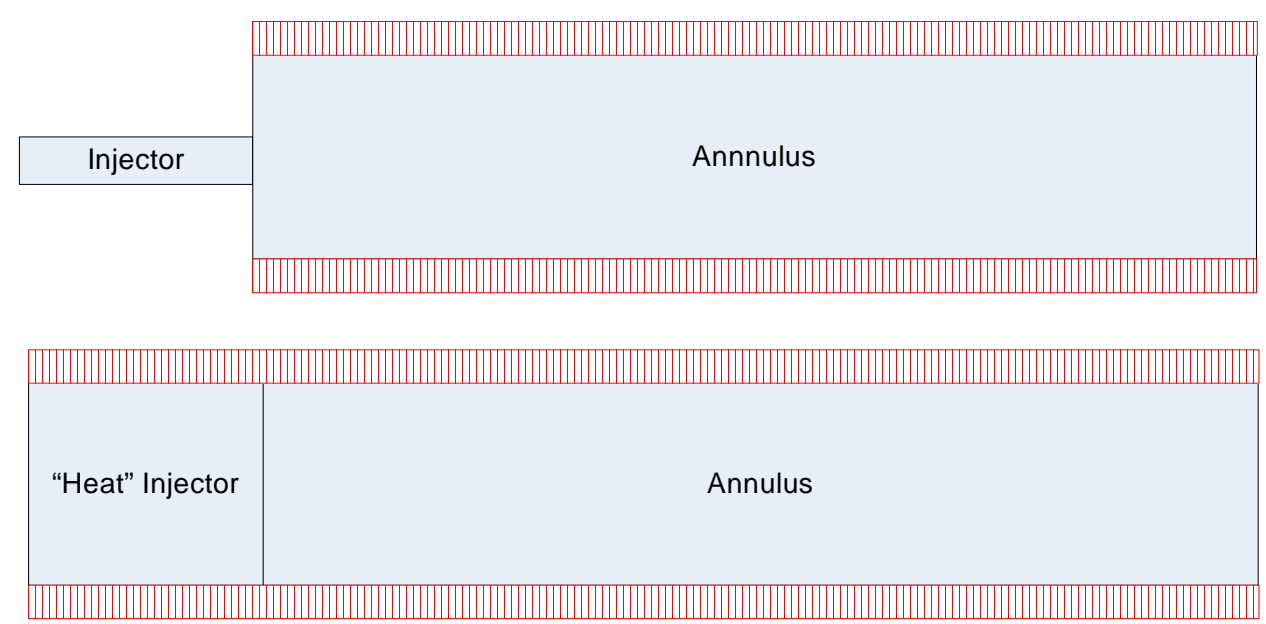

Figure 91. Physical two-phase injector (top) and two-phase injector model (bottom)

Finally, the relaxation parameter was no longer necessary to prevent divergence in this run and was therefore removed from the solution. The grid spacing used for the validated R-134a model is again used in this model. Note that one major limitation of the heated injector model is that it does not guarantee that the liquid and vapor speeds match at the inlet the annulus. However, it allows the remainder of the model to be run and is therefore used. It should be noted that there may be a significant amount of error introduced by this injector model.

\section{IV.3.4 Results}

The model was run for typical Cal Poly hybrid rocket operating conditions. These are a line pressure of 800 psia, a post-injector pressure of 350 psia, a mass flow rate of 0.1 
$\mathrm{lbm} / \mathrm{sec}$, an $\mathrm{O} / \mathrm{F}$ of 2.0 and a chamber pressure of $200 \mathrm{psia}$. For these conditions, the flow is predicted to dryout five millimeters into the sixty millimeter long annulus at a quality of $38 \%$. The static fluid pressure profile is shown in Figure 92:

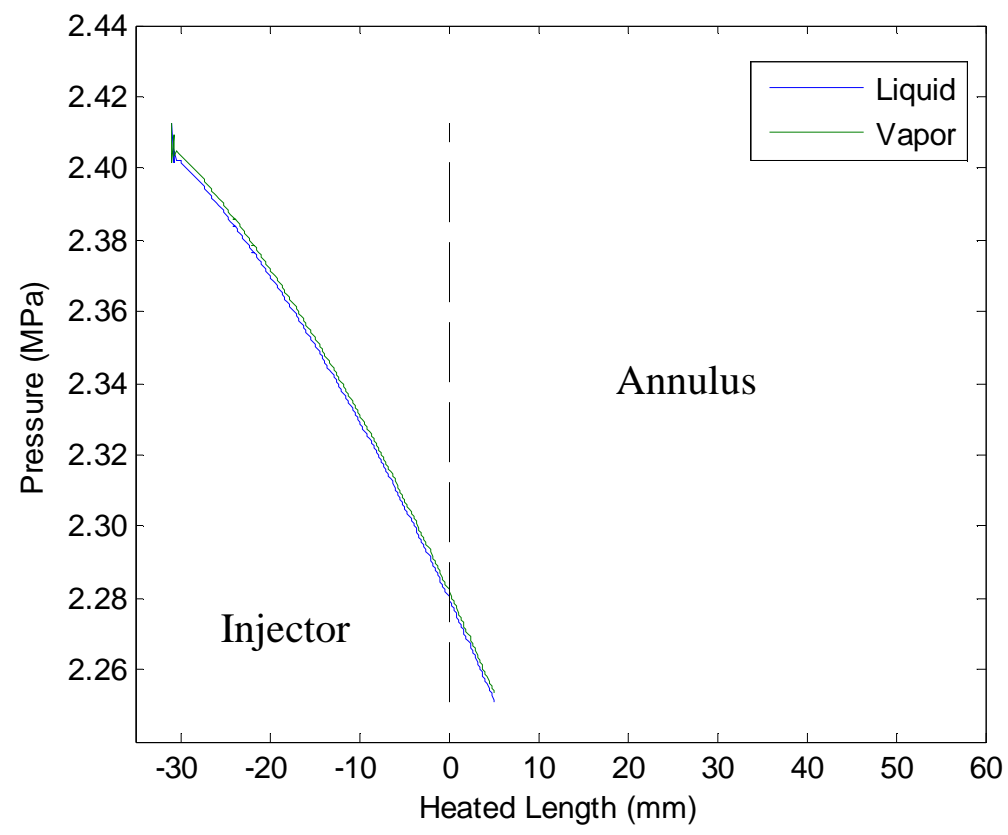

Figure 92. Predicted Static fluid pressures through injector and annulus until dryout

The quality as a function of distance through the annulus is shown in Figure 93: 


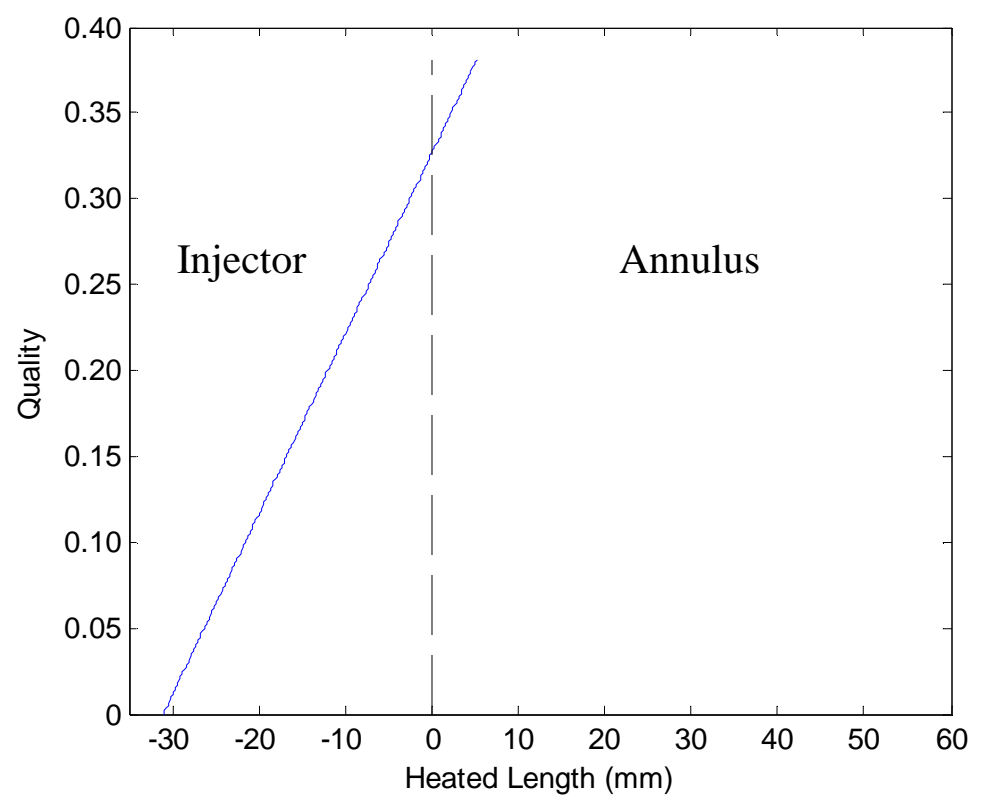

Figure 93. Predicted quality through injector and annulus until dryout

The relatively low dryout quality suggests that mist flow exists in much of the annulus rather than the assumed annular flow. This result makes sense with the data collected in previous tests as the heat transfer coefficients predicted by the Shah correlation (for the nucleate boiling regime) are much higher than those seen in the physical system. It is also unlikely that inverted annular flow exists as the copper wall temperatures seen in previous tests were relatively mild for the applied heat flux. Mist flow heat transfer coefficients are higher than those seen in inverted annular flow and lower than those seen in nucleate boiling. Based on this logic, it is very possible that the nitrous oxide within the cooling system annulus is in the mist flow regime. Whatever the case, the RevellinThome model is not applicable for modeling the hydrodynamic characteristics in the majority of the annulus. This model does provide invaluable information in that it predicts the location in the annulus and the quality at which dryout will occur. 
Also note that the heated injector model may not be entirely accurate. In fact, it seems that the physical injector may act as an atomizer, inducing mist flow at the very inlet of the annulus such that annular flow never exists. Novak studied this concept with multiphase air-water mixtures in a straight duct with an unheated entrance length over a wide range of operating conditions (23). These tests showed that even the slightest variation in operating conditions would have a significant effect on heat transfer coefficient. Such parameters are the: (1) geometry of the injector used to create these flows, (2) applied heat load, (3) carrier gas velocity, (4) carrier gas temperature, and (5) tube diameter, among many other parameters. Each of these conditions has a direct affect on the size of the liquid droplets entrained in the vapor bulk and by extension the heat transfer coefficients. An image of mist flow is shown in Figure 94.

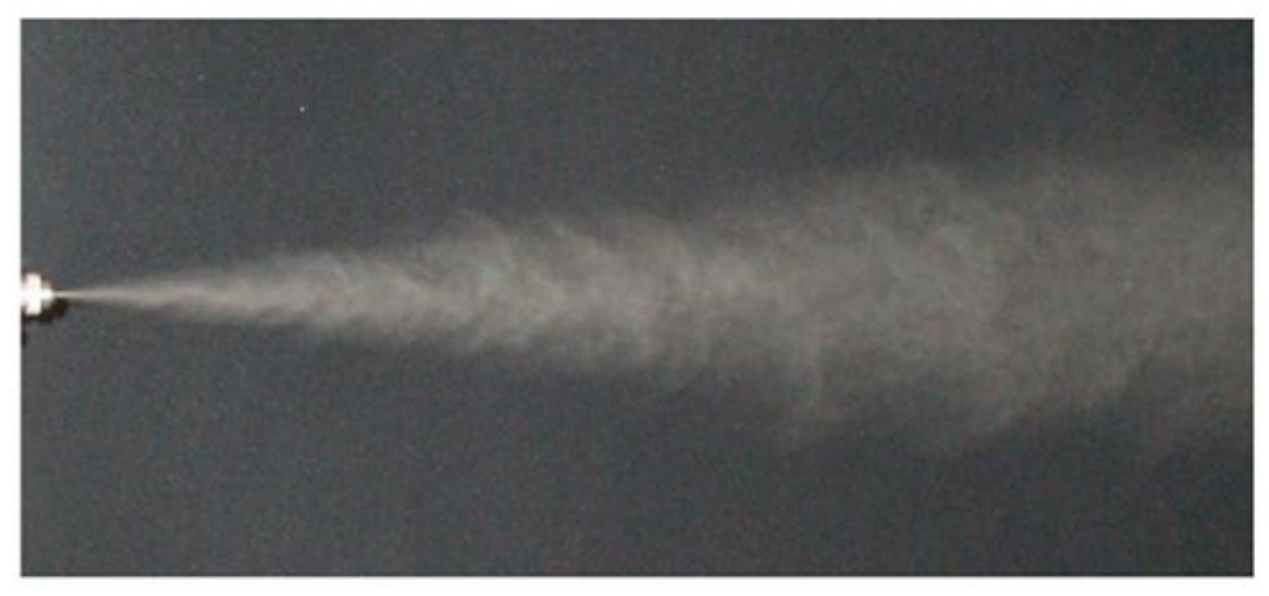

Figure 94. Mist flow (in contrast to previously discussed flow regimes)(23)

Based on the relatively short annular flow region predicted by the Revellin-Thome model and the likelihood that the inlet injector provokes a mist flow, for the operating conditions for which the Revellin-Thome model was run above, the annular entrance 
region will be neglected. Mist flow will be assumed throughout the entire annulus. However, it is still important to maintain the Revellin-Thome algorithm in the general two-phase model as it may become important with lower mass and heat fluxes. In the general model, if the predicted dryout location occurs less than $25 \%$ into the annulus, the annular flow regime will be neglected.

\section{IV.4 FINAL TWO-PHASE MODELING}

Based on the results of both the homogeneous and Revellin-Thome models, a combination of the two will be used as the final nitrous oxide system model. The Revellin-Thome model suggests that mist flow rather than the assumed annular flow occurs in the majority of the annulus. Carey outlines a number of different correlations for modeling mist flows (5) the first of which is the homogeneous flow model which has already been formulated in the previous section and is therefore especially convenient. Unfortunately, the Shah correlation is only applicable to pre-CHF (pre-dryout) flows. The Revellin-Thome model suggests that dryout in the system of interest occurs at qualities far lower than unity (approximately 0.4). Based on this, a new method is necessary for formulating heat transfer coefficients between the dryout quality and the saturated vapor line. Unfortunately no empirical correlations exist that are applicable to nitrous oxide in the mist flow regime. The Groeneveld correlation is one of the few heat transfer coefficient correlations developed for mist flow but is applicable to steam-water mixtures only. Ideally, enough data would be collected to produce a correlation similar to that of Groeneveld for the system of interest. However, this is not the case and therefore no method of predicting heat transfer coefficients is offered at this time. 
In the case that the vapor becomes superheated, the post-dryout model developed in accordance with the homogeneous flow model in which the Rayleigh equations and single-phase heat transfer coefficients were developed will be used. This is summarized in Figure 95 on the following page. Note that as was discussed previously, if the Revellin-Thome model predicts dryout at a location less than $25 \%$ into the annulus, mist flow will be assumed throughout the entire annulus.

Note that not all of the models must be used for any given scenario. The algorithm simply runs until the end of the annulus is reached. For example, if the applied heat flux is sufficiently below the CHF throughout the entire annulus, the flow will never reach dryout and will therefore be modeled by the Revellin-Thome and Shah models during its entire time in the annulus. Likewise, if the critical heat flux is exceeded but not enough heat is added to the flow in the mist flow regime to superheat it, the superheat portion of the model will not be used. All models listed on the flowchart are described in previous sections with the exception of the Groeneveld correlation. For the sake of brevity, these models will therefore not be reiterated. 

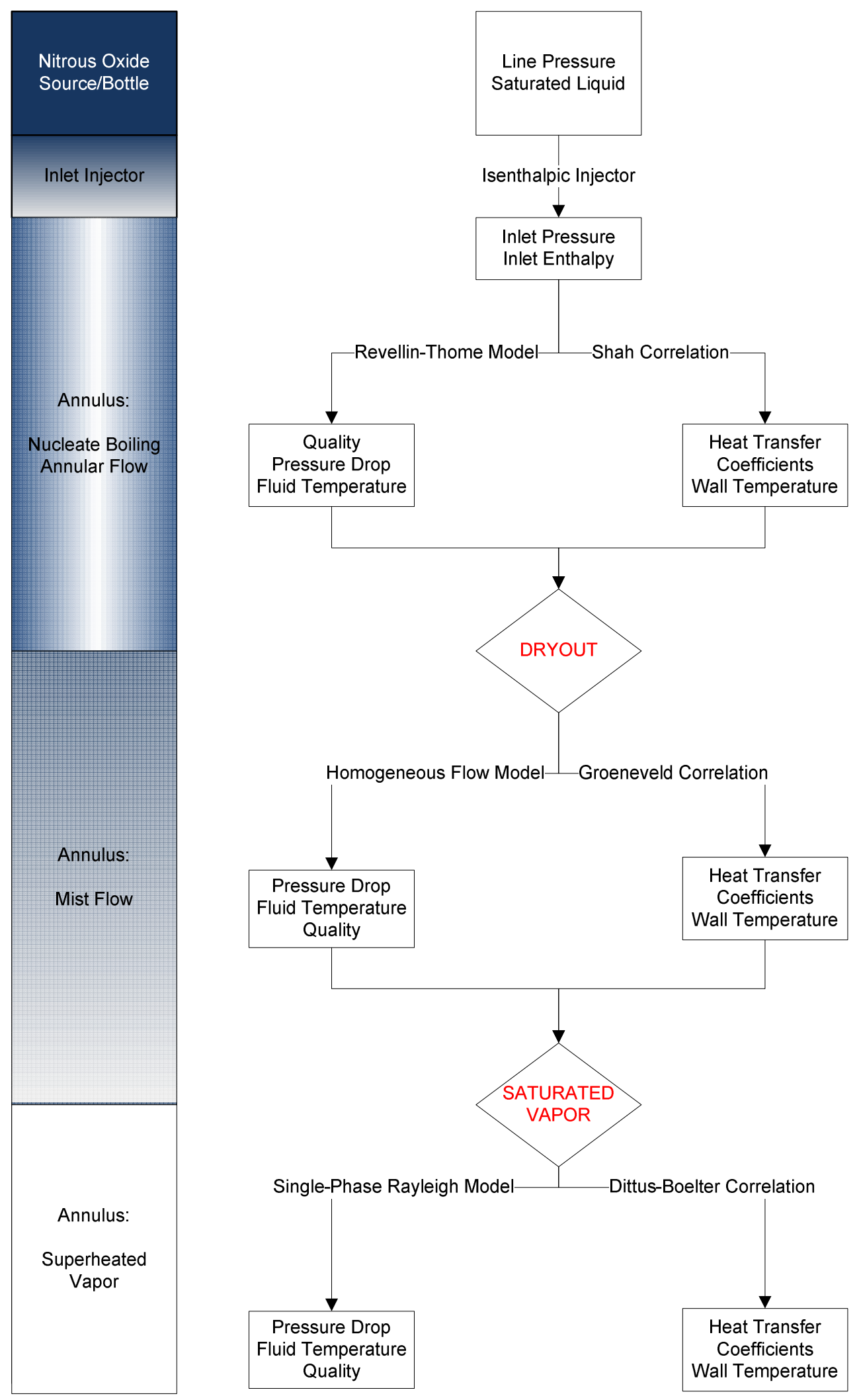

Figure 95. Final two-phase model flow chart 


\section{IV.4.1 Groeneveld Correlation}

The mist flow regime is notoriously lacking in heat transfer coefficient correlations for non-aqueous flows (7). Unfortunately, the Groeneveld correlation was developed for steam-water flows and applying it to nitrous oxide would be completely inaccurate. The Varone and Rohsenow analytical model discussed in chapter II is ideal for this application but due to its complexity, implementing it is outside of the scope of this thesis. Instead, the Groeneveld correlation is presented here as an example of how heat transfer coefficients have been modeled in other systems. When more data becomes available for the system of interest, a similar correlation can be formulated.

The primary advantage of Groeneveld's correlation is its ease of use with the homogeneous flow model. Simply put, it is a version of the Dittus-Boelter Nusselt number correlation that is modified to include boiling effects. In this case, the correlation is given by:

$$
N u_{v}=\frac{h_{c o l d} D_{h}}{k_{v}}=a\left[\left(\frac{G D_{h}}{\mu_{v}}\right)\left(x+\frac{\rho_{v}}{\rho_{l}}(1-x)\right)\right]^{b} P r_{v}{ }^{c} Y^{d}
$$

where

$$
Y=1-0.1\left(\frac{\rho_{l}}{\rho_{v}}-1\right)^{0.4}(1-x)^{0.4}
$$


The constants a, b, c and d are empirically determined and equal to $0.00109,0.989,1.41$ and -1.15 respectively for tubes. Again, these constants are specific to water and are not applicable to the fluid of interest.

Note that the above correlation is completely empirically derived. Wojtan adapted this correlation to common refrigerants and the same method should be applied to nitrous oxide in this case. However, performing such a correlation would involve a significant amount of data that is not available at this time. For the time being, heat transfer coefficients are not modeled.

\section{IV.4.2 Final Two-Phase Model Results}

The final two-phase model as summarized above was run for standard operating conditions. These include a line pressure of 800 psia, inlet annulus pressure of 350 psia, a mass flow rate of $0.1 \mathrm{lbm} / \mathrm{sec}$, an $\mathrm{O} / \mathrm{F}$ of 2.0 and a chamber pressure of $200 \mathrm{psia}$. The predicted fluid temperatures are shown in Figure 96 below: 


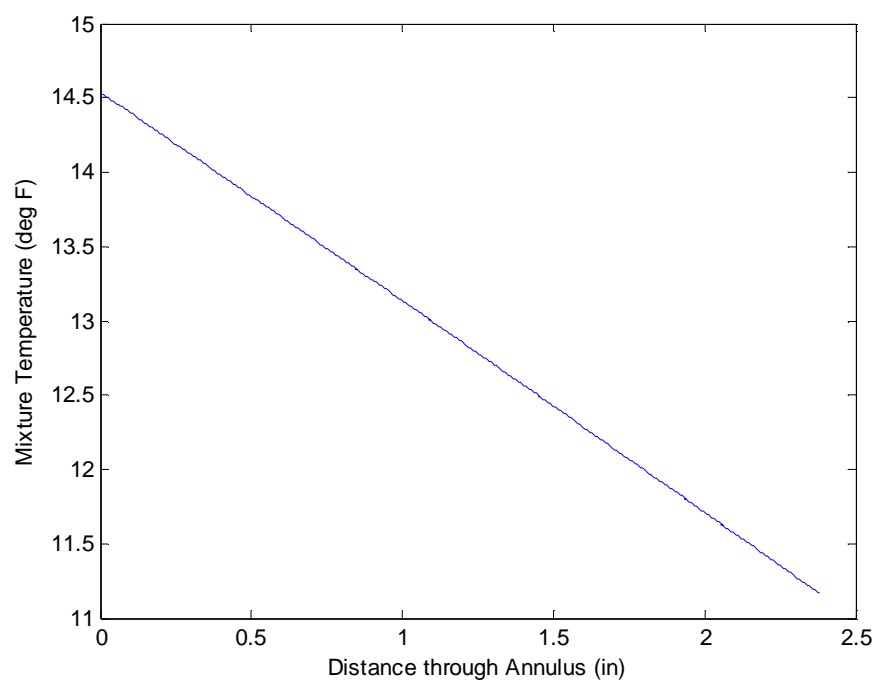

Figure 96. Mixture temperatures as predicted by the two-phase model

The predicted fluid pressures are shown in Figure 97 below:

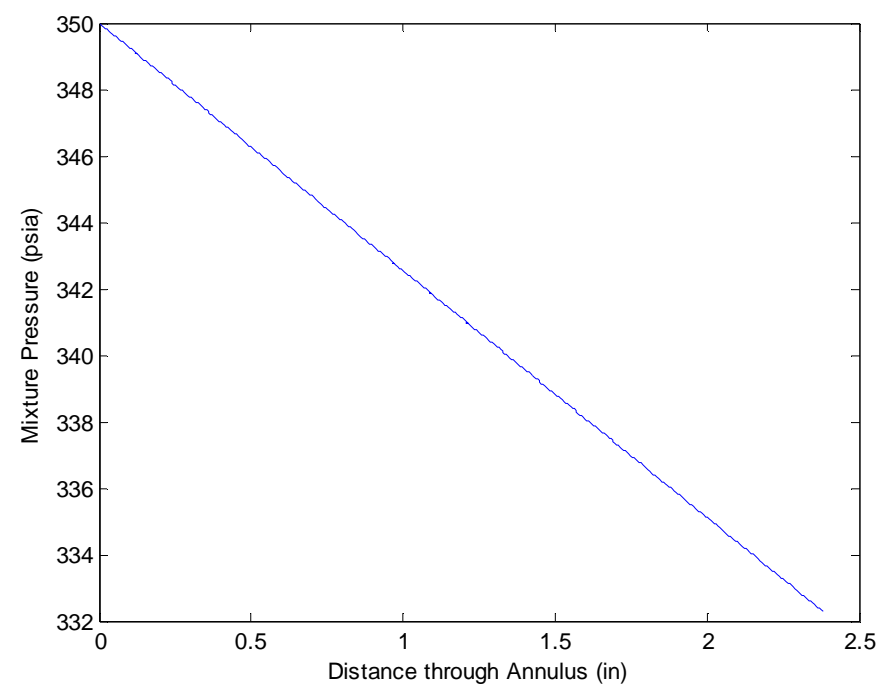

Figure 97. Mixture pressure as predicted by the two-phase model

Finally, the predicted vapor quality is shown in Figure 98 below: 


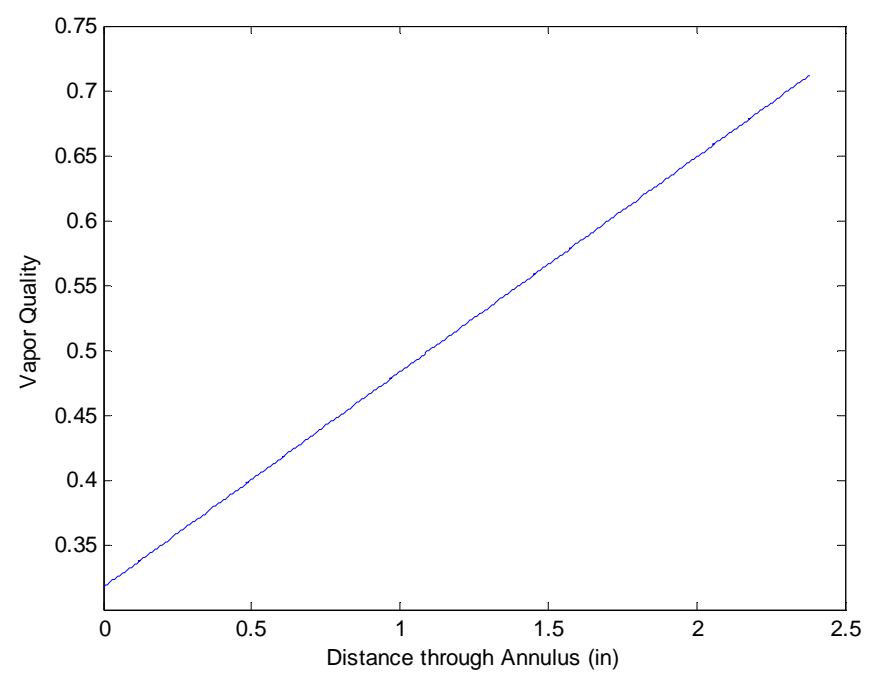

Figure 98. Quality as predicted by the two-phase model

Note that the predicted mixture temperature is significantly lower than that recorded in previous tests. This indicates that the assumption of thermal equilibrium may not be accurate.

\section{IV.5 TWO-PHASE NITROUS OXIDE EXPERIMENTS}

Two experiments were run with the Rayleigh nozzle with nitrous oxide coolant. To test repeatability of the apparatus, both tests utilized the same combustion and coolant system configurations. The combustion system included four oxidizer injectors while the coolant system used annulus inlet and outlet injectors of 0.052 " and 0.081 " respectively. The tests were done in rapid succession with only an hour of setup time in between. This was unprecedented with the Cal Poly hybrid rocket resulting in some unexpected results. First, at the end of the thirty second test, the rocket was observed to shut off unusually quickly. Upon further investigation, it was determined that a piece of fuel had separated 
from the main grain and lodged itself in the throat of the nozzle, plugging it. As this was observed to take place at the end of the experiment, it did not put into question the data collected. Secondly, the equipment was not allowed to fully cool between experiments which may have contributed to the system's ability to reach steady state. The transient data shows that the copper temperatures never fully reached steady state. In any case, the data collected in both runs is analyzed with the two-phase model presented previously in the following sections.

\section{IV.5.1 Preliminary Analysis}

The hot side conditions for both nitrous oxide cooled Rayleigh tests are shown in Table 9 below.

Table 9. Nitrous oxide Rayleigh experiment test conditions

\begin{tabular}{|c|c|c|c|c|}
\hline Run & $\begin{array}{c}\text { Chamber } \\
\text { Pressure } \\
\text { (psia) }\end{array}$ & O/F & $\begin{array}{c}\text { Combustion Gas Heat } \\
\text { Transfer Coefficient } \\
\text { (BTU/in }{ }^{2} \text { secR) }\end{array}$ & $\begin{array}{c}\text { Combustion Gas } \\
\text { Static Temperature } \\
\text { (deg F) }\end{array}$ \\
\hline 68 & 140.2 & 1.99 & $2.25 \mathrm{E}-03$ & 2962 \\
\hline 69 & 147.5 & 2.05 & $2.15 \mathrm{E}-03$ & 3062 \\
\hline
\end{tabular}

The Revellin Thome dryout model was first run with the appropriate inputs. For the first and second $\mathrm{N}_{2} \mathrm{O}$ cooled tests, dryout was predicted 9.5 and 9.7 millimeters into the annulus respectively. Because this is less than $25 \%$ into the annulus, the annular flow entrance length was neglected and mist flow was modeled throughout the annulus. Accordingly, the homogeneous flow model was utilized to model the fluid pressure and temperature. 


\section{IV.5.2 First Nitrous Oxide Cooled Experiment Results}

A total of 131 seconds of data was collected in Run 68. The time period between 50 and 65 seconds was judged to be steady state based on the relative invariability of the copper temperatures in this region. The transient plots of copper and fluid temperatures as well as fluid pressure and mass flow rate in the steady state region are given below:

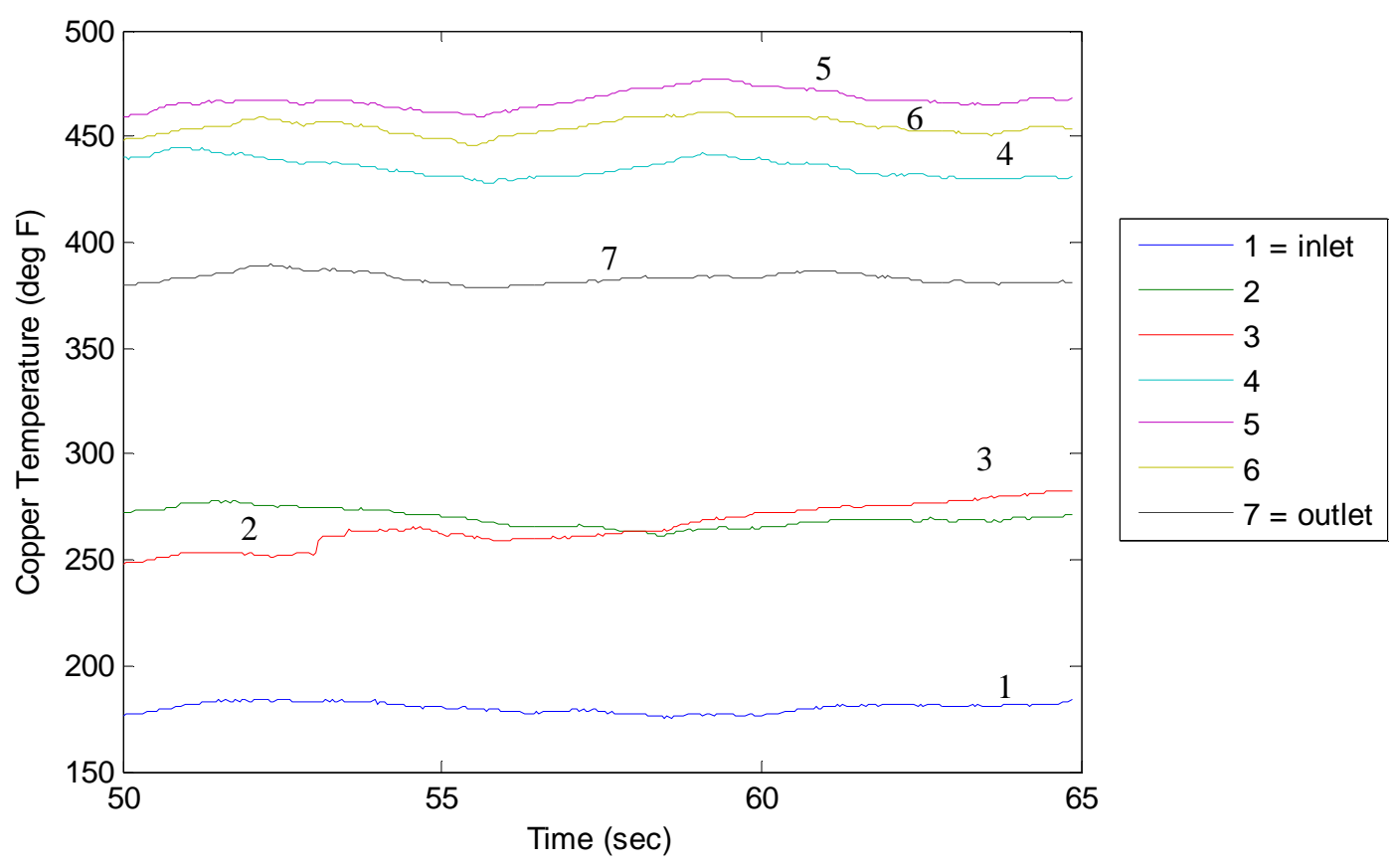

Figure 99. Nitrous oxide test 1: transient copper temperatures (Run 68) 


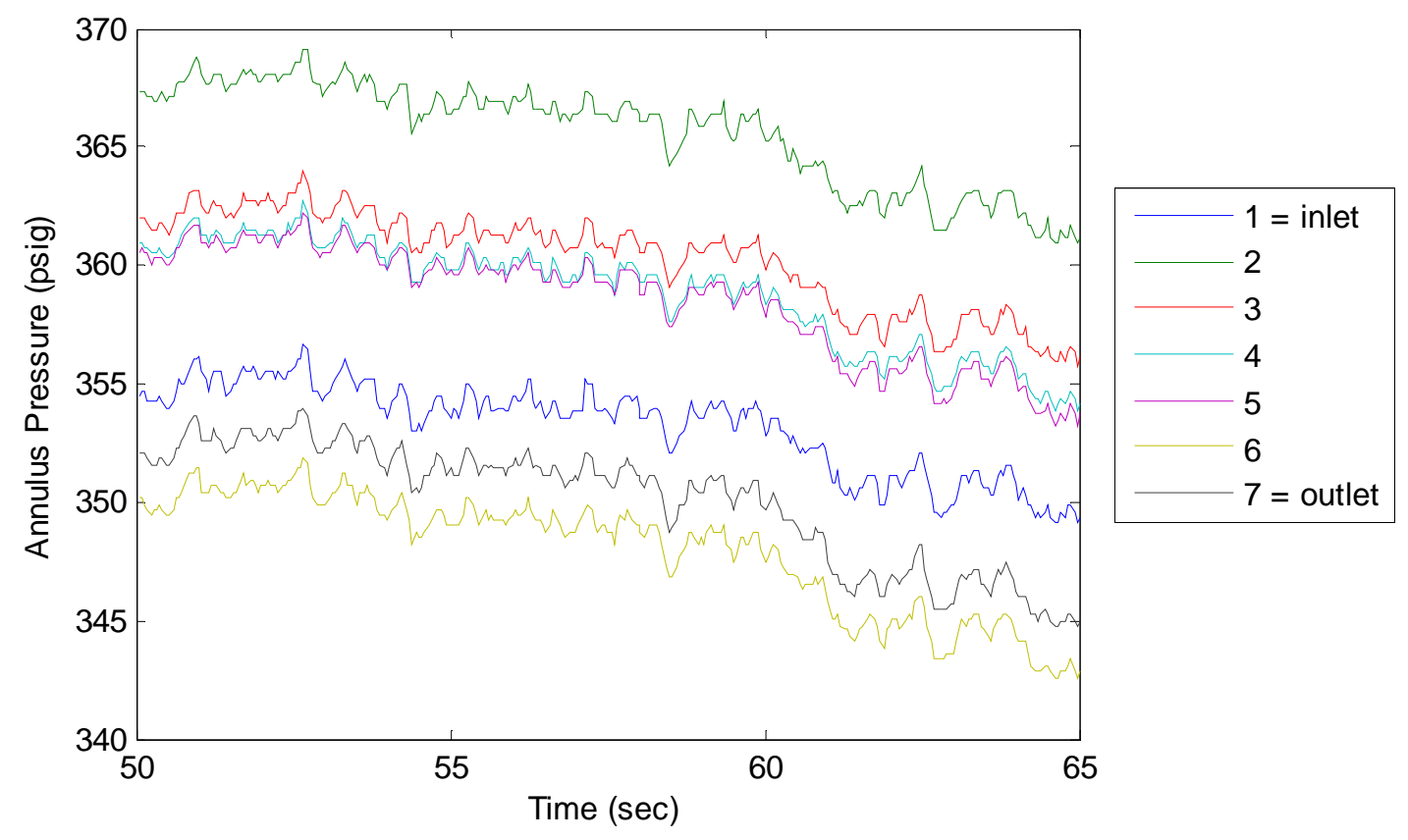

Figure 100. Nitrous oxide test 1: transient annulus pressures (Run 68)

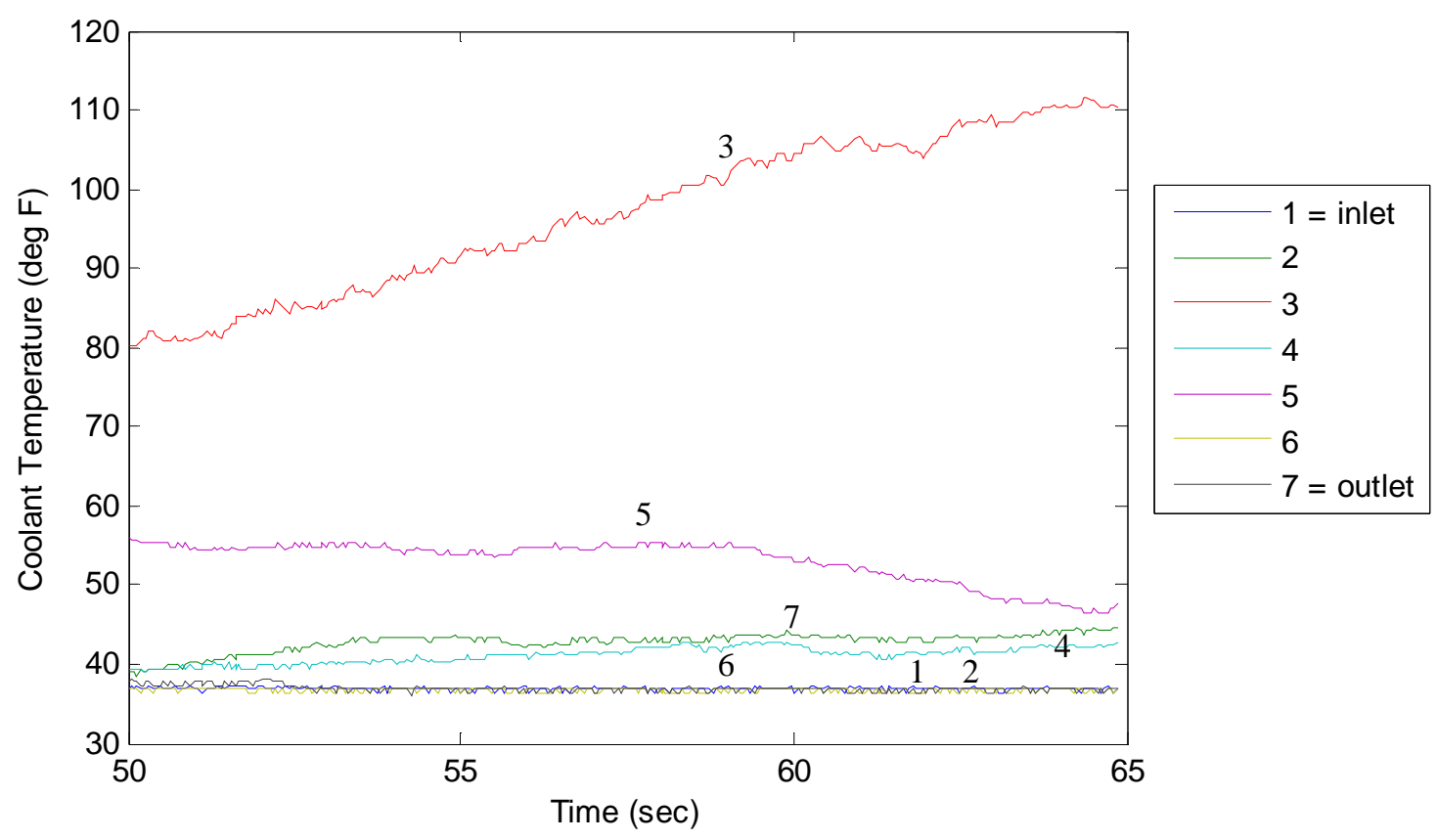

Figure 101. Nitrous oxide test 1: transient fluid temperatures (Run 68) 


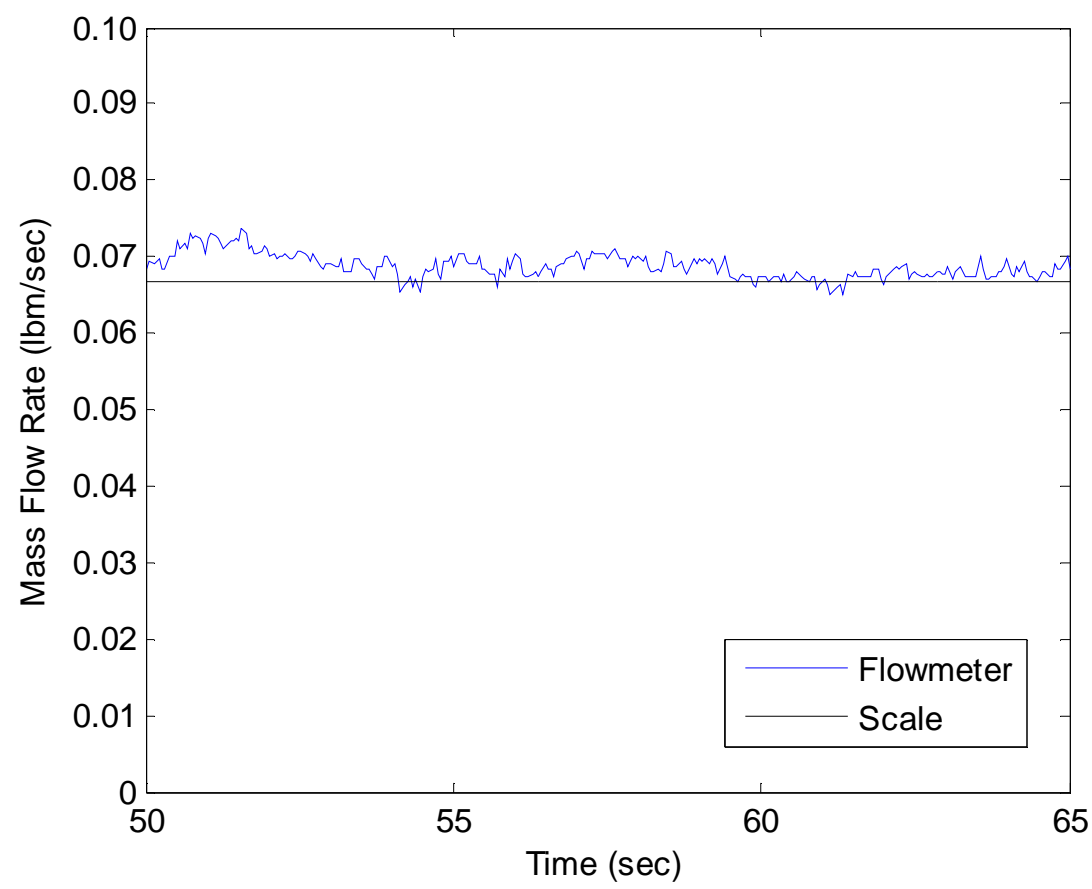

Figure 102. Nitrous oxide test 1: transient scale and flow meter mass flow rates (Run 68)

Several comments should be made about the measured fluid temperatures. First, unexpectedly high temperatures were measured by the third thermocouple from the inlet to the annulus. Prior to running the test, the coolant system was tested without the presence of a heat load. In this test, no inconsistencies were noticed in the thermocouple readings. These tests gave no indication of the unusually large temperature sensed by the third thermocouple. This coolant system test was repeated after the nitrous oxide cooled experiment. Again, this test did not show any abnormal behavior of thermocouple three. This is shown in Figure 103 below: 


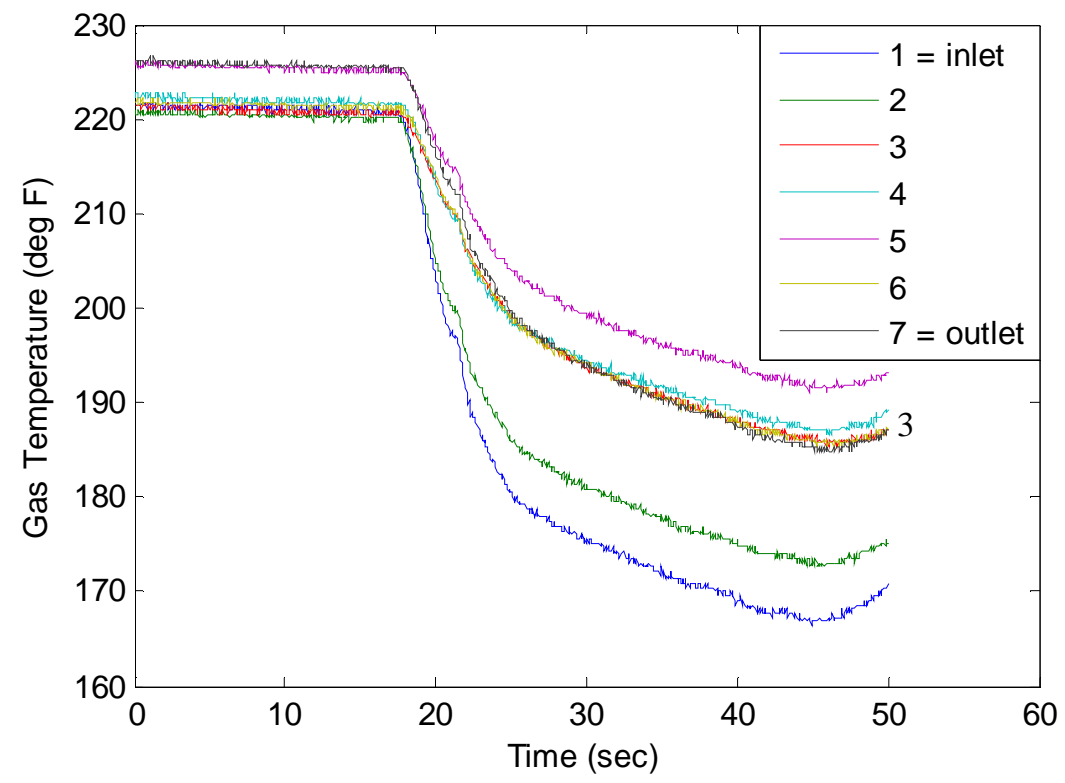

Figure 103. Thermocouple 3 behaves as expected without a heat load

Based on these results, it is presumed that the third thermocouple has some contact with the copper during the hot fire and therefore is not indicative of the coolant temperature alone. This thermocouple reading will be considered an outlier in subsequent tests. Also, notice that the fifth and seventh thermocouples are offset by approximately five degrees. This suggests that in the previous and subsequent tests, these two temperatures will read five degrees higher than the physical temperature. These issues will add error to the experimental results.

Referring again to Figure 101, notice that many of the temperatures seem to have "flatlined" at approximately $36^{\circ} \mathrm{F}$. After additional scrutiny, it was noted that the minimum temperature reading possible from the coolant thermocouples was $36^{\circ} \mathrm{F}$. For the readings in which this value is displayed, it is almost certain that the fluid temperature was below 
$36^{\circ} \mathrm{F}$ and the equipment was unable to monitor its temperature. This too will be taken into consideration when comparing the model to the experimental data.

The time averaged experimental properties through the annulus along with the homogeneous flow model predictions are shown in Figure 104 below. Note that the error bars presented on experimental data represents the resolution of the equipment used to measure the temperatures and pressures.

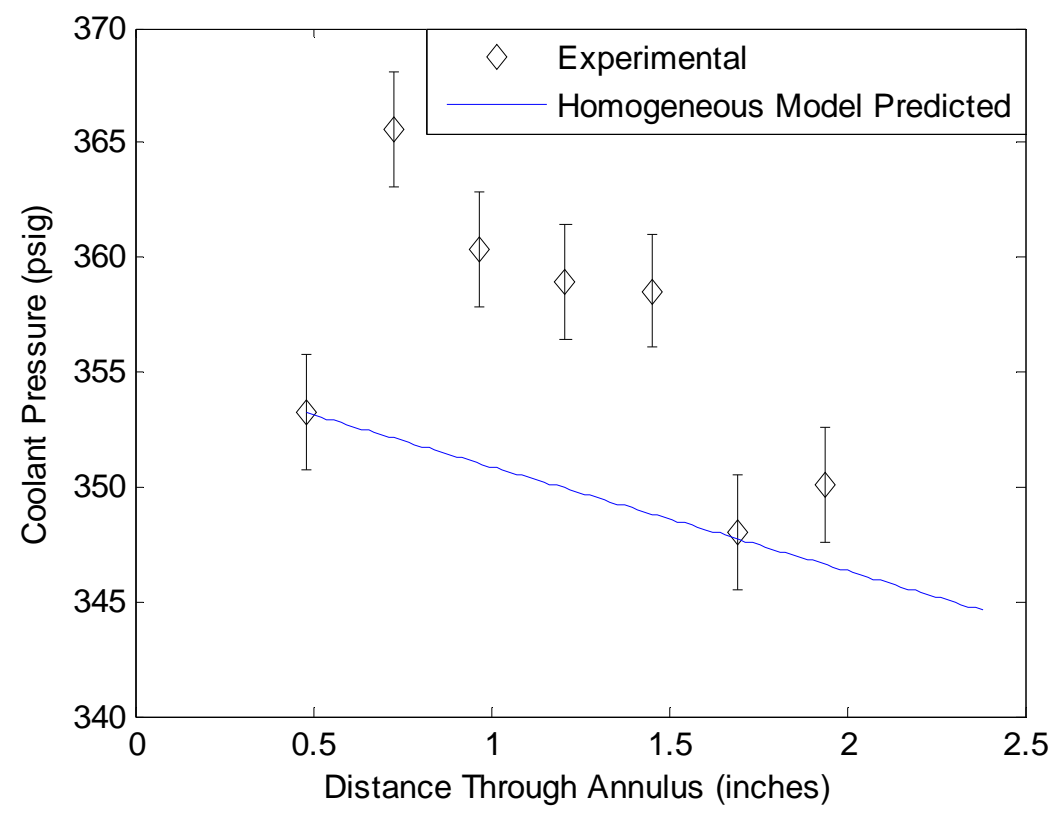

Figure 104. $\mathrm{N}_{2} \mathrm{O}$ test 1: steady state fluid pressures 


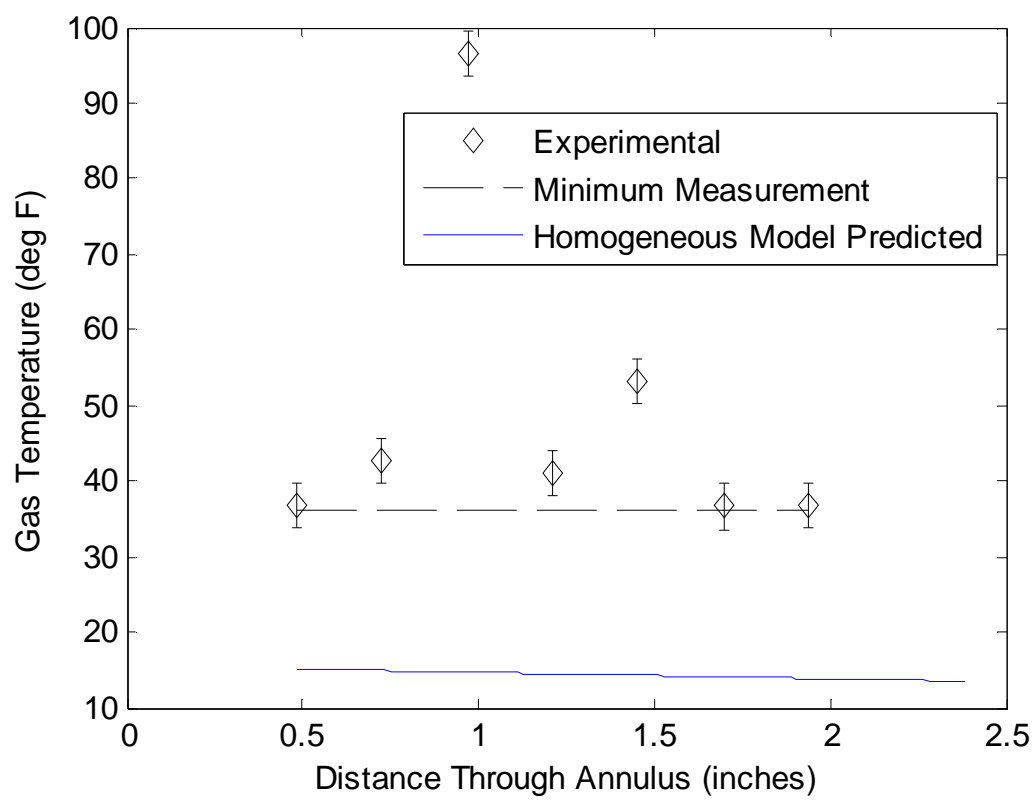

Figure $105 . \mathrm{N}_{2} \mathrm{O}$ test 1: steady state fluid temperature

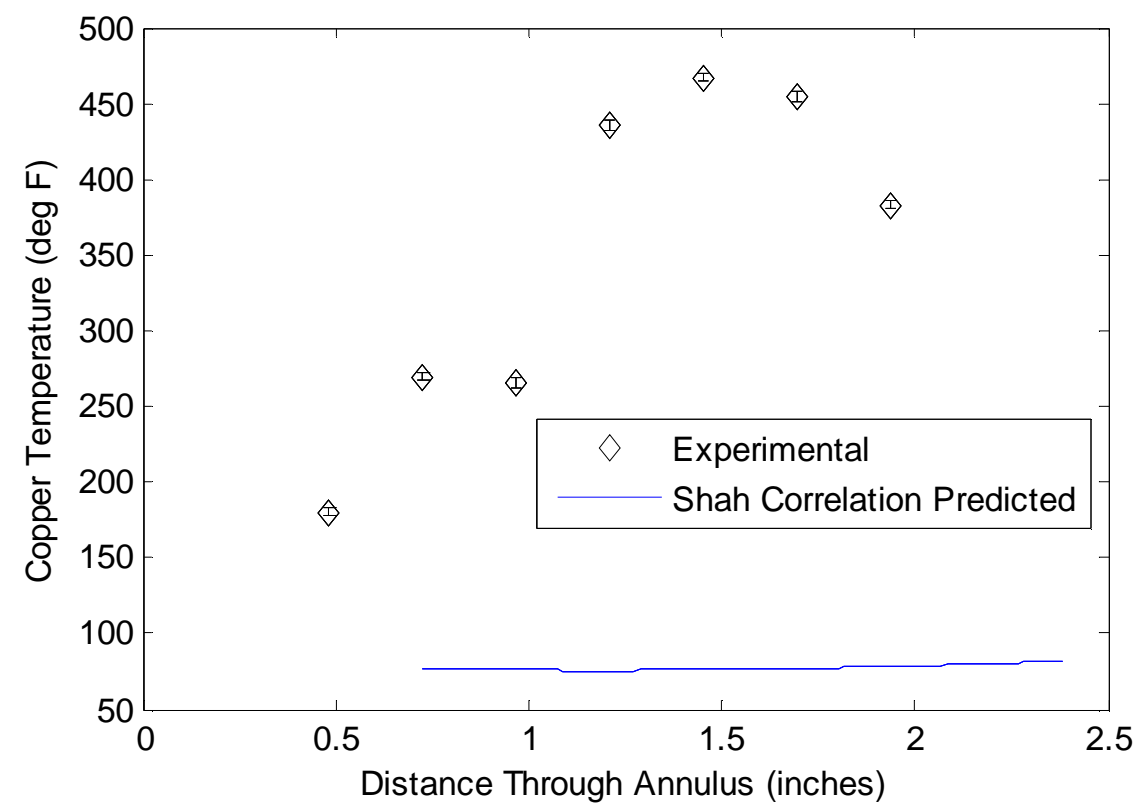

Figure $106 . \mathrm{N}_{2} \mathrm{O}$ test 1 : steady state copper temperatures 


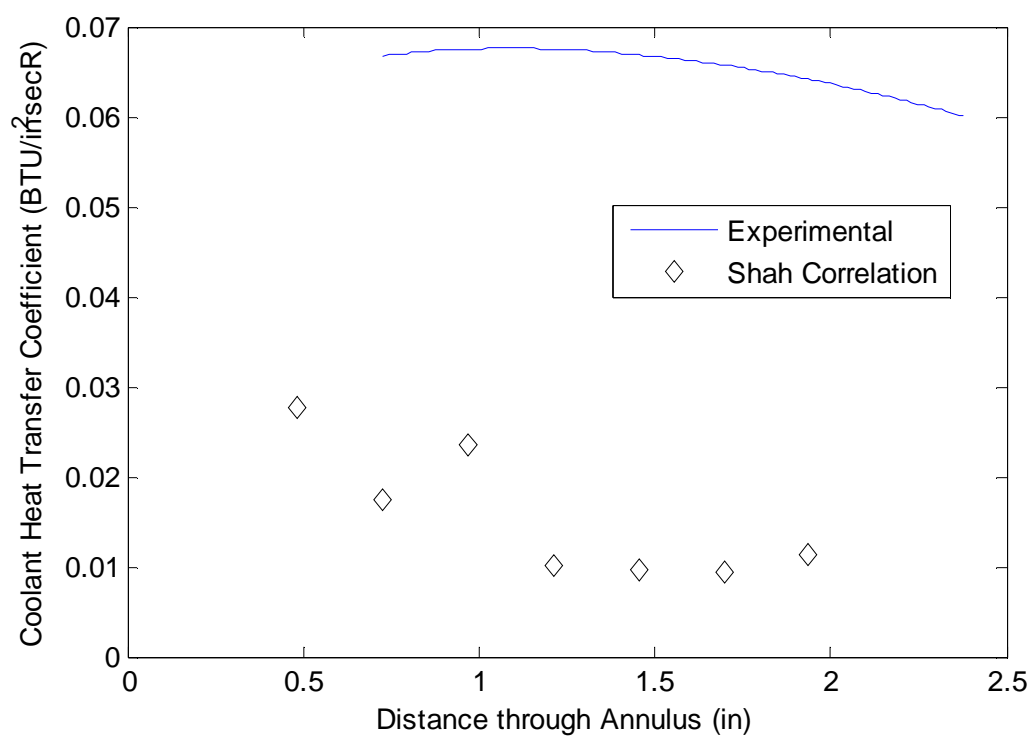

Figure 107. $\mathrm{N}_{2} \mathrm{O}$ test 1: measured and predicted heat transfer coefficients

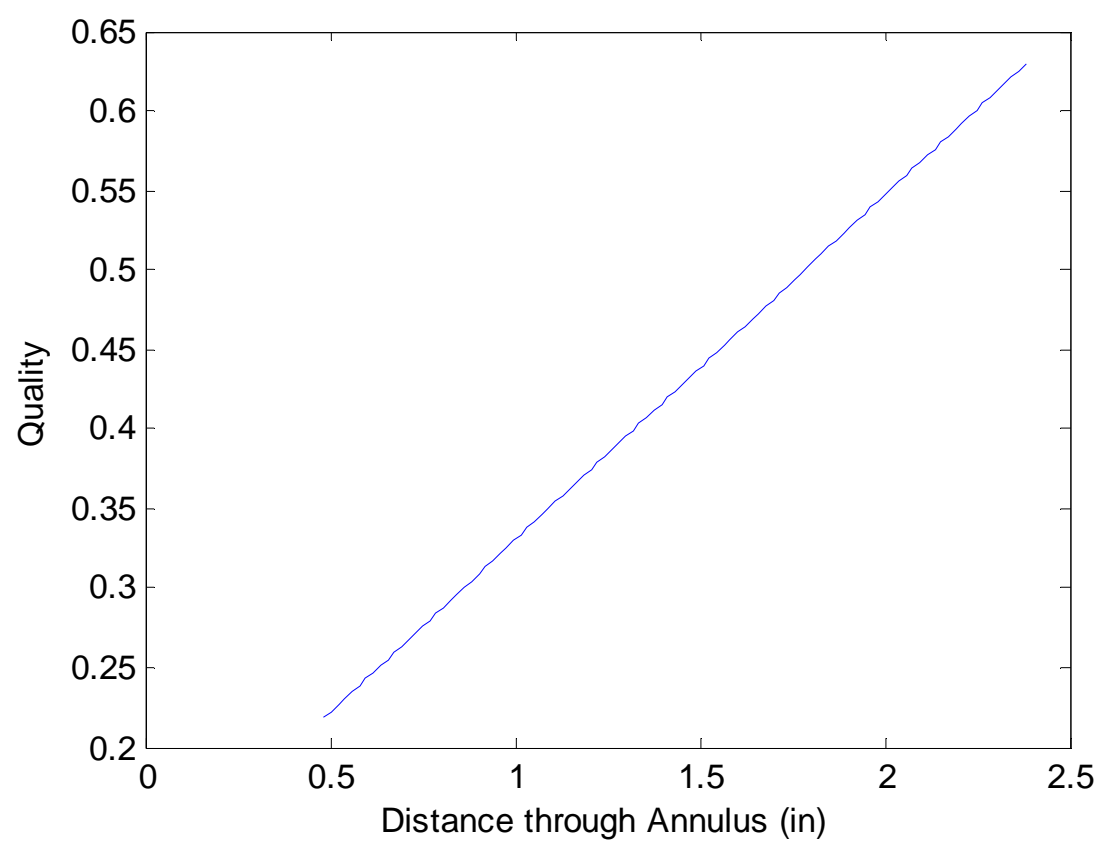

Figure $108 . \mathrm{N}_{2} \mathrm{O}$ test 1 : thermodynamic quality through the annulus

The results presented above will be discussed in subsequent sections. 


\section{IV.5.3 Second Nitrous Oxide Cooled Experiment Results}

As was mentioned previously, the second nitrous oxide cooled test utilized identical combustion and coolant system setups to the first. The test was run for 131 seconds. The time between 63 and 72 seconds was judged as steady state from the copper temperature profiles. The transient plots of pressure, temperature and mass flow rate appear below:

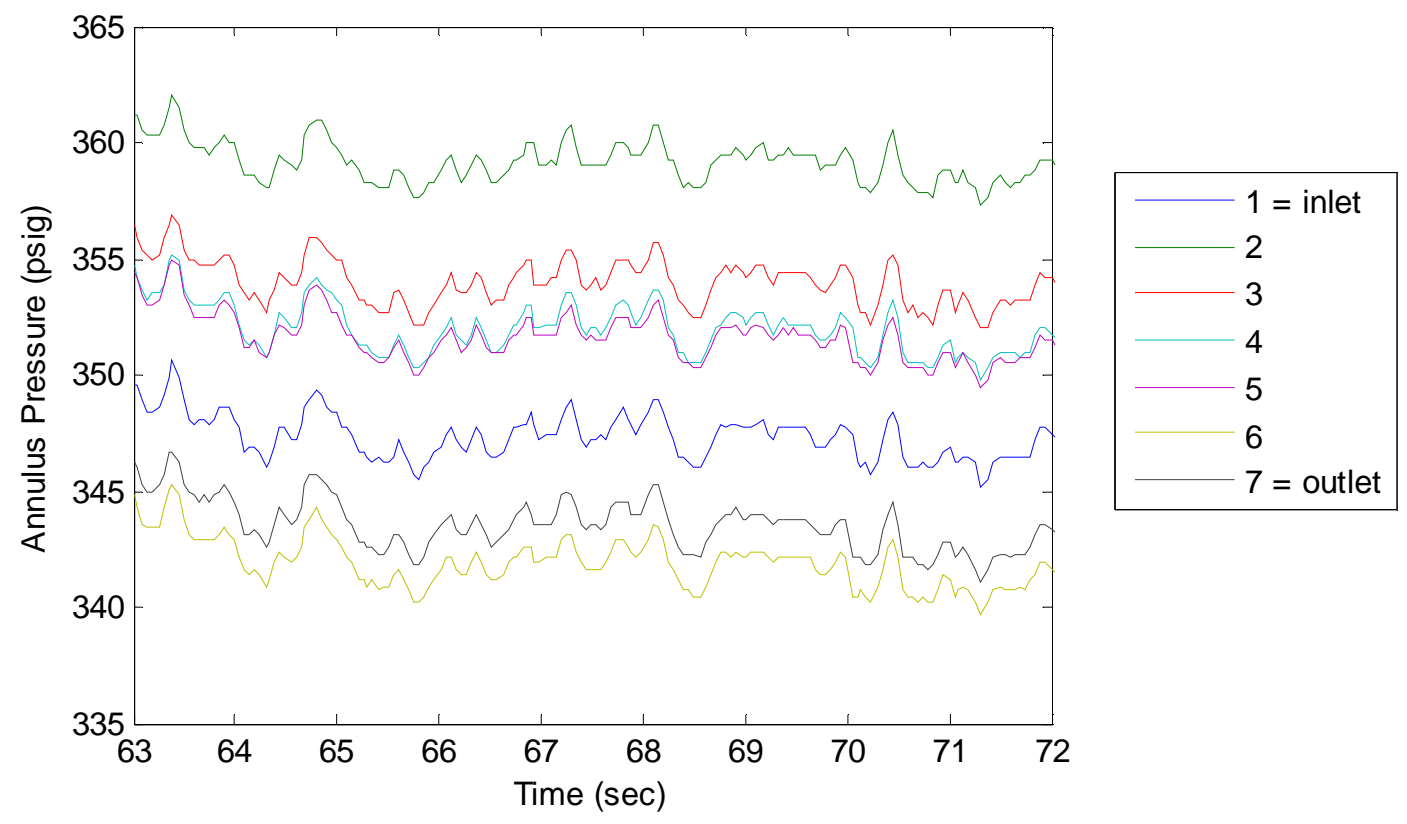

Figure 109. $\mathrm{N}_{2} \mathrm{O}$ test 2: transient coolant pressures in steady state region (Run 69) 


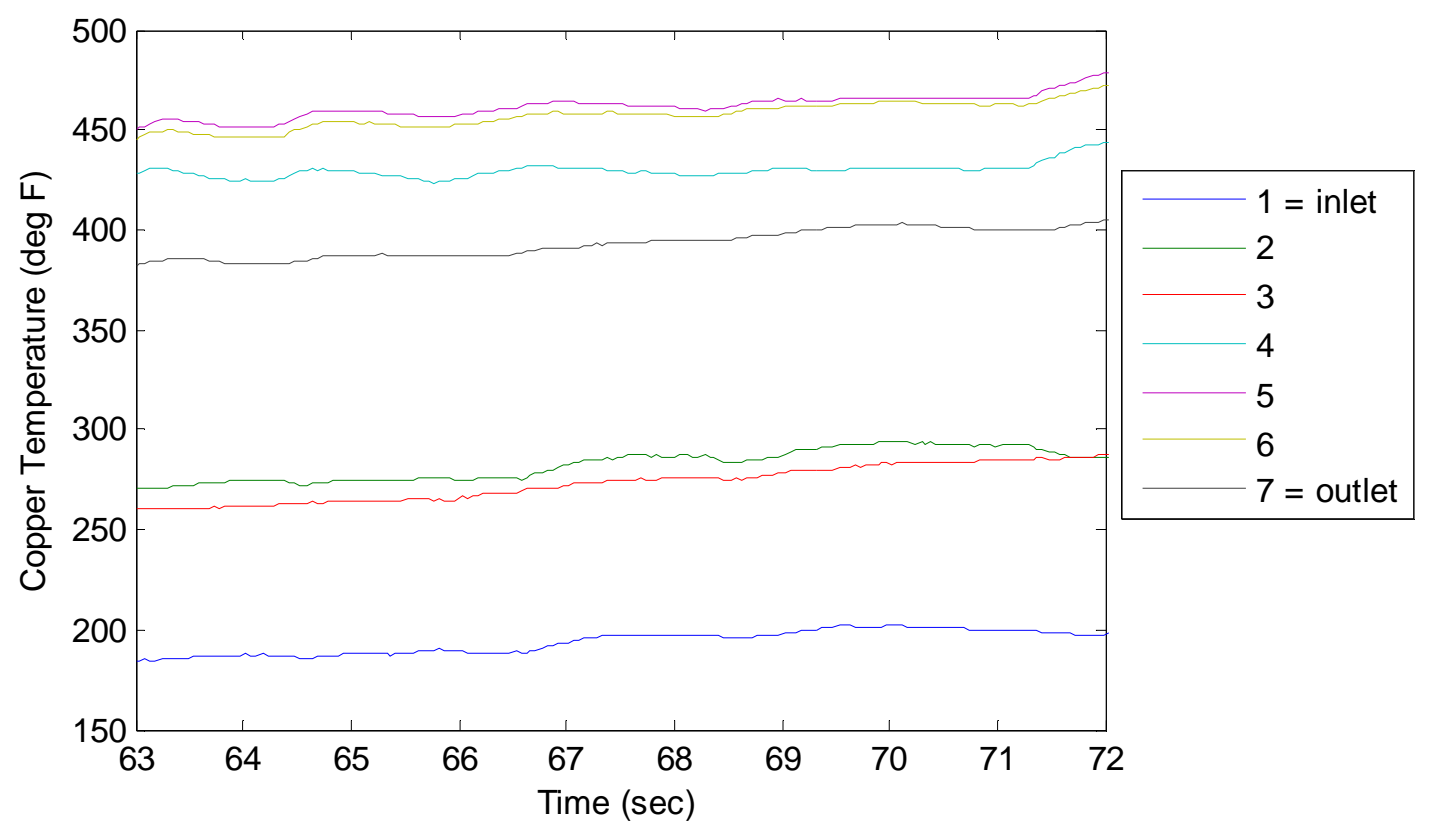

Figure 110. $\mathrm{N}_{2} \mathrm{O}$ test 2: transient copper temperatures in steady state region (Run 69)

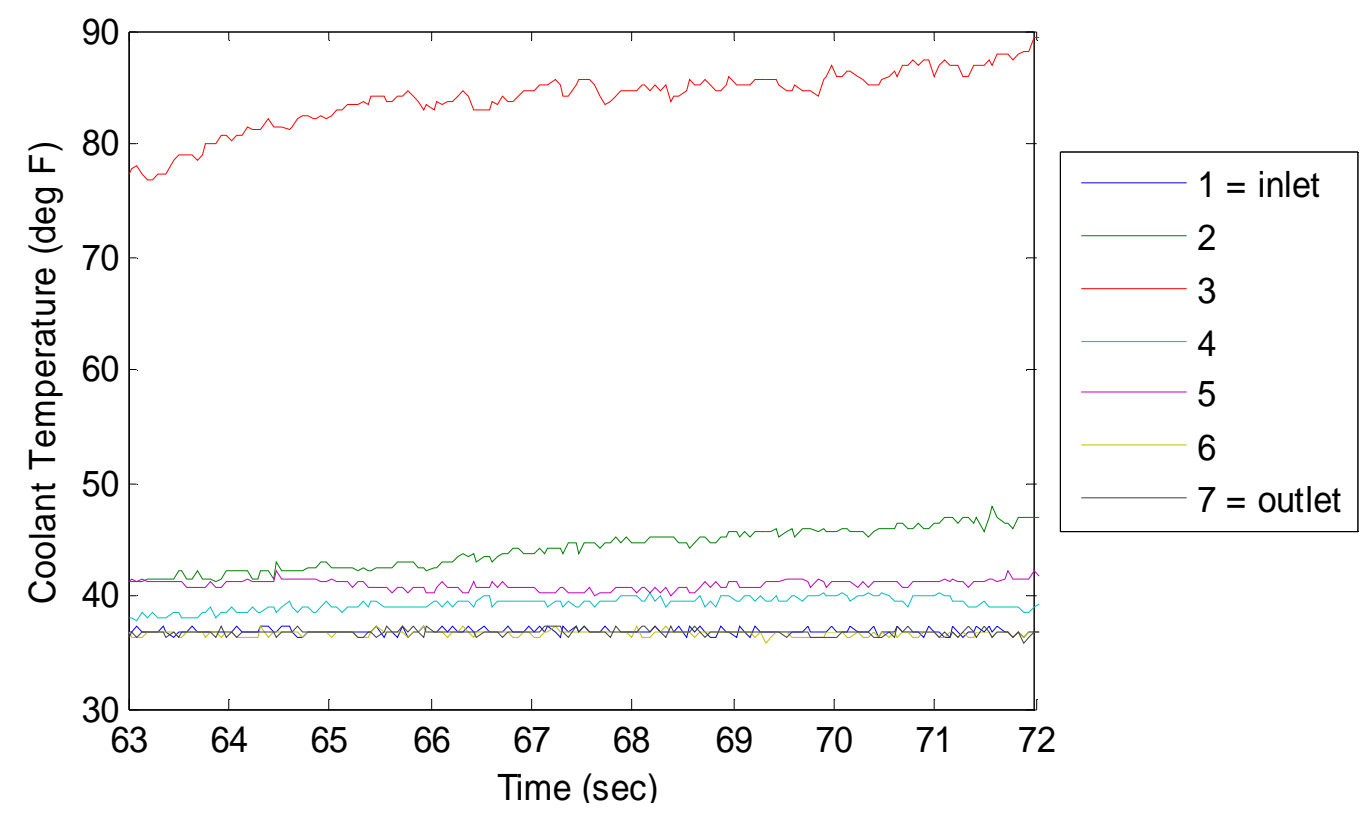

Figure $111 . \mathrm{N}_{2} \mathrm{O}$ test 2: transient coolant temperatures in steady state region (Run 69) 


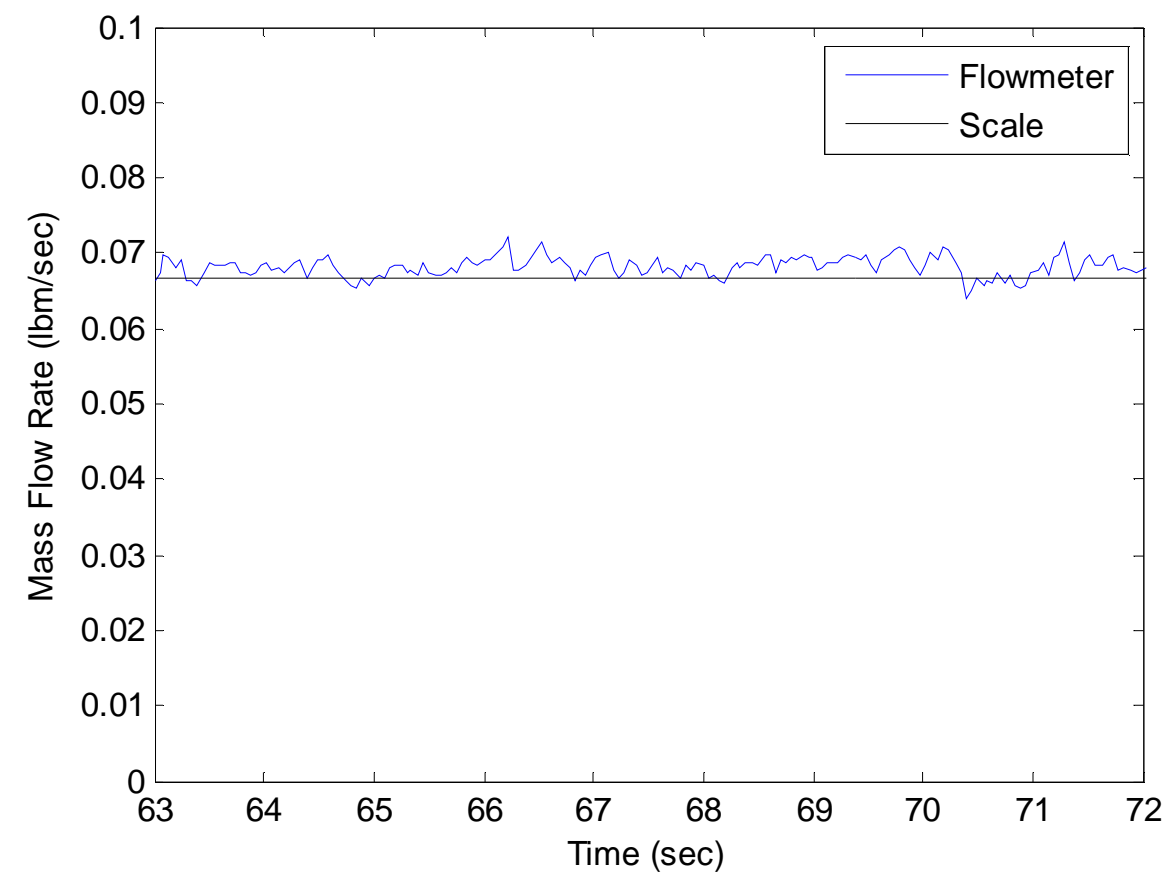

Figure 112. $\mathrm{N}_{2} \mathrm{O}$ test 2: transient mass flow rate in steady state region (Run 69)

Note that the third coolant thermocouple is again reading significantly higher than expected as was the case in the previous test. Based on the tests run after the first nitrous oxide cooled run, this inconsistency is assumed to be caused by the equipment. The transient plots shown above are averaged between 63 and 72 seconds to give steady state values. These values are plotted through the annulus in the following figures. Note that the error bars in the figures below represent the approximate accuracy of the measurement device (i.e., pressure transducer or thermocouple). 


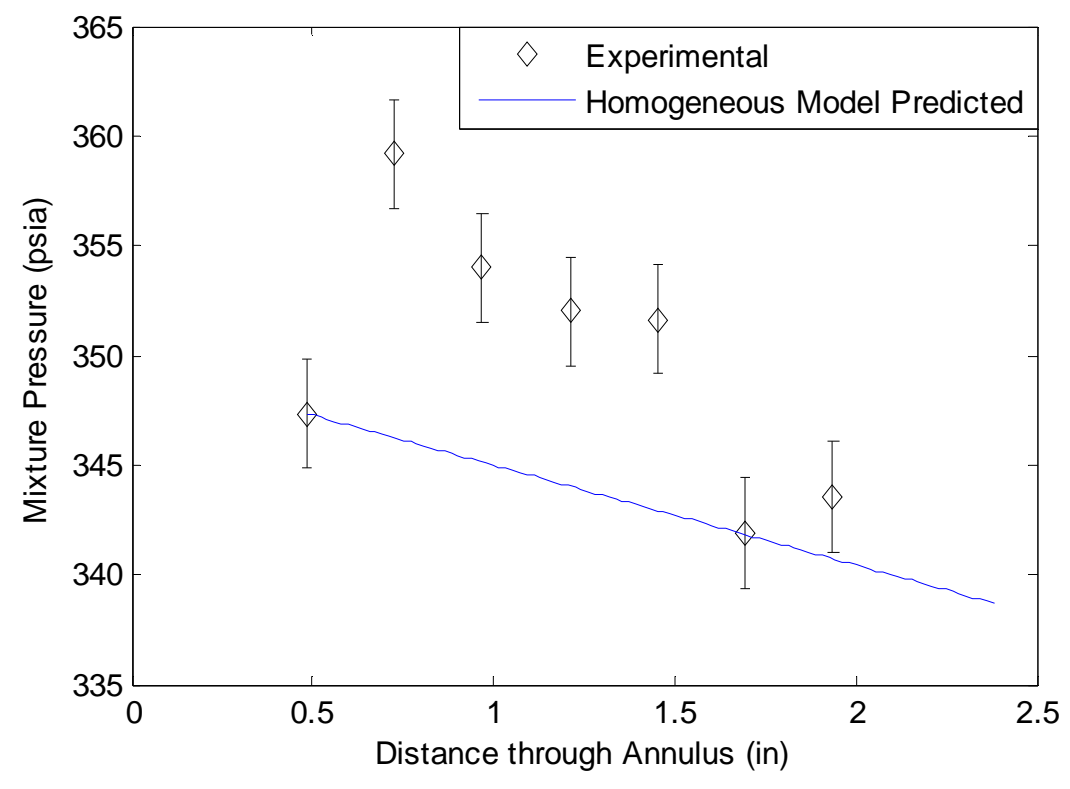

Figure $113 . \mathrm{N}_{2} \mathrm{O}$ test 2 : steady state coolant pressures

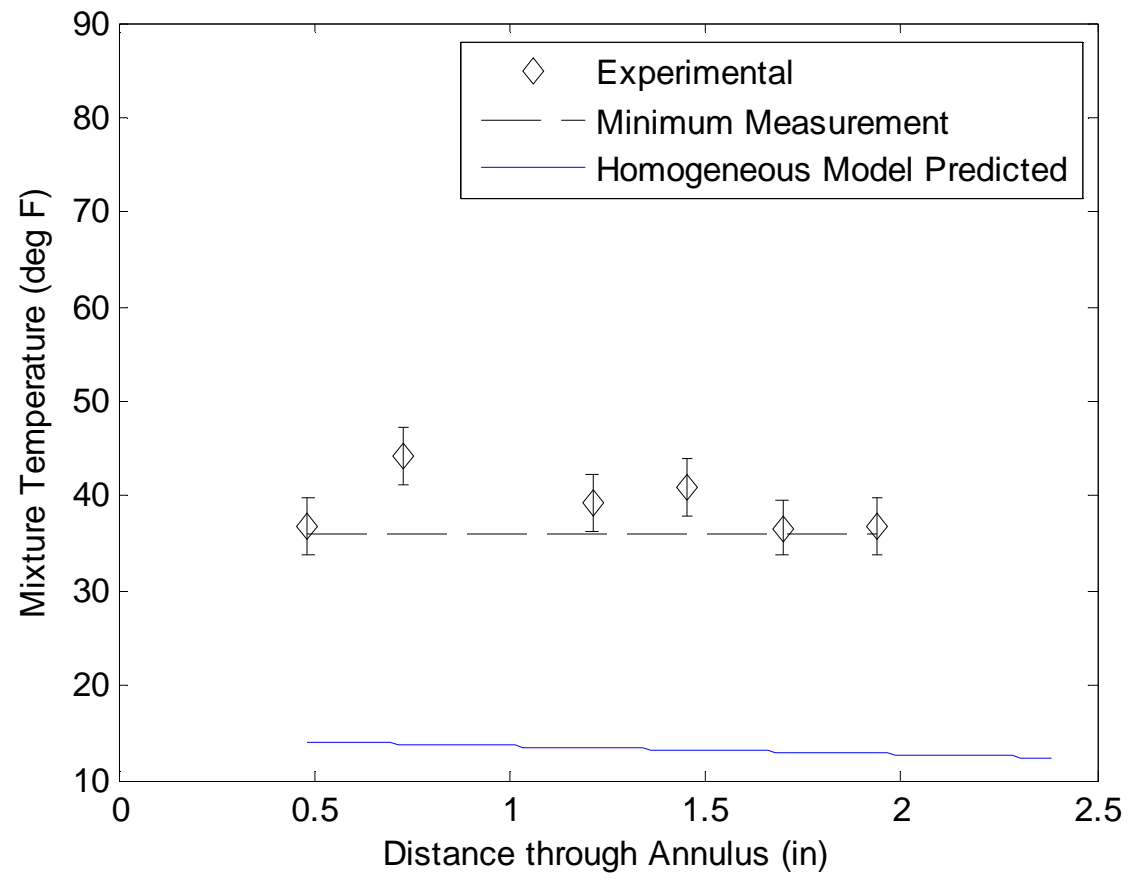

Figure $114 . \mathrm{N}_{2} \mathrm{O}$ test 2 : steady state coolant temperatures 


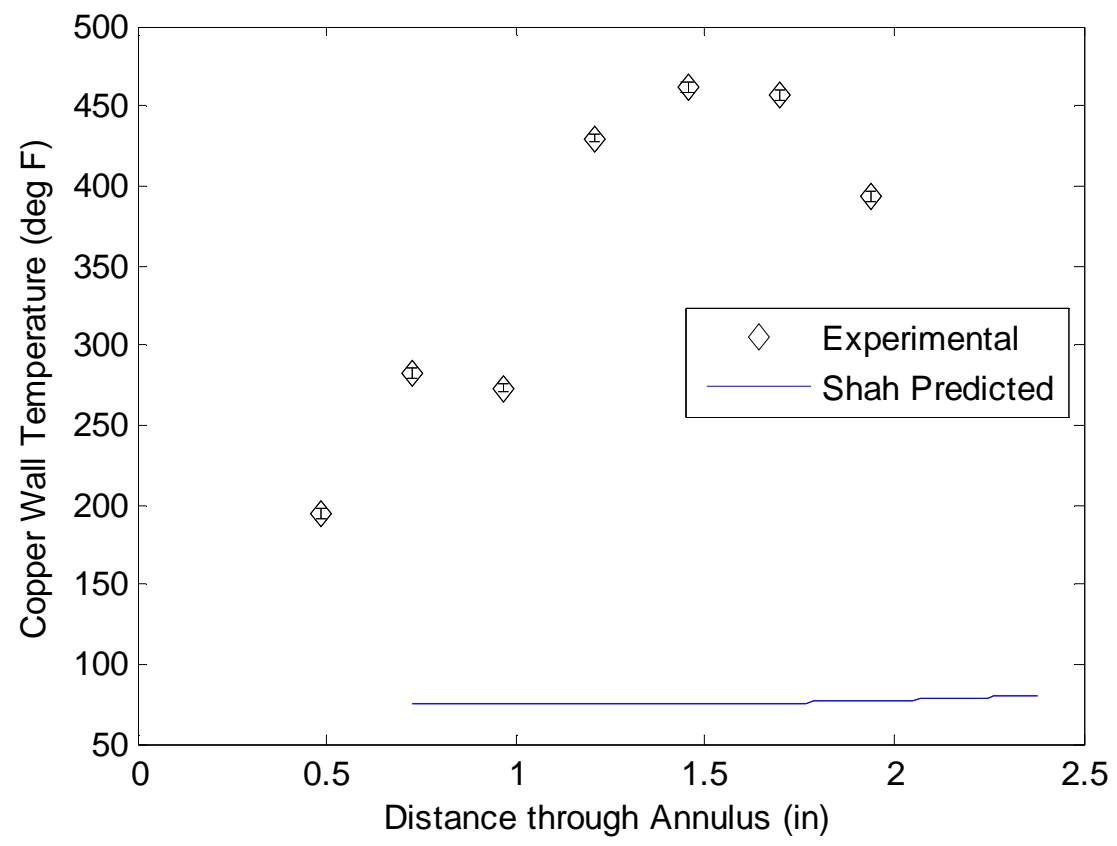

Figure $115 . \mathrm{N}_{2} \mathrm{O}$ test 2 : steady state copper temperatures

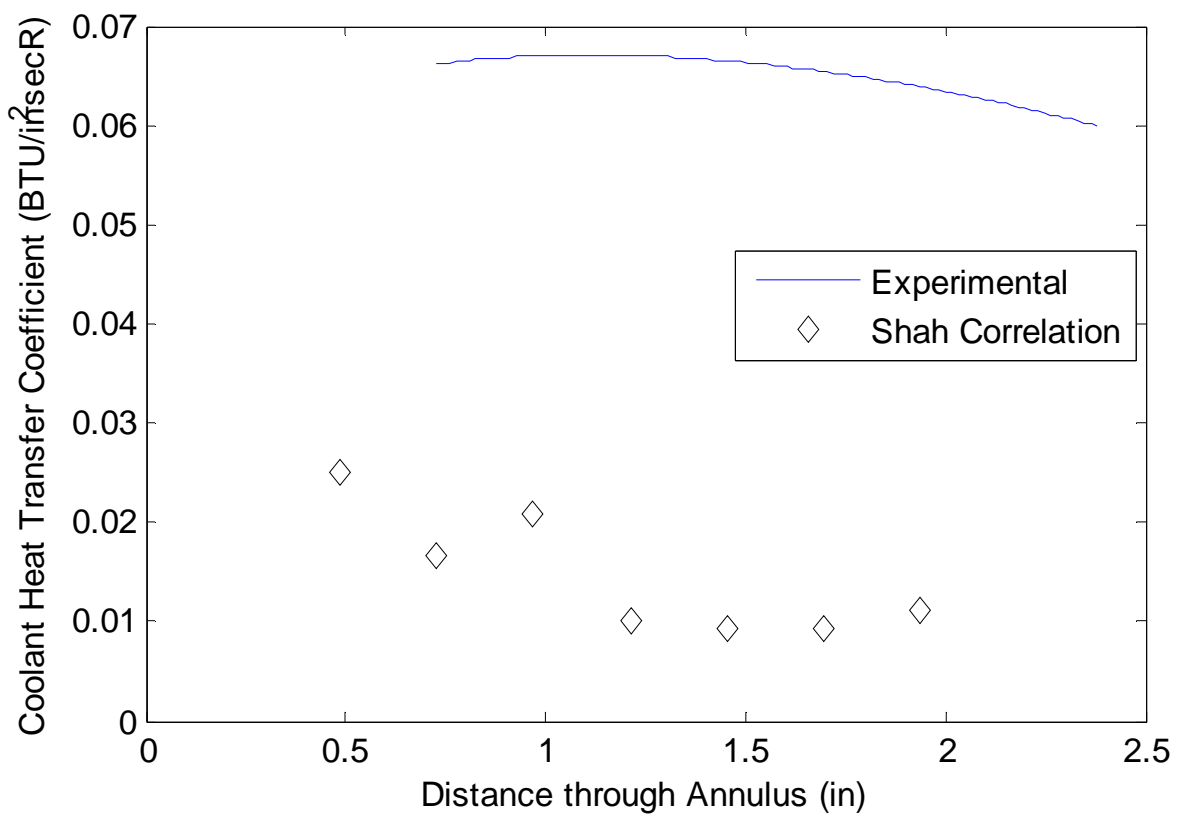

Figure 116. $\mathrm{N}_{2} \mathrm{O}$ test 2: measured and predicted coolant heat transfer coefficients 


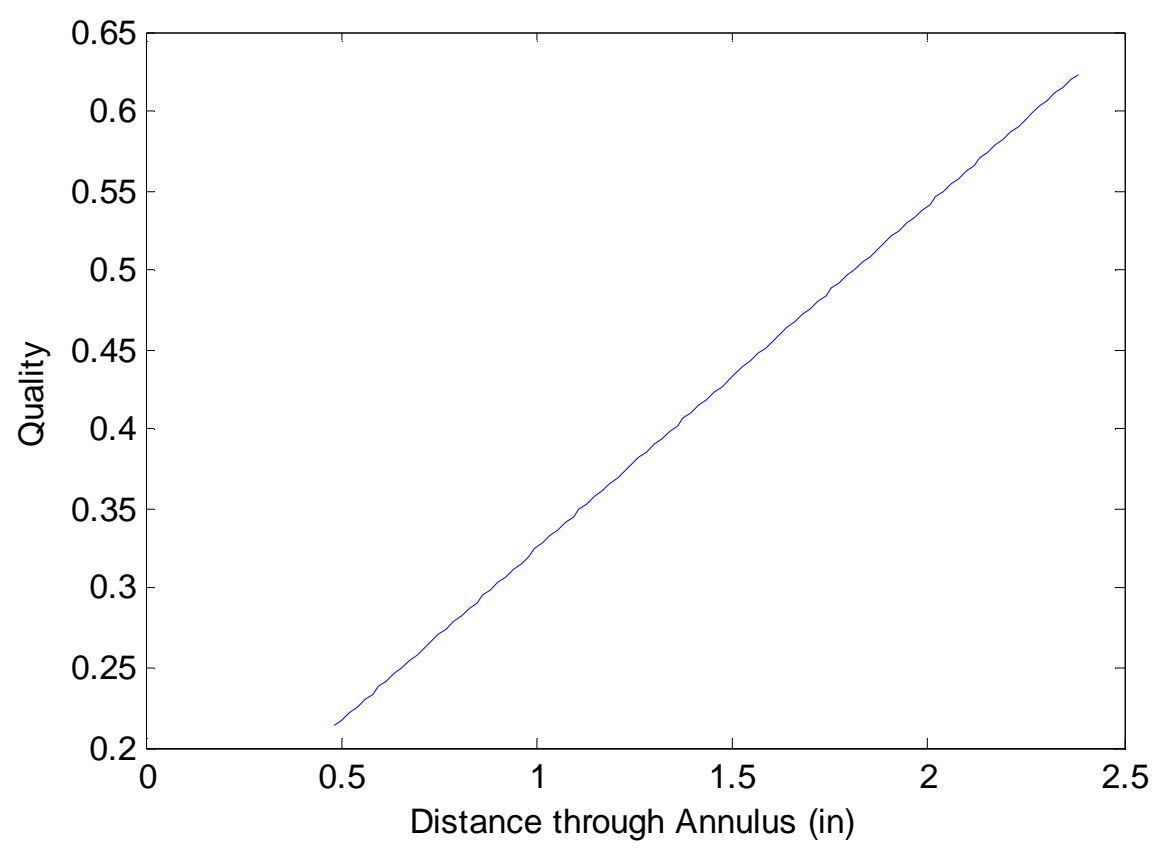

Figure 117. $\mathrm{N}_{2} \mathrm{O}$ test 2 : thermodynamic quality

\section{IV.6 TWO-PHASE RESULTS DISCUSSION}

First, note the striking similarities between the two nitrous oxide cooled experiments. This is advantageous as it suggests that none of the data collected represents some sort of anomaly. However, note that any systematic equipment malfunction or inaccuracy would affect both test results and it is therefore still possible that these may exist.

It should also be noted that the heat transfer coefficients and wall temperatures predicted by the Shah correlation are plotted with the measured experimental data. Notice that the Shah correlation significantly overpredicts the heat transfer coefficients and 
underpredicts the wall temperatures. This suggests that the coolant does not flow in the nucleate boiling regime, supporting the findings of the dryout model.

Several other interesting occurrences should be discussed. These include: (1) input parameter selection, and (2) coolant temperature discrepancies. Recall that the first listed item was also discussed in the helium results section.

\section{IV.6.1 Input Parameter Selection}

Recall that the two-phase Rayleigh model requires the temperature and pressure at the inlet of the annulus as inputs. The ideal gas experiments showed the possible recirculation and other entrance effects affected the characteristics of the flow at the annulus inlet. The two-phase model was rerun for both nitrous oxide cooled tests with the properties measured at the second port as inputs. The measured values at the second port were used as inputs in this case because the ideal gas experiments showed that inlet measurement port is likely located in an entrance region. Because the two-phase models assume fully developed flow and do not account for entrance effects, the second measurement port is a more appropriate initial condition. The results are shown in Figure 118 and Figure 119 below. As was the case previously, the error bars represent the approximate resolution of the measurement device (i.e., pressure transducer or thermocouple). 


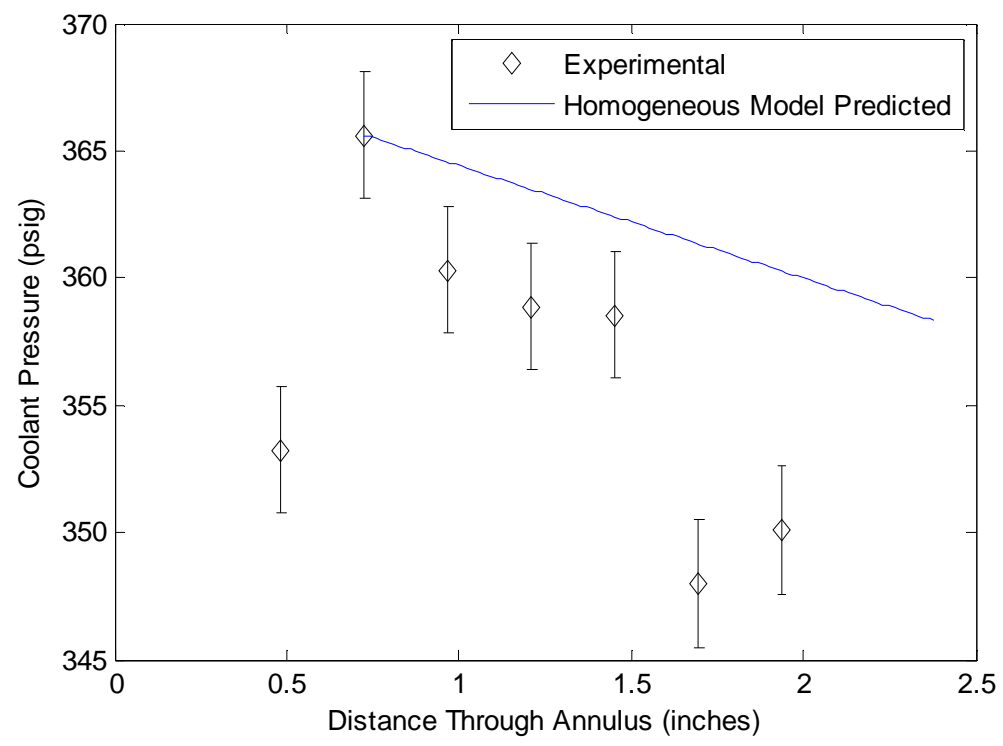

Figure 118. $\mathrm{N}_{2} \mathrm{O}$ test 1: coolant pressures with second measurement as input

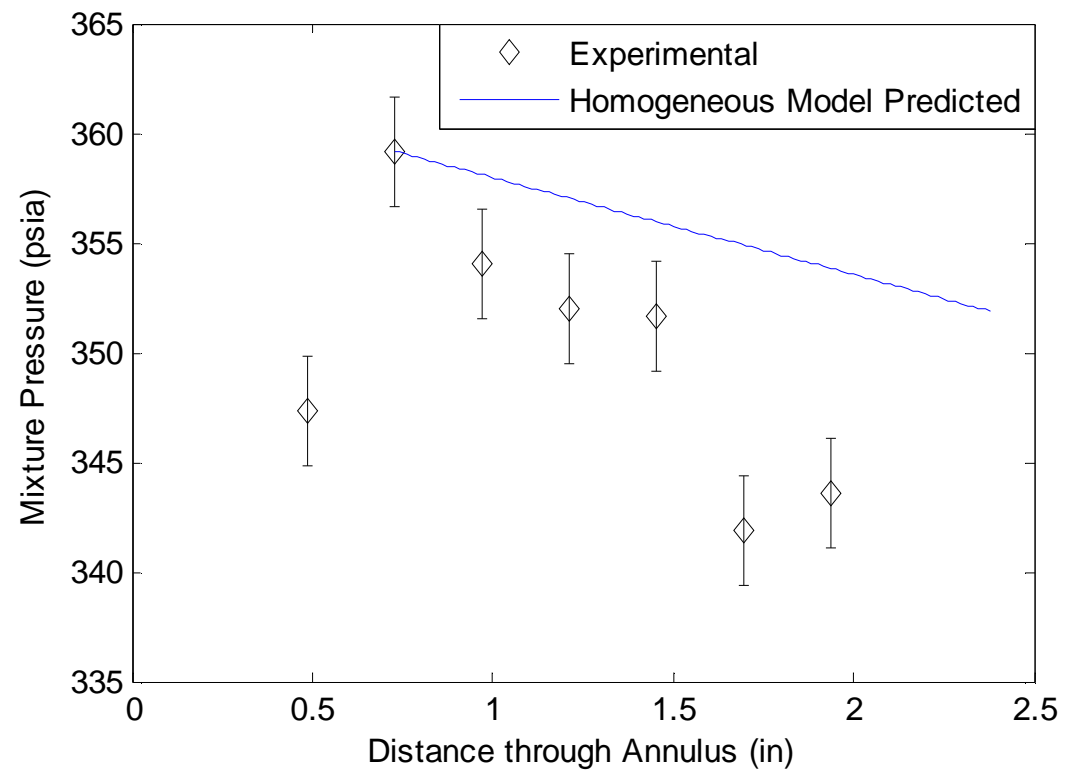

Figure 119. $\mathrm{N}_{2} \mathrm{O}$ test 2: coolant pressures with second measurement as input

Note that the two-phase Rayleigh model does not account for frictional losses. This is likely the cause of the difference between the experimental values and model prediction. Similar to the ideal gas case, a Fanno model may be useful in better predicting the 
pressure drops. In any case, the similarities between the ideal gas results and those shown above are reassuring. It suggests that the unusually low pressures at the inlet and outlet of the annulus are not related to the two-phase nature of nitrous oxide but rather the complicated geometry of the cooling annulus.

\section{IV.6.2 Coolant Temperature Discrepancies}

Several problems were encountered with the measured coolant temperatures. As was discussed earlier, several of the coolant thermocouples read the minimum temperature, indicating that the physical fluid temperature was below $36^{\circ} \mathrm{F}$. However, some of them did read above this value which suggests that the coolant temperatures existed somewhere around the equipment cutoff. Despite this equipment malfunction, it is likely that the thermal equilibrium model grossly underpredicts the coolant temperatures. In order to correct for this, some method of accounting for thermal non-equilibrium is necessary. The Varone and Rohsenow model is ideal for this scenario but because it is so complex to implement, it is outside of the scope of this thesis.

\section{IV.7 TWO-PHASE MODELING CONCLUSIONS}

Overall, the homogeneous flow model does a satisfactory job at describing the behavior of $\mathrm{N}_{2} \mathrm{O}$ in the hybrid rocket coolant system. The similarities between the results of the ideal gas and two-phase experiments are reassuring as it indicates that very few to no abnormalities are being introduced by the two-phase coolant alone. For the full predictive model to come to fruition, additional effort is necessary. First, the two-phase homogeneous Rayleigh model alone does not account for the full pressure drop seen 
through the annulus. As was done for the ideal gas, a complimentary Fanno model should be developed to account for two-phase frictional losses. Second, the assumption of thermal equilibrium results in underprediction of coolant temperatures. A method such as the Varone and Rohsenow model would be beneficial and provide additional accuracy. It may also be beneficial to develop methods of predicting entrance and exit characteristics. 


\section{CONCLUSIONS AND RECOMMENDATIONS}

Varone showed that analytically modeling two-phase mist flow in a straight vertical tube is by no means trivial (6). Novak showed that even the slightest change in the experimental parameters of two-phase flow in a vertical round tube can significantly affect the heat transfer characteristics (23). In this case, the system of interest is extremely complicated and gross approximations and numerous simplifications are necessary to allow the development of even the most rudimentary of models. A list of the major simplifications made in this model appears below:

(1) Shear and body forces are neglected (except for dryout prediction)

(2) The flow is assumed to be in thermal equilibrium with both phases at the saturation temperature.

(3) The annulus is assumed to be round and straight with a diameter equal to its hydraulic diameter

(4) The fluid properties (thermal conductivity, specific heat, and dynamic viscosity) of carbon dioxide are used in place of those of nitrous oxide.

(5) A numerical method with first order accuracy is used to predict dryout

(6) The Groeneveld correlation for steam-water mixtures is used to characterize the heat transfer coefficients of nitrous oxide mist flow.

(7) The copper ring temperature is assumed constant through its thickness and within each thermocouple section.

(8) The system is assumed to operate at steady state. 
(9) All heat transferred by the hot side at the copper annulus surface is assumed transferred to the coolant.

Based on these assumptions, it is likely that a close quantitative comparison will not be made between the model and experimental results. Nevertheless, valuable qualitative information has been gathered over the course of this study. First, the model developed is capable of predicting whether or not dryout and even superheat should occur in the annulus. It shows that is likely that the applied heat flux exceeds the critical heat flux in most Cal Poly firings. However, because the type of dryout is mist rather than inverted annular flow boiling, the drop in heat transfer coefficients is relatively mild and the copper temperature is not excessive. Second, it showed that the pressure drops experienced by the two-phase fluid are strikingly similar to those experienced by an ideal gas. The biggest improvement that can be made to the model presented is the development and inclusion of a heat transfer coefficient correlation. Upon gathering of additional data, one should be formulated. The Varone and Rohsenow model is ideal for modeling the heat transfer coefficients and fluid temperatures in the mist flow postdryout, pre-superheat region. Implementing this model in the place of a Dittus-Boelter based correlation would be far superior, but it is not trivial and beyond the scope of this work. A numerical integration technique much like that developed for the RevellinThome model would be necessary. However, if there is interest in providing quantitative predictions for heat transfer coefficients in the mist flow regime, this model should be used. 
On the experimental side, the equipment was verified with the ideal gas experiment providing confidence in all subsequent two-phase results. A flow meter was implemented and calibrated that improved the accuracy of the mass flow rate monitoring. Additional work should be completed to improve the accuracy of the pressure transducers. One suggestion for achieving this would be to use differential pressure transducers to monitor pressure drop through the annulus as opposed to pressure magnitude. Thermocouples should be implemented that have a range low enough to monitor the nitrous oxide at its saturation temperature in the annulus (approximately $15^{\circ} \mathrm{F}$ ).

While this thesis made considerable progress toward a full understanding of the twophase nitrous oxide coolant system, some questions still remain. One of the original topics of interest in this thesis was the possibility of nitrous oxide decomposition. This model does not directly address this issue. However, because it shows that superheating will not occur at standard operating conditions, it indirectly suggests that neither will decomposition (note that Rhodes showed that decomposition in the liquid phase was selfquenching (1)). Also, as the model includes the case in which the nitrous oxide does become superheated, it would be relatively simple to implement gaseous dissociation criteria in the future. More information about temperature dissociation criterion can be found in (24).

Two-phase choking was also of interest at the outset of this project. This issue is not addressed in the model developed previously. It is common knowledge within the twophase flow field that predicting such occurrences as choking is no simple task. While 
elementary models do exist that attempt to predict choking, none has been successful in accurately predicting such conditions (3). Most of these models depend on empirical correlations for predicting the bulk speed of sound of the two-phase mixture and are therefore limited to specific geometry and fluids. The complexity of the hybrid rocket cooling system makes it a poor choice for first application of such a model. If a twophase model were to be developed to predict choking, a much simpler apparatus should be built for preliminary testing (i.e. vertical round tube with unheated entrance length). Additional information about existing two-phase choked models can be found in (3). 


\section{BIBLIOGRAPHY}

1. Rhodes, G.W. Investigation of Decomposition Characteristics of Gaseous and Liquid Nitrous Oxide. Kirtland Air Force Base, New Mexico : Air Force Weaoins Laboratory, July 1974.

2. Zucrow, Maurice J. and Hoffman, Joe D. Gas Dynamics Volume I. New York : John Wiley and Sons, 1976.

3. Levy, Salomon. Two-Phase Flow in Complex Systems. New York: John Wiley and Sons, 1999.

4. Non-Boiling Heat Transfer in Gas-Liquid Flow in Pipes. Ghajar, A.J. 2005, Journal of the Barzilian Society of Mechanical Sciences and Engineering.

5. Carey, Van P. Liquid-Vapor Phase-Change Phenomena. Washington : Hemishphere Publishing Corporation, 1992.

6. Varone, Anthony F. and Rohsenow, Warren M. The Influence of the Dispersed Phase on the Convective Heat Transfer in Dispersed Flow Film Boiling. s.1.: Massachussetts Institute of Technology, 1990.

7. Chart Correlation for Saturated Boiling Heat Transfer: Equations and Further Study. Shah, M.M. 1980, pp. 185-196.

8. Correlation for Boiling Heat Transfer to Saturated Fluids in Convective Flow. Chen, John C. 1966, I\&EC Process Design and Development 5, pp. 322-329.

9. The 1995 Look-Up Table for Critical heat Flux in Tubes. Groeneveld, D.C., et al. 1996, Nuclear Engineering and Design 163, pp. 1-23. 
10. A Theoretical Model for the Prediction of the Critical Heat Flux in Heated Microchannels. Revellin, Remi and Thome, John R. 2008, International Journal of Heat and Mass Transfer 51, pp. 1216-1225.

11. Status of Prediction Methods for Critical Heat Fluxes in Mini and Microchannels. Revellin, Remi, Mishima, Kaichiro and Thome, John R. 2009, International Journal of Heat and Fluid Flow.

12. Cal Poly, Rolling Hills Research Corporation. A Reusable, Oxidizer-Cooled, Hybrid Aerospike Rocket Motor for Flight Test. 2009.

13. Bartz, D.R. A Simple Equation for Rapid Estimation of Rocket Nozzle Convective Heat Transfer Coefficients. Jet Propulsion. January 1957, pp. 49-51.

14. Incropera, Frank P. and DeWitt, David P. Introduction to Heat Transfer. s.l. : John Wiley and Sons, 2007.

15. Penner, S.S. Equilibrium Radiation Properties of Gases. [book auth.] James P. (editor) Rohsenow and James P. (editor) Hartnett. Handbook of Heat Transfer. New York : McGraw-Hill, 1973, pp. 15.72-15.93.

16. Jiji, Latif M. Heat Convection. The Netherlands : Springer, 2006.

17. Brennan, Peter. Simulation of an Oxidizer-Cooled Hybrid Rocket Throat: Methodology Validation for Design of a Cooled Aerospike Nozzle. Master's Thesis. s.l. : California Polytechnic State University, 2009.

18. Thermophysical Properties Research Center, Purdue University. Volume 6: Specific Heat: Nonmetallic Liquids and Gases. Thermophysical Properties of Matter. New York - Washington : IFI/Plenum, 1970, pp. 94-95. 
19. U.S. Secretary of Commerce. National Institute of Standards and Technology. Thermophysical Properties of Fluid Systems. [Online] 2008. http://webbook.nist.gov/chemistry/fluid/.

20. CINDAS LLC. Thermophysical Properties of Matter Database. [Software] West Lafayette : s.n., 2009.

21. On the Similarity between Carbon Dioxide and Nitrous Oxide. Rankine, A.O. s.1. : The Royal Society, 1921. Proceedings of the Royal Society of London. Series A, Containing Papers of a Mathematical and Physical Character. pp. 369-374.

22. Tong, L.S. and Tang, Y.S. Boiling Heat Transfer and Two-Phase Flow. Washington, D.C. : Taylor and Francis, 1997.

23. Novak, Vladimir. Experimental and Numerical Studies of Mist Cooling with Thin Evaporating Subcooled Liquid Films. s.1. : Georgia Institute of Technology, May 2006.

24. Air Products and Chemicals, Inc. Safetygram \#20: Nitrous Oxide. Air Products. [Online] 2008. http://www.airproducts.com.

25. Flow Boiling Heat Transfer to Carbon Dioxide: Gemeral Prediction Method. Thome, John R. and Hajal, Jean El. 2004, International Journal of Refrigeration 27, pp. 294301.

26. Post-Dryout Heat Transfer Characteristics in Horizontal Mini-Tubes and a Prediction Method for Flow Boiling of CO2. Yun, R. and Kim, Y. 2009, International Journal of Refrigeration 32, pp. 1085-1091.

27. [book auth.] P. Balachandran. Fundamentals of Compressible Fluid Dynamics. New Dehli : Prentice-Hall of India, 2006, pp. 183-216. 
28. Karabeyoglu, Arif, et al. Modeling of N2O Decomposition Events. Sunnyvale, CA : Space Propulsion Group Inc.

29. Liles, Dennis R. Two Phase Flow. Los Alamos : Los Alamos National Laboratory, 1997.

30. Balasubramaniam, R., et al. Two Phase Flow Modeling:Summary of Flow Regimes and Pressure Drop Correlations in Reduced and Partial Gravicty. Washington D.C. : National Aeronautics and Space Administration, 2006.

31. Penner, S.S. Equilibrium Properties of Gases. [book auth.] Warren M. Rohsenow and James P. Hartnett. Handbook of Heat Transfer. s.l. : McGraw Hill Book Company, 1973, pp. 15.62-15.81.

32. A Model for Predicting Flow Regime Transitions in Horizontal and Near Horizontal Gas-Liquid Flow. Taitel, Yemada and Dukler, A.E. January, 1976, AlChE Journal, pp. 47-55. 


\title{
APPENDIX A: HELIUM TEST MATLAB CODE(S)
}

\author{
\%IDEAL GAS RAYLEIGH FLOW SIMULATION\% \\ $\%$ Calculates fluid property changes due to heat addiitons for a perfect gas \\ \%in coolant annulus. To run, updated the "INPUTS" section with the \\ \%appropriate values for the specific test of interest. \\ $\% \%$ VARIABLES \\ \% T_in: Annulus inlet temperature $(\operatorname{deg} \mathrm{R})$ \\ $\%$ P_in: Annulus inlet pressure (psia) \\ \%mdot: $\quad$ Mass flow rate $(\mathrm{lbm} / \mathrm{sec})$ \\ \%A_cold: Surface area of copper exposed to coolant (in^2) \\ $\%$ A: $\quad$ Cross-sectional area of coolant flow path (in^2) \\ $\%$ P: $\quad$ Perimeter of coolant flow path (in) \\ $\%$ L: $\quad$ Length of coolant flow path (in) \\ $\% \mathrm{Cp}: \quad$ Specific heat at constant pressure of coolant (BTU/lbm*degR) \\ $\% \mathrm{G}: \quad$ Ratio of specific heatrs of coolant (--) \\ \%Rbar: $\quad$ Specific gas constant of coolant (BTU/lbm*degR) \\ \%mu: $\quad$ Dynamic viscosity of coolant $(\mathrm{lbm} / \mathrm{ft} * \mathrm{sec})$ \\ \%k_fluid: Thermal conductivity of coolant (BTU/sec*in*degR) \\ \%h_hot: Hot side heat transfer coeffcient as determined by Bartz for \\ $\%$ given $\mathrm{O} / \mathrm{F}$ and chamber pressure (BTU/in^ ${ }^{\wedge} * \sec ^{*}$ degR) \\ \%A_hot: Surface area of copper exposed to combustion gases (in^2) \\ \%Tgh: Combustion gas temperature at nozzle throat from TEP (deg R) \\ $\% \mathrm{~T} \_\mathrm{Cu}$ _guess:Approximate copper temperature (deg R) \\ $\%$ Dh: Coolant annulus hydraulic diameter (in) \\ \%Gflow: Mass flux (lbm/in^2*sec) \\ \%Pr: $\quad$ Prandtl number (--) \\ \%Re: Reynolds number (--) \\ $\%$ Cf: $\quad$ Friction factor (--) \\ $\%$ St: $\quad$ Stanton number (--) \\ $\% \mathrm{Nu}: \quad$ Nusselt number (--) \\ $\%$ h_cold: Coolant heat transfer coefficient (BTU/in^2*sec*degR) \\ \%rho_in: Annulus inlet density $\left(\mathrm{lbm} / \mathrm{ft}^{\wedge} 3\right)$ \\ $\%$ u_in: Annulus inlet velocity $(\mathrm{ft} / \mathrm{sec})$ \\ \%c_in: Annulus inlet speed of sound $(\mathrm{ft} / \mathrm{sec})$ \\ \%Min: Annulus inlet Mach number (--) \\ \%T_o_in: Annulus inlet stagnation temperature (deg R) \\ $\% \mathrm{M}: \quad$ Vector of possible Mach numbers between inlet and sonic(--) \\ \%x: $\quad$ Annulus axial location (in) \\ \%Qtu2: Conversion factor from $\mathrm{ft}^{\wedge} 2 / \mathrm{sec}^{\wedge} 2$ to BTU/lbm \\ $\%$ Btf: $\quad$ Conversion factor from $\mathrm{ft}^{*} \mathrm{lbf}$ to BTU \\ $\%$ i2tf2: Conversion factor from in^2 to $\mathrm{ft}^{\wedge} 2$ \\ \%y: $\quad$ Loop variable \\ \%q: $\quad$ Heat transfer rate (BTU/sec); \\ \%q_new: Heat transfer rate calculated with updated copper
}




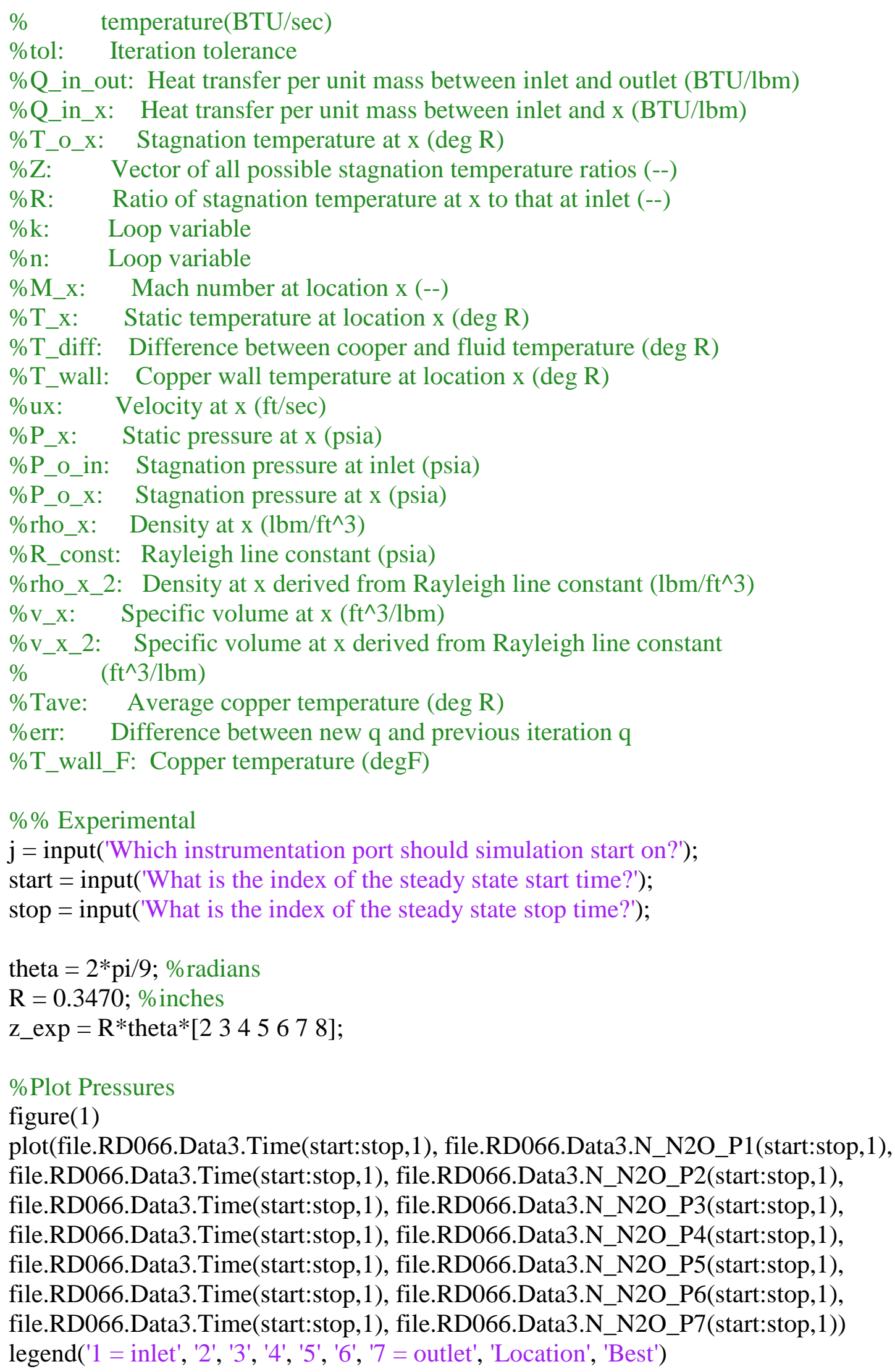


xlabel('Time (sec)')

ylabel('Annulus Pressure (psig)')

\%Average pressures

P1 = mean(file.RD066.Data3.N_N2O_P1(start:stop,1));

P2 = mean(file.RD066.Data3.N_N2O_P2(start:stop,1));

P3 = mean(file.RD066.Data3.N_N2O_P3(start:stop,1));

P4 = mean(file.RD066.Data3.N_N2O_P4(start:stop,1));

P5 = mean(file.RD066.Data3.N_N2O_P5(start:stop,1));

P6 = mean(file.RD066.Data3.N_N2O_P6(start:stop,1));

P7 = mean(file.RD066.Data3.N_N2O_P7(start:stop,1));

P_exp $=$ [P1 P2 P3 P4 P5 P6 P7];

delta_P_exp $=$ P_exp(j)*ones $\left(1\right.$, length $\left.\left(P \_\exp \right)\right)$ - $P \_$exp;

$\%$ figure(5)

$\%$ plot(z_exp, P_exp, 'kd')

$\%$ xlabel('Distance Through Annulus (inches)')

$\%$ ylabel('Coolant Pressure (psig)')

$\%$ Correct Copper Temperatures

T_Cu1 $=0.0008 *\left(f i l e . R D 066 . D a t a 2 . C T \_T 8\right) . \wedge 2+1.4254 *$ file.RD066.Data2.CT_T8 27.739;

T_Cu2 $=0.0008 *\left(f i l e . R D 066 . D a t a 2 . C T \_T 9\right) . \wedge 2+1.4254 *$ file.RD066.Data2.CT_T9 27.739;

T_Cu3 $=0.0008 *\left(f i l e . R D 066 . D a t a 2 . C T \_T 10\right) . \wedge 2+1.4254 *$ file.RD066.Data2.CT_T10 27.739;

T_Cu4 $=0.0008 *\left(f i l e . R D 066 . D a t a 2 . C T \_T 11\right) . \wedge 2+1.4254 *$ file.RD066.Data2.CT_T11 27.739;

T_Cu5 $=0.0008 *\left(f i l e . R D 066 . D a t a 2 . C T \_T 12\right) . \wedge 2+1.4254 *$ file.RD066.Data2.CT_T12 27.739;

T_Cu6 $=0.0008 *\left(f i l e . R D 066 . D a t a 2 . C T \_T 13\right) . \wedge 2+1.4254 *$ file.RD066.Data2.CT_T13 27.739;

T_Cu7 $=0.0008 *\left(f i l e . R D 066 . D a t a 2 . C T \_T 14\right) . \wedge 2+1.4254 *$ file.RD066.Data2.CT_T14 27.739;

\%Plot Copper Temperatures

figure(2)

plot(file.RD066.Data2.Time(start:stop,1), T_Cu1(start:stop,1),

file.RD066.Data2.Time(start:stop,1),T_Cu2(start:stop,1),

file.RD066.Data2.Time(start:stop,1), T_Cu3(start:stop,1),

file.RD066.Data2.Time(start:stop,1), T_Cu4(start:stop,1),

file.RD066.Data2.Time(start:stop,1), T_Cu5(start:stop,1),

file.RD066.Data2.Time(start:stop,1), T_Cu6(start:stop,1),

file.RD066.Data2.Time(start:stop,1), T_Cu7(start:stop,1))

legend('1 = inlet', '2', '3', '4', '5', '6', '7 = outlet', 'Location', 'Best')

xlabel('Time (sec)') 


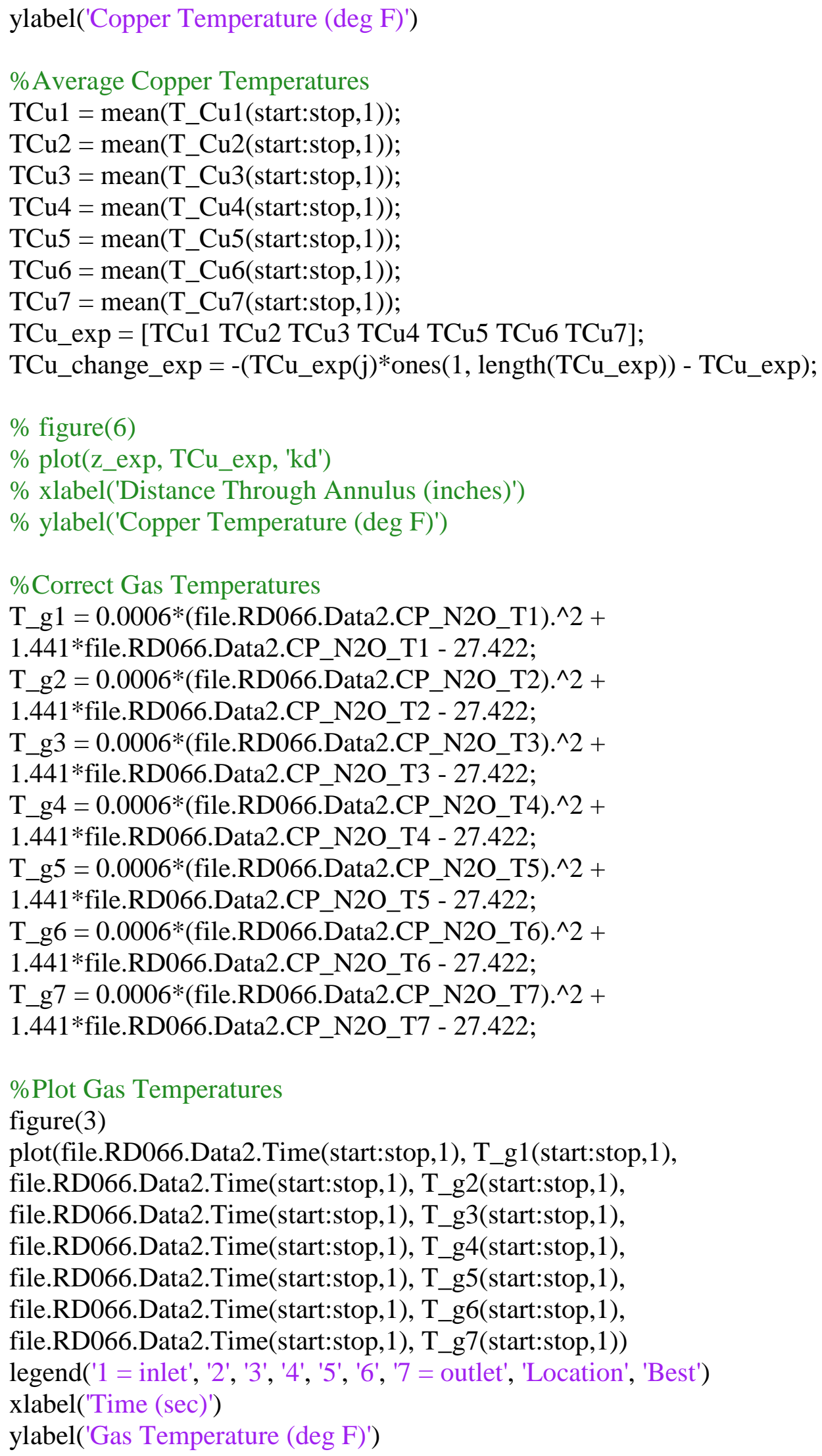




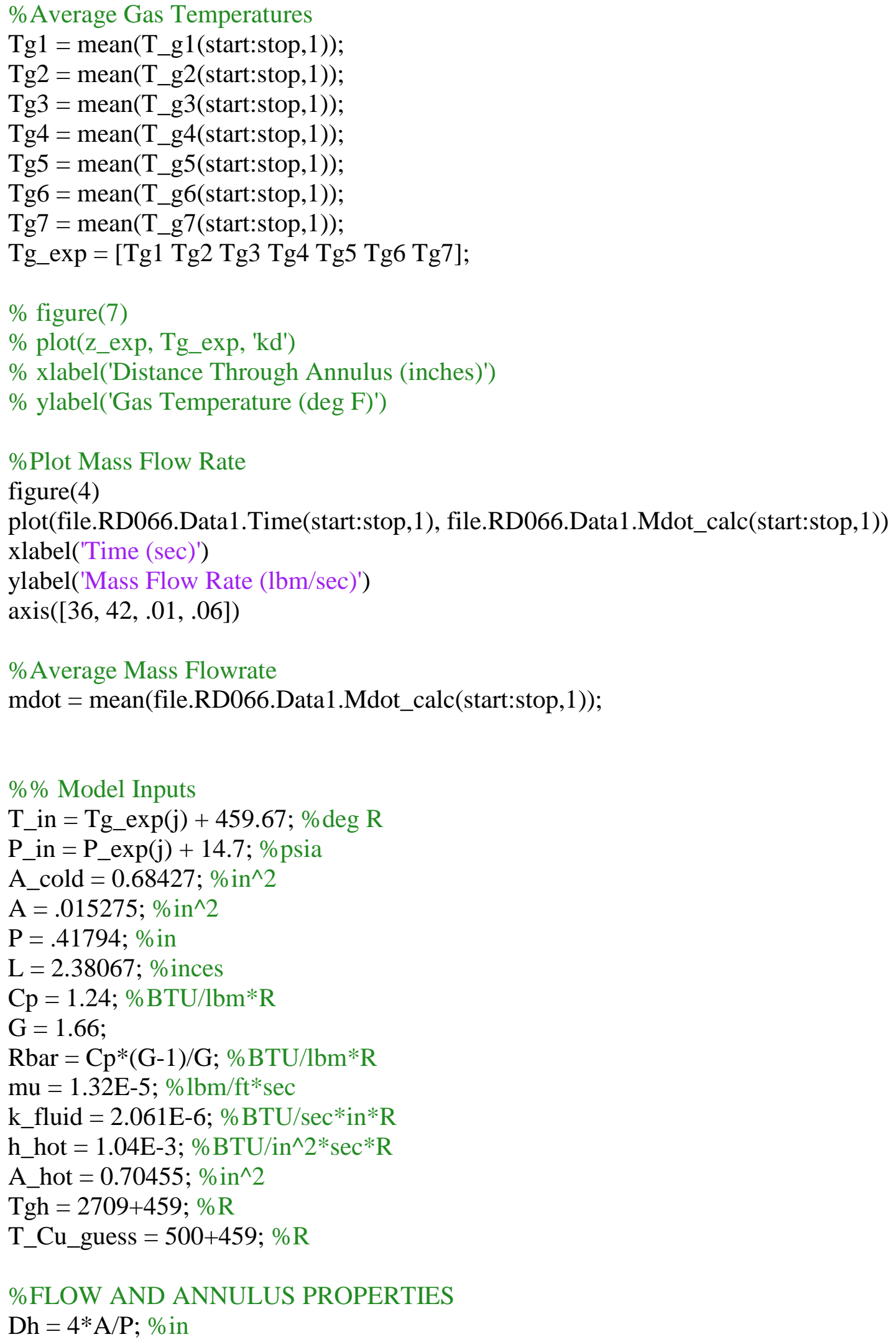




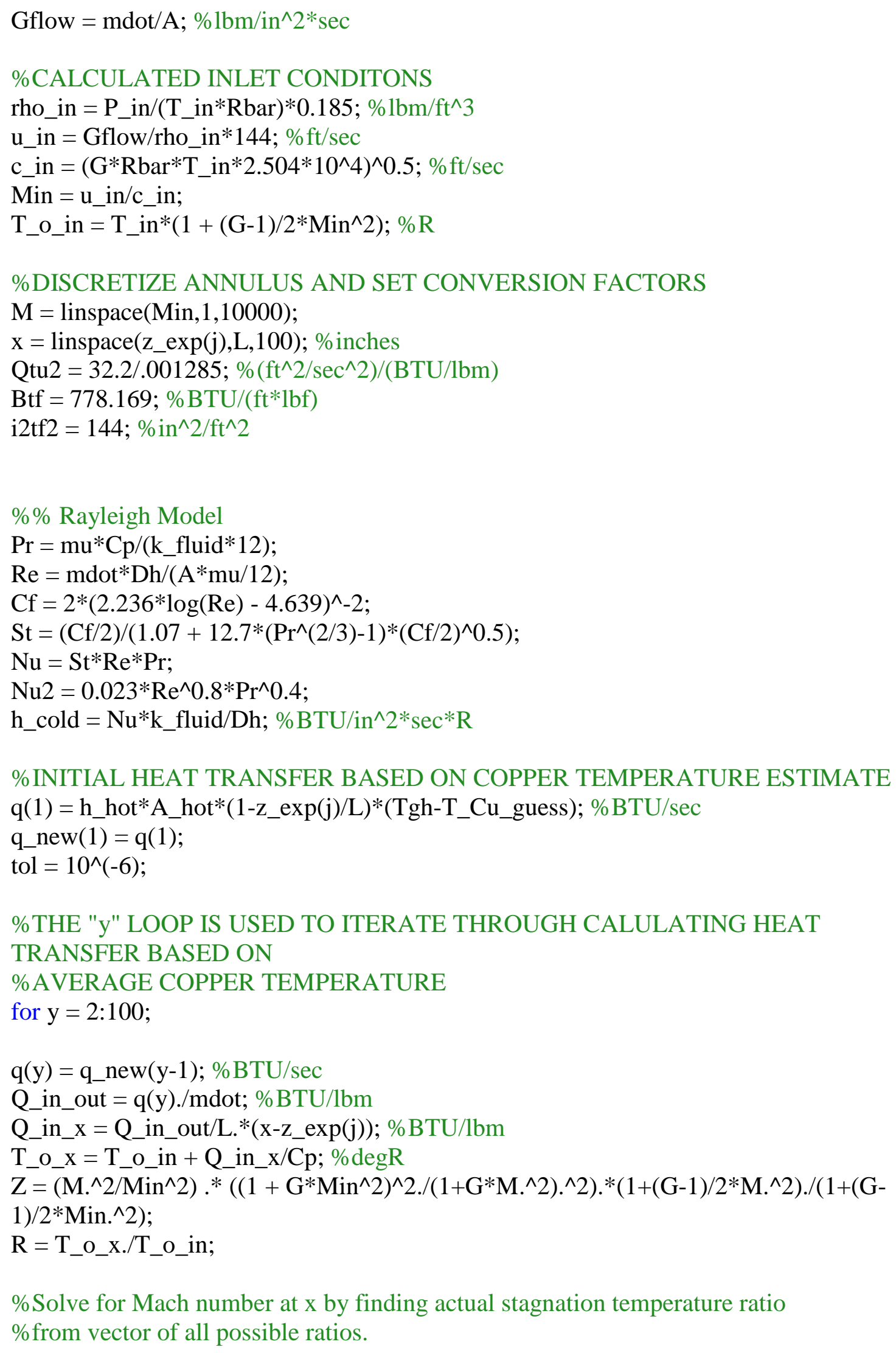




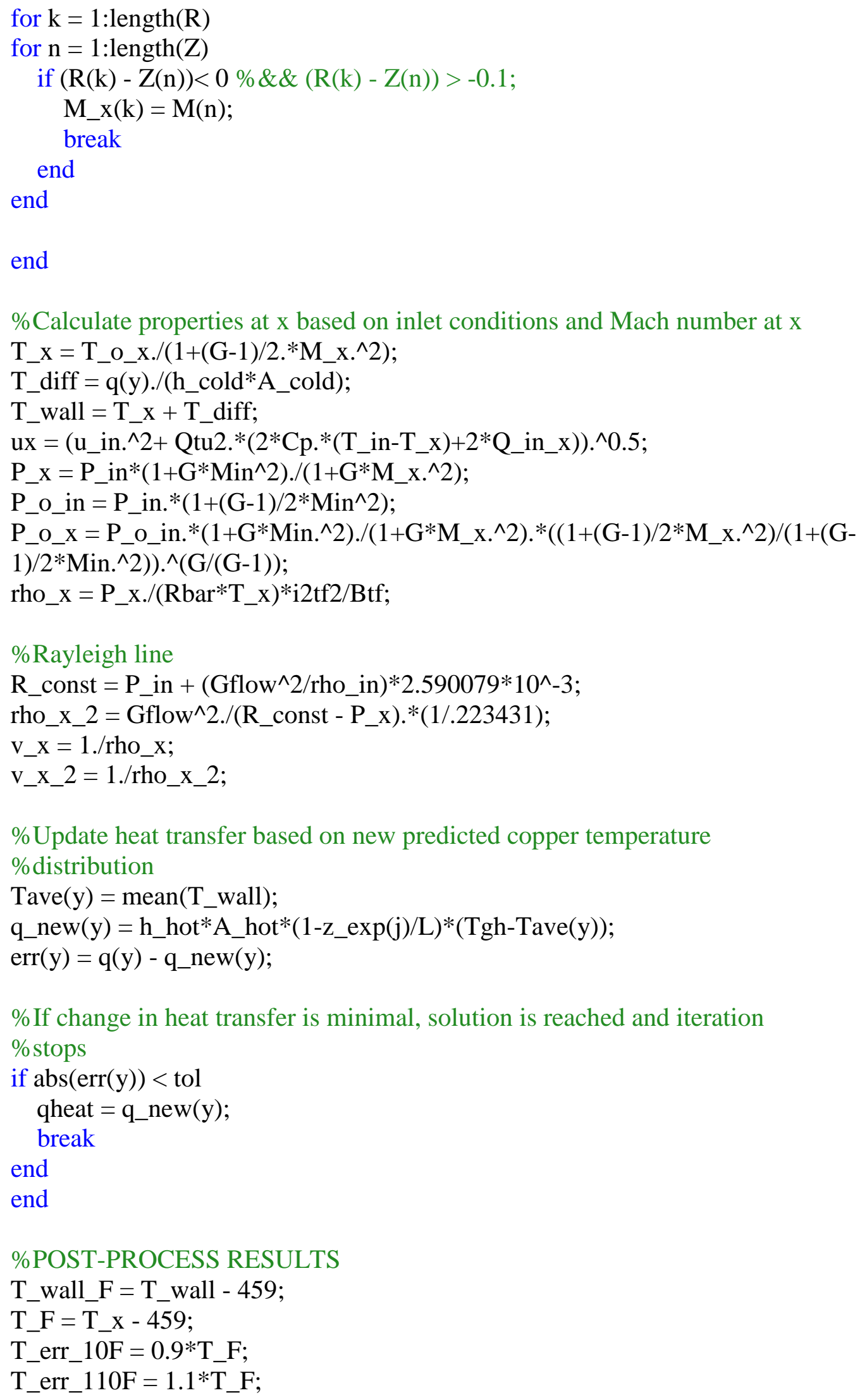




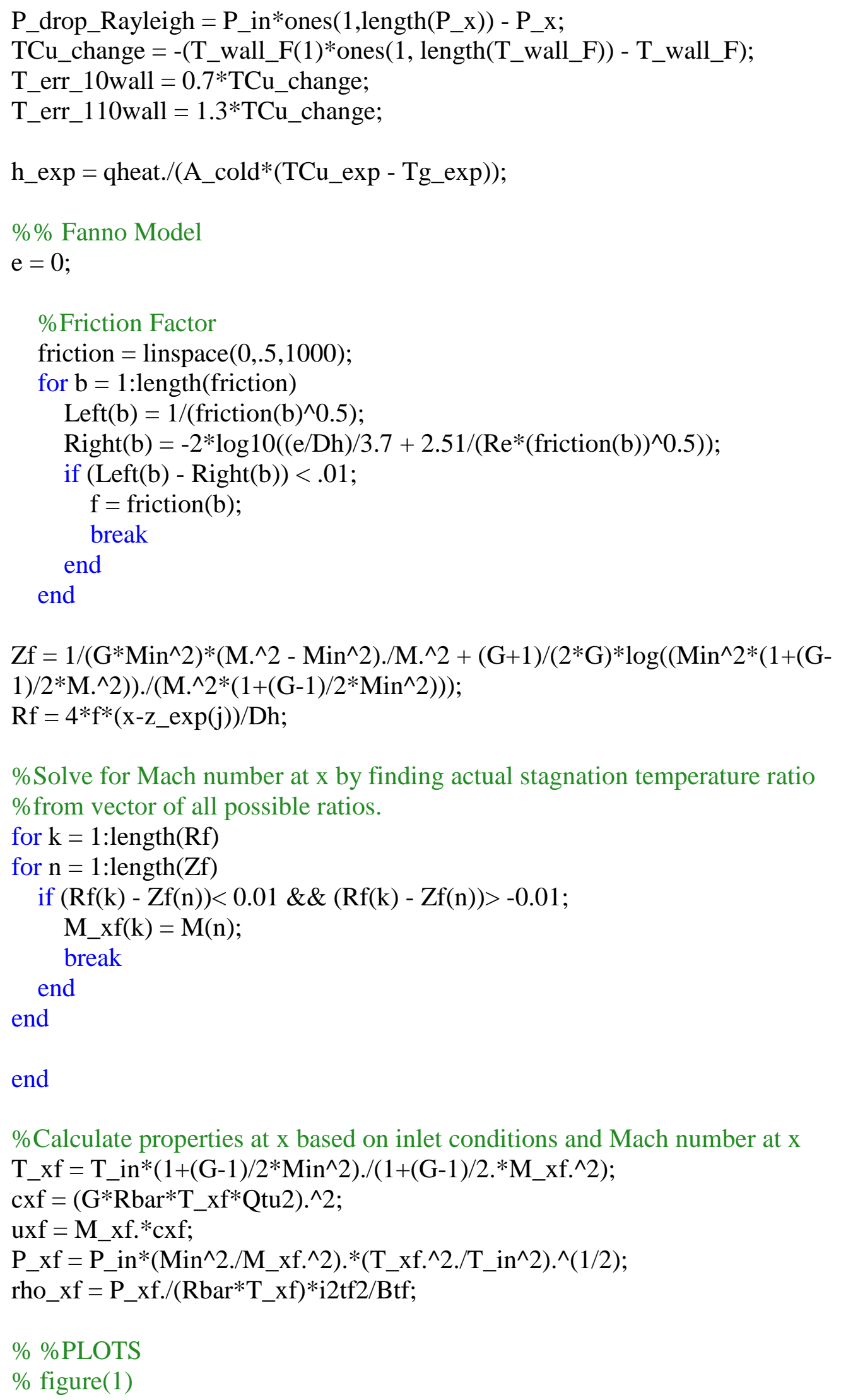




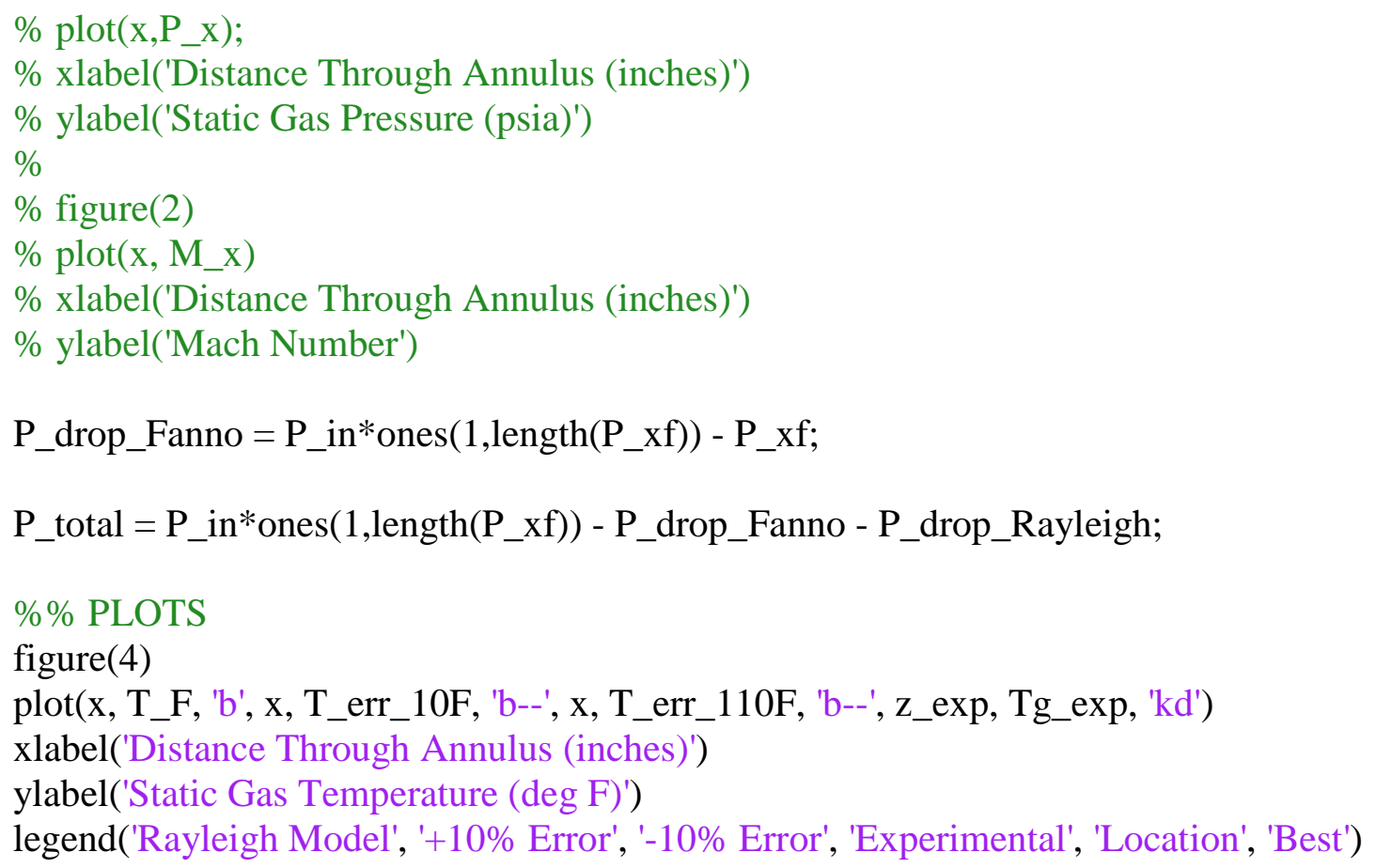




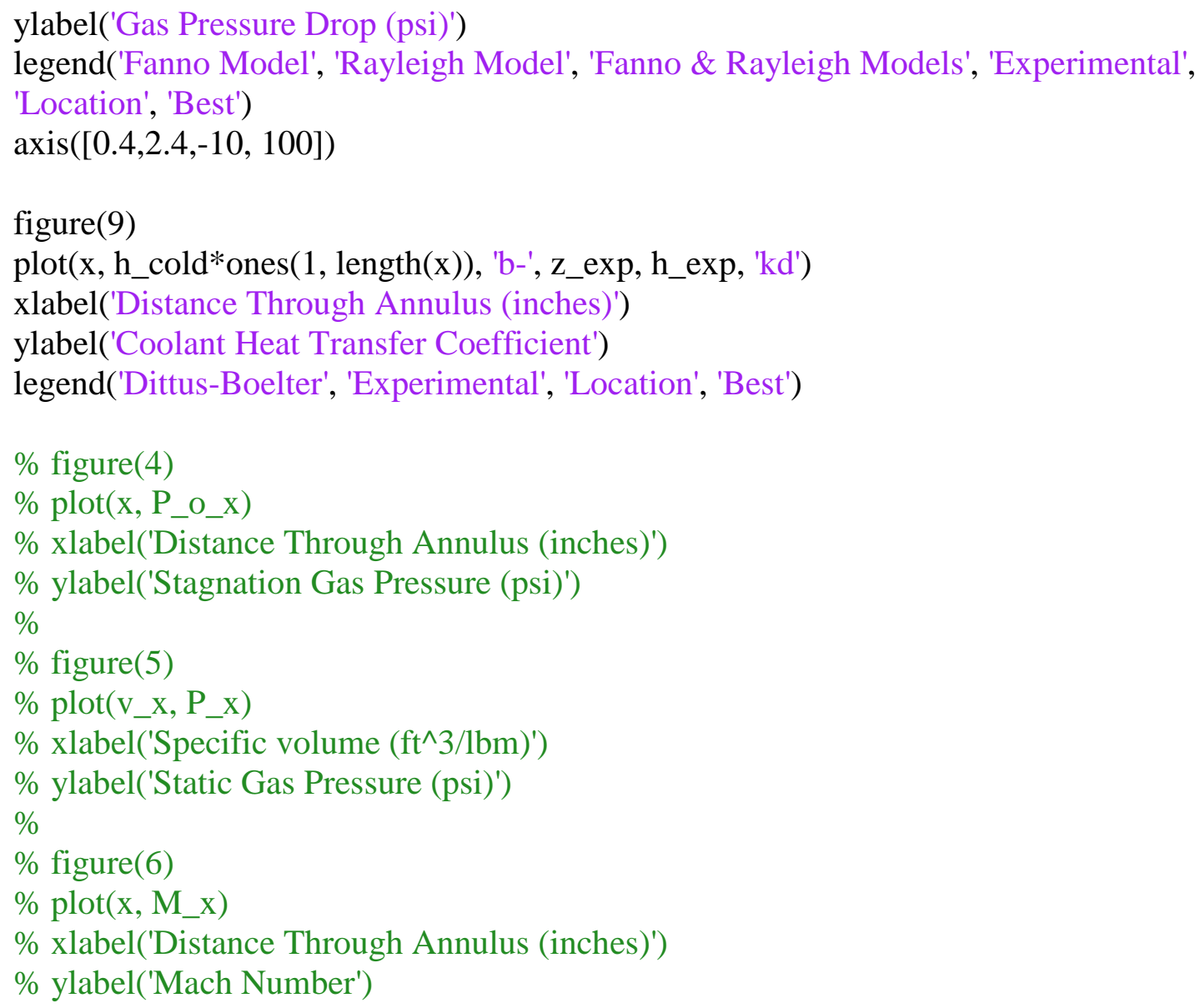




\section{APPENDIX B: NITROUS OXIDE MATLAB CODE(S)}

$\% \% \% \% \% \% \% \% \% \% \% \% \% \% \% \% \% \% \% \% \% \% \% \%$ HOMOGENEOUS FLOW MODEL $\% \% \% \% \% \% \% \% \% \% \% \% \% \% \% \% \% \% \% \%$

$\%$ Implements one-dimensional model to two-phase rayleigh flow of nitrous \%oxide. To run, update "INPUTS" section to run conditions. Save Excel file $\%$ to computer on which the program is being run and update file paths in the $\% " R E A D$ IN" section below.

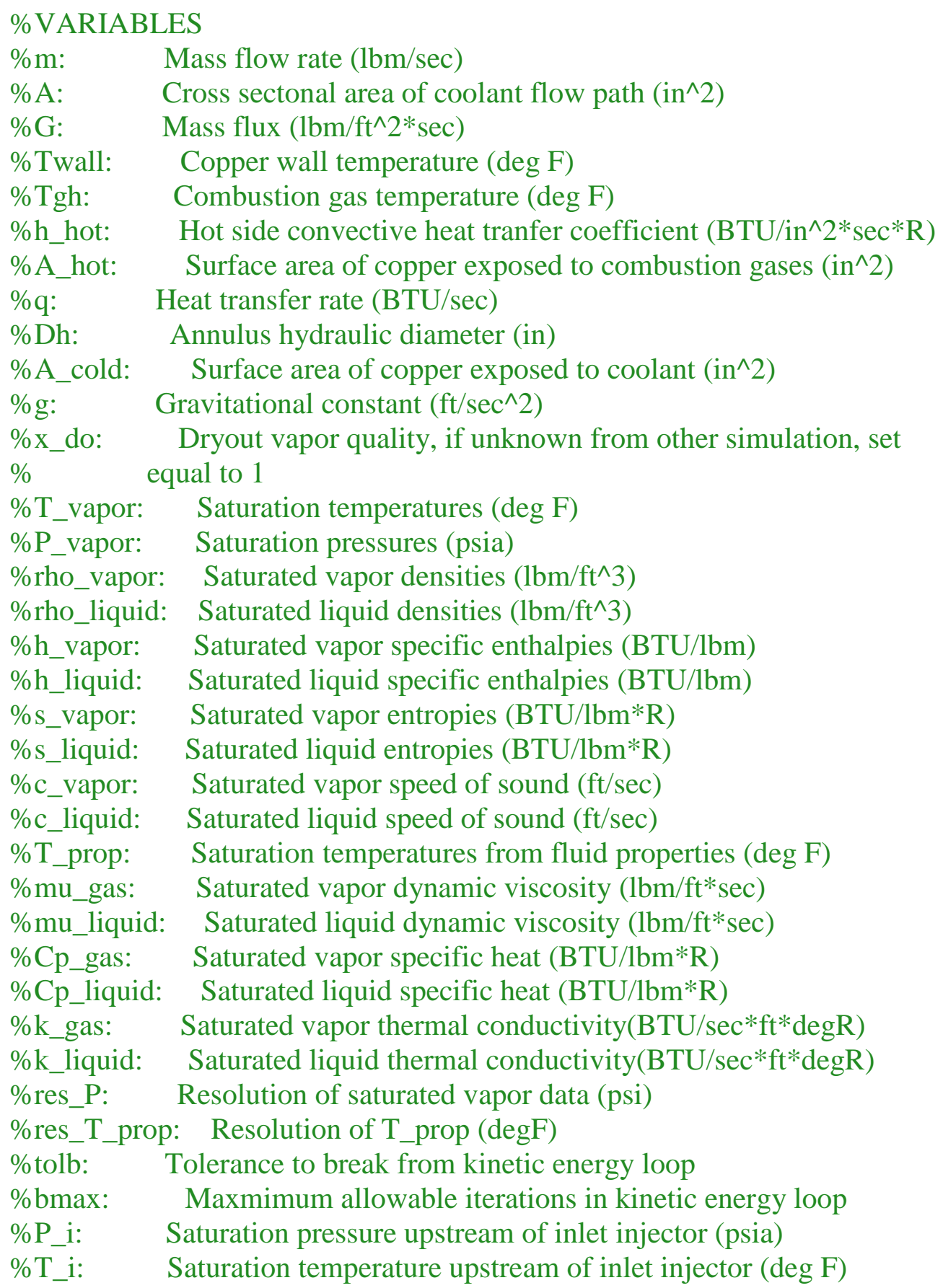




\begin{tabular}{|c|c|}
\hline & $\begin{array}{l}\text { Saturated liquid enthalpy upstream of inlet injector } \\
\text { (BTU/lbm) }\end{array}$ \\
\hline$\% \mathrm{P}$ in: & Annulus inlet pressure (psia) \\
\hline th_l: & Saturated liquid enthalpy at inlet pressure (BTU/lbm) \\
\hline oh_g: & Saturated vapor enthalpy at inlet pressure (BTU/lbm) \\
\hline$\%$ s_1: & Saturated liquid entropy at inlet pressure (BTU/lbm) \\
\hline 7os_g: & Saturated vapor entropy at inlet pressure (BTU/lbm) \\
\hline \%c_l: & Saturated liquid speed of sound at inlet pressure $(\mathrm{ft} / \mathrm{sec})$ \\
\hline c_g: & Saturated vapor speed of sound at inlet pressure $(\mathrm{ft} / \mathrm{sec})$ \\
\hline \%orho_1: & Saturated liquid density at inlet pressure $\left(\mathrm{lbm} / \mathrm{ft}^{\wedge} 3\right)$ \\
\hline orho_g: & Saturated vapor density at inlet pressure $\left(\mathrm{lbm} / \mathrm{ft}^{\wedge} 3\right)$ \\
\hline 7oh: & Mixture enthalpy (BTU/lbm) \\
\hline 7ox: & Mixture quality (--) \\
\hline brho: & Mixture density $\left(\mathrm{lbm} / \mathrm{ft}^{\wedge} 3\right)$ \\
\hline \%bias: & Number of solution locations axially through annulus \\
\hline bL: & Length of annulus (in) \\
\hline 7odz: & Length of discretized section \\
\hline odq: & Heat transfer rate in single discretized section (BTU/sec) \\
\hline$\%$ \%: & Mixture velocity $(\mathrm{ft} / \mathrm{sec})$ \\
\hline$\% \mathrm{P}:$ & Mixture pressure (psia) \\
\hline$\% \mathrm{~T}:$ & Mixture temperature $(\operatorname{deg} F)$ \\
\hline$\% \mathrm{v}:$ & Mixture specific volume $\left(\mathrm{ft}^{\wedge} 3 / \mathrm{lbm}\right)$ \\
\hline \%alpha: & Void fraction \\
\hline \%loc_T: & Index of temperature in saturation temperature vector (--) \\
\hline$\%$ mu_l: & Saturated liquid viscosity at temperature $\left(\mathrm{lbm} / \mathrm{ft}^{*} \mathrm{sec}\right)$ \\
\hline \%mu_g: & Saturated vapor viscosity at temperature(lbm/ft*sec) \\
\hline$\%$ k_l: & Saturated liquid conductivity at temperature $(\mathrm{BTU} / \mathrm{sec} * \mathrm{ft} * \mathrm{R})$ \\
\hline \%k_g: & Saturated vapor conductivity at temperature $(\mathrm{BTU} / \mathrm{sec} * \mathrm{ft} * \mathrm{R})$ \\
\hline 7ocp_l: & Saturated liquid specific heat at temperature(BTU/lbm*R) \\
\hline$\%$ Cp_g: & Saturated vapor specific heat at temperature(BTU/lbm*R) \\
\hline$\% \mathrm{k}:$ & Mixture conductivity at temperature(BTU/sec*ft*R) \\
\hline \%mu: & Mixture viscosity at temperature $(\mathrm{lbm} / \mathrm{ft} * \mathrm{sec})$ \\
\hline$\% \mathrm{Cp}:$ & Mixture specific heat at temperature(BTU/lbm*R) \\
\hline$\% \operatorname{Pr}:$ & Mixture Prandtl number (--) \\
\hline$\% \mathrm{Re}:$ & Mixture Reynolds number (--) \\
\hline$\% \mathrm{Nu}:$ & Mixture Nusselt number \\
\hline$\%$ h_TP: & Mixture convective heat transfer coefficient \\
\hline & $(\mathrm{BTU} / \mathrm{in} \wedge 2 * \mathrm{sec})$ \\
\hline \%Re_g: & Vapor Reynolds number (--) \\
\hline$\% \operatorname{Re} \_1:$ & Liquid Reynolds number (--) \\
\hline$\%$ Pr_l: & Liquid Prandtl number (--) \\
\hline$\%$ Во: & Boiling number (--) \\
\hline$\%$ Fr: & Froude number (--) \\
\hline$\% \mathrm{Co}:$ & Convection number (--) \\
\hline \%h_liquid: & Liquid convective heat transfer coefficient(BTU/in^2*sec) \\
\hline oh_nb: & Two-phase nucleate boiling heat transfer \\
\hline
\end{tabular}




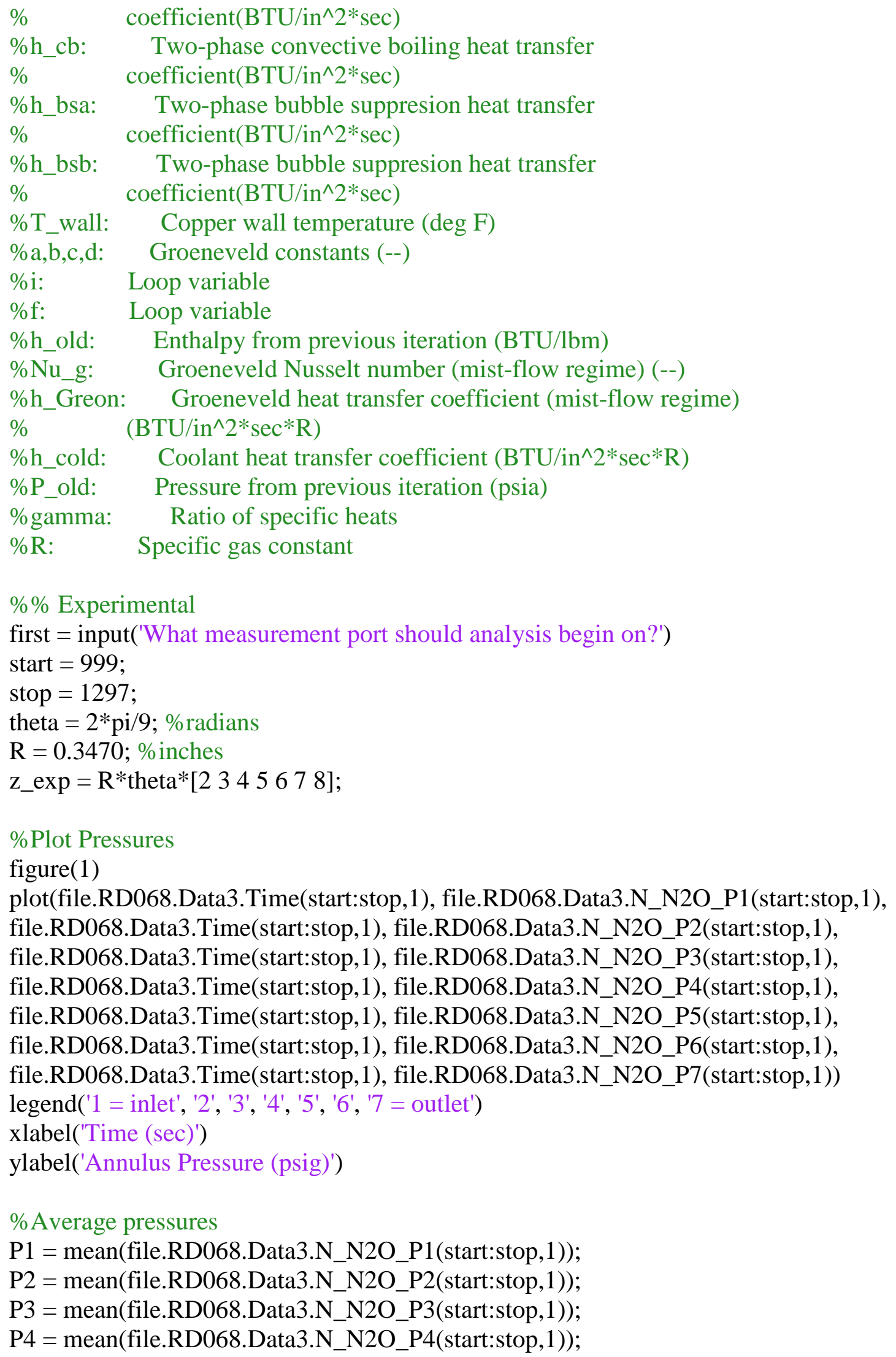




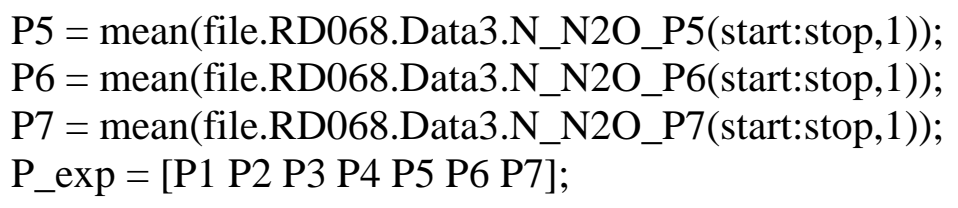

figure(2)

errorbar(z_exp, P_exp, linspace(2.5, 2.5, length(P_exp)), 'kd'); xlabel('Distance Through Annulus (inches)')

ylabel('Coolant Pressure (psig)')

hold on

\section{\%Plot Copper Temperatures}

figure(3)

plot(file.RD068.Data2.Time(start:stop,1), file.RD068.Data2.CT_T8(start:stop,1), file.RD068.Data2.Time(start:stop,1), file.RD068.Data2.CT_T9(start:stop,1), file.RD068.Data2.Time(start:stop,1), file.RD068.Data2.CT_T10(start:stop,1), file.RD068.Data2.Time(start:stop,1), file.RD068.Data2.CT_T11(start:stop,1), file.RD068.Data2.Time(start:stop,1), file.RD068.Data2.CT_T12(start:stop,1), file.RD068.Data2.Time(start:stop,1), file.RD068.Data2.CT_T13(start:stop,1), file.RD068.Data2.Time(start:stop,1), file.RD068.Data2.CT_T14(start:stop,1)) legend('1 = inlet', '2', '3', '4', '5', '6', '7 = outlet') xlabel('Time (sec)') ylabel('Copper Temperature (deg F)')

\section{\%Average Copper Temperatures}

$\mathrm{TCu} 1=$ mean(file.RD068.Data2.CT_T8(start:stop,1));

$\mathrm{TCu} 2=$ mean(file.RD068.Data2.CT_T9(start:stop,1));

TCu3 = mean(file.RD068.Data2.CT_T10 (start:stop,1));

TCu4 = mean(file.RD068.Data2.CT_T11(start:stop,1));

TCu5 = mean(file.RD068.Data2.CT_T12(start:stop,1));

TCu6 = mean(file.RD068.Data2.CT_T13(start:stop,1));

TCu7 = mean(file.RD068.Data2.CT_T14(start:stop,1));

TCu_exp $=$ [TCu1 TCu2 TCu3 TCu4 TCu5 TCu6 TCu7];

figure(4)

errorbar(z_exp, TCu_exp, linspace(3, 3, length(TCu_exp)), 'kd') xlabel('Distance Through Annulus (inches)') ylabel('Copper Temperature (deg F)')

hold on

\section{$\%$ Plot Gas Temperatures}

figure(5)

plot(file.RD068.Data2.Time(start:stop,1), file.RD068.Data2.CP_N2O_T1(start:stop,1), file.RD068.Data2.Time(start:stop,1), file.RD068.Data2.CP_N2O_T2(start:stop,1), file.RD068.Data2.Time(start:stop,1), file.RD068.Data2.CP_N2O_T3(start:stop,1), file.RD068.Data2.Time(start:stop,1), file.RD068.Data2.CP_N2O_T4(start:stop,1), 
file.RD068.Data2.Time(start:stop,1), file.RD068.Data2.CP_N2O_T5(start:stop,1), file.RD068.Data2.Time(start:stop,1), file.RD068.Data2.CP_N2O_T6(start:stop,1), file.RD068.Data2.Time(start:stop,1), file.RD068.Data2.CP_N2O_T7(start:stop,1)) legend('1 = inlet', '2', '3', '4', '5', '6', '7 = outlet') xlabel('Time (sec)')

ylabel('Gas Temperature (deg F)')

\%Average Copper Temperatures

$\mathrm{Tg} 1=$ mean(file.RD068.Data2.CP_N2O_T1(start:stop,1));

$\mathrm{Tg} 2=$ mean(file.RD068.Data2.CP_N2O_T2(start:stop,1));

Tg3 = mean(file.RD068.Data2.CP_N2O_T3(start:stop,1));

Tg4 = mean(file.RD068.Data2.CP_N2O_T4(start:stop,1));

$\mathrm{Tg} 5=$ mean(file.RD068.Data2.CP_N2O_T5(start:stop,1));

$\mathrm{Tg} 6=$ mean(file.RD068.Data2.CP_N2O_T6(start:stop,1));

Tg7 = mean(file.RD068.Data2.CP_N2O_T7(start:stop,1));

Tg_exp = [Tg1 Tg2 Tg3 Tg4 Tg5 Tg6 Tg7];

figure(6)

errorbar(z_exp, Tg_exp, linspace( 3 , 3, length(Tg_exp)), 'kd')

hold on

plot(z_exp, 36*ones(length(z_exp),1), 'k--')

xlabel('Distance Through Annulus (inches)')

ylabel('Gas Temperature (deg F)')

legend('Experimental Values', 'Measurement Minimum')

figure(7)

plot(file.RD068.Data1.Time(start:stop,1),

file.RD068.Data1.Cooling_Weight(start:stop,1))

mscale $=$ polyfit(file.RD068.Data1.Time(start:stop,1),

file.RD068.Data1.Cooling_Weight(start:stop,1), 1);

mscale_line $=$ mscale $(1) *$ file.RD068.Data1.Time $($ start:stop, 1$)+$ mscale $(2)$;

hold on

plot(file.RD068.Data1.Time(start:stop,1), mscale_line, 'k')

legend('Measured', 'Trendline')

\%Plot Mass Flow Rate

figure(8)

plot(file.RD068.Data1.Time(start:stop,1), file.RD068.Data1.Mdot_calc(start:stop,1))

xlabel('Time (sec)')

ylabel('Mass Flow Rate $(1 \mathrm{bm} / \mathrm{sec})$ ')

hold on

plot(file.RD068.Data1.Time(start:stop,1), -

mscale(1)*ones(length(file.RD068.Data1.Time(start:stop,1)),1), 'k')

legend('Flowmeter', 'Scale')

\%Average Mass Flowrate 
mdot $=$ mean $($ file.RD068.Data1.Mdot_calc(start:stop,1));

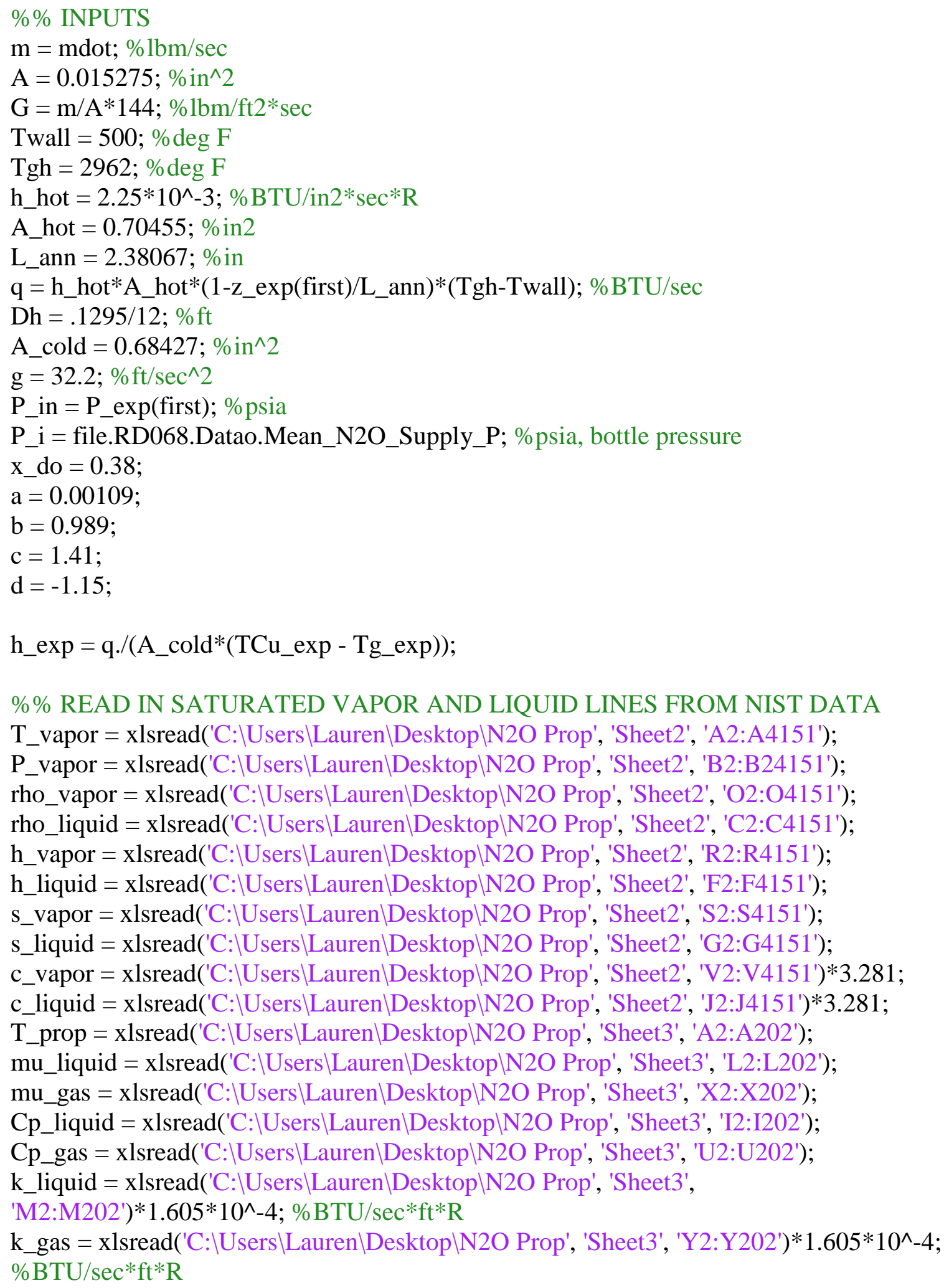


\%CALCULATE THE RESOLUTION OF INPUT DATA

res_P $=$ abs $\left(\mathrm{P}_{-}\right.$vapor(2) - P_vapor(1)); \%psia

res_T_prop $=$ abs(T_prop $\left.(2)-T \_p r o p(1)\right) ; \% \operatorname{degF}$

$\%$ SET TOLERANCE AND MAXIMUM NUMBER OF ITERATIONS ON KINETIC
ENERGY LOOP
tolb = 0.0001
bmax $=1000$

\%FIND BOTTLE CONDITIONS ASSUMING SATURATED LIQUID

T_i $=$ T_vapor $\left(\operatorname{round}\left(\left(\mathrm{P} \_\mathrm{i}-\mathrm{P} \_\right.\right.\right.$vapor $\left.\left.\left.(1)\right) / \mathrm{res}_{-} \mathrm{P}+1\right)\right) ; \% \mathrm{deg} \mathrm{F}$

$\mathrm{h} \_\mathrm{i}=\mathrm{h} \_$liquid(round $\left(\left(\mathrm{P} \_\mathrm{i}-\mathrm{P} \_\right.\right.$vapor(1))/res_P+1)$)$; \%BTU/lbm

\% INITIALIZE PRESSURE AND DENSITY VALUES FOR ITERATION

$\mathrm{h} \_1=\mathrm{h} \_$liquid(round $\left(\left(\mathrm{P} \_\right.\right.$in $-\mathrm{P} \_$vapor(1))/res_P $\left.)+1\right) ; \% \mathrm{BTU} / \mathrm{lbm}$

$\mathrm{h} \_\mathrm{g}=\mathrm{h} \_$vapor$\left(\operatorname{round}\left(\left(\mathrm{P} \_\right.\right.\right.$in-P_vapor(1))/res_P $\left.)+1\right) ; \% \mathrm{BTU} / \mathrm{lbm}$

$\mathrm{s} \_1=\mathrm{s} \_$liquid(round $\left(\left(\mathrm{P} \_\right.\right.$in-P_vapor $\left.\left.\left.(1)\right) / \mathrm{res}_{-} \mathrm{P}\right)+1\right) ; \% \mathrm{BTU} / \mathrm{lbm}$

$\mathrm{s} \_\mathrm{g}=\mathrm{s} \_$vapor(round $\left(\left(\mathrm{P} \_\right.\right.$in-P_vapor(1))/res_P $\left.)+1\right) ; \% \mathrm{BTU} / \mathrm{lbm}$

c_l = c_liquid $\left(\operatorname{round}\left(\left(\mathrm{P} \_\right.\right.\right.$in-P_vapor $\left.(1)\right) /$ res_P $\left.)+1\right) ; \% B T U / 1 b m$

c_g = c_vapor $\left(\operatorname{round}\left(\left(\mathrm{P} \_\right.\right.\right.$in-P_vapor(1))/res_P $\left.)+1\right) ; \%$ BTU $/ 1 b m$

$\mathrm{h}(1)=\mathrm{h} \_\mathrm{i} ; \% \mathrm{BTU} / \mathrm{lbm}$

rho_1 = rho_liquid $\left(\right.$ round $\left(\left(\mathrm{P} \_\right.\right.$in-P_vapor(1))/res_P $\left.)+1\right) ; \% 1 b m / \mathrm{ft} 3$

rho_g = rho_vapor(round $\left(\left(\mathrm{P} \_\right.\right.$in-P_vapor $\left.(1)\right) /$ res_P $\left.)+1\right) ; \% 1 b m / f t 3$

$\mathrm{x}(1)=\left(\mathrm{h}(1)-\mathrm{h} \_\mathrm{l}(1)\right) /\left(\mathrm{h} \_\mathrm{g}(1)-\mathrm{h} \_\mathrm{l}(1)\right)$;

$\operatorname{rho}(1)=((1-x(1)) / \text { rho_1 }(1)+x(1) / \text { rho_g }(1))^{\wedge}-1 ; \%$ lbm/ft3

$\% \%$ Revellin Thome Dryout Model

G_SI $=\mathrm{G}^{*} 4.882 ; \% \mathrm{~kg} / \mathrm{m}^{\wedge} 2 * \mathrm{sec}$

g_SI $=9.81 ; \% \mathrm{~m} / \mathrm{s}^{\wedge} 2$

Psat $=2.55^{*} 10^{\wedge} 6 ; \% \mathrm{~Pa}$

rho_L $=953.5 ; \% \mathrm{~kg} / \mathrm{m}^{\wedge} 3$

rho_v $=63.9 ; \% \mathrm{~kg} / \mathrm{m}^{\wedge} 3$

Dh_SI $=2 * .001645 ; \% \mathrm{~m}$

sigma $=0.0067578 ; \% \mathrm{~N} / \mathrm{m}$

h_lv $=(4.066-1.443) * 10^{\wedge} 5$;

Length $=0.06 ; \% \mathrm{~m}$

q_SI $=q^{*} 1.055^{*} 10^{\wedge} 3 /\left(\right.$ A_cold $\left.* 6.452^{*} 10^{\wedge}-4\right) /\left(1-z \_\exp (\right.$ first $\left.) / L \_a n n\right) ; \% \mathrm{~W} / \mathrm{m}^{\wedge} 2$

muv $=1.388 * 10^{\wedge}-5 ; \% \mathrm{~kg} / \mathrm{m} * \mathrm{sec}$

$\mathrm{mul}=1.176^{*} 10^{\wedge}-4 ; \% \mathrm{~kg} / \mathrm{m} * \mathrm{sec}$

$\mathrm{x} \_$in $=\mathrm{x}(1)$;

\%CALCULATE ANNULUS PROPERTIES AND SET BOUNDARY CONDITIONS

$\mathrm{R}=\mathrm{Dh} / 2$;

$\mathrm{P}=2 *$ pi $* \mathrm{R} ; \% \mathrm{~m}$

$\mathrm{L}=.5 ; \% \mathrm{~m}$ 


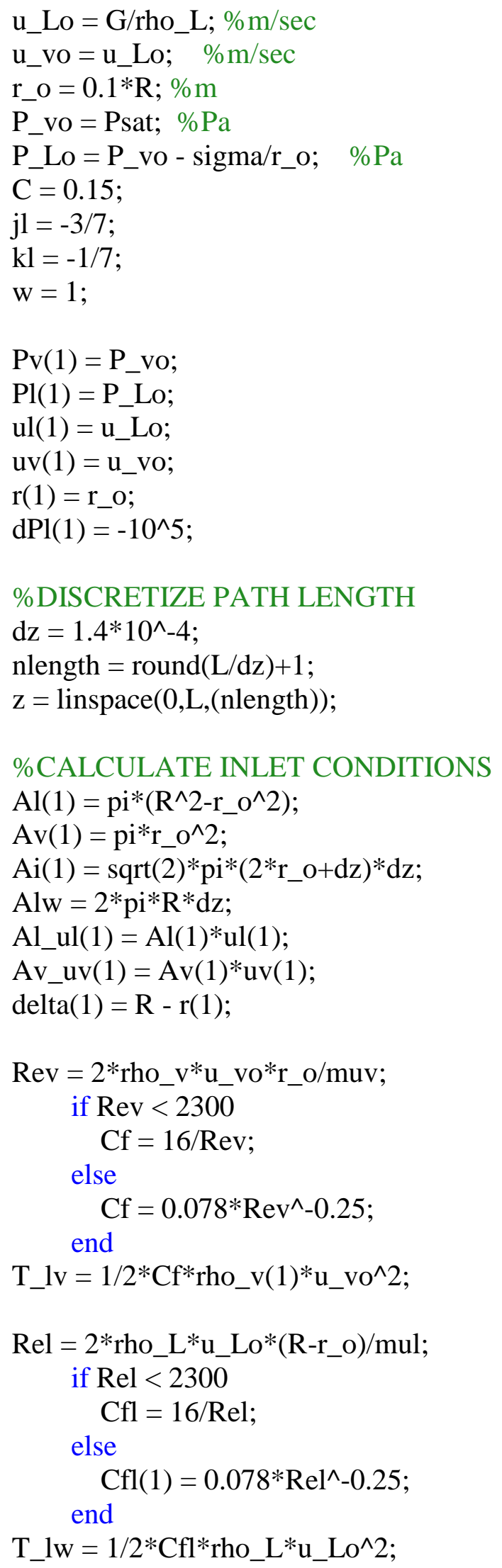




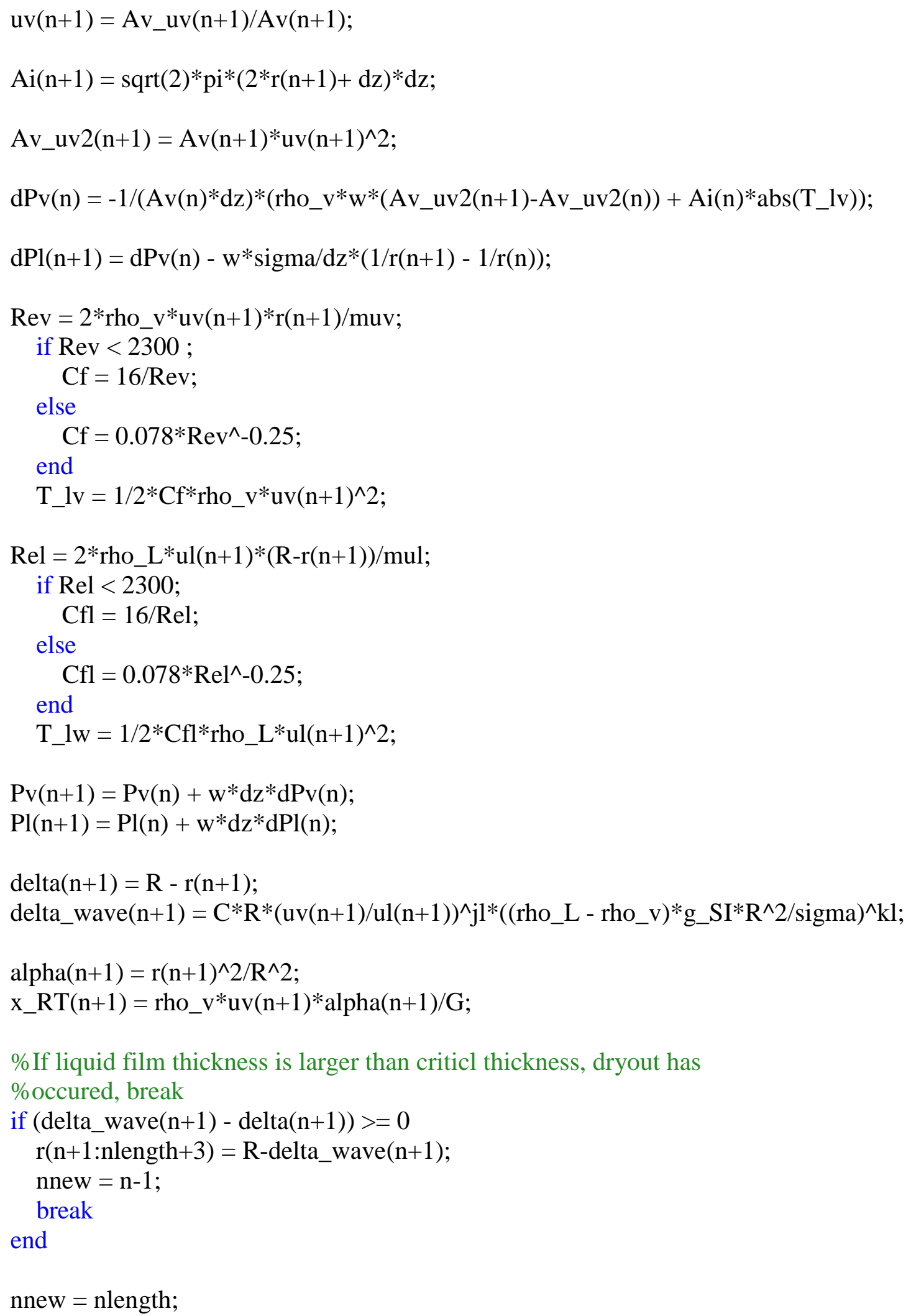




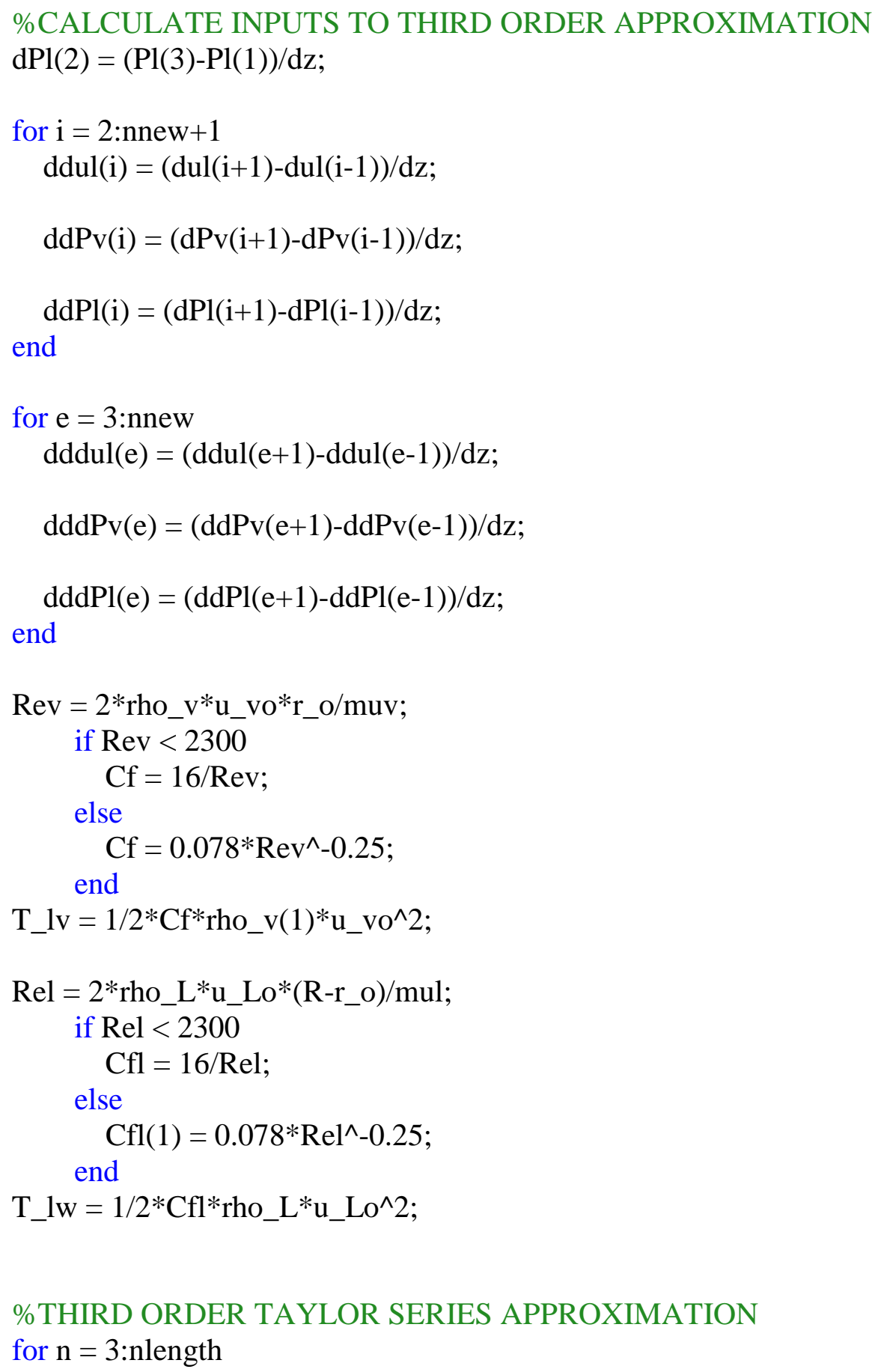




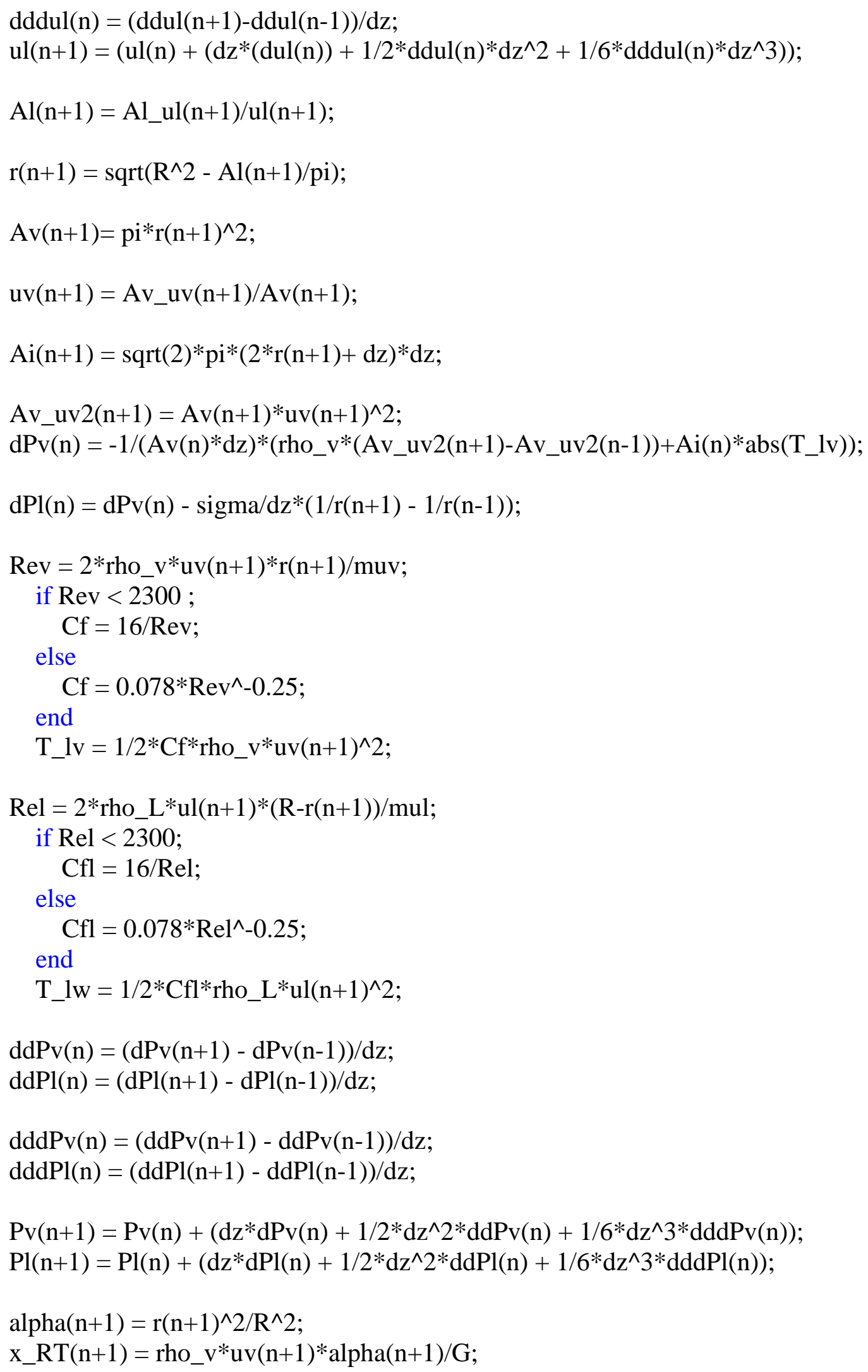




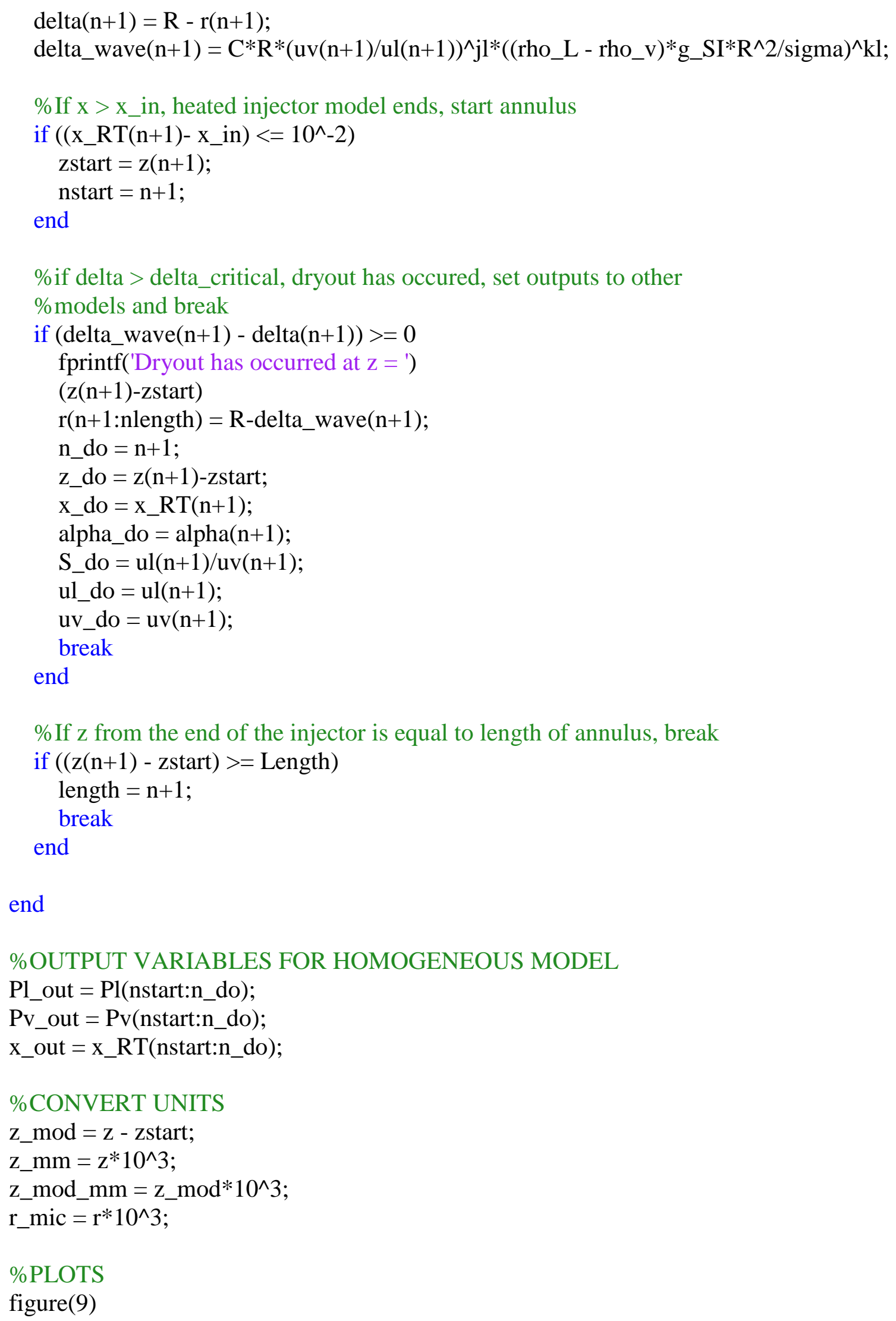


plot(z_mod_mm(1:n_do), r_mic(1:n_do), z_mod_mm(1:n_do), -r_mic(1:n_do), linspace $(0,0)$, linspace $\left(-\mathrm{R}^{*} 10^{\wedge} 3, \mathrm{R} * 10^{\wedge} 3\right)$, 'k-')

xlabel('Heated Length $\left.(\mathrm{mm})^{\prime}\right)$

ylabel('Radius (mm)')

figure(10)

plot $\left(\mathrm{z} \_\right.$mod_mm $\left(1: \mathrm{n} \_d o\right), \mathrm{Pl}\left(1: \mathrm{n} \_d o\right)^{*} 10^{\wedge}-6, \mathrm{z} \_$mod_mm $\left(1: \mathrm{n} \_\right.$do $), \operatorname{Pv}\left(1: \mathrm{n} \_d o\right)^{*} 10^{\wedge}$ 6, linspace $\left.(0,0), \operatorname{linspace}\left(\min (\mathrm{Pl}) * 10^{\wedge}-6, \max (\mathrm{Pl})^{*} 10^{\wedge}-6\right), \mathrm{k}^{--}\right)$

xlabel('Heated Length $(\mathrm{mm})$ ')

ylabel('Pressure (MPa)')

legend('Liquid', 'Vapor')

figure(11)

plot(z_mod_mm(1:n_do $), u l\left(1: n \_d o\right), z \_m o d \_m m\left(1: n \_d o\right), u v\left(1: n \_d o\right)$, linspace (0,0), linspace(min(uv), $\max (u v))$, 'k--')

xlabel('Heated Length ( $\left.\mathrm{mm})^{\prime}\right)$

ylabel('Velocity $\left.(\mathrm{m} / \mathrm{s})^{\prime}\right)$

legend('Liquid', 'Vapor')

figure(12)

plot(z_mod_mm(1:n_do $), x \_R T\left(1: n \_d o\right), \operatorname{linspace}(0,0), \operatorname{linspace}\left(\min \left(\mathrm{x} \_R T\right), \max \left(\mathrm{x} \_R T\right)\right)$, 'k--')

xlabel('Heated Length $\left.(\mathrm{mm})^{\prime}\right)$

ylabel('Quality')

pause

$\% \%$ Homogeneous Flow Model

$\%$ SET UP MESH

bias $=100$;

$\mathrm{L}=2.38067 ; \%$ inches

$\mathrm{dz}=\mathrm{L} / \mathrm{bias} ; \%$ inches

$\mathrm{dq}=\mathrm{q} * \mathrm{dz} / \mathrm{L} ; \% \mathrm{BTU} / \mathrm{sec}$

$\mathrm{z}=$ linspace $\left(\mathrm{z} \_\exp (\right.$ first $), \mathrm{L}$, bias+1); \%inches

\%INITIALIZE INLET VARIABLES TO INLET CONDITIONS

$\mathrm{h} \_$old $=\mathrm{h}(1)^{*}$ ones $(1$, bias +1$) ; \%$ BTU/lbm

$\mathrm{u}(1)=\mathrm{G} / \mathrm{rho}(1) ; \% \mathrm{ft} / \mathrm{sec}$

$\mathrm{u}(2)=\mathrm{u}(1) ; \% \mathrm{ft} / \mathrm{sec}$

$\mathrm{P}(1)=\mathrm{P} \_$in; \%psia

$\mathrm{T}(1)=\mathrm{T} \_\operatorname{vapor}\left(\operatorname{round}\left(\left(\mathrm{P} \_\right.\right.\right.$in $-\mathrm{P} \_$vapor(1))/res_P+1)$) ; \% \operatorname{degF}$

$\mathrm{v}(1)=1 / \mathrm{rho}(1) ; \% \mathrm{ft} \wedge 3 / \mathrm{lbm}$

alpha $(1)=$ rho_l $(1) * x(1) /($ rho_l $1(1) * x(1)+$ rho_g $(1) *(1-x(1)))$;

loc_T $(1)=\left(\mathrm{T}(1)-\mathrm{T} \_p r o p(1)\right) /$ res_T_prop;

mu_l$(1)=$ mu_liquid $\left(\right.$ round $\left.\left(l o c \_T(1)\right)\right)$;

mu_g $(1)=$ mu_gas $\left(\operatorname{round}\left(\operatorname{loc} \_T(1)\right)\right)$; 


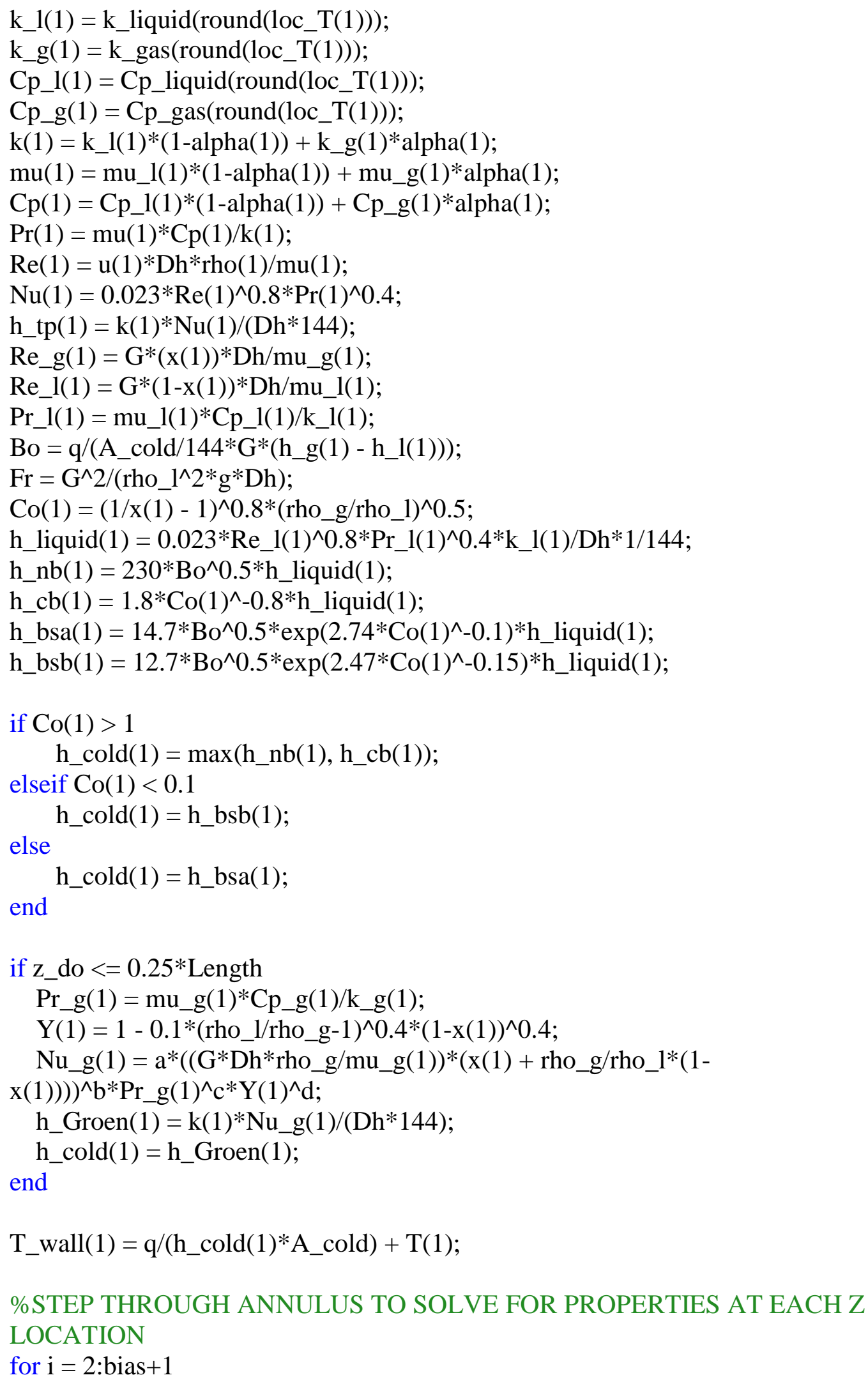




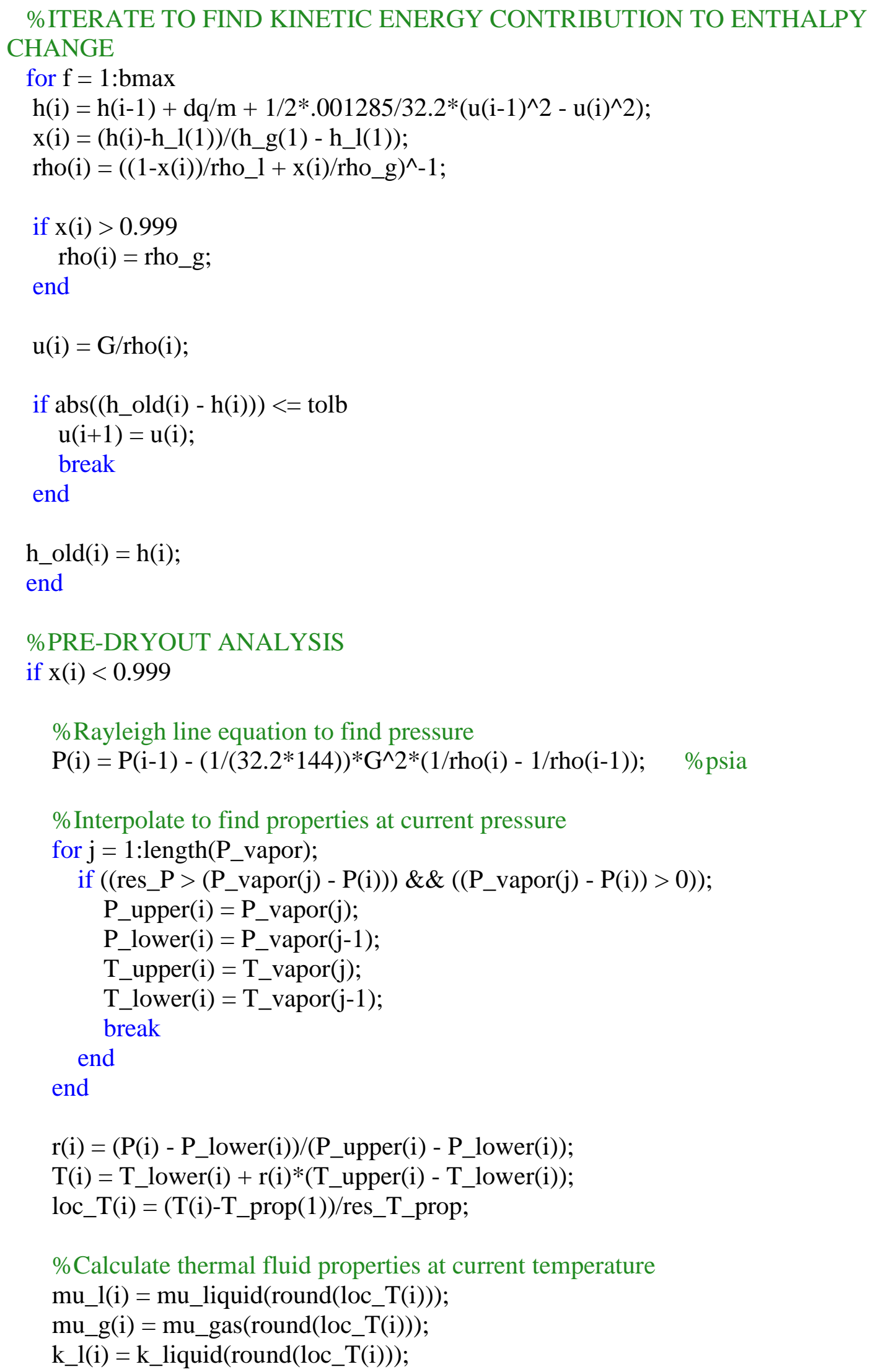


k_g(i) $=$ k_gas $($ round $($ loc_T(i) ));

Cp_l(i) $=$ Cp_liquid $\left(\right.$ round $\left.\left(l o c \_T(i)\right)\right)$;

Cp_g(i) $=$ Cp_gas $($ round $($ loc_T(i) $))$;

$\%$ Calculate flow characteristics

alpha(i) $=$ rho_l*x(i)/(rho_l*x(i) + rho_g*(1-x(i)));

$\mathrm{v}(\mathrm{i})=1 / \mathrm{rho}(\mathrm{i})$;

rho(i) $=$ rho_ $1 *(1-$ alpha(i) $)+$ rho_g*alpha $(i)$;

$\mathrm{k}(\mathrm{i})=\mathrm{k} \_\mathrm{l}(\mathrm{i}) *(1-\mathrm{alph} a(\mathrm{i}))+\mathrm{k} \_\mathrm{g}(\mathrm{i}) *$ alpha(i);

rho2(i) $=\left((1-\mathrm{x}(\mathrm{i})) / \text { rho_l } 1+\mathrm{x}(\mathrm{i}) / \mathrm{rho} \_\mathrm{g}\right)^{\wedge}-1$;

$\mathrm{mu}(\mathrm{i})=$ mu_l$(\mathrm{i}) *(1-$ alpha(i) $)+$ mu_g(i)*alpha(i);

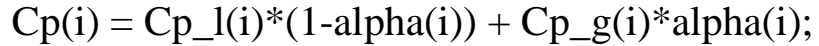

$\operatorname{Pr}(\mathrm{i})=\mathrm{mu}(\mathrm{i}) * \mathrm{Cp}(\mathrm{i}) / \mathrm{k}(\mathrm{i})$;

$\operatorname{Re}(\mathrm{i})=\mathrm{u}(\mathrm{i}) * \mathrm{Dh} * \mathrm{rho}(\mathrm{i}) / \mathrm{mu}(\mathrm{i})$;

$\mathrm{Nu}(\mathrm{i})=0.023 * \operatorname{Re}(\mathrm{i})^{\wedge} 0.8 * \operatorname{Pr}(\mathrm{i})^{\wedge} 0.4$;

$\mathrm{h} \_\mathrm{tp}(\mathrm{i})=(\mathrm{k}(\mathrm{i}) * \mathrm{Nu}(\mathrm{i}) / \mathrm{Dh}) / 144$;

$\operatorname{Re} \_\mathrm{g}(\mathrm{i})=\mathrm{G} *(\mathrm{x}(\mathrm{i}))^{*} \mathrm{Dh} / \mathrm{mu} \_\mathrm{g}(\mathrm{i})$;

Pr_g(i) $=$ mu_g(i)*Cp_g(i)/k_g(i);

Re_l(i) $=G^{*}(1-x(\mathrm{i})) * \mathrm{Dh} / \mathrm{mu} \_$l(i);

Pr_l(i) = mu_l(i)*Cp_l(i)/k_l(i);

if $\left(\mathrm{x}(\mathrm{i})-\mathrm{x} \_\mathrm{do}\right)<0 \& \&\left(\mathrm{z} \_\mathrm{do} / \mathrm{L}\right)>0.5 \%$ nucleate boiling regime (pre-dryout heat transfer coefficient)

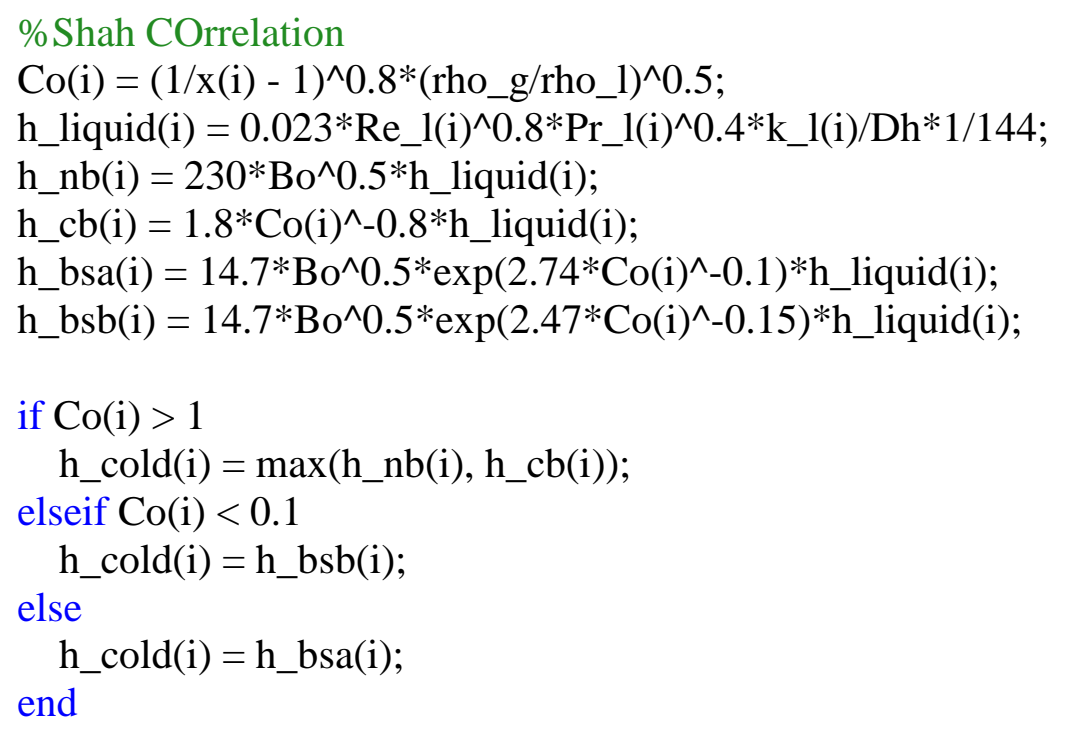

else \%mist-flow regime (post-dryout heat transfer coefficient)

$\%$ Groeneveld correlation

$\mathrm{Y}(\mathrm{i})=1-0.1^{*}(\text { rho_l} / \text { rho_g- } 1)^{\wedge} 0.4 *(1-\mathrm{x}(\mathrm{i}))^{\wedge} 0.4$;

$\mathrm{Nu} \_\mathrm{g}(\mathrm{i})=\mathrm{a}^{*}\left(\left(\mathrm{G}^{*} \mathrm{Dh} * \text { rho_g} / \mathrm{mu} \mathrm{g}(\mathrm{i})\right)^{*}(\mathrm{x}(\mathrm{i})+\right.$ rho_g$/$ rho_l*(1$\mathrm{x}(\mathrm{i}))))^{\wedge} \mathrm{b} * \operatorname{Pr} \_\mathrm{g}(\mathrm{i})^{\wedge} \mathrm{c}^{*} \mathrm{Y}(\mathrm{i})^{\wedge} \mathrm{d}$; 


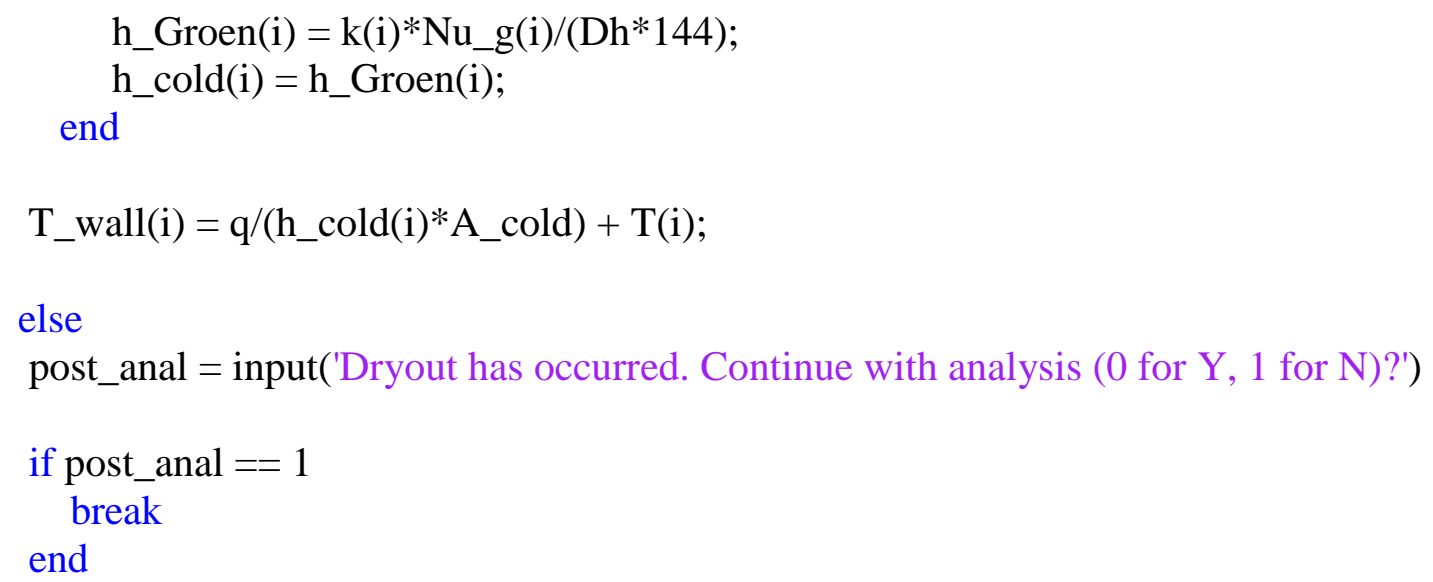

$\mathrm{T}(\mathrm{i})=\operatorname{input}\left(\left[{ }^{\prime} W\right.\right.$ hat is the temperature at ' num2str(P(i)) ' psi and ' num2str(h(i)) ' BTU/lbm ?']);

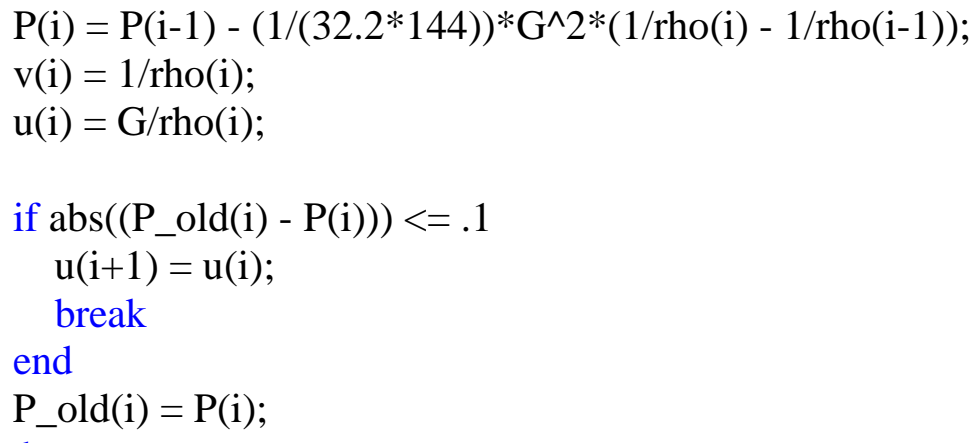

$\mathrm{Cp}(\mathrm{i})=\operatorname{input}\left(\left[{ }^{\prime} W\right.\right.$ hat is the specific heat at constant pressure Cp at ' num2str(P(i)) ' psi and ' num2str(T(i)) ' R ?']);

$\mathrm{Cv}(\mathrm{i})=\operatorname{input}\left(\left[{ }^{\prime} W h a t\right.\right.$ is the specific heat at constant volume $\mathrm{Cv}$ at ' num2str(P(i)) ' psi and ' num2str(T(i)) ' R ?']); 
?']);

$\operatorname{mu}(\mathrm{i})=\operatorname{input}\left(\left[{ }^{\prime}\right.\right.$ What is the viscosity at ' num $2 \operatorname{str}(\mathrm{P}(\mathrm{i})){ }^{\prime} \mathrm{psi}$ and ' num2str(T(i)) ' R

$\mathrm{k}(\mathrm{i})=\operatorname{input}\left(\left[{ }^{\prime}\right.\right.$ What is the thermal conductivty at ' num2str(P(i)) ' psi and '

$\operatorname{num} 2 \operatorname{str}(\mathrm{T}(\mathrm{i}))$ ' R ?'])*1.605*10^-4;

$\operatorname{Re}(\mathrm{i})=(\operatorname{rho}(\mathrm{i}) * \mathrm{u}(\mathrm{i}) * \mathrm{Dh}) / \mathrm{mu}(\mathrm{i})$;

$\operatorname{Pr}(\mathrm{i})=\mathrm{mu}(\mathrm{i}) * \mathrm{Cp}(\mathrm{i}) / \mathrm{k}(\mathrm{i})$;

h_cold $(\mathrm{i})=0.023 * \operatorname{Re}(\mathrm{i})^{\wedge} 0.8 * \operatorname{Pr}(\mathrm{i})^{\wedge} 0.4 * \mathrm{k}(\mathrm{i}) / \mathrm{Dh}^{*} 1 / 144$;

T_wall $(\mathrm{i})=\mathrm{q} /\left(\mathrm{h} \_\right.$cold $(\mathrm{i}) * A$ A_cold $)+\mathrm{T}(\mathrm{i})$;

$\operatorname{gamma}(\mathrm{i})=\mathrm{Cp}(\mathrm{i}) / \mathrm{Cv}(\mathrm{i})$;

$\mathrm{R}(\mathrm{i})=\mathrm{Cp}(\mathrm{i})-\mathrm{Cv}(\mathrm{i})$;

end

end

$\%$ PLOTS

figure(13)

$\operatorname{plot}(\mathrm{z}(1$ :length $(\mathrm{x})), \mathrm{x})$

xlabel('Distance through Annulus (in)')

ylabel('Quality')

figure(6)

$\operatorname{plot}\left(\mathrm{z}(1:\right.$ length$\left.(\mathrm{T})), \mathrm{T}, \mathrm{b}^{\prime}\right)$

$\%$ xlabel('Distance through Annulus (in)')

$\%$ ylabel('Mixture Temperature (deg F)')

legend('Experimental', 'Minimum Measurement', 'Homogeneous Model Predicted')

figure(2)

$\operatorname{plot}(\mathrm{z}(1:$ length $(\mathrm{P})), \mathrm{P}, \mathrm{b}$ ')

$\%$ xlabel('Distance through Annulus (in)')

$\%$ ylabel('Mixture Pressure (psia)')

legend('Experimental', 'Homogeneous Model Predicted')

figure(14)

plot(z(1:length(h_cold)), h_cold, 'b', z_exp, h_exp, 'kd')

xlabel('Distance through Annulus (in)')

ylabel('Coolant Heat Transfer Coefficient (BTU/in^2secR)')

legend('Experimental', 'Groeneveld Correlation')

figure(4)

$\operatorname{plot}\left(z(1:\right.$ length(T_wall) $), T_{-}$wall, 'b')

$\%$ xlabel('Distance through Annulus (in)')

\% ylabel('Copper Wall Temperature (deg F)')

legend('Experimental', 'Homogeneous Model Predicted') 